\title{
SYMPOSIUM SUMMARIES
}

\section{S1.1 \\ CF IN MICE - DISSECTING CF PATHOGENESIS THROUGH UNDERSTANDING TISSUE SPECIFIC CFTR REGULATION}

\author{
Mitchell L. Drumm, Ph.D., Tracey L. Bonfield, Ph.D. and Craig A. Hodges, Ph.D. \\ Depts. of Pediatrics \& Genetics, Case Western Reserve Univ., Cleveland, OH, USA
}

$\mathrm{CF}$ is unquestionably a systemic disorder, affecting every organ system of the body to some degree. What is not clear is how loss of CFTR gives rise to the myriad of physiologic effects characteristic of CF. For the past several decades, most of the focus has been on epithelial cells and epithelia, as they are clearly affected by the loss of CFTR and provide an explanation for elevated sweat electrolytes, airway mucous rheology and some gastrointestinal manifestations. However, CFTR mRNA is expressed in most cell types of the body and channel activity has been reported in many as well (lymphocytes, muscle, neurons, for example), but its role in most non-epithelial cells is not clear. Even less clear is the effect of not having CFTR in these non-epithelial cell types.

We have begun to address this concept in an animal model, the mouse, in which the murine Cftr gene has been modified with loxP sites to allow conditional inactivation of Cftr (1). This allele has been used to examine the effect of Cftr absence from lymphocytes and showed that mice lacking Cftr in CD3+ lymphocytes have an exuberant Th-2-biased response to Aspergillus, resembling the response of CF patients and indicating that this is a function of the $\mathrm{CF}$ immune system rather than the $\mathrm{CF}$ epithelium (2). More recently, we have inactivated $\mathrm{Cftr}$ in myeloid cells and challenged these mice with an inoculum of $P$. aeruginosa and compared the effects to mice that had received bone marrow transplants. All mice mounted an inflammatory response, but whereas control mice resolved the response within a few days, CF mice, non-CF mice with myeloid Cftr inactivation and non-CF mice receiving $\mathrm{CF}$ bone marrow all experienced prolonged inflammatory indices ( 2 weeks). These results also point to dysregulated inflammatory responses by the CF immune system.

Conditional inactivation of $\mathrm{Cftr}$ provides insight into disease pathogenesis, but another important concept is whether correcting for the loss of Cftr can alter CF pathophysiology. To begin to address this concept, a second conditional allele was generated, but in this case a nonfunctional form of Cftr was generated with loxP sites but constructed so that it could be conditionally activated. Both alleles were used to examine the effects of manipulating Cftr function in gastrointestinal epithelium. Inactivating $\mathrm{Cftr}$ in gut epithelium resulted in intestinal obstruction, but did not impair growth. Restricting Cftr function to the gut epithelium completely prevented obstruction, but these mice were indistinguishable from CF mice in terms of growth characteristics, length and weight (3).

As the organ systems affected by CF do not function in isolation, it is important to know how loss of CFTR in one cell type, tissue or organ affects the function of other cells, tissues or organs, and these novel mouse models provide insight into these interactions.

References: 1. Hodges CA, Cotton CU, Palmert MR, et al. Generation of a conditional null allele for $\mathrm{Cftr}$ in mice. Genesis. 2008;46:546-52.

2. Mueller C, Braag SA, Keeler A, et al. Lack of Cftr in CD3+ lymphocytes leads to aberrant cytokine secretion and hyper-inflammatory adaptive immune responses. Am J Respir Cell Mol Biol. 2011;44:922-9.

3. Hodges CA, Grady BR, Mishra K, et al. Cystic fibrosis growth retardation is not correlated with loss of Cftr in the intestinal epithelium. Am J Physiol Gastrointest Liver Physiol. 2011;Jun 9. PMID:21659619.

\section{S1.2 \\ CF IN PIGS - MODELING CF LUNG DISEASE}

\author{
David K. Meyerholz, D.V.M., Ph.D. \\ Pathology, Univ. of Iowa, Iowa City, IA, USA
}

Cystic fibrosis (CF) is caused by mutations in the gene encoding the $\mathrm{CF}$ transmembrane conductance regulator (CFTR). CF is characterized by clinical disease in many organs including the intestines (meconium ileus), pancreas, liver/gallbladder, skin, nasosinus, vas deferens and lung. However, the understanding of how CFTR loss causes disease phenotypes in these diverse organs is limited. Because lung disease is the principal cause of mor- tality in $\mathrm{CF}$, it is an important area of investigational study. CF mouse models were developed nearly twenty years ago and from these, much has been learned regarding CFTR and CF disease. Recently, the advent of pig and ferret models has provided new perspectives for understanding CF disease and pathogenesis. In pigs, both CFTR-Null and CFTR- $\Delta$ F508 models were born with meconium ileus, pancreatic destruction, parameters of 
focal biliary cirrhosis, and segmental atresia of the vas deferens; however, at birth, the lung lacked inflammation or airway obstruction typical of advanced CF lung disease (1-3). To determine if CF pigs would develop lung disease, we surgically corrected the meconium ileus of neonatal pigs and observed their postnatal growth for clinical evidence of lung disease. In the weeks and months after surgery, serial CT scans, bronchoalveolar lavages, cultures, and pathologic examinations revealed that $\mathrm{CF}$ pigs developed lung disease characterized by a heterogenous, intermittent to progressive process causing mucopurulent airway obstruction with air trapping, atelectasis and infection by a variety of bacterial pathogens (3-4). To better understand the mechanisms responsible for disease, we examined electrolyte transport in newborn CF airways; we found a loss of chloride and bicarbonate transport with no increase in sodium absorption (5). We challenged newborn CF pigs with $S$. aureus (clinical isolate from a $\mathrm{CF}$ pig) and found that within hours of inoculation, CF pigs had impaired eradication of bacteria compared to their wild-type littermates. These data suggest that lack of CFTR caused a host defense defect in eradicating bacteria before the onset of airway inflammation (4). We also morphometrically examined trachea and large airways to see if loss of CFTR caused microanatomic changes. CF trachea and primary bronchi had reduced caliber and circularity with hypoplastic submucosal glands, accentuated smooth muscle bundles and discontinuous cartilage along the anterior surface. We found no change in MUC5AC positive goblet cells, cellular height or ciliary height of surface epithelium. To see if the tracheal phenotype translated to humans, we retrospectively examined pediatric CT scans and published trachea data. We found that infants with CF had detectably reduced tracheal circularity and caliber as predicted by the CF pig model (6). We studied smooth muscle as a possible contributor to this phenotype because of its altered morphology at birth and because altered pathophysiology of airway smooth muscle might explain some of the observed anatomic changes including reduced caliber, reduced circulatory and discontinuous cartilage. Ongoing studies suggest a potential role of CFTR in airway smooth muscle function. We are also studying neonatal and fetal CF pig lung using CT imaging, morphometric analysis and gene expression to assess genotypic differences in airway and parenchymal structure and development. In summary, CF pigs spontaneously develop lung disease like that in humans. This model has helped us address questions regarding $\mathrm{CF}$ pathophysiology, and it is helping us identify novel features of the CF phenotype in humans, expanding our clinical understanding of the disease.

References: 1. Rogers CS, Stoltz DA, Meyerholz DK, et al. Disruption of the CFTR gene produces a model of cystic fibrosis in newborn pigs. Science. 2008;321:1837-41.

2. Meyerholz DK, Stoltz DA, Pezzulo AA, et al. Pathology of gastrointestinal organs in a porcine model of cystic fibrosis. Am J Pathol. 2010;176:1377-89.

3. Ostedgaard LS, Meyerholz DK, Chen JH, et al. The $\triangle$ F508 mutation causes CFTR misprocessing and cystic fibrosis-like disease in pigs. Sci Transl Med. 2011;3:74ra24.

4. Stoltz DA, Meyerholz DK, Pezzulo AA, et al. Cystic fibrosis pigs develop lung disease and exhibit defective bacterial eradication at birth. Sci Transl Med. 2010;2:29ra31.

5. Chen JH, Stoltz DA, Karp PH, et al. Loss of anion transport without increased sodium absorption characterizes newborn porcine cystic fibrosis airway epithelia. Cell. 2010;143:911-23.

6. Meyerholz DK, Stoltz DA, Namati E, et al. Loss of cystic fibrosis transmembrane conductance regulator function produces abnormalities in tracheal development in neonatal pigs and young children. Am J Respir Crit Care Med. 2010;182:1251-61.

\title{
S1.3 \\ CF IN FERRETS - MODELING CF DIABETES AND LUNG DISEASE
}

\author{
John F. Engelhardt, Ph.D. \\ Dept. of Anatomy \& Cell Biology, Univ. of Iowa, Iowa City, IA, USA
}

Animal modeling of cystic fibrosis has been challenging, with species- and strain-specific differences in organ biology and CFTR function influencing the emergence of disease pathology. We have developed a CFTR-deficient ferret model (1) that develops many of the pathologies observed in humans with $\mathrm{CF}$, including defective airway chloride transport and submucosal gland fluid secretion, variably penetrant meconium ileus, pancreatic, liver and vas deferens disease, and most importantly lung infections during the neonatal period (2). Newborn CFTR-knockout ferrets fail to thrive and succumb to lung infection if untreated shortly after birth. This phe- notype can be partially overcome by antibiotic therapy, supplementation with elemental diet, and drug therapy to raise gastrointestinal $\mathrm{pH}$ and normalize liver function tests. Using these rearing methods, CF ferrets can be reared to adulthood. In contrast to neonates, adult $\mathrm{CF}$ ferrets contract a more slowly progressive lung disease characterized by excessive mucus production, plugging of the airways, and lethal bacterial infections.

To determine whether early lung infections during the first weeks of life observed in CFTR-knockout ferrets were secondary to impaired nutritional status, we performed controlled bacterial challenge experiments in $\mathrm{KO}$ 
and WT newborns within 6-12 hrs after birth. At this time, total weight and body mass index were similar between the two genotypes. Controlled injection of antibiotic resistant PAO1 into the trachea of newborn $\mathrm{KO}$ and WT animals demonstrated two orders of magnitude difference in bacterial killing over a $6 \mathrm{hr}$ period. Interestingly, newborn (0-3 day old) WT and KO animals demonstrated no significant differences in mucociliary clearance, suggesting that defects in bacterial eradication were not due to impaired clearance during the newborn interval. However, mucociliary clearance rapidly became impaired within CF animals by 1-2 weeks of life.

With advances in medical treatments for CF lung disease, the incidence of CF-related diabetes (CFRD) is climbing-now $\sim 50 \%$ of CF patients develop CFRD by the age of 30 years $(3,4)$. Although CFRD is only infrequently seen in infants, $20 \%$ of adolescents are affected (5). CFRD is now the main secondary complication in $\mathrm{CF}$, and responsible for a 6-fold increase in morbidity and mortality (3). The pleiotropic nature of CFRD, which does not conform to either type 1 or type 2 diabetes, has made dissection of its etiology and impact on CF lung disease difficult to understand. Declining clinical status and lung function often occurs years before CFRD is diagnosed, and it is uncertain if the pre-diabetic state influences the onset of more severe lung disease or if more severe lung disease influences emergence of CFRD.

We have begun to utilize the CFTR-knockout ferret model to address the pathophysiology of CFRD. Although histopathology is mild in the CF ferret pancreas at birth, pancreatic disease in CFTR-knockout ferrets is rapidly progressive and leads to destruction of both the exocrine and endocrine pancreas during the first month of life. Such pathology is accompanied by hyper- glycaemia, glycosuria, and fibrosis of the pancreas. To evaluate whether defects in the endocrine pancreas occur prior to the emergence of pancreatic fibrosis and destruction of the exocrine pancreas, we evaluated endocrine pancreas structure and function in newborn CFTRknockout ferrets. Newborn CFTR-knockout ferrets demonstrated no significant changes in the percentage of parenchymal insulin and glucagon positivity in the pancreas. Despite these findings, subtle abnormalities in glycemic regulation were observed. For example, newborn CF ferrets demonstrate abnormal glucose tolerance tests despite the minimal histopathology in the pancreas at birth. CF kits also demonstrate impaired acute insulin secretion following L-arginine or glucose challenge and an exaggerated second phase insulin response to glucose. These findings have begun to implicate CFTR functions in the endocrine pancreas that may contribute early in life to the metabolic and lung pathology in cystic fibrosis.

References: 1. Sun X, Yan Z, Yi Y, et al. Adeno-associated virus-targeted disruption of the CFTR gene in cloned ferrets. J Clin Invest. 2008;118:1578-83.

2. Sun X, Sui H, Fisher JT, et al. Disease phenotype of a ferret CFTR-knockout model of cystic fibrosis. J Clin Invest. 2010;120:3149-60.

3. O’Riordan SM, Dattani MT, Hindmarsh PC. Cystic fibrosis-related diabetes in childhood. Horm Res Paediatr. 2010;73:15-24.

4. Moran A, Becker D, Casella SJ, et al. Epidemiology, pathophysiology, and prognostic implications of cystic fibrosis-related diabetes: a technical review. Diabetes Care. 2010;33:2677-83.

5. Moran A, Dunitz J, Nathan B, et al. Cystic fibrosisrelated diabetes: current trends in prevalence, incidence, and mortality. Diabetes Care. 2009;32:1626-31.

\title{
S1.4 "MAN IS THE ONLY ANIMAL THAT BLUSHES — OR NEEDS TO"
}

\author{
Paul M. Quinton, Ph.D. \\ 1. Pediatrics, UCSD School of Medicine \& Rady Children's Hospital, La Jolla/San Diego, CA, USA; 2. Biomedical \\ Sciences, UC Riverside, Riverside, CA, USA
}

It is embarrassing that after nearly three decades of defining electrolyte disorders in cystic fibrosis $(\mathrm{CF})$, we do not yet have a unifying cause-and-effect mechanism to explain why a basic defect in anion transport causes angry mucus in CF. Understanding what make mucus hostile enough to kill should be critical to design strategies for effective therapy.

To date the most widely held concept for explaining the abnormally "thick" CF mucus holds that $\mathrm{Na}^{+}$dependent fluid absorption via $\mathrm{ENaC}$ is exuberant and excessive in CF, causing "dehydrated" mucus. Since thick mucus, or mucoviscidosis, underlies the pathology of all anatomically distressed tissues in $\mathrm{CF}$, the same basis or phe- nomenon that causes abnormal mucus should occur in each affected organ. Yet, four of the major organs that are affected by thickened mucus in CF cannot "dehydrate" mucus via hyperactive $\mathrm{ENaC}$ because they lack this cation channel for absorbing $\mathrm{Na}^{+}$and fluid. Consequently, another mechanism common to mucus formation and dependent on CFTR should be present in those organs. An inspection of the organs affected in $\mathrm{CF}$ suggests that depressed $\mathrm{HCO}_{3}{ }^{-}$transport is a common defect in these systems.

This fact raises the question of whether altering $\mathrm{HCO}_{3}^{-}$transport alters mucus. Briefly, we and others have recently observed that disruption of $\mathrm{HCO}_{3}^{-}$secre- 
tion concurrent with mucus secretion causes several alterations that appear consistent with mucus abnormalities presumed to occur in $\mathrm{CF}$, namely: 1) mucus discharge is impeded; 2) mucus swelling is reduced; 3) mucus diffusivity is decreased (viscosity is increased); 4) mucus adhesiveness appears increased; and 5) stimulated mucocilliary transport is diminished.

In this presentation, we will try to: 1) compare some of the more outstanding disturbances in mucus secretion and clearance in humans and animal models; 2) defend the notion that poor $\mathrm{HCO}_{3}^{-}$transport is common to $\mathrm{CF}$ affected tissues; and 3) explain at least to a limited extent in terms of electrostatics why $\mathrm{HCO}_{3}{ }^{-}$is critical for normal mucin release and formation.

We will consider the possible importance of these observations to adapt strategies for therapy with $\mathrm{HCO}_{3}^{-}$. We will discuss these findings and their potential relevance to approaches by which mucocilliary clearance might be improved in airways of human animals - no joke.

Acknowledgments: The authors' work is supported by MCCC-Cystic Fibrosis Foundation; NIH-RO1 HL084042; CFRI, and Nancy Olmsted Endowment. Special thanks to Mr. S. Clemens for assistance with the title.

\title{
S2.1 \\ AN OVERVIEW OF VITAMIN D METABOLISM
}

\author{
Vin Tangpricha, M.D., Ph.D. \\ 1. Endocrinology/Medicine, Emory Univ. School of Medicine, Atlanta, GA, USA; 2. Nutrition and Health Sciences \\ Program, Emory Laney Graduate School, Atlanta, GA, USA
}

Vitamin D is an important secosteroid hormone for the maintenance of normal calcium homeostasis for optimal skeletal mineralization. Vitamin D can be produced in the skin or obtained from the diet from a limited number of foods. After entering the circulation, vitamin D is converted to 25-hydroxyvitamin $\mathrm{D}(25(\mathrm{OH}) \mathrm{D})$ by enzymes in the liver and then to 1,25-dihydroxyvitamin $\mathrm{D}(1,25(\mathrm{OH}) 2 \mathrm{D})$, the more active form of vitamin $\mathrm{D}$, by enzymes in the kidney (1). Other tissues in the body have the ability to convert $25(\mathrm{OH}) \mathrm{D}$ to $1,25(\mathrm{OH}) 2 \mathrm{D}$, which may act as an autocrine and/or paracrine hormone to regulate other extra-skeletal processes (2).

Patients with cystic fibrosis $(\mathrm{CF})$ have been found to have sub-optimal vitamin $D$ status by several recent published reports $(3,4)$. Potential causes of vitamin D deficiency in $\mathrm{CF}$ include fat malabsorption due to pancreatic insufficiency (5), inadequate sunlight exposure (6), suboptimal supplementation and/or intake of vitamin D containing foods, diminished vitamin D stores due to decreased adiposity, and decreased circulating half-life of $25(\mathrm{OH}) \mathrm{D}$ due to alterations in vitamin $\mathrm{D}$ binding protein (4).

Randomized controlled trials have demonstrated efficacy of vitamin D therapy in the prevention of osteoporotic fractures in non-CF patients when adequate levels of serum $25(\mathrm{OH}) \mathrm{D}$ are obtained and when adherence to vitamin $\mathrm{D}$ is high (1). Recent vitamin D guidelines from the Institute of Medicine have focused on optimizing bone health for the general population (7). More recent guidelines developed in 2011 co-sponsored by the Endocrine Society, Canadian Society of Endocrinology and Metabolism and the National Osteoporosis Foundation call for screening of vitamin D deficiency in patients with $\mathrm{CF}$ and recommend much higher levels of serum $25(\mathrm{OH}) \mathrm{D}$ and higher doses of vitamin $\mathrm{D}$ in those at risk for vitamin $\mathrm{D}$ deficiency, including patients with CF (1).

Although direct randomized controlled trial evidence in the CF population is currently lacking, vitamin D deficiency is a likely major contributor to $\mathrm{CF}$ bone disease. More focus should be placed on prevention of vitamin D deficiency in the CF population given that it is well established that vitamin D deficiency is nearly universal in $\mathrm{CF}$ patients. New guidelines to be released by the $\mathrm{CF}$ Foundation will address screening, prevention and treatment of vitamin D deficiency in the CF population.

References: 1. Holick MF, Binkley NC, BischoffFerrari HA, et al. Evaluation, treatment, and prevention of vitamin D deficiency: An Endocrine Society Clinical Practice Guideline. J Clin Endocrinol Metab. 2011 Jun 6. [Epub ahead of print]

2. Kamen DL, Tangpricha V. Vitamin D and molecular actions on the immune system: modulation of innate and autoimmunity. J Mol Med. 2010;88:441-50.

3. Wolfenden LL, Judd SE, Shah R, et al. Vitamin D and bone health in adults with cystic fibrosis. Clin Endocrinol (Oxf). 2008;69:374-81.

4. Hall WB, Sparks AA, Aris RM. Vitamin d deficiency in cystic fibrosis. Int J Endocrinol. 2010;2010:218691.

5. Lark RK, Lester GE, Ontjes DA, et al. Diminished and erratic absorption of ergocalciferol in adult cystic fibrosis patients. Am J Clin Nutr. 2001;73:602-6.

6. Robberecht E, Vandewalle S. Cholecalciferol and 25-hydroxyvitamin D concentrations in adults with cystic fibrosis. Am J Clin Nutr. 2008;87:190.

7. Heaney RP, Holick MF. Why the IOM recommendations for vitamin D are deficient. J Bone Miner Res. 2011 Jan 4. [Epub ahead of print] 


\title{
S2.2 \\ A SUMMARY OF THE CYSTIC FIBROSIS VITAMIN D EVIDENCE-BASED REVIEW AND GUIDELINES
}

\author{
Karen M. Maguiness, MS, RD, CSP \\ Pediatric Pulmonology, Indiana Univ. School of Medicine, Indianapolis, IN, USA
}

Vitamin D deficiency is a concern for the general population, but particularly for individuals with cystic fibrosis, the majority of whom experience maldigestion with resultant malabsorption. Knowing how to treat and prevent vitamin $\mathrm{D}$ deficiency is of utmost importance for optimal nutritional care. For this reason, the Cystic Fibrosis Foundation formed an evidence based guidelines committee, to develop practice guidelines regarding the assessment and management of vitamin D levels in persons with cystic fibrosis (1). Using questions developed by this committee, a Cochrane systematic review was performed. Figure 1 details a summary of the search and review process.

For the assessment of vitamin D status and treatment goals, the CF Foundation recommends that all individuals with CF have serum 25-hydroxyvitamin D measured annually, preferably at the end of winter, with a goal to maintain serum 25-hydroxyvitamin D at $\geq 30 \mathrm{ng} / \mathrm{mL}$, but not to exceed $100 \mathrm{ng} / \mathrm{mL}$. For individuals with $\mathrm{CF}$ with a serum 25-hydroxyvitamin D level $<30 \mathrm{ng} / \mathrm{mL}$, there should be assessment for adherence to the prescribed regimen. The CF Foundation recommends against the use of serum 1,25-dihydroxyvitamin $\mathrm{D}$ as the measurement to assess vitamin D status and against the routine measurement of parathyroid hormone, osteocalcin, alkaline phosphatase, or other indirect markers to assess vitamin D status in all individuals with CF. The CF Foundation is not able to make a recommendation for or against having all individuals with $\mathrm{CF}$ fast prior to the measurement of serum 25-hydroxyvitamin D. All individuals with CF should have serum 25-hydroxyvitamin D levels rechecked after 3 months any time the dose of vitamin D3 has been changed. All of the above recommendations for the assessment of vitamin D status and treatment goals are based on consensus recommendations and have a low certainty of net benefit using the USPSTF grading system.

Regarding the use of vitamin D compounds and treatment strategy, the CF Foundation recommends all individuals with $\mathrm{CF}$ be treated with vitamin D3 (cholecalciferol) to achieve and maintain serum 25-hydroxyvitamin $\mathrm{D}$ levels $\geq 30 \mathrm{ng} / \mathrm{mL}$. This recommendation is made with moderate certainty and moderate benefit using the USPSTF grading system; all other recommendations in this section have a low certainty and unknown benefit. The CF Foundation is not able to make a recommendation for or against the use of an oil versus a powder-based formulation of vitamin D3 in all individuals with $\mathrm{CF}$. The CF Foundation recommends all individuals with $\mathrm{CF}$ who are prescribed vitamin D3 (in addition to their CF-specific vitamins) take once-daily vitamin D3 therapy or its weekly dose equivalent to maintain serum 25-hydroxyvitamin D levels $\geq 30 \mathrm{ng} / \mathrm{mL}$, and those individuals with refractory vitamin D deficiency be treated with calcitriol, doxercalciferol or paricalcitol only in consultation with a specialist with expertise in vitamin D therapy.

For the treatment of infants with $\mathrm{CF}$, birth to 12 months, the CF Foundation recommends an initial dose of 400-500 IU vitamin D3 per day. This dose is provided in $1 \mathrm{ml}$ of CF specific multivitamins. For infants with a serum 25-hydroxyvitamin D level $<10 \mathrm{ng} / \mathrm{mL}$, it is recommended that assessment for rickets and urgent management and treatment in consultation with a specialist with expertise in vitamin $\mathrm{D}$ therapy be completed. For infants with a serum 25-hydroxyvitamin D level $\geq 20$ but $<30 \mathrm{ng} / \mathrm{mL}$ and with confirmed adherence to the prescribed regimen, the dose of vitamin D3 may be increased to 800-1000 IU per day. For infants with a serum 25 -hydroxyvitamin $\mathrm{D}$ level $<20 \mathrm{ng} / \mathrm{mL}$ or with a persistent serum 25-hydroxyvitamin $\mathrm{D}$ level $\geq 20$ but $<$ $30 \mathrm{ng} / \mathrm{mL}$ and with confirmed adherence, the dose of vitamin D3 may be increased to a maximum of 2000 IU per day. The CF Foundation recommends all individuals with $\mathrm{CF}$, birth-12 months, who are unable to achieve a serum 25 -hydroxyvitamin D level $\geq 30 \mathrm{ng} / \mathrm{mL}$ after treatment with 2000 IU vitamin D3 per day and with confirmed adherence, be managed in consultation with a specialist with expertise in vitamin D therapy.

For treatment of children 1-10 years of age, the $\mathrm{CF}$ Foundation recommends treatment with an initial dose of 800-1000 IU vitamin D3 per day. For those with a serum 25-hydroxyvitamin D level $\geq 20$ but $<30 \mathrm{ng} / \mathrm{mL}$ and with confirmed adherence to the prescribed regimen, the dose of vitamin D3 may be increased to 1600-3000 IU per day. If serum 25-hydroxyvitamin D level $<20 \mathrm{ng} / \mathrm{mL}$ or there is a persistent serum 25-hydroxyvitamin D level $\geq 20$ but $<30 \mathrm{ng} / \mathrm{mL}$ and confirmed adherence, the dose of vitamin D3 may be increased to a maximum of 4000 IU per day. For those who are unable to achieve a serum 25 -hydroxyvitamin $\mathrm{D}$ level $\geq 30 \mathrm{ng} / \mathrm{mL}$ after treatment with 4000 IU vitamin D3 per day and with confirmed adherence, management should be done in consultation with a specialist with expertise in vitamin D therapy.

For the treatment of individuals with CF who are 10 years of age and older, the CF Foundation recommends an initial dose of 800-2000 IU vitamin D3 per day. For those with a serum 25-hydroxyvitamin D level $\geq 20$ but $<30 \mathrm{ng} / \mathrm{mL}$ and with confirmed adherence to the pre- 
scribed regimen, the dose of vitamin D3 may be increased to 1600-6000 IU per day. For those with a serum 25 -hydroxyvitamin D level $<20 \mathrm{ng} / \mathrm{mL}$ or with a persistent serum 25-hydroxyvitamin $\mathrm{D}$ level $\geq 20$ but $<$ $30 \mathrm{ng} / \mathrm{mL}$ and with confirmed adherence, the dose of vitamin D3 may be increased to a maximum of 10,000 IU per day. The CF Foundation recommends all individuals with $\mathrm{CF}$, age $>10$ years, who are unable to achieve a serum 25 -hydroxyvitamin D level $\geq 30 \mathrm{ng} / \mathrm{mL}$ after treatment with 10,000 IU vitamin D3 per day and with confirmed adherence, be managed in consultation with a specialist with expertise in vitamin D therapy. All of the above recommendations for the assessment of vitamin D status and treatment goals from birth through adulthood are based on consensus recommendations and have a low certainty of net benefit using the USPSTF grading system.
Regarding the use of UV lamps, the CF Foundation is not able to recommend for or against the use of ultraviolet lamps in the management of vitamin D deficiency in all individuals with CF. This recommendation has a low certainty and an unknown benefit, using the USPSTF grading system.

These consensus and evidence based recommendations for assessment and management of vitamin D will provide guidelines and standardized care in the treatment of individuals with CF. This evidence based review provides the framework for continued future research regarding best clinical practice in vitamin $\mathrm{D}$ management for all individuals with CF.

References: 1. Tangpricha V, Kelly A, Stephenson A, et al. An update on the screening, diagnosis, management and treatment of vitamin D deficiency in patients with cystic fibrosis: Evidence based recommendations from the Cystic Fibrosis Foundation. (in submission)

\title{
S2.3 \\ DOES TRANSPLANTATION ALTER VITAMIN D MANAGEMENT?
}

\author{
Robert Aris, M.D. \\ UNC at Chapel Hill, Chapel Hill, NC, USA
}

Probably. The complexity of this question should not be over-estimated because vitamin $\mathrm{D}$ has been shown to be an important immunoregulatory molecule vis a vis transplant rejection in addition to being involved in calcium, bone biology, and fracture prevention. Furthermore, extra-skeletal sequelae of vitamin D deficiency include insulin resistance, hypertension, and malignancy (1), all of which are major comorbidities after lung and solid organ transplantation. The following remarks will focus on the role of vitamin D and both host response to pathogen and transplant immunology. While beyond the scope of this short summary, a myriad of papers largely from the bench and pre-clinical studies, suggest that vitamin $\mathrm{D}$, mainly in the form of $1,25 \mathrm{OH}_{2} \mathrm{D}$, augments $\mathrm{CD} 4+, \mathrm{CD} 25^{\text {high }}$ regulatory $\mathrm{T}$ cells, which are able to immunomodulate allogeneic $\mathrm{T}$ cells and thus protect the lung graft from rejection (2). In addition, vitamin D regulates antigen presentation and contributes to the production of tolerogenic dendritic cells (3). Recently, two clinical studies in renal transplant recipients showed that vitamin D reduced HLA DR and costimulatory molecule expression in peripheral blood leukocytes, and expanded regulatory $\mathrm{T}$ cell populations, results which might be anticipated to have a salubrious effect on transplant rejection $(4,5)$. Taken together, these results suggest that vitamin D metabolites might protect the lung allograft by reducing overall alloreactivity (rejection). In addition through antimicrobial peptide (cathelicidin) networks and toll like receptors, vitamin D plays a crucial role in pathogen defense against bacterial and mycobacterial infections (6). In this way, vitamin D "primes" the innate immune response through macrophage and epithelial cell activation (7). Thus, in a post-transplant environment, vitamin D may have broad reaching positive effects on the health of the patient and the graft.

But what happens to serum 250HD levels after lung transplantation? First, virtually every disease that results in end-stage lung disease and the need for lung transplantation, including $\mathrm{CF}$, is associated with serious vitamin D deficiency (8). The same is true for other solid organ transplantation. Thus low 25OHD levels are the norm during advanced illness. The reported prevalence of vitamin D insufficiency after lung transplant ranges from $51 \%$ to $97 \%$ and of severe deficiency from $26 \%$ to $33 \%(8,9)$. The etiology of this problem is due the chronic disease, inadequate vitamin $\mathrm{D}$ intake and low sun exposure due to immobility. In addition, because of an increased risk of skin cancer $(7.4 \%$ incidence by year 3 and up to $80 \%$ after $20 \mathrm{yrs}$, largely linked to azathioprine use) in organ recipients, many patients dramatically limit their sun exposure (10). Fortunately newer DNA synthesis inhibitors like mycophenolate (cellcept $\left.{ }^{\circledR}\right)$ may cause less risk.

Prospective studies in lung, heart, and liver transplant recipients indicate that serum $250 \mathrm{HD}$ levels tend to gradually increase from the low levels seen before transplantation to normal levels $(9,11)$. While some of this change is probably related to the use of vitamin D supplements, it is certainly not all related because, as it pertains to CF individuals, they are already supplemented 
carefully before lung transplant. Even more importantly a number of transplant medications alter the calciumvitamin D-PTH axis. Thus, PTH, a usually reliable measure of the health of the calcium-vitamin D axis, is a poor biomarker of vitamin D deficiency after transplant because calcineurin immunosuppressants (cyclosporine and tacrolimus) reduce renal function and raise PTH levels over time. In addition calcineurin immunosuppressants raise $1,25 \mathrm{OH}_{2} \mathrm{D}$ levels and lower Vitamin $\mathrm{D}$ binding protein production, the latter of which would be expected to lower serum 25OHD levels over time (12). Furthermore, corticosteroids increase 25OHD catabolism, an effect which may explain, in part, why 25OHD levels go up over time after transplant since corticosteroids are slowed weaned down to a nadir near the 6 month anniversary (13). Lastly macrolides and azoles, used commonly to slow the advent of chronic rejection or treat fungal infections, respectively, inhibit 25OHD catabolism via the CYP3A4 enzyme and thus would contribute to higher serum 25OHD levels. Therefore vitamin $\mathrm{D}$ metabolism after transplant is very complicated. One 2-yr RCT of the efficacy of pamidronate in treating postlung transplant osteoporosis in CF patients treated with routine immunosuppressants, but not macrolides, puts things into perspective. This study demonstrated that serum 25OHD, $1,25 \mathrm{OH}_{2} \mathrm{D}$ and PTH levels went up $\sim 50 \%$ on average despite no changes in vitamin D supplementation in the patients. Since half the patients were receiving placebo, the effects were not due to bisphosphonate treatment itself (14). Interestingly other fat soluble vitamin (A and $\mathrm{E}$ ) levels go up in patients after lung transplantation, despite no difference in supplementation, attesting to the likelihood that drug-drug or drug-vitamin interactions may be taking place at the level of CYP3A4, the complexities of which have not been fully unraveled (15). Sometimes these levels go so high that cessation of supplements is a consideration.

What are the consequences of low vitamin D status after transplantation? Low vitamin D status (25OHD or $\left.1,25 \mathrm{OH}_{2} \mathrm{D}\right)$ has been linked to low femoral neck Z-scores (liver transplant), indirectly to vertebral fractures (heart transplant) and death (heart transplant) (6), while few studies linking adverse outcomes in lung transplant have been undertaken. Of course, vitamin D deficiency after organ transplant must be taken in the context of the fact that both steroids and calcineurin inhibitors are direct causes of osteoporosis and fracture, as is diabetes, a common complication after transplantation. Therefore it has been historically difficult to link a particular causal factor (eg, vitamin D insufficiency) directly to an adverse outcome because of the myriad of potentially influential and overlapping variables.

What are the best interventions for low vitamin $D$ after lung or liver transplantation? Vitamin D supplementation in various forms usually has a salubrious effect on bone endpoints, measured most commonly by bone densitometry as fracture endpoint trials are usually underpowered after transplant. Most vitamin D intervention studies have shown a slowing in bone density decline after transplant. Bisphosphonates, in this regard, are more potent drugs and are considerably better at improving bone density than vitamin D supplements alone. There is not a one-size-fits-all supplementation protocol, so supplement doses should be guided by serum 25OHD levels, similar to what is done before transplant. And realizing for all the fat soluble vitamins, some patient may require large, small or no supplements. It should also be noted that as GFR declines in lung transplant recipients over time (as a consequence of immunosuppressants), a transition from ergo- or chole-calciferol to calcitriol (Rocaltrol@) may be necessary because the kidney will no longer be able to 1-hydroxylate the precursor molecule to the most biologically active metabolite, $1,25 \mathrm{OH}_{2} \mathrm{D}$.

References: 1. Holick MF. Vitamin D deficiency. N Engl J Med. 2007;357:266-81.

2. Gorman S, Judge MA, Burchell JT, et al. 1,25Dihydroxyvitamin D3 enhances the ability of transferred CD4+ CD25+ cells to modulate $\mathrm{T}$ helper type 2-driven asthmatic responses. Immunology. 2010;130:181-92.

3. Adorini L, Penna G. Induction of tolerogenic dendritic cells by vitamin D receptor agonists. Handb Exp Pharmacol. 2009;188:251-73.

4. Ahmadpoor P, Ilkhanizadeh B, Ghasemmahdi L, et al. Effect of active vitamin D on expression of co-stimulatory molecules and HLA-DR in renal transplant recipients. Exp Clin Transplant. 2009;7:99-103.

5. Ardalan MR, Maljaei H, Shoja MM, et al. Calcitriol started in the donor, expands the population of CD4+CD25+ T cells in renal transplant recipients. Transplant Proc. 2007;39:951-3.

6. Bikle DD. Vitamin D and the immune system: role in protection against bacterial infection. Curr Opin Nephrol Hypertens. 2008; 17:348-52.

7. Liu PT, Stenger S, Li H, et al. Toll-like receptor triggering of a vitamin D-mediated human antimicrobial response. Science. 2006;311:1770-3.

8. Stein EM, Shane E. Vitamin D in organ transplantation. Osteoporos Int. 2011;7:2107-18.

9. Aris RM, Neuringer IP, Egan TM, et al. Severe osteoporosis before and after lung transplantation. Chest. 1996;109:1176-83.

10. Lee CT, $\mathrm{Ng} \mathrm{HY,} \mathrm{Lien} \mathrm{YH,} \mathrm{et} \mathrm{al.} \mathrm{Effects} \mathrm{of}$ cyclosporine, tacrolimus and rapamycin on renal calcium transport and vitamin D metabolism. Am J Nephrol. 2011;34:87-94.

11. Maalouf NM, Shane E. Osteoporosis after solid organ transplantation. J Clin Endocrinol Metab. 2005;90:2456-65.

12. Courbebaisse M, Souberbielle JC, Thervet E. Potential nonclassical effects of vitamin D in transplant recipients. Transplantation. 2010;89:131-7. 
13. Akeno N, Matsunuma A, Maeda T, et al. Regulation of vitamin D-1alpha-hydroxylase and -24-hydroxylase expression by dexamethasone in mouse kidney. $\mathrm{J}$ Endocrinol. 2000;164:339-48.

14. Aris RM, Lester GE, Renner JB, et al. Efficacy of pamidronate for osteoporosis in cystic fibrosis patients following lung transplantation. Am J Respir Crit Care Med. 2000;162:941-6.

15. Stephenson A, Brotherwood M, Robert R, et al. Increased vitamin $\mathrm{A}$ and $\mathrm{E}$ levels in adult cystic fibrosis patients after lung transplantation. Transplantation. 2005;79:613-5.

\title{
S2.4 \\ EXTRA-SKELETAL IMPACT OF VITAMIN D
}

\author{
Lena E. Hjelte, M.D., Ph.D. \\ Stockholm CF Center, Dept. of Pediatrics, Karolinska Univ. Hospital Huddinge, Stockholm, Sweden
}

Classical function of the active form 1,25-dihydroxyvitamin $\mathrm{D}$ is maintaining calcium homeostasis by stimulating calcium transport from intestine and kidney into the blood, and by having direct negative feedback effect on parathormone release. However, several recent observations have initiated a re-evaluation of the physiologic and pharmacologic actions of vitamin $\mathrm{D}$. The nuclear vitamin $\mathrm{D}$ receptor has been found to be expressed in most tissues in the body, and CYP27B1, the enzyme required to convert circulating $25(\mathrm{OH}) \mathrm{D}$ to 1,25 -dihydroxyvitamin $\mathrm{D}$, has been discovered in several cell types, including immune cells and epithelial cells $(1,2)$. This has been followed by subsequent description of "non-classical functions of vitamin D," which comprise anti-proliferative, differentiation-regulating, anti-diabetic, and immunomodulatory properties of vitamin $\mathrm{D}$, and are so far firmly supported by numerous in vitro and in vivo animal experiments (3).

The in vitro findings of immunomodulatory properties of vitamin D consistently suggest that vitamin D stimulates the innate immune system and enhances antimicrobial activity at early stages of infection $(4,5)$, but provides negative feedback mechanism at later stages of infection (6). The inhibitory effects of vitamin D on adaptive immune response include induction of the potent antiinflammatory cytokine IL-10. This complex immunomodulatory role of vitamin D has been reviewed in detail (1). So far there are no large human studies on the proposed role of vitamin $\mathrm{D}$ in the immune system during infection.

The vast majority of children and adults with CF have serum levels of 25-hydroxyvitamin D $(25(\mathrm{OH}) \mathrm{D})$ below $30 \mathrm{ng} / \mathrm{mL}(75 \mathrm{nmol} / \mathrm{L})$, the upper cut-off value for vitamin D insufficiency, despite following the currently recommended vitamin D supplementation regimens $(7,8)$.

We hypothesized that chronic vitamin D insufficiency may contribute to the ongoing CF lung inflammation, which is associated with high IgG levels, low IL-10 levels, and poor lung function and studied the relation between vitamin $\mathrm{D}$ and serum $\mathrm{IgG}$, and, between vitamin $\mathrm{D}$ and lung function (9). The study was done in a large, well-characterized Scandinavian CF population. Serum $25(\mathrm{OH}) \mathrm{D}$ and total IgG were measured, spirometry car- ried out and vitamin D intake data gathered using a seven-day dietary food record. Multiple linear regression analyses were performed for IgG and FEV1 as dependent variables, and serum $25(\mathrm{OH}) \mathrm{D}$, daily food and supplemented vitamin D sources of intake as independent variables. The model was controlled for age, gender, genotype, CF-related diabetes, infection/colonization status, long-term oral corticosteroid treatment, long-term treatment with macrolides, pancreatic insufficient vs. sufficient phenotype and Body Mass Index z-score. Serum total $\mathrm{IgG}$ levels were negatively associated with serum 25(OH)D $\left(\mathrm{R}^{2}=0.386\right.$; beta=-5.3; $\left.\mathrm{p}<0.001\right)$, supplemented vitamin $\mathrm{D}$ intake per $\mathrm{kg}$ bodyweight $\left(\mathrm{R}^{2}=0.388\right.$; beta $=$ $2.1 ; \mathrm{p}<0.001)$, and total vitamin $\mathrm{D}$ intake per kg bodyweight $\left(R^{2}=0.415\right.$; beta $\left.=-1.5 ; p=0.002\right)$. Serum $25(\mathrm{OH}) \mathrm{D}$ was positively associated with FEV1 $\left(\mathrm{R}^{2}=0.318\right.$; beta $=0.16 ; \mathrm{p}=0.028$ ). The study supports the proposed role of vitamin $\mathrm{D}$ in the immune system during infection and substantiates prospective studies.

The Third National Health and Nutrition Examination Survey (14 000 noninstitutionalized adults) revealed a significant association between $25(\mathrm{OH}) \mathrm{D}$ levels and pulmonary function (10). The effect that $25(\mathrm{OH}) \mathrm{D}$ levels in patients with chronic lung disease may have on pulmonary function has not been extensively studied. However, vitamin D has been linked in some studies with atopy- and asthma-associated phenotypes in children with established disease. Recently it has been shown that children with inadequate vitamin D levels are at increased risk of developing atopy, and subsequently bronchial hyperresponsiveness and asthma (11). Low vitamin D levels at age 6 was a predictor of atopy and asthma at 14.

Vitamin D may treat or prevent allergy to Aspergillus in patients with $\mathrm{CF}$, according to the results of a recent in vitro study (12). Using peripheral CD4+ T cells isolated from patients with ABPA, the investigators found that the costimulatory molecule OX40 ligand (OX40L) was critical in driving Th2 allergic responses to A. fumigatus, whereas CD4+ T cells from patients without ABPA did not mount enhanced $\mathrm{Th} 2$ responses and contained more regulatory $\mathrm{T}$ cells expressing transforming growth factor 
beta (TGF- $\beta$ ). In patients with ABPA, increased Th2 reactivity was correlated with lower mean serum levels of vitamin D. Adding 1,25 OH-vitamin D3 to cells from patients with ABPA markedly decreased expression of OX40L, increased expression of TGF- $\beta$ needed for development of allergen tolerance, increased Treg TGF$\beta$ expression, and decreased Th2 responses. Also, $1,25(\mathrm{OH}) 2 \mathrm{D} 3$ has been shown to induce increases in antimicrobial proteins and secretion of antimicrobial activity against pathogens including Pseudomonas aeruginosa (13). 1,25(OH)2 D3 thus directly regulates antimicrobial peptide gene expression, revealing the potential of its analogues in treatment of opportunistic infections.

Vitamin D supplementation has been associated with a decreased incidence of type 1 diabetes (14) and plasma $25(\mathrm{OH}) \mathrm{D}$ has been described to be associated with type 2 diabetes (15). The exact molecular pathways are not completely understood but vitamin $\mathrm{D}$ has been suggested to act via its immunomodulatory effects in type 1 diabetes (16-17) and to increase insulin secretion and peripheral insulin sensitivity in type 2 diabetes (18-19). We assessed the relationship between vitamin $\mathrm{D}$ and $\mathrm{CF}$ related diabetes, glucose tolerance and HbA1c in 898 Scandinavian CF patients. Vitamin D insufficiency degree $(\mathrm{OR}=1.36 ; \mathrm{p}=0.032)$ and serum $25(\mathrm{OH}) \mathrm{D}<30$ $\mathrm{nmol} / \mathrm{L}(\mathrm{OR}=1.79 ; \mathrm{p}=0.042)$ were significant risk factors for CF-related diabetes. Accordingly, $\log _{\mathrm{e}}-\mathrm{HbA} 1 \mathrm{c}$ value was positively associated with serum $25(\mathrm{OH}) \mathrm{D}<$ $30 \mathrm{nmol} / \mathrm{l}$ and $<50 \mathrm{nmol} / \mathrm{l}$, as well as with vitamin D insufficiency degree $\left(\operatorname{adj} . \mathrm{R}^{2}=20.5 \%\right.$ and $\mathrm{p}<0.05$ in all). In subgroup analyses, serum $25(\mathrm{OH}) \mathrm{D}<30 \mathrm{nmol} / \mathrm{l}$ determined the $\log _{\mathrm{e}}-\mathrm{HbA} 1 \mathrm{c}$ value in paediatric patients (adj. $\left.\mathrm{R}^{2}=20.2 \% ; \mathrm{p}=0.017\right)$, but not in adults. Improving vitamin $\mathrm{D}$ status may have some anti-diabetic effect in $\mathrm{CF}$, especially in children. Prospective studies on the proposed role of vitamin D insufficiency in the pathophysiology of diabetes mellitus are needed.

References: 1. Mora JR, IwataM, von Andrian UH. Vitamin effects on the immune system: vitamins A and D take centre stage. Nat Rev Immunol. 2008;8:685-98.

2. Hansdottir S, Monick MM, Hinde SL, et al. Respiratory epithelial cells convert inactive vitamin $\mathrm{D}$ to it active form: potential effects on host defense. J Immunol. 2008;181:7090-9.

3. Bouillon R, Carmeliet G, Verlinden L, et al. Vitamin $\mathrm{D}$ and human health: lessons from vitamin $\mathrm{D}$ receptor null mice. Endocrin Rev. 2008;29:726-76.

4. Liu PT, Stenger S, Li H, et al. Toll-like receptor triggering of a vitamin D-mediated human antimicrobial response. Science. 2006;311:1770-3.

5. Urry Z, Xystrakis E, Richards DF, et al. Ligation of TLR9 induced on human IL-10-secreting Tregs by 1alpha,25dihydroxyvitamin D3 abrogates regulatory function. J Clin Invest. 2009;119:387-98.

6. Sadeghi K, Wessner B, Laggner U, et al. Vitamin D3 down-regulates monocyte TLR epression and triggers hyporesponsiveness to pathogen-associated molecualr patterns. Eur J Immunol. 2006;36:361-70.

7. Stephenson A, Brotherwood M, Robert R, et al. Cholecalciferol significantly increases 25-hydroxyvitamin D concentrations in adults with cystic fibrosis. Am J Clin Nutr. 2007;85:1307-11.

8. Rovner AJ, Stallings VA, Schall JI, et al. Vitamin D insufficency in children, adolescents, and young adults with cystic fibrosis despite routine oral supplemenation. Am J Clin Nutr. 2007;86:1694-9.

9. Pincikova T, Nilsson K, Moen IE, et al. Inverse relation between vitamin D and serum total $\mathrm{IgG}$ in the Scandinavian Cystic Fibrosis Nutritional Study. Eur J Clin Nutr. 2011;65:102-9.

10. Black PN, Scragg R. Relationship between serum 25-hydroxyvitamin $\mathrm{D}$ and pulmonary function in the third national health and nutrition examination survey. Chest. 2005;128:3792-8.

11. Hollams EM, Hart PH, Holt BJ, et al. Vitamin D and atopy and asthma phenotypes in children: a longitudinal cohort study. Eur Respir J. 2011 May 12. Published online before print.

12. Kreindler JL, Steele C, Nguyen N, et al. Vitamin D3 attenuates Th2 responses to Aspergillus fumigatus munted by $\mathrm{CD}+\mathrm{T}$ cells from cystic fibrosis patients with allergic bronchopulmonary aspegillosis. J Clin Invest. 2010;120:3242-54.

13. Wang TT, Nestel FP, Boureau V, et al. 1,25-Dihydroxyvitamin D3 is a direct inducer of antimicrobial peptide gene expression. J Immunol. 2004;173:2909-12.

14. Hyppönen E, Läärä E, Reunanen A, et al. Intake of vitamin $\mathrm{D}$ and risk of type 1 diabetes: a birth-cohort study. Lancet. 2001;358:1500-3.

15. Pittas AG, Sun Q, Manson JE, et al. Plasma 25hydroxyvitamin D concentration and risk of incident type 2 diabetes in women. Diabetes Care. 2010;33:20213.

16. Danescu LG, Levy S, Levy J. Vitamin D and diabetes mellitus. Endocrine. 2009;35:11-7.

17. Jeffery LE, Burke F, Mura M, et al. 1,25-Dihydroxyvitamin D3 and IL-2 combine to inhibit T cell production of inflammatory cytokines and promote development of regulatory $\mathrm{T}$ cells expressing CTLA-4 and FoxP3. J Immunol. 2009;183:5458-67.

18. Chiu KC, Chu A, Go VL, et al. Hypovitaminosis $\mathrm{D}$ is associated with insulin resistance and beta cell dysfunction. Am J Clin Nutr. 2004;79:820-5.

19. Teegarden D, Donkin SS. Vitamin D: emerging new roles in insulin sensitivity. Nutr Res Rev. 2009;22:82-92. 


\title{
S3.1 \\ BEYOND EVIDENCE-BASED MEDICINE: THE LIMITS OF EVIDENCE- BASED MEDICINE, AND WHAT TO DO
}

\author{
Mark R. Tonelli, M.D., M.A. \\ University of Washington, Seattle, WA, USA
}

Evidence-based medicine (EBM) has been championed as a "new paradigm" for medical education and practice (1). EBM focuses on medical knowledge derived from controlled clinical trials, asserting that such knowledge represents the "best evidence" that can be used to support a clinical decision. The value of clinical research for clinical medicine is limited, however, by the relative lack of research, the relevance of the research to clinical practice and by the difficulty of applying populationbased knowledge to the treatment of individuals. Other forms of medical knowledge, including those derived from pathophysiologic understanding and clinical experience, might be helpful, but have been devalued by EBM (2). Though acknowledging the need to integrate various kinds of medical and non-medical knowledge into clinical decision making, proponents of EBM have said very little about how such integration should actually take place. The process by which clinicians are to weigh and balance what may be conflicting information coming from not only published clinical research but also from personal experience, pathophysiologic understanding of disease, the preferences of individual patients or other "non-evidentiary" sources has not been fully elucidated.

Five topic areas are relevant to any clinical decision (Table) (3). Recognizing that each form of medical knowledge (empirical, experiential and pathophysiologic) has significant strengths and limitations when it comes to utilizing that knowledge in the care of individual patients, hierarchies of knowledge advanced by EBM cannot be helpful in deciding on individual treatment decisions. Rather, the clinician must weigh all relevant information in attempting to come to the best decision for an individual patient. The process of clinical decision making must begin with the patient at hand, with the clinician then considering all empirical, experiential and pathophysiologic facts and reasons that bear upon the case before reaching a conclusion regarding the most medically appropriate course of action. Patient preferences and system features need to be factored in at this point in order to finalize a treatment choice or recommendation.

The care of persons with cystic fibrosis has greatly benefited from high-quality clinical research performed over the last several decades. CF care, however, requires individualization. Many treatments in CF (e.g., pancreatic enzyme replacement) are not supported by high-quality clinical research yet are clearly defensible as best practice. New therapeutic interventions (e.g., chronic azithromycin) can be expected to work for some with $\mathrm{CF}$, but not all. Clinicians may find it appropriate to expand the use of approved therapies (e.g., TOBI) to patients who would not have been eligible for study participation or for indications not subjected to clinical investigation. Finally, not all clinical research demonstrating effectiveness of an intervention will be compelling enough to change practice. Features beyond the study design must be considered. When interventions not well supported by clinical research results are advocated by experts or individual practitioners, the five topics outlined can serve as framework, making the reasoning explicit and open to rebuttal.

The optimal practice of clinical medicine, though requiring the knowledge of the results of clinical research, demands that clinicians attempt to deliver the best care to a particular individual. This cannot be accomplished by an appeal to clinical research results alone.

The Five Topics of Clinical Decision Making

Empirical: Derived from clinical research.

Experiential: Derived from personal clinical experience or the clinical experience of others (i.e., expert opinion).

Pathophysiologic Rationale: Based on underlying theories of physiology, disease and healing.

Patient Values and Preferences: Derived from personal interaction with individual patients.

System Features: Including resource availability, societal and professional values, legal and cultural concerns.

References: 1 . The Evidence-Based Medicine Working Group. Evidence-based medicine: A new approach to teaching the practice of medicine. JAMA. 1992;268:2420-5.

2. Tonelli MR. The philosophical limits of evidencebased medicine. Acad Med. 1998;73:234-40.

3. Tonelli MR. Integrating evidence into clinical practice: an alternative to evidence-based approaches. J Eval Clin Pract. 2006;12:248-56. 


\title{
S3.2 \\ PRIORITIZING CHRONIC PREVENTIVE PULMONARY THERAPIES
}

\author{
Patrick A. Flume, M.D. \\ Medical Univ. of South Carolina, Charleston, SC, USA
}

A clinical practice guideline is a systematically developed statement designed to assist practitioners and patients in making decisions about appropriate health care for specific circumstances (1). The Cystic Fibrosis Foundation has invested considerable resources into the development of guidelines for the diagnosis and management of cystic fibrosis (CF) and its complications. As these guidelines are only recently published, it is premature for us to ask whether these guidelines have had a meaningful effect in the lives of patients with $\mathrm{CF}$, but in principle, if clinicians provide interventions which are known to improve clinical outcomes, then patients should benefit (2).

The development of guidelines alone is not sufficient for there to be benefit. The critical "next step" is the implementation of those guidelines. A lesson learned from guidelines in other disease conditions is that clinicians often don't utilize them. Studies have demonstrated that patients receive only about $50 \%$ of recommended evidence-based therapies, and mostly because of a lack of using recommended therapies (3). The key factors of successful guidelines are: they should cover an area of practice which varies greatly and clinicians are aware of this; their evidence is fairly secure; the indication for their use is common in the practice of the targeted clinicians; clinicians are aware of the gaps in their own knowledge about the clinical condition covered by the guidelines; and the benefits of implementation are large (2). For patients this means that the change will improve outcomes and for clinicians, the care should be quicker or easier.

We can use the guidelines on the use of chronic medications to maintain lung health (4) as an example of guideline development and implementation. These guidelines were meant to address an area of great importance to the CF community. The CFF Patient Registry (CFFPR) had demonstrated to us that there was wide variation in the use of such medications across the center network (5). The development of the guidelines followed strict methodologies to ensure sound recommendations based on the evidence. A committee constructed the relevant questions, a systematic review of the literature was performed, and there was an a priori plan of how to assess the evidence.

The CF community was asked to offer commentary prior to submission for publication and the manuscript reporting the guidelines were subject to peer review. The $\mathrm{CFF}$ then invested resources into the education of the $\mathrm{CF}$ community regarding the guidelines including webinars and printed materials.
Since that time, there has been additional evidence that supports the recommendations provided in the guidelines. Analyses of the CFFPR have shown a decreased hospitalization rate in those patients on chronic macrolides over 2 years (6), a reduced mortality in those patients using inhaled tobramycin and/or dornase alfa (7), and a slower rate of decline of FEV1 in patients using inhaled antibiotics (8). The Canadian Study of High-Dose Ibuprofen in CF was under-powered and yet still found a reduced rate of decline of FVC but not FEV (9), and an analysis of ibuprofen usage in the CFFPR found a $23 \%$ reduction in rate of decline of lung function in treated compared to those not treated (10). Novel analyses of adherence to medications have shown a lower rate of hospitalization (11) and a reduced occurrence of exacerbations (12).

Just as the development of the guidelines was based upon a systematic approach, the implementation of guidelines is facilitated by a systematic approach. Success is often based upon assuring that all patients receive the therapy. This can be accomplished using a quality improvement approach that engages the clinical team, patients and families, educating all to be sure there is agreement, and then executing an action plan to make these guidelines part of standardized care. A successful implementation of the chronic medications guidelines has been demonstrated at one center where prescription of medications increased from $\sim 60 \%$ to $\sim 90 \%$ of eligible patients (13).

Such reports are encouraging, but there remains one additional challenge acknowledged in the guidelines. We should not presume that all patients should be treated with all recommended therapies, so how should we introduce therapies to our patients? In addition, there has been an increasing trend by clinicians to introduce therapies earlier under the premise that this will better preserve lung function in the long run. The evidence supporting the medications included in the guidelines primarily demonstrated improvement in lung function (i.e., $\mathrm{FEV}_{1}$ ), but we recognize that changes in $\mathrm{FEV}_{1}$ are a relatively late event of the ongoing pathogenesis of CF lung disease. So if we choose to intervene earlier, how do we rationally institute a practice change? What are the appropriate diagnostic tests and how will we measure success?

There is one diagnostic test that has already established a consistent place in the evaluation and treatment of CF patients - periodic culture of the oropharynx. The practice of antibiotic treatment of early Pseudomonas infection has become fairly standard among CF centers. 
This is done with the belief that early infection can be eradicated and the time to establishment of chronic infection can be delayed. Once chronic infection has been established, the use of chronic aerosolized antibiotics should be used as recommended in the guidelines. Such an approach is well-accepted for the treatment of Pseudomonas, and there is increased interest in a similar approach to other bacterial pathogens.

Whereas we have a clear diagnostic test to guide the use of inhaled antibiotics, what about the other medications recommended in the guidelines? We recognize that the rate of disease progression is variable among patients; there are patients with milder manifestations of disease such that many have evaded diagnosis until later in life. Some have suggested that computed tomography (CT) of the chest or bronchoscopy might provide information that could guide therapy, but these come with concerns of increased cost, potential toxicity, and an increased treatment burden and they have not found a place in standard practice. Epidemiologic studies have shown the relationship between early nutritional status (e.g., weight and height for age percentiles) and eventual pulmonary health (14). Such observations may provide the needed information about when to introduce therapies (even long before symptoms), but they do not demonstrate which therapy to introduce first. We can assess tolerance of the medication, but assessment of benefit will be more challenging to prove. In addition, we must consider the possibility of unintended consequence that could occur with therapies.

In conclusion, it is likely that the clinical practice guidelines will provide benefit to patients, but only if they are followed. That is, the clinicians must prescribe the medications and the patients must adhere to a treatment regimen. The key to success of implementation of the recommendations contained within the guidelines is to develop a systematic approach as in a quality improvement project until they become standardized therapy. For those patients who are not yet on therapies, a systematic approach should be used to assess, diagnose and treat with these therapies. It is highly unlikely that formal clinical trials will be performed that will answer the question as to which therapy first and when, and so the CF community must standardize their approach and share their results. Only in these comparative effectiveness studies will we be able to know the optimal approach with greater certainty.

References: 1. Field MJ, Lohr KN, eds. Guidelines for Clinical Practice: From Development to Use. Washington DC. Institute of Medicine, National Academy Press, 1992.

2. Bazian Ltd. Do evidence-based guidelines improve the quality of care? Evid Based Healthc Public Health. 2005;9:270-5.

3. McGlynn EA, et al. The quality of health care delivered to adults in the United States. N Engl J Med. 2003;348:2635-45.

4. Flume PA, et al. Cystic fibrosis pulmonary guidelines: chronic medications for maintenance of lung health. Am J Respir Crit Care Med. 2007;176:957-69.

5. Cystic Fibrosis Foundation Patient Registry. Annual Data Report to the Center Directors. Bethesda, MD: Cystic Fibrosis Foundation; 2010.

6. West NE, et al. The effect of chronic macrolide therapy in cystic fibrosis patients infected with Pseudomonas aeruginosa. Pediatr Pulmonol. 2010;Suppl 33:333.

7. Sawicki GS, et al. Use of inhaled tobramycin is associated with reduced mortality in patients with cystic fibrosis. Pediatr Pulmonol. 2010;Suppl 33:363.

8. VanDyke R, et al. Treatment with tobramycin and the rate of decline in lung function in CF: an instrumental variables approach. Pediatr Pulmonol. 2010;Suppl 33:375.

9. Lands LC, et al, High-dose ibuprofen in cystic fibrosis: Canadian safety and effectiveness trial. J Pediatr. 2007;151:249-54.

10. Konstan MW, et al. Clinical use of ibuprofen is associated with slower FEV1 decline in children with cystic fibrosis. Am J Respir Crit Care Med. 2007;176:1084-9.

11. Briesacher BA, et al. Adherence with tobramycin inhaled solution and health care utilization. BMC Pulm Med. 2011;11:5.

12. Eakin $\mathrm{MN}$, et al. Longitudinal association between medication adherence and lung health in people with cystic fibrosis. J Cyst Fibros. 2011;10:258-64.

13. McPhail GL, et al. Improving evidence-based care in cystic fibrosis through quality improvement. Arch Pediatr Adolesc Med. 2010;164:957-60.

14. Konstan MW, et al. Growth and nutritional indexes in early life predict pulmonary function in cystic fibrosis. J Pediatr. 2003;142:624-30. 


\title{
S3.3 \\ UP THE CYSTIC FIBROSIS PULMONARY CREEK IN A BARBED WIRE CANOE
}

\author{
Andrew Bush, M.D., FRCP, FRCPCH \\ Paediatric Respiratory Medicine, Imperial College \& Royal Brompton Hospital, London, United Kingdom
}

Although cystic fibrosis (CF) has an increasingly good prognosis, early death is still too common. This review focuses in particular on what we can learn from severe asthma; most asthmatic children respond to low doses of inhaled corticosteroids, and detailed protocols have been described to rationalise management (1). In summary, the umbrella term used at the start of the process is "problematic, severe asthma" (2) and the steps are to determine:

1. Is the diagnosis correct ("not asthma at all")?

2. Are there significant co-morbidities ("asthma plus")?

3. Is it "difficult" asthma (improves if the basics are got right)?

4. Is it true severe, therapy resistant asthma, which remains a problem even when the basics are got right, and therefore may be treated with "beyond the guidelines" therapy?

The hypothesis is that a similar approach may be useful when pulmonary $\mathrm{CF}$ is apparently very severe. There is no evidence to guide us in what we term "challenging CF"; what follows is personal practice based on our experience with asthma.

Definition of "challenging CF." These are arbitrary (as in severe paediatric asthma), and the following definitions are suggested. Children may become challenging very quickly (e.g., failure to improve after two or more courses of intravenous antibiotics in quick succession) or gradually, with a series of almost subclinical deteriorations.

- Any child with lung function $>2 \mathrm{Z}$-scores below CF charts (3)

- Any child who receives $>3$ courses of intravenous antibiotics annually (whether elective or unplanned)

- Any child requiring home oxygen

- Any child in "nutritional failure" (e.g., body mass index $(\mathrm{BMI})>2 \mathrm{Z}$ scores below the mean; drop in weight or body mass index (BMI) centiles by $10 \%$ over a year)

- Any child with a severe CF pulmonary complication (e.g., massive hemoptysis, pneumothorax, therapy resistant ABPA, oral steroid dependent)

- Any child whose self- or parent-reported symptoms are significantly overestimated or underestimated

- Any child in whom there is refusal or extreme reluctance to give prescribed treatment by the caregivers

Challenging CF: Is it really CF? In most cases the diagnosis will have been firmly established. It is at least worth considering whether another diagnosis with a specific therapy may have been missed.
Challenging CF: Is it CF plus? Of the comorbidities which may complicate asthma, obesity and food allergy are not likely relevant in CF. The relationship between upper and lower airways is complex (4), and severe rhinosinusitis should be sought and treated. Gastroesophageal reflux (GER) and dysfunctional breathing patterns (e.g., vocal cord dysfunction) may both complicate CF. GER may be difficult to diagnose, lipid laden macrophages have a good negative but a poor positive predictive value, measurement of pepsin in lavage may be helpful (5).

It is CF, comorbidities have been excluded as far as possible, now what? The next step is a detailed multidisciplinary assessment, which includes a home visit. By analogy with asthma (6), the following issues are addressed:

- Medication adherence: relevant in more than half the asthmatics. Prescription records are obtained, whether the medications are accessible and in date, and whether an inappropriately young child is being left to take medications unsupervised is determined (7). Consider measuring blood levels of appropriate medications (prednisolone, theophylline, itraconazole, voriconazole) and using nebulisers or airway clearance devices with a microchip which records adherence.

- Chest physical therapy practice: if equipment is prescribed, is it available or obviously unused at the back of a cupboard?

- The home environment: exposure to tobacco smoke is checked by cotinine measurements, allergen sensitization and exposure is checked, especially the presence of mould or fungi.

- Psychosocial issues: most referrals to psychology in the context of asthma were made only after discussions in the home, where parents are more likely to talk about sensitive issues.

- Education: knowledge of $\mathrm{CF}$ and the treatments is re-checked.

After gathering these data, a multi-disciplinary team meeting decides whether further more detailed investigation is needed, or whether addressing the basic management steps is the way forward. Clearly if standard therapies such as rhDNase, hypertonic saline and macrolides are not optimally deployed, then this should happen before invasive investigations are undertaken.

The next step: detailed investigation. Nutrition and pulmonary status are so tightly bound up that both should be evaluated; the detail of nutritional assessment will 
depend on BMI. Some of the tests may already have been done in the immediate past, and may therefore not need repeating.

- Assessment of lung structure and function: inspiratory and expiratory CT scan, full lung function including bronchodilator reversibility, and lung clearance index.

- Fibre optic bronchoscopy, broncho-alveolar lavage and endobronchial biopsy to exclude infection with atypical organisms, airway malacia and other unexpected structural disease, and determine the pattern of airway inflammation. Consider 16s rRNA studies and anaerobic culture, although results may be difficult to interpret. Induced sputum may be a complementary investigation.

- Exclusion of GER by pH monitoring, give consideration to an impedance study, and refer to gastro-enterology if any doubt.

- Exclude unexpected nocturnal sleep disordered breathing with polysomnography.

- Exclude insulin deficiency: random blood sugar monitoring, oral glucose tolerance test, continuous monitoring (CGMS).

- If there is concomitant nutritional failure, a full assessment by the dietitian including a 3 day fecal fat, celiac screen, stool culture for Giardia, and urinary electrolytes. A referral to gastroenterology is made if there is still diagnostic doubt.

What next? Unlike in asthma, there is insufficient evidence to be dogmatic about the outcome of this sort of process. Clinical scenarios we have encountered include:

- "Distal and dry" bad lung disease - distal airway disease with air trapping on inspiratory and expiratory CT scans, little sputum and minimal airway secretions on bronchoscopy. GER has to be excluded, and therapeutic choices include pulsed methyl prednisolone (which perhaps is underused because of fears of adverse effects), intravenous immunoglobulin (8) and other immunosuppressive agents.

- "Pan-airway and productive" - bad proximal bronchiectasis and marked purulent airway secretions. We use rotating nebulised antibiotics (TOBI ${ }^{\mathrm{TM}}$ and colistin month on month off of each; there may be a role for AZLI $^{\mathrm{TM}}$ here); planned three-monthly courses of intravenous antibiotics; and even long term home intravenous colistin.

- "Inflammation predominant" - Very occasional responses to methotrexate and cylosporin have been described on an anecdotal basis (9-12). Prior to an attempted trial of these agents, a most careful evaluation of more conventional approaches is mandated. Immunosuppression brings with it the risk of invasive infections such as aspergillosis (13).

- Psychological issues which are of course part of non-adherence, but there is much more to it than this. In particular in the teenage years, a lot of work may be needed. Issues include breakdown of family relationships and the use of non-adherence as a weapon; "over- calling" the child's symptoms; dysfunctional breathing patterns.

Challenging CF: Conclusions. We propose the use of a protocol based on that used in really severe asthma. In the context of asthma, we have learned that much "nightmare asthma" is readily amenable to basic management if implemented effectively. We hypothesise that the use of a similar protocol will identify patients with CF who need closer attention to conventional management, and a subgroup who will require innovative therapies.

References: 1. Bush A, Saglani S. Management of severe asthma in children. Lancet. 2010;376:814-25.

2. Bush A, Hedlin G, Calsen K-H, et al. Severe childhood asthma: a common international approach? Lancet. 2008;372:1019-21.

3. Kulich M, Rosenfeld M, Campbell J, et al. Diseasespecific reference equations for lung function in patients with cystic fibrosis. Am J Respir Crit Care Med. 2005;172: 885-91.

4. de Benedictis FM, Bush A. Rhinosinusitis and asthma: epiphenomenon or causal association? Chest. 1999;115:550-6.

5. Bracken MB, Fleming L, Hall P, et al. The importance of nurse led home visits in the assessment of children with problematic asthma. Arch Dis Child. 2009;94:780-4.

6. McNally P, Ervine E, Shields MD, et al. High concentrations of pepsin in bronchoalveolar lavage fluid from children with cystic fibrosis are associated with high interleukin-8 concentrations. Thorax. 2011;66:140-3.

7. Orrell-Valente JK, Jarlsberg LG, Hill LG, et al. At what age do children start taking asthma medicines on their own? Pediatrics. 2008;122:e1186-92.

8. Balfour-Lynn IM, Mohan U, Bush A, et al. Intravenous immunoglobulin for cystic fibrosis lung disease: a case series of 16 children. Arch Dis Child. 2004;89:315-9.

9. Ballmann M, Junge S, von der Hardt H. Lowdose methotrexate for advanced pulmonary disease in patients with cystic fibrosis. Respir Med. 2003;97:498-500.

10. Prescott WA Jr, Johnson CE. Antiinflammatory therapies for cystic fibrosis: past, present, and future. Pharmacotherapy. 2005;25:555-73.

11. Jaffe A, Balfour-Lynn IM. Treatment of severe small airways disease in children with cystic fibrosis: alternatives to corticosteroids. Paediatr Drugs. 2002;4:381-9.

12. Bhal GK, Maguire SA, Bowler IM. Use of cyclosporin A as a steroid sparing agent in cystic fibrosis. Arch Dis Child. 2001;84:89.

13. Brown K, Rosenthal M, Bush A. Fatal invasive aspergillosis in an adolescent with cystic fibrosis. Pediatr Pulmonol. 1999;27:130-3. 


\title{
S3.4 \\ NONTUBERCULOUS MYCOBACTERIA: WHO TO TREAT, HOW TO TREAT, WHEN TO STOP
}

\author{
Jerry A. Nick, M.D. ${ }^{1,2}$, Michelle Nichols, M.D. ${ }^{1,2}$, Cathy Chacon ${ }^{2}$, Sara J. Brayshaw ${ }^{1}$, Marion Jones ${ }^{3}$, Christine \\ Barboa $^{1}$, David P. Nichols ${ }^{1}$, Jennifer S. Janssen ${ }^{1,2}$, Milene T. Saavedra ${ }^{1,2}$, Jennifer L. Taylor-Cousar, M.D. ${ }^{1,2}$, Scott D. \\ Sagel, M.D. ${ }^{2,3}$ and Stacey L. Martiniano, M.D. ${ }^{2,3}$ \\ 1. National Jewish Health, Denver, CO, USA; 2. Univ. of Colorado Denver, Aurora, CO, USA; 3. Children's \\ Hospital, Aurora, CO, USA
}

Nontuberculous mycobacteria (NTM) are ubiquitous in the environment existing as biofilms, aerosols, or dusts. NTM are extraordinarily hardy, and can withstand standard disinfectants, chlorine, glutaraldehyde, mercury, and formaldehyde. They have intrinsic resistance to broad classes of frontline antibiotics used for the treatment of bacteria. NTM survive under a wide range of conditions, and have been isolated from distilled water, hospital water heated to $55^{\circ} \mathrm{C}$, ice machines, public drinking water and even showerheads. Therefore, it is not surprising that these organisms are often present in sputum from patients with cystic fibrosis $(\mathrm{CF})(1)$.

In the setting of CF lung disease, the significance of NTM in respiratory secretions is a major diagnostic and therapeutic challenge. NTM have traditionally been categorized by the Runyon classification. In this system, organisms from group III (slow-growing), such as $M$. avium complex (MAC), and group IV (rapid-growing), such as M. abscessus, appear most commonly as CF pathogens. The incidence of NTM disease appears to be increasing in $\mathrm{CF}$, and also in other lung diseases. Increased recovery of NTM in CF airway samples is due in part to specialized processing techniques that have reduced overgrowth by bacterial co-infections, as well as increasing physician awareness. The possibility also exists that exposure to NTM is becoming more common in our modern environment. However, acquisition of NTM is clearly an age-related complication of CF (2), and as median survival increases, so will the prevalence of NTM infection.

Who to treat. When NTM is first recovered from a CF respiratory sample, thorough consideration is needed to determine if treatment is required. Not uncommonly, NTM is recovered once, and is not seen in subsequent cultures (2). In other patients, NTM is recovered on multiple occasions, but with no apparent clinical consequences (3). However, in some patients, NTM is clearly associated with accelerated progression of lung disease. As the usual signs, symptoms, and radiographic features of NTM disease broadly overlap with CF lung disease, it is frequently difficult to determine when NTM is an active pathogen. Although Olivier et al. suggested an algorithm to diagnosis NTM disease in the setting of CF based on their nested cohort study, there have been no prospective trials to validate this algorithm (3). Important considerations include the species of NTM, quantity of organism, number of sep- arate positive cultures, clinical and radiographic evidence of disease progression, and exclusion of other causes of accelerated disease progression. Generally speaking, rapid growers such as M. abscessus are more virulent than slower growers such as M. avium (4). Nevertheless, slow growing NTMs are capable of causing disease in CF patients. Finally, it appears to us that NTM in children is more likely to have significant and sometimes devastating consequence, whereas an indolent clinical course is more often seen in older patients or those with an adult diagnosis.

The following diagnostic approach is one followed at the Colorado CF Center, and generally follows ATS consensus guidelines (5). A minimum of two positive cultures and radiographic progression must be present. However, the most important consideration is that clinical decline is unexpectedly rapid in the setting of aggressive treatment of known pathogens and comorbidities. Often NTM are first looked for (and found) in association with a clinical downturn. An essential part of determining the significance of a new NTM infection is to first aggressively treat other infecting pathogens such as $P$. aeruginosa or $S$. aureus infections with IV antibiotics. If the patient does not return to baseline with treatment, we consider a second course of antibacterial antibiotics. A thorough review of airway clearance and other basic elements of CF care should be performed, and therapies maximized. Finally, all potential comorbidities must be addressed, in particular new bacterial infections (i.e. B. cepacia), CF-related diabetes, poorly controlled sinusitis, chronic aspiration and ABPA. Through exclusion of these well-recognized causes of accelerated decline, the contribution of NTM can be established. The case for treatment should be presented to the patient and family, and it is important that both the caregiver and patient be convinced of the need for treatment, as therapy is often difficult and a variety of complications and side effects are likely. As part of the initiation of treatment, the patient should be made aware that there is no proven treatment strategy, the rate of success is not known, and even if treatment is successful the patient is at risk for reactivation or re-infection. However, in many cases treatment is clearly beneficial.

How to treat. In the absence of $\mathrm{CF}$ specific recommendations, an initial approach to treatment is based on guidelines developed for the general population (5). For M. abscessus, our standard approach is three-drug thera- 
py with parenteral amikacin and imipenem (or cefoxitin), combined with an oral macrolide for 3 months. Following this acute intensive treatment phase, therapy can often be changed to an oral macrolide and a fluoroquinolone, in combination with inhaled amikacin. For $M A C$, our standard approach is 3-drug therapy with a macrolide, ethambutol, and rifampin. However, in the presence of cavitary disease, amikacin can be used for the first 3 months of treatment, and continued up to 6 months if sputum cultures remain positive. Clearly, antibiotic selection will need to be individualized based on pre-existing allergies and toxicities. To a lesser extent, the antibiotic susceptibility of the organism may be a consideration as well; in particular the presence of resistance to clarithromycin in patients who received longterm therapy with azithromycin. Initially, the CF team needs to work closely with the patient to find a drug combination that is tolerated, and in some cases this approach is most effectively accomplished in the inpatient setting. Once a stable drug combination is identified, a critical consideration is monitoring of drug pharmacokinetics, as $\mathrm{CF}$-specific alterations in oral absorption and volume of distribution can result in unexpectedly low drug levels (6). In general, administration of oral agents with food and enzymes appears to enhance absorption, which may directly contradict recommendations for the general population (i.e. take on an empty stomach). Monitoring for drug-related toxicities is mandatory, such toxicities include development of ototoxicity associated with aminoglycosides, and visual changes associated with ethambutol. A CBC (with platelets) and liver function tests are checked at each visit to monitor for bone marrow suppression and hepatitis, which can be induced by a variety of the antimicrobials. Patients are typically seen in clinic every month during treatment, for sputum culture and laboratory analysis in addition to routine spirometry and physical exam. Standard CF care should be continually emphasized, especially aggressive airway clearance. Throughout the course of treatment, the potential for pulmonary exacerbation due to P. aeruginosa, $S$. aureus or other typical CF infections must be considered, even if the NTM treatment may have partial overlapping antimicrobial activity against these organisms.
When to stop treatment. As with other aspects of NTM treatment, the duration of therapy is not known. In non-CF lung disease, success is defined by clearing the sputum of organisms. The ATS recommendation is that treatment continue for 12 months following clearing of the sputum (5). In our experience, this endpoint is often difficult to achieve. As other biofilm-forming infections such as $P$. aeruginosa are not routinely cleared from the CF airway, it is expected that NTM infections are sometimes impossible to eradicate as well. However, as with $P$. aeruginosa, we have seen sustained clinical response to treatment following antimicrobial therapy, despite continued presence of NTM in sputum isolates. Our approach is to initially attempt to clear the sputum, with a prolonged (6-12 month) treatment course. If treatment is not well tolerated despite attempts to introduce alternative drug combinations, a shorter course of therapy can be offered, with clinical improvement as an endpoint. In all patients with a history of NTM, we monitor all subsequent cultures for NTM, expecting that additional courses of NTM treatment will be needed episodically.

References: 1. Nick JA. Nontuberculous mycobacteria in cystic fibrosis. Semin Respir Crit Care Med. 2003;24:693-702.

2. Olivier KN, Weber DJ, Wallace RJ Jr, et al. Nontuberculous mycobacteria. I: Multicenter prevalence study in cystic fibrosis. Am J Respir Crit Care Med. 2003;167:828-34.

3. Olivier KN, Weber DJ, Lee JH, et al. Nontuberculous mycobacteria. II: Nested-cohort study of impact on cystic fibrosis lung disease. Am J Respir Crit Care Med. 2003; $167: 835-40$.

4. Esther CR Jr, Esserman DA, Gilligan P, et al. Chronic Mycobacterium abscessus infection and lung function decline in cystic fibrosis. J Cyst Fibros. 2010;9:117-23.

5. Griffith DE, Aksamit T, Brown-Elliott BA, et al. An official ATS/IDSA statement: Diagnosis, treatment, and prevention of nontuberculous mycobacterial diseases. Am J Respir Crit Care Med. 2007;175:367-416.

6. Gilljam M, Berning SE, Peloquin CA, et al. Therapeutic drug monitoring in patients with cystic fibrosis and mycobacterial disease. Eur Respir J. 1999;14:347-51.

\title{
S4.1
}

\section{DOMAIN-DOMAIN INTERACTIONS IN THE FULL-LENGTH HUMAN CFTR PROTEIN REVEALED BY SINGLE PARTICLE ELECTRON MICROSCOPY AND ELECTRON CRYSTALLOGRAPHY}

\author{
Robert C. Ford, Ph.D. ${ }^{1}$, Mark F. Rosenberg ${ }^{1}$, Liam O’Ryan ${ }^{1}$, Guy Hughes ${ }^{1}$, Zhefeng Zhao $^{2}$, Luba Aleksandrov ${ }^{2}$ and \\ John R. Riordan, Ph.D. ${ }^{2}$ \\ 1. Faculty of Life Sciences, Univ. of Manchester, Manchester, United Kingdom; 2. Biochemistry \& Biophysics, Univ. \\ of North Carolina, Chapel Hill, NC, USA
}

The structure of CFTR probably resembles the structures of the bacterial efflux transporters Sav1866 $(1,2)$ and MsbA (3) and the mammalian drug transporter ABCB1 (4). This type of ABC transporter has $2 \times 6$ 
transmembrane alpha helices forming two transmembrane domains (TMDs) and these each have four long intracellular helical extensions of transmembrane helices which form cytoplasmic loops (ICLs). Four of these loops bind to pockets in the two cytoplasmic nucleotide binding domains (NBDs), with one loop from each transmembrane domain crossing over to contact the NBD on the opposite side of the transporter $(1,2)$. The structures of Sav1866, MsbA and ABCB1 show overall folds that are broadly similar, however ABCB1 (and some lowerresolution structures for other MsbA orthologs) show a wide separation of the NBDs and an inward-facing (i.e., cytoplasm-facing) orientation of the two TMDs $(3,4)$. In contrast, Sav1866 and some of the MsbA orthologs display closely associated NBDs and a more outward-facing configuration of the TMDs (1-3). These structural studies imply that major conformational changes can occur in the relative orientation of the four domains. Similar, but smaller conformational changes have also been observed in a separate group of bacterial ABC proteins which carry out the import of substances into the cell (5-11). Large conformational changes were previously proposed to be part of the substrate translocation mechanism for $\mathrm{ABC}$ transporters, and the formation of a sandwichdimer of NBDs in the presence of nucleotides will be addressed in more detail in my presentation (12).

Structural data derived from 2D crystals of CFTR have been published (13). These crystals were obtained in the presence of the non-hydrolysable ATP analogue AMP-PNP (13), and with two separate crystal forms present. The data generated by these studies was estimated to have a spatial resoilution of $\sim 20 \AA$, typical for negatively-stained specimens where the detail is limited by the granular nature of the uranyl acetate stain and by dehydration in the high vacuum of the microscope $(14,15)$. Despite these drawbacks, the structural data showed that CFTR within the $2 \mathrm{D}$ crystals was in a monomeric state, and that it was structurally homologous to ABCB1 (16), as expected. Conditions have now been found that yield better 2D arrays of CFTR and higher resolution data has been extracted from these crystals in the unstained and frozen-hydrated state (Rosenberg, M.F., et. al., submitted). These conditions require the absence of nucleotide, hence the protein should be quiescent. Under these conditions, the protein was packed in two crystalline layers stacked on top of each other. This new unit cell packing and the structures of the two CFTR molecules in the unit cell will be described in the symposium.

Single particle electron microscopy has been employed to study CFTR structure in the non-crystalline state. An early study of CFTR embedded in a heavy-atom stain reported dimeric complexes (17). A later study also reported dimeric complexes, although there was an unexpected void in the centre of the complex (18). Later, structural data were obtained for purified CFTR single particles in the unstained and frozen-hydrated state (19) and these data also showed dimeric CFTR complexes consistent with two side-by side molecules but with no large cavity in the centre of the dimer. Labelling of the CFTR particles using 1.8nm-diameter gold particles was used to establish domain-domain interactions (20), and provided the first direct $3 \mathrm{D}$ data on the location of the Rregion. I will describe the locations of the CFTR domains and discuss the correlation between the low-resolution domain labelling studies and the recent higher resolution 2D-crystal studies.

References: 1. Dawson RJ, Locher KP. Structure of a bacterial multidrug ABC transporter. Nature. 2006;443:180-5.

2. Dawson RJ, Locher KP. Structure of the multidrug ABC transporter Sav1866 from Staphylococcus aureus in complex with AMP-PNP. FEBS Lett. 2007;581:935-8.

3. Ward A, Reyes CL, Yu J, et al. Flexibility in the ABC transporter MsbA: Alternating access with a twist. Proc Natl Acad Sci USA. 2007;104:19005-10.

4. Aller SG, Yu J, Ward A, et al. Structure of P-glycoprotein reveals a molecular basis for poly-specific drug binding. Science. 2009;323:1718-22.

5. Davidson AL, Chen J. ATP-binding cassette transporters in bacteria. Annu Rev Biochem. 2004;73:241-68.

6. Hollenstein K, Frei DC, Locher KP. Structure of an $\mathrm{ABC}$ transporter in complex with its binding protein. Nature. 2007;446:213-6.

7. Kadaba NS, Kaiser JT, Johnson E, et al. The high-affinity $\mathrm{E}$. coli methionine $\mathrm{ABC}$ transporter: structure and allosteric regulation. Science. 2008;321:250-3.

8. Khare D, Oldham ML, Orelle C, et al. Alternating access in maltose transporter mediated by rigid-body rotations. Mol Cell. 2009;33:528-36.

9. Locher KP, Lee AT, Rees DC. The E. coli BtuCD structure: a framework for ABC transporter architecture and mechanism. Science. 2002;296:1091-8.

10. Pinkett HW, Lee AT, Lum P, et al. An inward-facing conformation of a putative metal-chelate-type ABC transporter. Science. 2007;315:373-7.

11. Oldham ML, Khare D, Quiocho FA, et al. Crystal structure of a catalytic intermediate of the maltose transporter. Nature. 2007;450:515-21.

12. Rosenberg MF, Velarde G, Ford RC, et al. Repacking of the transmembrane domains of P-glycoprotein during the transport ATPase cycle. Embo J. 2001;20:5615-25.

13. Rosenberg MF, Kamis AB, Aleksandrov LA, et al. Purification and crystallization of the cystic fibrosis transmembrane conductance regulator (CFTR). J Biol Chem. 2004;279:39051-7.

14. Ford RC, Holzenburg A. Electron crystallography of biomolecules: mysterious membranes and missing cones. Trends Biochem Sci. 2008;33:38-43.

15. Harris JR. Negative staining of thinly spread biological particulates. Methods Mol Biol. 1999;117:13-30. 
16. Rosenberg MF, Kamis AB, Callaghan R, et al. Three-dimensional structures of the mammalian multidrug resistance $\mathrm{P}$-glycoprotein demonstrate major conformational changes in the transmembrane domains upon nucleotide binding. J Biol Chem. 2003;278:8294-9.

17. Awayn NH, Rosenberg MF, Kamis AB, et al. Crystallographic and single-particle analyses of nativeand nucleotide-bound forms of the cystic fibrosis transmembrane conductance regulator protein. Biochem Soc Trans. 2005;33:996-9.

18. Mio K, Ogura T, Mio M, et al. Three-dimensional reconstruction of human cystic fibrosis transmembrane conductance regulator chloride channel revealed an ellipsoidal structure with orifices beneath the putative transmembrane domain. J Biol Chem. 2008;283:30300-10.

19. Zhang L, Aleksandrov LA, Zhao Z, et al. Architecture of the cystic fibrosis transmembrane conductance regulator protein and structural changes associated with phosphorylation and nucleotide binding. J Struct Biol. 2009; 167:242-51.

20. Zhang L, Aleksandrov LA, Riordan JR et al. Domain location within the cystic fibrosis transmembrane conductance regulator protein investigated by electron microscopy and gold labelling. Biochim Biophys Acta. 2010;1808:399-404.

\title{
S4.2 NBD FOLDING AND INTERACTIONS IN CFTR BIOSYNTHESIS
}

\author{
Patrick H. Thibodeau, Ph.D. \\ Dept. of Cell Biology, Univ. of Pittsburgh School of Medicine, Pittsburgh, PA, USA
}

As a member of the ABC-transporter superfamily of proteins, CFTR is composed of multiple modular protein domains. The CFTR functional complex is composed of a dimer of transmembrane domains (TMDs), a dimer of nucleotide-binding domains (NBDs), and a regulatory (R-) domain. ATP binding between the NBDs putatively induces NBD dimerization. This dimerization, in turn, is coupled to channel gating via NBD interactions with the intracellular loops of the TMDs. Disruption of local domain structure and alterations in the appropriate domain-domain assembly are associated with multiple disease-causing CFTR mutations (1-3).

Structures of mammalian and bacterial ABC transporter proteins, including the NBDs from CFTR, provide templates for understanding the biosynthetic and functional defects associated with these CF-causing mutations (3-6). Despite topological differences in the TMDs, the available ABC-transporter structures demonstrate conservation across two functional interfaces. Multiple disease-associated mutations can be mapped to both of these conserved domain-domain interaction interfaces.

The TMD-NBD interfaces are formed by intracellular loops from the TMDs and one surface of each NBD. These contacts putatively couple movements in the NBDs to conformational changes in the TMDs and regulate channel gating. Previous studies have shown that disruption of this interface leads to defects in channel biosynthesis and function $(7,8)$. Recent work has also shown that alterations to this interface can be made to promote the proper trafficking of mutant CFTR, suppressing the effects of disease-causing mutations, including $\Delta$ F508 (9).

The second interface, the NBD-NBD interface, is critical for ATP binding and hydrolysis. The ATP-binding and hydrolysis sequences are distributed across both NBDs as "half-sites." ATP binding leads to NBD dimerization and complementation of the ATP-associated sequences across the NBD dimer (10). While alterations in the NBD-NBD interface and canonical ATP-associated sequences often result in changes in channel function, biosynthetic defects have also been associated with several of these sites $(8,11)$.

Using multiple approaches to evaluate these domaindomain interactions, we are refining our understanding of the physical basis of these interactions. Computational, biochemical and cell biological approaches have been employed to probe structural and functional constraints of the TMD-NBD and NBD-NBD interface. Both interfaces show limited plasticity; however, specific alterations within both interfaces have positive impacts on domain-domain interactions.

Multiple human ABC-transporters show altered biosynthesis and trafficking as a result of mutations within the TMD-NBD interface. The sensitivity of these systems to substitution at the TMD-NBD interface demonstrates potential commonality in the biosynthetic pathways of this protein family. Additionally, profiling disease-causing mutations within the intracellular loops of human ABCtransporters and tolerated versus non-tolerated substitutions provides insight into the physico-chemical requirements for maintenance of the TMD-NBD interaction interface.

The ability to positively influence protein trafficking and function suggests that small molecule targeting to these interfaces may serve in the development of corrector and potentiator compounds.

Acknowledgements: This work was funded by the NIH and CFF.

References: 1. Du K, Sharma M, Lukacs GL. The DeltaF508 cystic fibrosis mutation impairs domaindomain interactions and arrests post-translational folding of CFTR. Nat Struct Mol Biol. 2005;12:17-25. 
2. Qu BH, Thomas PJ. Alteration of the cystic fibrosis transmembrane conductance regulator folding pathway. J Biol Chem. 1996;271:7261-4.

3. Thibodeau PH, Brautigam CA, Machius M, et al. Side chain and backbone contributions of Phe508 to CFTR folding. Nat Struct Mol Biol. 2005;12:10-6.

4. Dawson RJ, Locher KP. Structure of a bacterial multidrug ABC transporter. Nature. 2006;443:180-5.

5. Lewis HA. Structures of NBD1 deltaF508 with solubilizing mutations. PDB, 2007.

6. Lewis HA, Buchanan SG, Burley SK, et al. Structure of nucleotide-binding domain 1 of the cystic fibrosis transmembrane conductance regulator. Embo J. 2004;23:282-93.

7. Cui L, Aleksandrov L, Chang XB, et al. Domain interdependence in the biosynthetic assembly of CFTR. J Mol Biol. 2007;365:981-94.
8. Seibert FS, Linsdell P, Loo TW, et al. Disease-associated mutations in the fourth cytoplasmic loop of cystic fibrosis transmembrane conductance regulator compromise biosynthetic processing and chloride channel activity. J Biol Chem. 1996;271:15139-45.

9. Thibodeau PH, Richardson JM 3rd, Wang W, et al. The cystic fibrosis-causing mutation deltaF508 affects multiple steps in cystic fibrosis transmembrane conductance regulator biogenesis. J Biol Chem. 2010;285:35825-35.

10. Vergani P, Lockless SW, Nairn AC, et al. CFTR channel opening by ATP-driven tight dimerization of its nucleotide-binding domains. Nature. 2005;433:876-80.

11. Gregory RJ, Rich DP, Cheng SH, et al., Maturation and function of cystic fibrosis transmembrane conductance regulator variants bearing mutations in putative nucleotidebinding domains 1 and 2. Mol Cell Biol. 1991;11:3886-93.

\title{
S4.3 COTRANSLATIONAL ASPECTS OF NBD1 FOLDING AND MISFOLDING
}

\author{
William R. Skach, M.D. ${ }^{1}$ and Ineke Braakman, Ph.D. ${ }^{2}$ \\ 1. Biochemistry \& Molecular Biology, Oregon Health \& Sciences Univ., Portland, OR, USA; 2. Bijvoet Ctr. for \\ Biomolecular Research, Dept. of Chemistry, Utrecht Univ., Utrecht, Netherlands
}

CFTR misfolding and subsequent degradation constitute a primary defect in most patients with cystic fibrosis (1). Therefore, an important goal in developing novel $\mathrm{CF}$ therapies is to better understand how CFTR acquires and maintains its folded state in the cellular environment. In particular, attention has focused on NBD1 where the $\Delta$ F508 mutation confers a temperature-sensitive phenotype, decreases thermal stability, interferes with interactions between NBD1 and the membrane spanning domains, and changes NBD2 (2-5). WT NBD1 exhibits a melting temperature near $37^{\circ} \mathrm{C}$ which is reduced by loss of Phe508, resulting in an unstable form of the mutant protein. Moreover, refolding of NBD1 in vitro is very inefficient, suggesting that proper NBD1 structure is dependent upon the specific folding pathway. Because it takes 5-7 minutes to synthesize full length CFTR protein in cells, whereas secondary protein structure forms on a millisecond time scale, many aspects of folding, i.e., membrane insertion (6) and initial domain folding (7) occur cotranslationally as nascent CFTR emerges from the ribosome. This initial domain folding is followed by slow domain assembly (8) and release from a complex set of chaperone machinery (9), as confirmed by the timing of proteolytic changes in the various CFTR domains and its relatively long residence time (30-120 $\mathrm{min})$ in the ER.

Recent studies using limited proteolysis and fluorescence resonance energy transfer (FRET) have begun to tease out early folding steps that give rise to NBD1 struc- ture. A primary defect in $\triangle F 508$ CFTR arises in NBD1 and can be identified by characteristic proteolytic patterns for the recombinant, in vitro translated, and cell expressed NBD1 domain, as well as full-length CFTR. This basic defect can be rescued by a secondary suppressor mutation, I539T, which like Phe508 is located in the $\alpha$-subdomain region of NBD1 (residues 495-564). A fascinating feature of this suppressor mutation is that mice already carry a Thr residue at this position, which likely explains increased NBD1 WT and $\Delta$ F508 thermal stability compared to human NBD1, and perhaps their milder lung disease phenotype. Consistent with the above-mentioned results, I539T fully rescues $\alpha$-subdomain stability and conformation (10). Examination of ribosome-bound nascent chains by FRET has further revealed that NBD1 folding begins cotranslationally with rapid collapse of a short N-terminal subdomain (residues 389- 500) that forms a minimal ATP-binding site (11). This region contains the three-stranded ABC $\beta$-subdomain, the unstructured regulatory insertion (RI), as well as regions previously ascribed to the F1-type ATPase core. The CF-causing mutation L441P interferes with N-terminal subdomain folding and disrupts CFTR trafficking, whereas removal of the regulatory insertion, which improves trafficking of the full-length protein (12), also improves N-terminus folding efficiency. Thus efficient folding of the N-terminal subdomain appears to be a key event that may provide a template upon which the subsequent $\alpha$-subdomain and the $\alpha / \beta$ core assemble. Impor- 
tantly, kinetic analysis of N-terminal subdomain folding, as well as analysis of regions C-terminal to this subdomain, indicates that $\Delta \mathrm{F} 508$ specifically impacts subsequent folding of the $\alpha$-subdomain and/or the $\alpha / \beta$-sheet core.

References: 1. Mendoza J, Thomas P. Building an understanding of cystic fibrosis on the foundation of ABC transporter structures. J Bioenerg Biomembr. 2007;39:499-505.

2. He L, Aleskandrov L, Cui L, et al. Restoration of domain folding and interdomain assembly by second-site suppressors of the deltaF508 mutation in CFTR. FASEB J. 2010;24:3103-12.

3. Thibodeau P, Richardson J, Wang W, et al. The cystic fibrosis-causing mutation $\Delta F 508$ affects multiple steps in cystic fibrosis transmembrane conductance regulator biogenesis. J Biol Chem. 2010;285:35825-35.

4. Wang C, Protasevich I, Yang Z, et al. Integrated biophysical studies implicate partial unfolding of NBD1 of CFTR in the molecular pathogenesis of F508del cystic fibrosis. Protein Sci. 2010;10:1932-47.

5. Serohijos A, Hegedus T, Aleksandrov A, et al. Phenylalanine-508 mediates a cytoplasmic-membrane domain contact in the CFTR 3D structure crucial to assembly and channel function. Proc Natl Acad Sci USA. 2008;105:32256-61.
6. Carveth K, Buck T, Anthony V, et al. Cooperativity and flexibility of cytsic fibrosis transmembrane conductance regulator transmembrane segments participate in membrane localization of a charged residue. $\mathrm{J}$ Biol Chem. 2002;277:39507-14.

7. Kleizen B, Vlijmen T, de Jonge H, et al. Folding of CFTR is predominantly cotranslational. Mol Cell. 2005;20:277-87.

8. Du K, Sharma M, Lukacs G. The $\Delta$ F508 cystic fibrosis mutation impairs domain-domain interactions and arrests post-translational folding of CFTR. Nature Struct Mol Biol. 2004;12:17-25.

9. Wang X, Venable J, LaPointe P, et al. Hsp90 cochaperone Aha1 rescue of the human misfolding disease cystic fibrosis. Cell. 2006;127:803-15.

10. Hoelen H, Kleizen B, Schmidt A, et al. The primary folding defect and rescue of $\triangle F 508$ CFTR emerge during translation of the mutant domain. PLoS One 2010;11:e15458.

11. Khushoo A, Yang Z, Johnson A, et al. Ligand-driven vectorial folding of ribosome-bound human CFTR NBD1. Mol Cell. 2011;41:682-92.

12. Aleksandrov A, Kota P, Aleksandrov L, et al. Regulatory insertion removal restores maturation, stability and function of DeltaF508 CFTR. J Mol Biol. 2010;401:194-210.

\title{
S4.4 \\ ATP-FREE CFTR CHANNEL GATING: CONSTITUTIVE MUTANTS, R DOMAIN REGULATION AND CF RELEVANCE
}

\author{
Kevin L. Kirk, Ph.D. \\ Dept. of Physiology \& Biophysics, Univ. of Alabama, Birmingham, AL, USA
}

CFTR has two fascinating distinctions. First, it is the only member of the ABC transporter superfamily that is known to function as an ion channel. Other ABC transporters are pumps that use the free energy from ATP hydrolysis to drive substrate transport uphill against concentration gradients (1). Anion transport through the CFTR channel is a passive process driven solely by prevailing electrochemical gradients. Second, CFTR is the only known ion channel that couples an enzymatic activity to channel gating. Channel opening is conformationally linked to ATP binding and the associated dimerization of the two nucleotide binding domains (NBDs). Channel closing is facilitated by ATP hydrolysis and the subsequent release of the hydrolysis products from one of the two ATP binding sites (2-5). The rate of channel closing in the absence of ATP hydrolysis is otherwise very low due presumably to the tightness of the NBD dimer (5). Thus, the ATPase activity of CFTR has been adapted to energize a cycle of channel gating rather than chloride transport against an electrochemical gradient.

This unique enzymatic activity of the CFTR channel begs the question-how does its gating compare to that of conventional ligand-gated channels? The gating of more typical ligand-gated channels (e.g., nicotinic acetylcholine channels) obeys allosteric principles described decades ago (6-9). The first principle of an allosteric mechanism for a typical ligand-gated channel is that ligand binding shifts the equilibrium between closed and open states but is not absolutely required for channel opening. Unliganded openings are possible (albeit with a lower probability) as are constitutive mutations that increase the frequency of unliganded openings. Such mutations often locate to the symmetry axis between the ligand binding site(s) and the channel gates (7-9). CFTR differs from conventional ligand-gated channels because it usually consumes its ligand (ATP) during the gating cycle. But, it is becoming clear that CFTR channel gating does share some important features with the gating of conventional ligand-gated channels that reversibly bind their ligands. Several groups have observed that wild type CFTR channels can open (albeit rarely) in the absence of ATP $(10,11)$. In addition, a number of constitutive mutations that enhance unliganded 
CFTR activity have been reported $(11,12)$. Our group produced several such mutations within the long cytosolic loops that connect the NBDs to the transmembrane domains (TMDs) (11). These constitutive mutations locate to the putative symmetry axis of the CFTR channel between the NBDs and the TMDs when mapped onto the available crystal structures of other ABC transporters. The constitutive mutations in the cytosolic loops also enhance ligand (ATP) sensitivity, a cardinal feature of a classic allosteric gating process (reciprocity principle). These findings support a "hybrid" gating mechanism for CFTR channels that blends the allosterism of a ligandgated channel with its unique enzymatic activity (13).

The constitutive mutations that we and others have produced can be used as tools to explore fundamental aspects of CFTR channel regulation. Two specific examples that we discuss here are: (i) defining the mechanisms by which $\mathrm{R}$ domain phosphorylation enhances CFTR channel activity; and (ii) promoting unliganded activity as a means to circumvent the defective gating of $\mathrm{CF}$ mutant channels. Regarding the first example, the main physiologic cue for CFTR channel activation in vivo is phosphorylation of its $\mathrm{R}$ domain by cyclic nucleotide dependent kinases (14). The underlying mechanisms are poorly understood although there is evidence that NBD dimerization is regulated by $\mathrm{R}$ domain phosphorylation (15). By introducing constitutive mutations into channel constructs that cannot dimerize their NBDs (e.g., an NBD2 deletion construct), we determined that $\mathrm{R}$ domain phosphorylation strongly promotes CFTR channel activity in the absence of either ATP binding or NBD dimerization (11). These and other data support a model in which the $\mathrm{R}$ domain interacts with the TMDs and/or the connecting cytosolic loops to modulate channel opening. Regarding the second example, the most prevalent CF-causing mutations (G551D and F508del) strongly disrupt ATP-dependent channel gating. Importantly, the very low activities of these CF mutant channels can be markedly improved by introducing constitutive mutations in the cytosolic loops (11). Defining how these loop mutations promote unliganded channel opening may inform new strategies for augmenting the activities of CF mutant channels.

References: 1. Higgins CF. ABC transporters: from microorganisms to man. Annu Rev Cell Biol. 1992;8:67113.

2. Carson MR, Travis SM, Welsh MJ. The two nucleotide-binding domains of cystic fibrosis transmembrane conductance regulator have distinct functions in controlling channel activity. J Biol Chem. 1995;270:1711-7.

3. Aleksandrov L, Aleksandrov AA, Chang XB, et al. The first nucleotide binding domain of cystic fibrosis transmembrane conductance regulator is a site of stable nucleotide interaction, whereas the second is a site of rapid turnover. J Biol Chem. 2002;277:15419-25.

4. Vergani P, Lockless SW, Nairn AC, et al. CFTR channel opening by ATP-driven tight dimerization of its nucleotide-binding domains. Nature. 2005;433:876-80.

5. Csanády L, Vergani P, Gadsby DC. Strict coupling between CFTR's catalytic cycle and gating of its $\mathrm{Cl}$ - ion pore revealed by distributions of open channel burst durations. Proc Natl Acad Sci USA. 2010;107:1241-6.

6. Monod DJ, Wyman J, Changeux JP. On the nature of allosteric transitions: a plausible model. J Mol Biol. 1965;12:88-118.

7. Chang Y, Weiss DS. Allosteric activation mechanism of the GABA type A receptor revealed by mutation of the conserved M2 leucine. Biophys J. 1999;77:2542-51.

8. Grosman C, Zhou M, Auerbach A. Mapping the conformational wave of acetylcholine receptor channel gating. Nature. 2000;403:773-6.

9. Purohit P, Auerbach A. Unliganded gating of acetylcholine receptor channels. Proc Natl Acad Sci USA. 2009;106:115-20.

10. Hennager DJ, Ikuma M, Hoshi T, et al. A conditional probability analysis of cystic fibrosis transmembrane conductance regulator gating indicates that ATP has multiple effects during the gating cycle. Proc Natl Acad Sci USA. 2001;98:3594-9.

11. Wang W, Wu J, Bernard K, et al. ATP-independent CFTR channel gating and allosteric modulation by phosphorylation. Proc Natl Acad Sci USA. 2010;107:3888-93.

12. Szollosi A, Vergani P, Csanády L. Involvement of F1296 and N1303 of CFTR in induced-fit conformational change in response to ATP binding at NBD2. J Gen Physiol. 2010;136:407-23.

13. Kirk KL,Wang W. A unified view of CFTR channel gating: combining the allosterism of a ligand-gated channel with the enzymatic activity of an ABC transporter. J Biol Chem. 2011;286:12813-9.

14. Cheng SH, Rich DP, Marshall J, et al. Phosphorylation of the $\mathrm{R}$ domain by cAMP-dependent protein kinase regulates the CFTR chloride channel. Cell. 1991;66:1066-72.

15. Mense M, Vergani P, White DM, et al. In vivo phosphorylation of CFTR promotes formation of a nucleotidebinding domain heterodimer. EMBO J. 2006;25:4728-39. 


\title{
HAS NEWBORN SCREENING CHANGED THE CLINICAL COURSE OF THE NEWBORN WITH CF IN THE UNITED STATES?
}

\author{
Marci K. Sontag, Ph.D. ${ }^{1}$, Hebe Quinton ${ }^{2}$, Margaret Rosenfeld, M.D., M.P.H. ${ }^{3}$ and Scott D. Sagel, M.D. ${ }^{4}$ \\ 1. Epidemiology, Colorado School of Public Health, Univ. of Colorado Denver, Aurora, CO, USA; 2. Clinical \\ Research Section, Dept. of Medicine, Dartmouth Medical School, Lebanon, NH, USA; 3. Seattle Children's Hospital \\ \& Dept. of Pediatrics, Univ. of Washington School of Medicine, Seattle, WA, USA; 4. Children's Hospital Colorado \\ \& Dept. of Pediatrics, Univ. of Colorado Denver, Aurora, CO, USA
}

Newborn screening for cystic fibrosis (CF) began in the United States in the 1980s in Colorado and Wisconsin, with a handful of other states following in the subsequent two decades (1-3). Following recommendations from the CDC in late 2004 (4), and with the support of the US Cystic Fibrosis Foundation, states throughout the United States began implementing CF newborn screening programs. By December 2009, babies in all 50 states and the District of Columbia were being screened for CF. Prior to implementation of newborn screening, babies would typically present with failure to thrive, steatorrhea, respiratory symptoms, or other complications of untreated CF. While some infants with CF diagnosed by newborn screening still present with symptoms, and $15-20 \%$ of infants are still presenting with meconium ileus, US CF clinics are seeing a different type of infant with CF than in previous decades. New guidelines provide treatment and monitoring recommendations for the care of infants, with a focus on infants identified early through newborn screening $(5,6)$; however, the US CF community is still gaining an understanding of the new face of the infant with $\mathrm{CF}$.

Every year, there are approximately 1,000 new CF diagnoses in the United States reported to the US CFF Patient Registry (7). The proportion of new diagnoses made by newborn screening grew from 5-10\% in 19962001 to almost $45 \%$ in 2009 (420/960), demonstrating an annual increase in individuals diagnosed by newborn screening. The median age of diagnosis in the 1990s and early 2000s was approximately six months of age in the United States (8). Current US CFF data show a decrease in the median age at diagnosis to less than two months of age in recent years. Almost $62 \%$ of all children under the age of 2 followed at US CF centers in 2009 were diagnosed by newborn screening, and an additional $18 \%$ were identified by meconium ileus (7). Over $80 \%$ of children who are followed at US CF care centers under the age of two were identified with an early diagnosis.

Many reports have described the differences in the early course of CF between children diagnosed conventionally after the development of symptoms and those identified by newborn screening, primarily focusing on the benefits of early diagnosis on long term outcomes (9-11) or on the severe complications of a late diagnosis $(12,13)$. An analysis of the US 2000-2002 CFF Patient Registry demonstrated that infants diagnosed with symptoms had a higher preva- lence of complications throughout childhood compared to those diagnosed by newborn screening. The prevalence of stunting and wasting in the year of diagnosis, defined as height or weight for age less than the 3rd percentile, respectively was reported at 3 times the rate in symptomatically diagnosed infants (stunting 213/810, 26\%; wasting 265/815, $33 \%$ ), compared to newborn screened infants (stunting 21/239, 9\%; wasting 26/240, 11\%). Pseudomonas aeruginosa was identified in the year of diagnosis at almost twice the rate in symptomatic infants $(228 / 774,29 \%)$ compared to newborn screened infants $(32 / 211,15 \%$, ref 14$)$.

Fewer complications in early childhood are reported to the US CF Registry since nationwide implementation of CF newborn screening. In infants without meconium ileus, the annual prevalence of stunting in children diagnosed in the first two years of life declined steadily from 6-10\% in the era before widespread implementation of newborn screening (1996-2003) to 2-4\% in 2007-2009 (7). Wasting in the first two years of life declined steadily in the years between 1996 and 2009, from a high of $15 \%$ in 1996 to approximately $3 \%$ in 2009. The cumulative incidence of $P$. aeruginosa infection in the first two years of life decreased from $30 \%$ to $23 \%$ between 2002 and 2009. While other complications including hypoelectrolytemic dehydration, bleeding and hemorrhage related to vitamin $\mathrm{K}$ deficiency are not closely tracked by the US CFF, we believe that these complications are being seen less frequently because of earlier diagnosis and initiation of treatment.

Infants identified by newborn screening are presenting earlier and appear healthier than their counterparts in the era before newborn screening was widely implemented. Early bacterial infections and growth outcomes have improved dramatically since the introduction of screening. Many of these differences are due to the impact of earlier diagnosis and the initiation of aggressive care concurrent with the introduction of screening. However, the many factors that contribute to poor growth have not been defined and persist despite pancreatic enzyme supplementation. Although published guidelines for the clinical management of infants with cystic fibrosis exist (5), there is a scarcity of evidence to dictate care. New therapeutic trials to treat early $P$. aeruginosa in infants, and new initiatives to improve growth and nutrition are positively impacting the course of early $\mathrm{CF}$ in concert with newborn 
screening, yet we are in need of more studies and interventional trials in infants with $\mathrm{CF}$ to optimize outcomes in newborn screened infants. Newborn screening provides the opportunity for earlier surveillance and initiation of treatment prior to irreversible damage occurring, further changing the face of the newborn with CF.

References: 1. Hammond KB, Abman SH, Sokol RJ, et al. Efficacy of statewide neonatal screening for cystic fibrosis by assay of trypsinogen concentrations. N Engl J Med. 1991;325:769-74.

2. Farrell PM, Kosorok MR, Rock MJ, et al. Early diagnosis of cystic fibrosis through neonatal screening prevents severe malnutrition and improves long-term growth. Pediatrics. 2001;107:1-13.

3. Comeau AM, Parad RB, Dorkin HL, et al. Population-based newborn screening for genetic disorders when multiple mutation DNA testing is incorporated: a cystic fibrosis newborn screening model demonstrating increased sensitivity but more carrier detections. Pediatrics. 2004;113:1573-81.

4. Grosse SD, Boyle CA, Botkin JR, et al. Newborn screening for cystic fibrosis: evaluation of benefits and risks and recommendations for state newborn screening programs. MMWR Recomm Rep 2004;53(RR-13):1-36.

5. Borowitz D, Robinson KA, Rosenfeld M, et al. Cystic Fibrosis Foundation evidence-based guidelines for management of infants with cystic fibrosis. J Pediatr. 2009;155(6 Suppl):S73-S93.

6. Sermet-Gaudelus I, Mayell SJ, Southern KW. Guidelines on the early management of infants diag- nosed with cystic fibrosis following newborn screening. J Cyst Fibros. 2010;9:323-9.

7. Quinton HB. Dartmouth Hitchcock Medical Center. Personal Communication.

8. Cystic Fibrosis Foundation Patient Registry, 2007 Annual Data Report to the Center Directors. Bethesda, Maryland: Cystic Fibrosis Foundation, 2008.

9. Farrell PM, Kosorok MR, Laxova A, et al. Nutritional benefits of neonatal screening for cystic fibrosis. Wisconsin Cystic Fibrosis Neonatal Screening Study Group. N Engl J Med. 1997;337:963-9.

10. Farrell PM, Li Z, Kosorok MR, et al. Bronchopulmonary disease in children with cystic fibrosis after early or delayed diagnosis. Am J Respir Crit Care Med. 2003;168:1100-8.

11. Lai HJ, Cheng Y, Farrell PM. The survival advantage of patients with cystic fibrosis diagnosed through neonatal screening: evidence from the United States Cystic Fibrosis Foundation registry data. J Pediatr. 2005;147(3 Suppl):S57-S63.

12. Wilfond BS, Farrell PM, Laxova A, et al. Severe hemolytic anemia associated with vitamin $\mathrm{E}$ deficiency in infants with cystic fibrosis. Implications for neonatal screening. Clin Pediatr (Phila). 1994;33:2-7.

13. Phillips RJ, Crock CM, Dillon MJ, et al. Cystic fibrosis presenting as kwashiorkor with florid skin rash. Arch Dis Child. 1993;69:446-8.

14. Accurso FJ, Sontag MK, Wagener JS. Complications associated with symptomatic diagnosis in infants with cystic fibrosis. J Pediatr. 2005;147(3 Suppl):S37-S41.

\title{
S5.2 \\ EARLY CF LUNG DISEASE - NO TIME TO WASTE
}

\author{
Stephen M. Stick, Ph.D., MB BChir \\ 1. Respiratory Medicine, Princess Margaret Hospital for Children, Perth, WA, Australia; 2. School of Paediatrics \& \\ Child Health, Univ. of Western Australia, Perth, WA, Australia
}

Although survival has improved for successive cohorts since the early 1960 s data from British and US data registries confirm that whilst the median age of survival has improved, the mortality rate for adult sufferers has remained virtually unchanged for 4 decades. The failure to impact on adult mortality appears at odds with data that indicate a majority of individuals with CF have apparently normal lung function in mid-childhood. However, US data indicate that, consistent with mortality rates, the best modern treatment has failed to significantly alter the trajectory of decline in lung function in adults. One plausible explanation for this conundrum is that commonly used measurements of lung function, such as forced expiratory volume in one second (FEV1), underestimate the presence and progression of lung disease. Evidence supporting this assertion comes the Netherlands. De Jong et al. compared changes in FEV1 with assessments of structurual lung disease obtained using computed tomography (CT) using a validated scoring system (1). This group observed that despite stable lung function, CT scores worsened over time. de Jong and colleagues also observed structural changes in infants and young children with CF using CT. Therefore, currently available data suggest that structural changes detected by CT could be useful for detecting early manifestations of CF lung disease and for describing disease heterogeneity. Based on these observations, the Australian Respiratory Early Surveillance Team for Cystic Fibrosis (AREST CF) collaboration combined CT together with bronchoalveolar lavage (BAL) and measurements of infant and preschool lung function in an early surveillance program for children diagnosed with $\mathrm{CF}$ following newborn screening (NBS). This innovative surveil- 
lance program has provided the first comprehensive snapshot of the earliest pathophysiological changes in the lung associated with $\mathrm{CF}$ and the significant factors that contribute to structural lung disease, notably bronchiectasis.

Our first cross-sectional analyses have established that structural lung disease including bronchiectasis occurs soon after birth, is common in the first years of life and is associated with inflammation and infection (2, 3 ). Limited (3-slice) CT and BAL were performed in children 2mo-6 yr with CF diagnosed following NBS. Scans were analyzed for the presence and extent of abnormalities. The prevalence of bronchiectasis was $22 \%$ and increased with age $(\mathrm{P}=0.001)$. Factors associated with bronchiectasis included absolute neutrophil count $(\mathrm{P}=0.03)$, neutrophil elastase concentration $(\mathrm{P}=$ $0.001)$, and $P$. aeruginosa infection $(\mathrm{P}=0.03)$. Bronchial dilatation was detected in nearly $20 \%$ of children between 2 and 5 months of age and overall nearly $80 \%$ of children at this age have evidence of pulmonary disease manifest by infection, inflammation or radiological changes (2).

Early lung function data using the raised volume rapid thoraco-abdominal compression (RVRTC) technique have also demonstrated that the mean $\operatorname{FEV}(0.5) \mathrm{z}$ score did not differ between infants with $\mathrm{CF}$ and healthy control subjects less than 6 months of age $(-0.06$ and 0.02 , respectively; $\mathrm{P}=0.87)$. However, the mean $\operatorname{FEV}(0.5) \mathrm{z}$ score was lower by 1.15 in infants with CF who were older than 6 months of age compared with healthy infants $(\mathrm{P}<0.001)$ (4). FVC and FEF(75) followed a similar pattern. These data suggest that lung function, measured by forced expiration, is normal in infants with $\mathrm{CF}$ at the time of diagnosis by newborn screening but is diminished in older infants suggesting the optimal timing of therapeutic interventions aimed at preserving lung function may be within the first 6 months of life.

We are now in a position to report the first longitudinal data from the AREST CF populations.

We examined 301 paired 3-slice CT scans obtained 1 year apart in children whose first scan was at the age 1-3 years. Bronchiectasis was never present in 74 pairs $(25 \%)$, and was detected at either the initial or subsequent scan in 227 pairs $(75 \%)$. Bronchiectasis was detected at the initial scan in 133 scan pairs $(44 \%)$ and persisted at the subsequent scan in 98 pairs (74\%). Airtrapping present at the initial scan persisted in over $80 \%$ of subsequent scans. The extent of bronchiectasis increased over 1 year in $63 \%$ of scans, and air trapping in $47 \%$. Radiological progression of bronchiectasis and air trapping was associated with severe CFTR genotype, pulmonary infection and worsening neutrophilic inflammation.

We have recently reported data from 37 infants who had at least two repeat lung function measurements (5). Mean (SD) Z-scores for FVC were -0.8 (1.0), -0.9 (1.1) and -1.7 (1.2) when measured at the first visit, one-year or two-year visits respectively. Mean (SD) z-scores for FEV0.5 were -1.4 (1.2), -2.4 (1.1) and -4.3 (1.6) respectively. In those infants in whom free neutrophil elastase was detected FVC $\mathrm{z}$-scores were 0.81 lower $(\mathrm{p}=0.003)$, and FEV0.5 z-scores 0.96 lower $(\mathrm{p}=0.001)$ respectively. Significantly greater decline in FEV0.5 z-scores occurred in those infected with $S$. aureus $(\mathrm{p}=0.018)$ or $P$. aeruginosa $(\mathrm{p}=0.021)$. Pseudomonas aeruginosa infection in children less than 5 years detected by bronchoalveolar lavage can be eradicated in more than $85 \%$ of cases using standard antibiotic regimes indicating the importance of early detection of endobronchial infection (6).

We argue that intervening at diagnosis to prevent irreversible lung damage in childhood will reduce adult mortality and that outcome measures need to be defined that reflect the onset, progression and heterogeneity of lung disease to enable clinical trials with potential diseasemodifying agents (7).

References: 1. de Jong PA, Lindblad A, Rubin L, et al. Progression of lung disease on computed tomography and pulmonary function tests in children and adults with cystic fibrosis. Thorax. 2006;61:80-5.

2. Sly PD, Brennan S, Gangell C, et al. Lung disease at diagnosis in infants with cystic fibrosis detected by newborn screening. Am J Respir Crit Care Med. 2009; 180:146-52.

3. Stick SM, Brennan S, Murray C, et al. Bronchiectasis in infants and preschool children diagnosed with cystic fibrosis after newborn screening. J Pediatr. 2009;155:623-8.e1.

4. Linnane BM, Hall GL, Nolan G, et al. Lung function in infants with cystic fibrosis diagnosed by newborn screening. Am J Resp and Crit Care Med. 2008;178:1238-44.

5. Pillarisetti N, Williamson E, Linnane B, et al. Infection, inflammation and lung function decline in infants with cystic fibrosis. Am J Respir Crit Care Med. 2011;184:75-81.

6. Douglas TA, Brennan S, Gard S, et al. Acquisition and eradication of $P$. aeruginosa in young children with cystic fibrosis. Eur Respir J. 2009;33:305-11.

7. Sly PD, Ware RS, de Klerk N, et al. Randomised controlled trials in cystic fibrosis: what, when and how? Eur Respir J. 2011;37:991-3. 


\title{
S5.3 \\ DIAGNOSTIC CHALLENGES IN A SCREENED POPULATION: THE CALIFORNIA CF NEWBORN SCREENING PROGRAM EXPERIENCE
}

\author{
Carlos Milla, M.D. \\ Pediatrics, Stanford University, Palo Alto, CA, USA
}

In California, newborn screening (NBS) for cystic fibrosis started in July 2007. The development of CF NBS in California presented the challenge of assessing in a large geographic area a diverse population where ethnic minorities have a higher representation than in other parts of the United States (1). The California NBS algorithm is a three-step process based on the determination of IRT as a first step. Specimens with an elevated IRT are sent to Stanford University Molecular Genetics Laboratory for CFTR mutation analysis (Asuragen Signature ${ }^{\circledR}$ CF 2.0 ASR) with a panel of 40 mutations determined to occur with high frequency in California $(2,3,4)$. A unique feature of the California NBS algorithm is that specimens with only one mutation identified undergo further testing by temporal temperature gradient gel electrophoresis (TTGE) and focused sequencing (Ambry Genetics Aliso Viejo, CA from July 2007 to July 2009; Stanford University Molecular Genetics Laboratory from 2009 and onward). This methodology is not constrained to the detection of known mutations, as in many commercially available panels. Thus, it can detect previously known as well as novel CFTR mutations and variants (4). Only infants whose specimens have at least two mutations or variations identified are considered positive to screening and referred to accredited CF Centers (CFCs) in California for confirmatory testing through sweat chloride determination. From the time of implementation 4 years ago, over 2 million babies have been screened and the incidence of screen positives has been approximately 1 in 4,200.

The application of more sophisticated genetic based assays to newborn screening algorithms results in an increased identification of infants with mutated CFTR alleles that result in less severely affected organ function. Although more than 1,800 variants in the CFTR gene have been identified to date, for many of these variants their pathogenic potential is plainly unknown because clinical data is sparse and/or they have not been functionally characterized ex vivo (5). In addition, among the California screen-positive newborns, novel variants have been identified in $4 \%$ of those with only one mutation identified by the California-40 panel.

Specific mutations and variants associated with normal sweat electrolyte levels and mild phenotypic manifestations have been described $(6,7)$. Distinguishing sweat test-negative $\mathrm{CF}$ patients from just carriers of CFTR mutations is important for the early recognition of complications in those with the disease and for the provision of genetic counseling to carriers. Infants who are positive to the screen are referred to CF Foundation-accredited CF care centers for sweat testing and diagnostic follow up. Those with elevated sweat chloride test values are conclusively diagnosed with $\mathrm{CF}$ and followed up using current $\mathrm{CF}$ infant care guidelines. Those with lower values are clinically evaluated using follow up guidelines developed by consensus of the California CF Consortium of CF centers in partnership with the California Department of Public Health Genetic Diseases Branch (8). This includes guidelines for preparation of infants for sweat testing, testing for fecal elastase levels, close monitoring of growth parameters and respiratory microbiology (oropharyngeal, nasopharyngeal or bronchoalveolar lavage depending on the clinical circumstances). Further, guidelines for repeat sweat chloride testing and result interpretation are in place.

Given that newborns identified by NBS are usually asymptomatic, we have embarked on a longitudinal project to monitor these infants for the development of any disease manifestations. Not surprisingly, only infants with severe mutations had sweat chloride values in the diagnostic range. The remainder had values at presentation below the indeterminate cut off $(<30 \mathrm{mmol} / \mathrm{L})$. Of importance, a significant proportion of these infants (25\%) have developed clinical problems that have led to a diagnosis of $\mathrm{CF}$ based on the presence of mutations plus clinical manifestations, and this despite their low sweat chloride values (9). Although CFTR genotype and sweat chloride are correlated, neither measure is a perfect predictor of the development of clinically apparent $\mathrm{CF}$ disease. Ruling out a diagnosis of CF should not be based solely on sweat chloride values. Our experience so far emphasizes the importance of longitudinal follow up at CF Centers regardless of initial sweat chloride results. The information being generated regarding the clinical and prognostic implications of a genetic diagnosis of $\mathrm{CF}$ based on the detection of alleles with variants and novel mutations will be of great importance to further inform the natural history of the disease in this genetically diverse group.

References: 1. U.S. Census Bureau. Population estimates and national characteristics. http://www.census.gov/popest/national/asrh/ (accessed June 1, 2011).

2. Schrijver I, Ramalingam S, Sankaran R, et al. Diagnostic testing by CFTR gene mutation analysis in a large group of Hispanics: novel mutations and assessment of a 
population-specific mutation spectrum. J Mol Diagn. 2005;7:289-99.

3. Alper OM, Wong LJ, Young S, et al. Identification of novel and rare mutations in California Hispanic and African American cystic fibrosis patients. Hum Mutat. 2004;24:353.

4. Kammesheidt A, Kharrazi M, Graham S, et al. Comprehensive genetic analysis of the cystic fibrosis transmembrane conductance regulator from dried blood specimens-implications for newborn screening. Genet Med. 2006;8:557-62.

5. Castellani C, Cuppens H, Macek M Jr, et al. Consensus on the use and interpretation of cystic fibrosis mutation analysis in clinical practice. J Cyst Fibros. 2008;7:179-96.
6. Stewart B, Zabner J, Shuber AP, et al. Normal sweat chloride values do not exclude the diagnosis of cystic fibrosis. Am J Respir Crit Care Med. 1995;151:899-903.

7. Huff DS, Huang NN, Arey JB. Atypical cystic fibrosis of the pancreas with normal levels of sweat chloride and minimal pancreatic lesions. J Pediatr. 1979;94:237-9.

8. Anonymous. Minimum Guidelines for the Followup of Newborns with Positive Cystic Fibrosis Newborn Screening

Results. http://www.cdph.ca.gov/programs/nbs/Pages/default.aspx

9. Zirbes J, Koepke R, Kharrazi M, et al. Sweat chloride (SC) concentration and CFTR mutation class among infants identified by newborn screening (NBS) in California (CA). J Cyst Fibros. 2010;9(Suppl 1):S7.

\title{
S5.4 \\ UPDATE OF CFTR2: HOW GENETIC TESTING CAN IMPACT OUR CF SCREENING ALGORITHMS
}

\author{
Patrick R. Sosnay, M.D. and Garry R. Cutting, M.D. \\ Institute of Genetic Medicine, The Johns Hopkins Medical Institutions, Baltimore, MD, USA
}

The diagnostic criteria for cystic fibrosis (CF) necessitates that evidence of CFTR dysfunction must be present for a diagnosis to be made (1). That criterion can be satisfied by identification of two known CF-causing mutations. The list of mutations included in the consensus statement on diagnosis was drawn from the 23 mutations identified by the American College of Medical Genetics (ACMG) as having direct or empirical evidence of sufficient loss of CFTR function to cause disease (when paired with another CF-causing mutation) (2). However many more mutations in the CFTR gene than these 23 with well characterized disease liability have been identified (3). The Clinical and Functional Translation of CFTR (CFTR2) project was funded by the U.S. CF Foundation to address this gap in knowledge between well characterized (usually the most commonly seen) mutations, and the less commonly seen mutations with unknown disease liability. CFTR2 has compiled patient information on nearly 40,000 CF patients from North America and Europe to identify all mutations that are seen in CF patients, and then to prioritize those mutations for functional analysis.

One hundred sixty mutations are seen in nine or more patients in CFTR2, which represents an allele frequency greater than $0.01 \%$, and, in total, account for $97 \%$ of the identified alleles. Nonsense mutations $(n=35)$, mutations of the canonical nucleotides of the splice donor/acceptor sites $(n=15)$, and insertion/deletion mutations that cause a frameshift $(n=30)$ account for $50 \%$ of these 160 mutations. These classes of mutations would be expected to introduce a premature termination codon and lead to nonsense mediated RNA decay and no protein product. The clinical characteristics of patients who carry these types of mutations are all consistent with $\mathrm{CF}$, with sweat chloride concentrations clearly in the diagnostic range and with high rates of pancreatic insufficiency. The remaining 80 mutations cannot be declared deleterious on the basis of their mutation type alone, and therefore require further functional assessment. Missense changes represent a particular diagnostic challenge as they can result in a protein that is produced in inadequate quantities by the cell, one that does not conduct chloride, or a protein that is fully functional despite the amino acid substitution. The CFTR2 project in association with Vertex Pharmaceuticals coordinated the production of cell lines stably expressing the missense mutations $(n=65)$ among the top 160 for analysis of RNA and protein levels, protein maturation, and chloride conductance. Mutations predicted to alter splicing that have not been previously studied are being analyzed as well. As a result of these efforts, the number of mutations that can be rigorously characterized as CF-causing is expanded from 23 to 130 (details on www.cftr2.org). The remaining 30 mutations have varying clinical consequences as they sometimes are associated with CF, but not fully penetrant as they also may result in a single organ system CFTR-related disorder or no phenotype (4).

However, this still represents only a fraction of the total number of mutations that have been described. The CFTR2 project will continue to characterize mutations on the basis of the clinical manifestations in patients that carry them and the functional consequence of that nucleotide and/or protein change; but will never be able to totally annotate the genetic variability in CFTR. Therefore, the challenge of CFTR mutations of uncertain clinical significance and of lower penetrance must be 
considered in CF diagnosis and screening when genetic information is used in testing. The inclusion of a mutation of uncertain clinical consequence or incomplete penetrance in a newborn screening panel creates a situation (not unlike a borderline biochemical test) that leaves the family and primary care provider with unpredictable prognosis, and requires a detailed follow-up plan from the testing organization and possibly CF care center. The CFTR2 project will be able to better inform CF diagnosis, but will not eliminate all diagnostic uncertainties. It will remain up to the screening organization to balance the appropriate sensitivity and specificity for their screen with the resources available for testing and follow-up.

References: 1. Farrell PM, Rosenstein BJ, White TB, et al. Guidelines for diagnosis of cystic fibrosis in new- borns through older adults: Cystic Fibrosis Foundation consensus report. J Pediatr. 2008;153:S4-S14.

2. Watson MS, Cutting GR, Desnick RJ, et al. Cystic fibrosis population carrier screening: 2004 revision of American College of Medical Genetics mutation panel. Genet Med. 2004;6:387-91.

3. Cystic Fibrosis Mutation Database. Toronto: Cystic Fibrosis Centre at the Hospital for Sick Children. c2010. Available from: http://www.genet.sickkids.on.ca/Home.html.

4. Bombieri C, Claustres M, De BK, et al. Recommendations for the classification of diseases as CFTRrelated disorders. J Cyst Fibros. 2011;10(Supp12):S86-102.

\title{
S6.1 \\ REGULAR PHYSICAL ACTIVITY POSITIVELY INFLUENCES THE CLINICAL STATUS OF CF PATIENTS
}

\author{
Helge Hebestreit, M.D. \\ Pediatrics, University of Würzburg, Würzburg, Germany
}

Since the early 1980 s, reports of patients with cystic fibrosis $(\mathrm{CF})$ participating in competitive sports including marathon running have shown that strenuous exercise is possible despite the multi-organ disease affecting lungs, muscles, sweat glands, etc. Nowadays, most patients with $\mathrm{CF}$ engage in some endurance activities, team and individual sports, and/or strength training (1). Recent observational studies have shown that a high level of physical activity is associated with a slower decline in pulmonary function, especially in girls (2). Furthermore, physical activity correlates positively with exercise capacity in CF (3) which, on the other hand, is related to survival (4). Randomized controlled trials assessing the effects of physical training in CF have shown that regular exercise may improve physical fitness (5-10) and pulmonary function $(6-8,10,11)$ in CF. Furthermore, several studies have shown that physical conditioning can improve quality of life in patients with CF $(7,9,10)$.

Although there is some evidence suggesting that a supervised exercise intervention might be more effective than a partially unsupervised intervention over short time periods, the latter approach has definitively shown longterm benefits $(10,11)$ which may persist even after the intervention has ended (10). Although regularly repeated activities will improve performance especially in tasks similar to the trained activities, with respect to pulmonary function training mode does not seem to matter in the long run. In other words, the effects of strength training and aerobic training on pulmonary function seem to be comparable (12).

Given the available evidence in favor of an active lifestyle in patients with $\mathrm{CF}$, regular physical activity and exercise is considered important for a patient's health by most CF health professionals $(13,14)$. Exercise is well accepted among patients as well (15).

References: 1. Ruf K, Winkler B, Hebestreit A, et al. Risks associated with exercise testing and sports participation in cystic fibrosis. J Cyst Fibros. 2010;9:339-45.

2. Schneiderman-Walker J, Wilkes DL, Strug L, et al. Sex differences in habitual physical activity and lung function decline in children with cystic fibrosis. J Pediatr. 2005;147:321-6.

3. Hebestreit H, Kieser S, Rüdiger S, et al. Physical activity is independently related to aerobic capacity in cystic fibrosis. Eur Resp J. 2006;28:734-9.

4. Nixon PA, Orenstein DM, Kelsey SF, et al. The prognostic value of exercise testing in patients with cystic fibrosis. N Eng J Med. 1992;327:1785-8.

5. Orenstein DM, Franklin BA, Doerchuk CF, et al. Exercise conditioning and cardiopulmonary fitness in cystic fibrosis. The effects of a three-month supervised running program. Chest. 1981;80:392-8.

6. Kriemler S, Hebestreit A, Kieser S, et al. Six months of training improve lung functions and aerobic performance in CF (Abstract). J Cyst Fibros. 2001;(Suppl):P62.

7. Selvadurai HC, Blimkie CJ, Meyers N, et al. Randomized controlled study of in-hospital exercise training programs in children with cystic fibrosis. Pediatr Pulmonol. 2002;33:194-200.

8. Moorcroft AJ, Dodd ME, Morris J, et al. Individualized unsupervised exercise training in adults with cystic fibrosis: a 1 year randomized controlled trial. Thorax. 2004;59:1074-80. 
9. Klijn PHC, Oushoorn A, van der Ent CK, et al. Effects of anaerobic taining in children with cystic fibrosis: a randomized controled study. Chest. 2004;125:1299-305.

10. Hebestreit H, Kieser S, Junge S, et al. Long-term effects of a partially supervised conditioning programme in cystic fibrosis. Eur Respir J. 2010;35:578-83.

11. Schneiderman-Walker J, Pollock SL, Corey M, et al. A randomized controlled trial of a three year home exercise program in cystic fibrosis. J.Pediatr. 2000;136:304-10.

12. Orenstein DM, Hovell MF, Mulvihill M, et al. Strength vs. aerobic training in children with cystic fibrosis. A randomized controlled trial. Chest. 2004;126:1204-14.
13. Barker M, Hebestreit A, Gruber W, et al. Exercise testing and training in German CF centres. Pediatr Pulmonol. 2004;37:351-5.

14. Stevens D, Oades PJ, Armstrong N, et al. A survey of exercise testing and training in UK cystic fibrosis clinics. J Cyst Fibros. 2010;9:302-6.

15. Myers LB. An explorative study investigating factors associated with adherence to chest physiotherapy and exercise in adults with cystic fibrosis. J Cyst Fibros. 2009;8:425-7.

\title{
S6.2 \\ MOBILIZING MOTIVATION: BEHAVIORAL SKILL BUILDING FOR PHYSICAL ACTIVITY
}

\author{
Heather Chambliss, Ph.D. \\ Health \& Sport Sciences, University of Memphis, Memphis, TN, USA
}

Supporting behavior change can be a challenge for both health professionals and patients, yet it is the key for the long-term maintenance of physical activity and fitness. Skillbuilding strategies derived from behavioral theories can be incorporated across a variety of programs and settings.

One way to structure the physical activity counseling session is to consider an A's Approach (1):

Assess - explore current physical activity habits as well as factors that may support or hinder activity;

Advise - provide clear, structured advice considering patient needs, current behavior, self-efficacy, and physical activity guidelines;

Assist - provide behavioral assistance through behavioral skill building;

Arrange - develop a plan for monitoring short-term and long-term progress and provide feedback to support goals.

Health professionals can assist patients in meeting physical activity goals by supporting behavioral skill building. Behavioral skills which can be effective in mobilizing motivation and progress include self-monitoring, goal setting, problem solving, social support, stimulus control, and relapse prevention among others (2-4).

Self-monitoring is a skill which is consistently shown to be an important predictor of successful behavior change across health behaviors. The skill of self-monitoring involves keeping a record of current habits and future goals and can be accomplished using written logs, calendars, or technology.

Goal setting involves establishing concrete behavioral goals that meet "SMARTS" criteria. Both patients and professionals agree that goals are important; however, setting the right goal is the key.

When professionals assess factors that hinder physical activity, barriers will emerge. Problem solving, or following a stepwise approach to address barriers using the "IDEA" method, assists patients in overcoming barriers both in the current situation and in the future.

An important role of the health professional is to serve as a source of social support, providing knowledge and encouragement. Individuals should be encouraged to build a support team by identifying specific types of help needed and matching support to meet those needs.

Another behavioral skill is stimulus control, or identifying and managing triggers that prompt behaviors. Managing the environmental and psychosocial prompts that lead to healthy or unhealthy choices is a skill that helps people take control of their daily habits.

Finally, patients should be taught skills to help prevent relapse, or falling back into old behaviors. Relapse prevention teaches people how to identify high risk situations and plan ahead so that they can manage them more effectively. In addition, patients should be counseled on how to prepare for potential lapses so that they can get back on track when they do not meet their goals.

It can be helpful to consider behavioral skill building from a "toolbox" approach, so that each skill represents one tool that can be used as needed. Practitioners can help patients prepare for a physically active lifestyle by giving them the tools they need for long-term change. This type of approach can help increase motivation by establishing realistic goals, increasing confidence, and having a tailored plan of action. Thus, approaching behavior change using a skill building approach allows flexibility to tailor skills to individual needs, readiness for change, and fitness goals.

References: 1. Meriwether RA, Lee JA, Lafleur AS, et al. Physical activity counseling. Am Fam Physician. 2008;77:1129-36. 
2. Chambliss HO, King AC. Behavioral strategies to enhance physical activity participation. In: Ehrman JK, editor. ACSM's Resource Manual for Guidelines for Exercise Testing and Prescription, 6th ed, Philadelphia: Lippincott Williams \& Wilkins; 2009. p. 696-708.

3. Napolitano MA, Lewis BA, Whiteley JA, et al. Principles of health behavior change. In Ehrman JK, edi- tor. ACSM's Resource Manual for Guidelines for Exercise Testing and Prescription, 6th ed, Philadelphia: Lippincott Williams \& Wilkins; 2009. p. 710-23.

4. Whiteley JA, Lewis BA, Napolitano MA, et al. Health behavior counseling skills. In Ehrman JK, editor. ACSM's Resource Manual for Guidelines for Exercise Testing and Prescription, 6th ed, Philadelphia: Lippincott Williams \& Wilkins; 2009. p. 724-34.

\title{
S6.3 \\ ASSESSMENT OF HABITUAL PHYSICAL ACTIVITY SHOULD BE PART OF THE REGULAR ASSESSMENT OF CF PATIENTS
}

\author{
Nancy Alarie, B.Sc. P.T. \\ McGill University Health Ctr., Montreal Children's Hospital, Montreal, QC, Canada
}

There is no longer any doubt that physical activity and exercise contribute positively to the health and wellbeing of CF patients. There are several methods available to measure exercise tolerance (cardiopulmonary exercise testing, 6MWT, shuttle tests) but what about the assessment of a patient's level of activity? While the assessment of exercise tolerance is valuable and necessary it does not provide the clinician with information on the patient's day to day activities and may not be easily accessible to all clinicians. Without valid and reliable assessment tools the efficacy of our interventions becomes impossible to determine.

The World Health Organization defines physical activity as "any bodily movement produced by skeletal muscles that requires energy expenditure. The term "physical activity" should not be mistaken with "exercise." Exercise is a subcategory of physical activity that is planned, structured, repetitive, and purposeful in the sense that the improvement or maintenance of one or more components of physical fitness is the objective. Physical activity includes exercise as well as other activities which involve bodily movement and are done as part of playing, working, active transportation, house chores and recreational activities."

There are several methods available to monitor/measure habitual physical activity including: (a) doubly labelled water that measures total energy expenditure, (b) motion sensors (pedometers, accelerometers) that measure activity counts, (c) recall questionnaires and activity diaries, and more recently (d) portable physical activity monitor (SenseWear Pro 3 Armband, SWA, Body Media, Pittsburgh, USA). Each of these methods assesses a different aspect of habitual physical activity and each has definite benefits and drawbacks. The following is intended to provide an insight into the strengths and weaknesses of available tools.

The "Gold Standard" in the literature is described as direct observation. Although ideal and applicable in the research setting its use as a measure of daily patterns of activity is limited. Doubly labelled water (where the hydrogen and oxygen in water have been replaced by sta- ble isotopes) measures daily energy expenditures by calculating the elimination rates of the isotopes over time (by sampling of saliva, urine or blood). However this technique is costly and does not provide information regarding the pattern or intensity of activity.

Motion sensors detect body movements and provide an estimate of physical activity. Pedometers are small electronic devices used to estimate the mileage walked or total number of steps taken over a period of time. They are relatively inexpensive, re-useable, objective and nonreactive. On the downside they only report total counts over the observational period and cannot assess the intensity or pattern of the activities performed.

Accelerometers are more sophisticated electronic devices that measure accelerations produced by body movement. Accelerometers provide an objective, nonreactive, and re-usable tool for assessing physical activity and the newer generation of devices will be able to automatically detect patterns of activity (i.e., no need to manipulate the device). However, their ability to assess cycling, locomotion on a slope, or other activities with minimal torso movement is limited.

Subjective measures of habitual activity such as questionnaires and diaries are typically cheap, easy to administer, and often quick to perform. Only those that have been validated against more stringent measures of physical activity should be used.

The Habitual Activity Estimation Scale (HAES) has been used in several chronic illnesses. This scale has several benefits over other measures as it was designed to be used in the clinical setting, can be completed in less than 15 minutes (resulting in $100 \%$ completion rates), data can be quickly analyzed and clinicians can provide immediate feedback to patients and caregivers. Patients older than 11 years of age can complete it without assistance. The HAES has been shown to be a reliable and valid instrument for the assessment of physical activity in pediatric and adult $\mathrm{CF}$ populations.

The International Physical Activity Questionnaire (IPAQ) is a self report measure of physical activity in 
adults. "The purpose of the IPAQ is to provide a set of well-developed instruments that can be used internationally to obtain comparable estimates of physical activity. There are two versions of the questionnaire. The short version is suitable for use in national and regional surveillance systems and the long version provides more detailed information often required in research work or for evaluation purposes." Preliminary research in CF shows that it tends to underestimate physical activity in patients with lower energy expenditure activities and overestimate physical activity in patients with higher energy expenditure activities.

The Seven-Day Physical Activity Recall (7D-PAR) is an interviewer administered recall of a person's physical activity at various intensity levels over the previous 7 days. This measure is used frequently in adult studies and has demonstrated acceptable testretest reliability and validity when compared to electronic monitoring.

Preliminary studies in children have indicated adequate validity in relation to direct observation. Thus far only one reported abstract investigated the use of 7DPAR in a CF population and found that the ICC between accelerometry and 7D-PAR was not strong enough to assess individual patients.

Portable physical activity monitors, specifically the SenseWear Pro 3 have been designed to improve the measurement of physical activity. This monitor integrates a bi-axial accelerometer, galvanic skin resistance, heat flux, skin and near body temperature to estimate the energy expenditure and step count. The armband is worn on the upper right arm. This system was evaluated in a population of adult $\mathrm{CF}$ patients and the diagnosis of $\mathrm{CF}$ did not seem to impact the system's ability to accurately estimate energy expenditure. However it did tend to underestimate energy expenditure with increasing exercise intensity (consistently reported in other studied populations).

Although the ideal method for measuring physical activity remains elusive, regular assessment by the clinician is key to the management of our CF patients.
Use of physical activity questionnaires has many practical values but should be limited to those that have been assessed for validity and reliability in the CF population. Pedometers and accelerometers decrease the subjectivity of questionnaires but have limited ability to assess cycling movements and upper limb movements. Doubly labelled water and direct observation appear to be the most objective measures but their applicability in the clinical setting is non-existent. Portable activity monitors do show promise but are not without limitations.

References: 1. Wilkes DL, Schneiderman JE, Nguyen T, et al. Exercise and physical activity in children with cystic fibrosis. Pediatr Respir Rev. 2009;10:105-9.

2. Wells GD, Wilkes DL, Schneiderman-Walker JE, et al. Reliability and validity of the Habitual Activity Estimation Scale (HAES) in patients with cystic fibrosis. Pediatr Pulmonol. 2008;43:345-53.

3. Hay JA. Development of the Habitual Estimation Scale for clinical research: A systematic approach. Pediatr Exerc Sci. 2006;18:193-202.

4. Schneiderman-Walker J, Wilkes DL, Strug L, et al. Sex differences in habitual physical activity and lung function decline in children with cystic fibrosis. J Pediatr. 2005;147:321-6.

5. Shepard RJ. Limits to the measurement of habitual activity by questionnaires. $\mathrm{Br} \mathrm{J}$ Sports Med. 2003;37:197-206.

6. Dwyer TJ, Alison JA, McKeough ZJ, et al. Evaluation of the SenseWear activity monitor during exercise in cystic fibrosis and in health. Respir Med. 2009;103:1511-7.

7. Sirard JR, Pate RR. Physical activity assessments in children and adolescents. Sports Med. 2001;31:439-54.

8. Button BM, Rasekaba T, Wilson JW, et al. Validation of the International Physical Activity Questionnaire in adults with cystic fibrosis. Pediatr Pulmonol. 2010;45(S33):382.

9. Ruf K, Fehn S, Bachman M, et al. Validation of questionnaires assessing physical activity in patients with cystic fibrosis. Pediatr Pulmonol. 2010;45(S33):382.

10. http://www.who.int/topics/physical_activity/en/ 11. www.ipaq.ki.se

\title{
S6.4 \\ DEVELOPING PHYSICAL ACTIVITY TARGETS
}

\author{
Frank J. Cerny, Ph.D. \\ 1. Children's Lung Center, Women \& Children's Hospital of Buffalo, Buffalo, NY, USA; 2. Pediatrics \& Exercise \& \\ Nutrition Sciences, Univ. at Buffalo, Buffalo, NY, USA
}

It is clear that regular, intentional, physical activity (PA) should be part of a healthy lifestyle, including for patients with cystic fibrosis. There are both disease specific, e.g., improved airway clearance, improved lung function, better control of CF-related diabetes, etc., as well as more general, e.g., cardiovascular and musculoskeletal health, benefits of PA. It is equally clear that there is no "one-size-fits-all" target for PA. The following is intended to provide a general guide for PA recommendations followed by suggestions for children.

PA recommendations of any kind should be based on knowledge of the clinical status, including pulmonary function, and of the exercise response, including whether desaturation occurs and at what level of exercise. 
PA targets can be attained using a combination of both formal, e.g., exercise prescription and leisure-time activity recommendations, and informal, but no less intentional, habitual or lifestyle PA recommendations. In any case the recommendation should be based on the patient's response to three critical questions: "What kinds of activities do you like to do?," "Who is going to be your partner?" and "What sorts of things keep you from being physically active?"

Success in meeting PA targets will be improved if participants can select from a variety of favorite activities and participate in these activities on an "as desired" basis. Success is further enhanced by making a commitment with an activity partner. For children and young adults, promoting activity with best friends significantly increases time spent in moderate-to-vigorous activity while adults benefit from the accountability associated with a committed partner. Finally, PA is more easily integrated into a lifestyle with the identification of, and removal or minimization of barriers - excuses, in many cases.

Making PA a regular part of one's daily routine is difficult for most people, particularly if they are currently sedentary. It is therefore best to start the exercise recommendation with advice and encouragement as to how to become a regular exerciser. For some the first step is to find ways to incorporate activity into one's daily routine. These lifestyle changes might include changing one's commute or shopping habits to include walking or riding a bicycle for all or part of the distance or simply parking the car at the far side of the parking lot and walking, taking the stairs instead of the elevator, walking during work breaks, etc. An initial goal should be to accumulate 30 minutes of this kind of activity 5 days a week. The use of a pedometer can enhance motivation for lifestyle PA. Recognizing that less than 5,000 steps per day represents a sedentary lifestyle the initial goal should be to increase the daily step count to $>10,000$ per day over a period of time.

As the individual approaches the goal of 30 minutes, 5 days a week or 10,000 steps the recommendation should include advice on increasing exercise intensity to moderate to vigorous levels. Moderate exercise intensity should allow the person to maintain a conversation without becoming breathless while vigorous intensity would involve a sense of breathlessness. Once a person has become accustomed to an increase in habitual, lifestyle
PA it becomes easier to move to leisure-time PA or more formal exercise recommendations.

The compendium of physical activities (1-3) provides a listing of hundreds of activities and their energy cost. Energy cost is listed in terms of METS (Metabolic Equivalents, with $1 \mathrm{MET}=3 \mathrm{~mL} \mathrm{O} / \mathrm{kg} / \mathrm{min}$ ) allowing one to recommend a variety of specific PA at moderate (3-6 METs) or vigorous (>6 METs) activity intensities. For instance, encouraging activities such as general carpentry or sweeping floors or bicycling with light effort would fulfill a recommendation for moderate activity while encouraging jogging at $6 \mathrm{mph}$, shoveling snow or higher intensity bicycling would fulfill a recommendation for vigorous activity. In this way a variety of activities can be combined to attain the goal of a minimum of 30 minutes of moderate-vigorous activity 5 days a week.

Some patients will require a more formal exercise prescription to be carried out in an inpatient or outpatient rehabilitation setting; some may find that specific exercise prescriptions make it easier to attain the above-described goals outside of a rehabilitation setting. This more specific prescription approach also allows for safe recommendations for strength training activities. The prescription for aerobic exercise will use heart rate as the marker for exercise intensity and a target of $>60 \%$ of Heart Rate Reserve calculated from a peak/maximal exercise test $(\mathrm{HRR}=($ Peak HR - Rest HR $) \%$ intensity + Rest HR $)$.

The most effective way to involve children in regular activities that will meet the recommendation is to engage family and friends in an active lifestyle, including leisure activities and sports. Children can be effectively disengaged from sedentary activities by providing rewards for reducing those activities, allowing them to select their own alternatives. Similarly, connecting children with active friends is effective in increasing the amount of time spent in moderate to vigorous activity on a daily basis.

References: 1. Ainsworth, BE, Haskell, WL, Whitt, $\mathrm{MC}$, et al. Compendium of physical activities: an update of activity codes and MET intensities. Med Sci Sports Exerc. 2000;32 (Suppl):S498-504.

2. Harrell JS, McMurray RG, Baggett CD, et al. Energy costs of physical activities in children and adolescents. Med Sci Sports Exerc. 2005;37:329-336.

3. The Compendium of Physical Activities Tracking Guide. Available from: http://prevention.sph.sc.edu/ tools/docs/documents_compendium.pdf 


\title{
S7.1 \\ HOST-PATHOGEN INTERACTIONS IN STAPHYLOCOCCUS AUREUS LUNG DISEASE
}

\author{
Julie Bubeck Wardenburg, M.D., Ph.D. \\ Dept. of Pediatrics \& Microbiology, University of Chicago, Chicago, IL, USA
}

Staphylococcus aureus is one of the most significant causes of human bacterial infection, contributing to morbidity and mortality in individuals of all ages (1). The virulence properties of this microorganism facilitate infection of a wide array of tissues and organs, including the human lung. Historically, S. aureus pneumonia has been a scourge of the critical care environment, not often documented as a cause of community acquired disease. However, an alarming trend has been noted for over a decade, as an increase in the incidence of severe $S$. aureus pneumonia in otherwise healthy adults and children has been reported. Recent studies suggest that approximately 50,000 cases of $S$. aureus pneumonia occur in the U.S. alone each year, with more than half caused by methicillin-resistant $S$. aureus (MRSA) (2). Increasing antimicrobial resistance among $S$. aureus strains and the appearance of particularly virulent isolates of this pathogen within the community have rendered conventional antimicrobial therapies obsolete, resulting in increased mortality and cost of care (3). Concomitant with these changes, individuals with cystic fibrosis have experienced an increasing burden of drug-resistant S. aureus disease (4). Novel therapeutic approaches to both prevent and treat invasive pulmonary infection with $S$. aureus, and especially MRSA, are necessary. As $S$. aureus is a key colonizing organism and pathogen in both the normal and CF host, understanding the principles that govern the interaction of the host with the pathogen in both patient populations is likely to provide keen insights that will enhance our ability to combat $S$. aureus lung disease.

Studies of disease pathogenesis. As a first step in understanding the pathogenesis of $S$. aureus pneumonia, animal models have been used to define the pathogenencoded virulence factors that facilitate lung infection. Initial work in the field led to the observation that staphylococcal Protein A, a cell-wall associated protein, contributes to lung disease by virtue of its ability to interact with the tumor-necrosis factor-alpha receptor, TNFR1, and stimulate proinflammatory responses (5). Disruption of the interaction of Protein A with TNFR1, either by genetic deletion of Protein A in engineered S. aureus, or in TNFR1 knockout mice, mitigates disease (5). Subsequently, studies in an adult, immunocompetent mouse model of $S$. aureus pneumonia have revealed a profound defect in virulence when mice were infected with strains engineered to lack expression of $\alpha$-hemolysin (Hla), a pore-forming exotoxin that is produced by almost all strains of $S$. aureus (6). Further investigation of $\alpha$ hemolysin in $S$. aureus pneumonia revealed the central role of this single toxin in the modulation of virulence, correlating $\alpha$-hemolysin expression with mortality in animal studies and direct injury to the pulmonary alveolar epithelium (7). As a toxin that forms injurious pores in the eukaryotic cell membrane, the mechanism of $\alpha$ hemolysin-induced injury to the lung appears conceptually simple - the toxin can induce irreversible damage to the plasma membrane, culminating in cell death and organ dysfunction. The recent identification of A Disintegrin and Metalloprotease 10 (ADAM10) as the cellular receptor for $\alpha$-hemolysin is anticipated to yield further insights into the mechanism of toxin action, and may also permit a clearer understanding of host susceptibility to disease (8). ADAM10 plays an essential role in the maintenance and turnover of the epithelium (9). The use of this protein as a toxin receptor raises the interesting possibility that the toxin may not only bind to the receptor to gain access to the host cell, but may take advantage of the native function of ADAM10 to specifically injure the epithelium of the lung.

In addition to studies on $S$. aureus virulence factors such as Protein A and $\alpha$-hemolysin, the nature of the host immunologic response to the pathogen plays a critical role in establishing disease severity. Depletion of alveolar macrophages leads to enhanced mortality from experimental MRSA pneumonia, however does not impact on the bacterial load in the lung tissue. In contrast, depletion of host dendritic cells was associated with an increased bacterial load in the lung, but no alterations in mortality (10). The differential requirements for specific immune cell populations in governing distinct facets of disease pathogenesis further underscore the complexity of the host-pathogen interaction in S. aureus pneumonia.

Implications for prevention and therapy. Both active immunization with a non-toxigenic variant of $\alpha$ hemolysin and passive immunization with rabbit anti- $\alpha-$ hemolysin immune sera protected animals from lethal $S$. aureus pneumonia (7). Targeting the toxin in this fashion may therefore be a highly effective strategy to limit $S$. aureus lung disease. In addition, it is reasonable to propose that strategies that interfere with the ability of the toxin to bind to its host receptor, ADAM10, may also be of clinical value. Host-targeted approaches to the prevention or amelioration of disease are also essential to consider, including strategies to manipulate the host cytokine and chemokine response to infection and minimize intrapulmonary inflammation (10). At present, the field remains limited by a lack of knowledge of the hostpathogen interaction in human disease. While experimental model systems have provided keen insight into 
the complexities of this interaction, this knowledge will need to be extended to the human population through well-designed clinical studies aimed at identifying factors that contribute to host susceptibility as well as evaluate the contribution of staphylococcal virulence factors to disease. Moreover, it will be essential to understand the host-pathogen interaction in the setting of underlying $\mathrm{CF}$ as the nature of this interaction may be fundamentally different, demanding distinct approaches to the prevention and treatment of $S$. aureus lung disease.

References: 1. Lowy FD. Staphylococcus aureus infections. N Engl J Med. 1998;339:520-32.

2. Kuehnert MJ, Hill HA, Kupronis BA, et al. Methicillin-resistant-Staphylococcus aureus hospitalizations, United States. Emerg Infect Dis. 2005; 11:868-72.

3. Chambers HF. Community-associated MRSAresistance and virulence converge. N Engl J Med 2005;352:1485-7.

4. de Vrankrijker AM, Wolfs TF, van der Ent CK. Challenging and emerging pathogens in cystic fibrosis. Paediatr Respir Rev. 2010;11:246-54.
5. Gomez MI, Lee A, Reddy B, et al. Staphylococcus aureus protein A induces airway epithelial inflammatory responses by activating TNFR1. Nat Med. 2004;10:842-8.

6. Bubeck Wardenburg J, Patel RJ, Schneewind O. Surface proteins and exotoxins are required for the pathogenesis of Staphylococcus aureus pneumonia. Infect Immun. 2007;75:1040-4.

7. Bubeck Wardenburg J, Schneewind O. Vaccine protection against Staphylococcus aureus pneumonia. J Exp Med. 2008;205:287-94.

8. Wilke GA, Bubeck Wardenburg J. Role of a disintegrin and metalloprotease 10 in Staphylococcus aureus alpha-hemolysin-mediated cellular injury. Proc Natl Acad Sci USA. 2010;107:13473-8.

9. Reiss K, Saftig P. The "a disintegrin and metalloprotease" (ADAM) family of sheddases: physiological and cellular functions. Semin Cell Dev Biol. 2009;20:126-37.

10. Martin FJ, Parker D, Harfenist BS, et al. Participation of CD11c(+) leukocytes in methicillin-resistant Staphylococcus aureus clearance from the lung. Infect Immun. 2011;79:1898-904.

\title{
S7.2 \\ MOLECULAR EPIDEMIOLOGY AND THE IMPACT OF SOCIAL NETWORKING IN $S$. AUREUS TRANSMISSION
}

\author{
Lisa Saiman, M.D., M.P.H. \\ Pediatrics, Columbia University, New York, NY, USA
}

Introduction. The molecular epidemiology of bacterial pathogens can be integrated with social networking to increase our understanding of infectious disease transmission in community-based settings (1). This strategy has been used as a public health measure to study transmission of Staphylococcus aureus $(2,3)$ in the community, including closed communities such as prisons (4) or among intravenous drug users (5). Integrating molecular epidemiology with social networking strategies could potentially be used to better define transmission of $S$. aureus in $\mathrm{CF}$ and has implications for prevention.

Molecular typing strategies. Several molecular typing strategies may be used to distinguish $S$. aureus strains. These include pulse-field gel electrophoresis (PFGE), multi-locus sequence typing (MLST), S. aureus protein A typing (spa typing), and potentially microarray technology. Strategies used for community settings must be able to successfully perform, store, and compare huge amounts of sequencing data required to define unique and shared $S$. aureus strains.

Social networking strategies. Community-based and population settings are constantly changing making a total understanding of all factors involved in disease transmission impossible. Nonetheless, social network methodologies use group dynamics including social contacts, environments, and risk behaviors to provide insights into disease transmission. Three social network levels of how individuals are connected to others can be studied: the dyad, the personal network, and the community. For the dyad and personal network, direct relationships are evaluated while for the community network, both direct and indirect relationships are evaluated. There are obviously limitations to social networking studies. Such studies cannot readily observe risk behaviors, particularly those that may be deliberately hidden; links between people can be difficult to assess over long time intervals or large distances; and non-responders can have significant, but difficult to predict, influences on data.

Integrating molecular epidemiology and social network strategies. Investigators emphasize that bacterial molecular relatedness does not describe transmission dynamics without concurrent interview data that provides supporting epidemiology. Investigators further emphasize that even partial interview data can reveal links that may be brief, anonymous, or through a central individual.

Social networking techniques potentially can elucidate infectious disease transmission dynamics including the settings where transmission could have occurred which is central to developing preventive strategies. For instance, integration of data from DNA fingerprinting with social network analysis allowed identification of 
infectious TB cases and the sites of transmission (6). A similar strategy was used to explore transmission of gonorrhea (7). Another salient example of this integration occurred in a study of $S$. aureus transmission among inhalational drug users (2).

Relevance to CF. In CF, acquisition of both methicillin-susceptible $S$. aureus (MSSA) and methicillinresistant $S$. aureus (MRSA) is most likely different than acquisition of other potential pathogens. $S$. aureus, including MRSA and MSSA, can be colonizing flora of the skin (e.g., axilla), anterior nares, throat, and anogenital tract. Unlike other CF pathogens, person-to-person transmission of this microorganism can occur among family members and close contacts with and without CF (8). Several molecular epidemiology studies of MRSA in CF have demonstrated acquisition of so-called community acquired strains (8-10).

Promoting an increased understanding of the role of social networking in $\mathrm{CF}$ could increase the effectiveness of prevention efforts and sustain eradication efforts of MRSA. Integration of molecular typing and social networking is feasible in CF due to the interdisciplinary infrastructure that exists in CF care centers throughout the world.

Future Directions. Integration of molecular typing and social networking strategies within existing CF research on MRSA could facilitate our understanding of transmission and prevention. Future studies could investigate the virulence determinants that are associated with colonization and transmission of MRSA.

References: 1. Lowy FD, Miller M. New methods to investigate infectious disease transmission and pathogenesis-Staphylococcus aureus disease in drug users. Lancet Infect Dis. 2002;2:605-12.
2. Quagliarello B, Cespedes C, Miller M, et al. Strains of Staphylococcus aureus obtained from drug-use networks are closely linked. Clin Infect Dis. 2002;35:671-7.

3. Miller M, Cook HA, Furuya EY, et al. Staphylococcus aureus in the community: colonization versus infection. PLoS One. 2009;4:e6708.

4. Lowy FD, Aiello AE, Bhat M, et al. Staphylococcus aureus colonization and infection in New York State prisons. J Infect Dis. 2007;196:911-8.

5. Gwizdala RA, Miller M, Bhat M, et al. Staphylococcus aureus colonization and infection among drug users: Identification of hidden networks. Am J Public Health. 2011;101:1268-76.

6. Klovdahl AS, Graviss EA, Yaganehdoost A, et al. Networks and tuberculosis: an undetected community outbreak involving public places. Soc Sci Med. 2001;52:681-94.

7. Ward H, Ison CA, Day SE, et al. A prospective social and molecular investigation of gonococcal transmission. Lancet. 2000;356:1812-7.

8. Stone A, Quittell L, Zhou J, et al. Staphylococcus aureus nasal colonization among pediatric cystic fibrosis patients and their household contacts. Pediatr Infect Dis J. 2009:28:895-9.

9. Cafiso V, Bertuccio T, Spina D, et al. Methicillin resistance and vancomycin heteroresistance in Staphylococcus aureus in cystic fibrosis patients. Eur J Clin Microbiol Infect Dis. 2010;29:1277-85.

10. Glikman D, Siegel JD, David MZ, et al. Complex molecular epidemiology of methicillin-resistant Staphylococcus aureus isolates from children with cystic fibrosis in the era of epidemic community-associated methicillin-resistant $\mathrm{S}$ aureus. Chest. 2008;133:1381-7.

\title{
S7.3 TREATMENT PARADIGMS FOR MRSA
}

\author{
Loren Miller, M.D., M.P.H. \\ 1. Div. of Infectious Diseases, Harbor-UCLA Medical Ctr., Torrance, CA, USA; 2. David Geffen School of Medicine \\ at UCLA, Los Angeles, CA, USA
}

There are limited data on treatment of Staphylococcus aureus and methicillin resistant Staphylococcus aureus (MRSA) in patients with cystic fibrosis. Cystic fibrosis (CF)-specific $S$. aureus and MRSA treatment studies are uncommon. Much of what is known about treatment is extrapolated from experience in other populations.

Treatment of MRSA has evolved based on the emergence of infection caused by community-associated MRSA (CA-MRSA) strains. These strains are typified in the U.S. by $p v l$-containing USA300 strains. These newer strains have resulted in wider therapeutic options, as these strains are often susceptible to older antibiotics.
The emergency of small colony variants (SCV) of MRSA after MRSA treatment may further increase challenges in CF treatment.

There are many antibiotics options for MRSA treatment. Vancomycin, a glycopeptide, has been the traditional antimicrobial agent of choice for MRSA treatment. Many have argued that traditional dosing, $15 \mathrm{mg} / \mathrm{kg}$ every twelve hours, is inadequate, especially for severe infections such as bacteremia and pneumonia. Observational studies that found high vancomycin MICs $(\geq 2$ $\mathrm{mcg} / \mathrm{mL}$ ) are independently associated with poorer outcomes, specifically lower treatment response and higher 
infection-related mortality. Many recommend that serious MRSA infections require dosages that exceed traditional recommendations. Recent guidelines by the ATS/IDSA Committees recommend targeting higher vancomycin troughs of $15-20 \mathrm{mcg} / \mathrm{mL}$ in patients with health-care related MRSA pneumonia, rather than the lower targets (of 10 or less) recommended in the past.

The recent emergence of glycopeptide-intermediate and vancomycin-resistant $S$. aureus (GISA and VRSA) strains is very concerning. GISA strains have been reported to emerge in patients with $\mathrm{CF}$, typically after prolonged exposure to vancomycin. These strains may be missed by traditional microbiologic testing. There are very limited data for newer glycopeptide, telavancin, which is approved only for the treatment of skin and skin structure infections (SSTIs).

Several older antimicrobial agents have been used increasingly for the treatment of MRSA. However, data on efficacy is very limited. TMP-SMX is active in vitro against many MRSA strains. The largest published trial on the use of TMP-SMX for S. aureus found that TMPSMX demonstrated a lower clinical cure rate for $S$. aureus infection compared to vancomycin ( $85 \%$ vs. $98 \%$ ). Many experts consider it a viable treatment option for SSTIs, but data on pulmonary infections are very limited.

Clindamycin, a lincosamide, has been used successfully to treat MRSA infections, however, antimicrobial resistance is greater than $10 \%$ in some areas. Some MRSA isolates develop resistance when exposed to lincosamides and macrolides, such as erythromycin. This inducible resistance can be detected via the D-test. Most experts argue that confirming a negative D-test is critical when considering clindamycin treatment of a serious $S$. aureus infection. The ability of clindamycin to inhibit pvl expression in vitro is a theoretic advantage of using clindamycin for treatment, although the clinical benefit of this inhibition unclear.

The tetracyclines doxycycline and minocycline are active against MRSA, although their use in younger children is contraindicated. These antibiotics have successfully been used to treat MRSA infections in small case series and clinical trials, although there are extremely limited data for patients with CF. Tigecyline, a new and related drug lacks an oral formulation and there are no compelling data that this newer drug provides advantages over older tetracyclines. A newer generation cephalosporin, ceftaroline, is the only commercially available beta-lactam with activity against MRSA. It is approved for the treatment of communityacquired pneumonia, but there are no data on CF treatment.

Linezolid, an oxazildanone antimicrobial, comes in both oral and intravenous formulation, which has made it useful in patients with CF. There are retrospective data suggesting that in treatment of ventilator associated pneumonia it may have greater efficacy and lower mortality compared with vancomycin. However, its pharmacokinetics in adults and children with $\mathrm{CF}$ is controver- sial; linezolid dosing may need to be increased to 3 times per day in these patients. Additionally, linezolid-resistant MRSA have been reported in patients with CF. Furthermore, bone marrow and neurologic toxicity with prolonged linezolid treatment can be problematic.

Daptomycin, a lipopeptide with bactericidal activity against $S$. aureus, should not be used in the treatment of pneumonia as it is inactivated by pulmonary surfactant. There are data rifampin may provide additional benefit to standard therapy in the treatment of $S$. aureus, but data are inconsistent and rifampin is associated with many drug interactions. Rifampin therapy is limited by the appearance of rifampin resistance during therapy, especially when used as monotherapy.

Data on optimal MRSA decolonization and eradication are not clear and continue to emerge. Eradication of colonization of other household members may be important for eliminating colonization in the MRSA colonized patient. In CF patients, $S$. aureus biofilms in the upper airways (e.g., sinuses) and lower airways present challenges for eradication efforts. In non-CF patients, there are data suggesting that mupirocin in combination with chlorhexidine may eliminate short-term colonization and reduce skin infection with MRSA, but these regimens do little to prevent airway colonization. A recent investigation in CF patients using aggressive regimens for MRSA eradication demonstrated that regimens that typically combined 2 oral antibiotics plus nebulized vancomycin plus topical skin and nasal disinfection may be effective at eradicating MRSA colonization, with eradication rates of approximately $80 \%$. Some patients required multiple courses of therapy to achieve eradication. The clinical benefit of eradication is incompletely understood.

References: 1. Hidayat LK, Hsu DI, Quist R, et al. High-dose vancomycin therapy for methicillin-resistant Staphylococcus aureus infections: efficacy and toxicity. Arch Intern Med. 2006;166:2138-44.

2. American Thoracic Society; Infectious Diseases Society of America. Guidelines for the management of adults with hospital-acquired, ventilator-associated, and healthcare-associated pneumonia. Am J Respir Crit Care Med. 2005;171:388-416.

3. Moran GJ, Krishnadasan A, Gorwitz RJ, et al. Methicillin-resistant $S$. aureus infections among patients in the emergency department. $\mathrm{N}$ Engl J Med. 2006;355:666-74.

4. Markowitz N, Quinn EL, Saravolatz LD. Trimethoprim-sulfamethoxazole compared with vancomycin for the treatment of Staphylococcus aureus infection. Ann Intern Med. 1992;117:390-8.

5. Deresinski S. Methicillin-resistant Staphylococcus aureus: an evolutionary, epidemiologic, and therapeutic odyssey. Clin Infect Dis. 2005;40:562-73.

6. Adra M, Lawrence KR. Trimethoprim/sulfamethoxazole for treatment of severe Staphylococcus aureus infections. Ann Pharmacother. 2004;38:338-41. 
7. Frank AL, Marcinak JF, Mangat PD, et al. Clindamycin treatment of methicillin-resistant Staphylococcus aureus infections in children. Pediatr Infect Dis J. 2002;21:530-4.

8. Siberry GK, Tekle T, Carroll K, et al. Failure of clindamycin treatment of methicillin-resistant Staphylococcus aureus expressing inducible clindamycin resistance in vitro. Clin Infect Dis. 2003;37:1257-60.

9. Gorwitz RJ, Jernigan DB, Powers JH, et al. Strategies for clinical management of MRSA in the community: Summary of an experts' meeting convened by the Centers for Disease Control and Prevention. 2006. Available at: http://cdc.gov/ncidod/dhqp/pdf/ar/CAMRSA_ExpMtgStrategies.pdf. Accessed 5/22/06.

10. Micek ST, Dunne M, Kollef MH. Pleuropulmonary complications of Panton-Valentine leukocidinpositive community-acquired methicillin-resistant Staphylococcus aureus: importance of treatment with antimicrobials inhibiting exotoxin production. Chest. 2005;128:2732-8.

11. Lewis SA, Altemeier WA. Correlation of in vitro resistance of Staphylococcus aureus to tetracycline, doxycycline, and minocycline with in vivo use. Chemotherapy. 1976;22:319-23.

12. Ruhe JJ, Monson T, Bradsher RW, et al. Use of long-acting tetracyclines for methicillin-resistant Staphy- lococcus aureus infections: case series and review of the literature. Clin Infect Dis. 2005;40:1429-34.

13. Wunderink RG, Rello J, Cammarata SK, et al. Linezolid vs vancomycin: analysis of two double-blind studies of patients with methicillin-resistant Staphylococcus aureus nosocomial pneumonia. Chest. 2003;124:1789-97.

14. Kollef MH, Rello J, Cammarata SK, et al. Clinical cure and survival in Gram-positive ventilator-associated pneumonia: retrospective analysis of two double-blind studies comparing linezolid with vancomycin. Intensive Care Med. 2004;30:388-94.

15. Jung YJ, Koh Y, Hong SB, et al. Effect of vancomycin plus rifampicin in the treatment of nosocomial methicillin-resistant Staphylococcus aureus pneumonia. Crit Care Med. 2010;38:175-80.

16. Doe SJ, McSorley A, Isalska B, et al. Patient segregation and aggressive antibiotic eradication therapy can control methicillin-resistant Staphylococcus aureus at large cystic fibrosis centres. J Cyst Fibros. 2010;9:104-9.

17. Filleron A, Chiron R, Reverdy ME, et al. Staphylococcus aureus with decreased susceptibility to glycopeptides in cystic fibrosis patients. J Cyst Fibros. 2011 May 31. [Epub ahead of print]

18. Keel RA, Schaeftlein A, Kloft C, et al. Pharmacokinetics of intravenous and oral linezolid in adults with cystic fibrosis. Antimicrob Agents Chemother. 2011;55:3393-8.

\title{
S7.4 \\ CLINICAL TRIAL DESIGNS FOR METHICILLIN-RESISTANT STAPH AUREUS IN CF
}

\author{
Michael P. Boyle, M.D. ${ }^{1}$, Mark Jennings ${ }^{1}$ and Elliot C. Dasenbrook ${ }^{2}$ \\ 1. The Johns Hopkins Hospital, Baltimore, MD, USA; 2. Case Medical Center, Cleveland, OH, USA
}

Methicillin-resistant Staphylococcus aureus (MRSA) is a particularly important emerging pathogen in CF. The prevalence of infection with MRSA in the CF community has increased from $4 \%$ in 1999 to $23.7 \%$ in 2009 (1). Recently, two very large CF observational studies utilizing the CF Foundation's National Patient Registry database have suggested that infection with MRSA is associated with worse clinical outcomes. The first, published in 2008, demonstrated that persistent respiratory infection with MRSA is associated with a more rapid decline in lung function as measured by $\mathrm{FEV}_{1}$ (2). The second, published in JAMA in 2010, demonstrated an association between persistent MRSA infection and increased mortality, even after adjustment for severity of lung disease (3). Given the striking increase in both prevalence of MRSA infection in CF and evidence of a detrimental effect of MRSA on CF clinical outcomes, there has been growing interest in treatment protocols designed to treat and/or eradicate MRSA CF respiratory infection. Highlighting care- givers' concerns about MRSA, a recent survey of U.S. pediatric and adult CF Center Directors identified studies to determine appropriate treatment of MRSA as their top clinical priority.

Previous Studies of Treatment of MRSA in CF. There have been several small clinical studies in CF assessing MRSA treatment regimens and their effectiveness in eradicating MRSA from the respiratory tract. These studies have been limited however by small study populations, lack of control groups, single-center retrospective design, variable follow-up, and failure to distinguish incident vs persistent MRSA infection (4-7). Doe reported the largest experience to date with a retrospective review of 37 patients (7). Although many different eradication regimens were used, most of the patients in their cohort were treated with a combination of two oral antibiotics (rifampicin, fusidic acid or trimethoprim) and nebulized vancomycin. They reported eradication of MRSA in $81 \%$ of the participants at 6 months. There was no distinction made between those with incident MRSA 
and those with persistent MRSA infection in the study analysis, although they report that approximately $38 \%$ of those included had had multiple positive MRSA cultures. Garske focused on treatment of CF adults with persistent MRSA (4). The study enrolled seven CF adults with persistent MRSA (average FEV1 36\% predicted, all with chronic PA, 6/7 with previous IV MRSA therapy); 5 of the 7 patients $(71 \%)$ were culture-negative six months after completing a six month treatment regimen of oral fusidic acid and rifampin (4). Most other studies have focused on patients with incident MRSA infection rather than persistent infection. Macfarlane reported on $17 \mathrm{CF}$ patients with incident MRSA treated with oral and IV antibiotics. Utilizing a protocol which started with oral rifampicin and fusidic acid, they achieved a 94\% eradication rate at 12 months (5). A study by Solis of $15 \mathrm{CF}$ children treated with five days of oral and nebulized vancomycin was associated with a $55 \%$ eradication rate (6).

Key Questions to Be Answered about MRSA Treatment in CF. The key questions to be answered in future CF MRSA research can be divided into three main areas:

1) Most appropriate therapy for new MRSA infection and the impact of therapy on outcome.

a. Given that one third of new MRSA may spontaneously resolve does it need to be treated?

b. What is the most effective and safe regimen for eradication of new MRSA?

c. Does early aggressive treatment of new MRSA decrease the frequency of persistent MRSA infection and improve patient outcomes?

2) Most appropriate therapy for established MRSA infection and the impact of therapy on outcome.

a. Is eradication of persistent MRSA respiratory infection in CF possible?

b. What is the most effective treatment regimen for persistent MRSA respiratory infection in CF?

c. Does aggressive therapy of MRSA infection in CF reduce the frequency of exacerbations and aid in preserving lung function?

3) The effect of MRSA molecular characteristics and patient characteristics on treatment response and patient outcomes.

a. Are there MRSA molecular epidemiology characteristics which aid in predicting the persistence and severity of MRSA infection in CF?

b. Are there specific patient characteristics which aid in predicting the persistence and severity of MRSA infection in $\mathrm{CF}$ ?

c. What role does the environment play in determining the frequency and severity of MRSA infections in CF?

Clinical Trial Designs for MRSA in CF. There are currently studies in progress or about to be commenced which address each of the key areas described above. These include:
1) STAR-CF too Trial. (Staph $\underline{\text { Aureus }} \underline{\mathrm{R} e s i s t a n c e ~ i n ~} \underline{\mathrm{CF}}$,

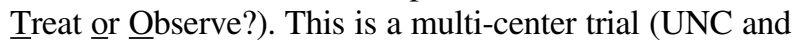
TDN coordinating center) with 6 months follow-up designed to assess if an early eradication protocol is effective for eradication of MRSA (and will provide opportunity to obtain data regarding spontaneous disappearance versus persistence of MRSA). Patients are assigned to either an observational arm or an interventional arm treated with two oral antibiotics, topical mupirocin, and environmental decontamination. Eighty patients with new respiratory tract MRSA infection will be enrolled and randomized 1:1. Primary outcome measure is proportion of subjects in each arm with MRSA negative respiratory cultures at day 28 . Secondary outcome measures include proportion of subjects treated with oral, inhaled and IV antibiotics over the 6 month study and number of days of use, and proportion of subjects with a protocol defined pulmonary exacerbation.

2) PMEP Trial. (Persistent Methicillin-resistant Staph Aureus Eradication Protocol) - This is a 6 month 2-center trial (Case and Johns Hopkins) in individuals $\geq$ age 12 designed to assess the effect of an aggressive 28-day inhaled and oral antibiotic combination protocol for CF individuals with persistent MRSA infection. Participants must have two positive MRSA respiratory cultures in the last 2 years at least 6 months apart and a positive MRSA respiratory culture at screening. Study design is a double-blind, comparatorcontrolled, parallel-group study with 1:1 assignment to either vancomycin for inhalation (250mg BID) or taste matched placebo. In addition, both groups will receive oral rifampin, a second oral antibiotic (TMPSMX or doxycycline), mupirocin intranasal cream and chlorhexidine body washes. Forty patients with persistent respiratory tract MRSA infection will be enrolled. Primary outcome measure is percentage of patients MRSA free by induced sputum respiratory tract culture one month after completion of 4-week eradication protocol in intervention arm vs control arm. Secondary outcome measures include proportion of patients MRSA free at 3 and 6 months after completion of treatment protocol, change in $\mathrm{FEV}_{1}$ from baseline on days 28 and 118, time to first exacerbation, and change in MRSA CFUs.

3) Characterization of MRSA infection/colonization in $C F$. While not a clinical trial, this is the largest ongoing study that seeks to define the molecular epidemiology characteristics of MRSA infections in CF and if molecular sub-types of MRSA differ in effect on exacerbations, lung function and nutrition. This is a multicenter study (coordinating center UNC) aiming to enroll 450 patients ages 1-18. Outcomes of interest: Characterize clinical impact of SCCmec MRSA sub-types on exacerbations and decline of $\mathrm{FEV}_{1}$ and nutrition using prospective and longitudinal study designs; Evaluate the best antibiotics to be used based on MRSA susceptibility obtained from different U.S. CF centers; Measure colo- 
nization rate in nose and skin in patients with known MRSA and in their main caregiver in a sub-group of CF patients with recent acquisition.

References: 1. CF Foundation Patient Registry. 2009 Annual Data Report. 2011. Bethesda, Maryland, CF Foundation.

2. Dasenbrook EC, Merlo CA, Diener-West M, et al. Persistent methicillin-resistant Staphylococcus aureus and rate of FEV1 decline in cystic fibrosis. Am J Respir Crit Care Med. 2008;178:814-21.

3. Dasenbrook EC, Checkley W, Merlo CA, et al. Association between respiratory tract methicillin-resistant Staphylococcus aureus and survival in cystic fibrosis. JAMA. 2010;303:2386-92.

4. Garske LA, Kidd TJ, Gan R, et al. Rifampicin and sodium fusidate reduces the frequency of methicillin- resistant Staphylococcus aureus isolation in adults with cystic fibrosis and chronic MRSA infection. J Hosp Infect. 2004;56:208-14.

5. Macfarlane M, Leavy A, McCaughan J, et al. Successful decolonization of methicillin-resistant Staphylococcus aureus in paediatric patients with CF using a three-step protocol. $\mathrm{J}$ Hosp Infect. 2007;65:231-6.

6. Solis A, Brown D, Hughes J, et al. Methicillinresistant Staphylococcus aureus in children with cystic fibrosis: An eradication protocol. Pediatr Pulmonol. 2003;36:189-95.

7. Doe SJ, McSorley A, Isalska B, et al. Patient segregation and aggressive antibiotic eradication therapy can control methicillin-resistant Staphylococcus aureus at large cystic fibrosis centres. J Cyst Fibros. 2010;9:104-9.

\title{
S8.1 \\ GENE MODIFIERS OF CF LUNG DISEASE: UPDATE ON MECHANISTIC INSIGHTS
}

\author{
Michael R. Knowles, M.D. \\ CF/Pulmonary Research \& Treatment Ctr., UNC at Chapel Hill, Chapel Hill, NC, USA
}

Cystic fibrosis $(\mathrm{CF})$ is a recessive "monogenic" disorder caused by mutations in CFTR. There is a wide range of lung disease severity in $\mathrm{CF}$, even for patients who are homozygous F508del. Studies of twins and sibs have determined that non-CFTR genetic factors account for $>50 \%$ of variability in lung disease (1). Identification of non-CFTR gene modifiers is particularly relevant in $\mathrm{CF}$, as almost all patients are now being diagnosed through neonatal screening programs. To intensively study gene modifiers, a North American CF Gene Modifier Consortium was formed by investigators at UNC/CWRU, Johns Hopkins, and Toronto, Canada. The Consortium developed a quantitative and standardized lung disease phenotype, based on multiple measures of $\mathrm{FEV}_{1}$ over 3 years, adjusted for survival (2). A GWAS (Illumina 610 Quad) was performed in 3,467 CF patients, and two loci with genome-wide significance were discovered (3). One locus was identified at chr 20q13.2 by linkage analysis of the Twin and Sibs (Hopkins) cohort (LOD score of 5.03), and SNPs under the linkage peak replicated in UNC/CWRU and Canadian association population patients. A robust association at chr $11 \mathrm{p} 13$ was discovered $\left(\mathrm{p}=3.3 \times 10^{-8}\right)$ in F508del homozygotes in UNC/CWRU and Canadian patients, and replicated in the family-based (Hopkins) population (joint analysis of three studies, $\mathrm{p}=1.5 \times 10^{-9}$ ). The significant SNPs at chr $11 \mathrm{p} 13$ are located in an intergenic region, 3' to $E H F$ (an epithelial-specific transcription factor) and APIP (an inhibitor of apoptosis) (4-7). $E H F$ is expressed in airway epithelia, and may serve as a regulator of differentiation under conditions of stress, and/or play a role in inflammation $(8,9)$. APIP inhibits apoptosis, and cis expression QTL (eQTL) patterns are reported for lymphocytes. The direction of the APIP eQTLs and "risk" SNPs in the GWAS suggest increased expression of APIP, and inhibition of apoptosis, is associated with worse CF lung disease. This is consistent with the concepts that delayed neutrophil clearance caused by reduced apoptosis can cause inflammation in the CF airways, and inhibition of apoptosis can contribute to mucus cell metaplasia $(10,11)$. The intergenic region at chr $11 \mathrm{p} 13$ has predicted regulatory features, including open chromatin domains. Ongoing work will test the hypothesis that genomic variation in this region alters expression of nearby genes, which has biologic relevance to CF airways disease. The study of genes that modify severity of "monogenetic" diseases, such as CF, is progressing rapidly, and mechanistic insights are beginning to emerge. The extension of the Consortium to trans-Atlantic (France) sites will provide additional power to identify new loci for studies to link genetic variation to mechanisms of lung disease severity.

References: 1. Vanscoy LL, Blackman SM, Collaco JM, et al. Heritability of lung disease severity in cystic fibrosis. Am J Respir Crit Care Med. 2007; 175:1036-43.

2. Taylor C, Commander CW, Collaco JM, et al. A novel lung disease phenotype adjusted for mortality attrition for cystic fibrosis genetic modifier studies. 
Pediatr Pulmonol. 2011 Apr 1. doi: 10.1002/ ppul.21456.

3. Wright FA, Strug LJ, Doshi VK, et al. Genomewide association and linkage identify modifier loci of lung disease severity in cystic fibrosis at $11 \mathrm{p} 13$ and 20q13.2. Nat Genet. 2011;43:539-46.

4. Cho DH, Hong YM, Lee HJ, et al. Induced inhibition of ischemic/hypoxic injury by APIP, a novel Apaf-1interacting protein. J Biol Chem. 2004;279:39942-50.

5. Cho DH, Lee HJ, Kim HJ, et al. Suppression of hypoxic cell death by APIP-induced sustained activation of AKT and ERK1/2. Oncogene. 2007;26:2809-14.

6. Oikawa T, Yamada T. Molecular biology of the Ets family of transcription factors. Gene. 2003;303:11-34.

7. Taniue K, Oda T, Hayashi T, et al. A member of the ETS family, EHF, and the ATPase RUVBL1 inhibit p53mediated apoptosis. EMBO Rep. 2011;12:682-9.
8. Silverman ES, Baron RM, Palmer LJ, et al. Constitutive and cytokine-induced expression of the ETS transcription factor ESE-3 in the lung. Am J Respir Cell Mol Biol. 2002;27:697-704.

9. Wu J, Duan R, Cao H, et al. Regulation of epithelium-specific Ets-like factors ESE-1 and ESE-3 in airway epithelial cells: potential roles in airway inflammation. Cell Res. 2008;18:649-63.

10. McKeon DJ, Condliffe AM, Cowburn AS, et al. Prolonged survival of neutrophils from patients with Delta F508 CFTR mutations. Thorax. 2008;63:660-1.

11. Harris JF, Fischer MJ, Hotchkiss JR, et al. Bcl-2 sustains increased mucous and epithelial cell numbers in metaplastic airway epithelium. Am J Respir Crit Care Med. 2005;171:764-72.

\title{
S8.2 \\ AN APICAL MEMBRANE HYPOTHESIS AND MECONIUM ILEUS SUSCEPTIBILITY IN CF
}

\author{
Johanna Rommens, Ph.D. ${ }^{1,2}$, Lei Sun ${ }^{3,5}$, Harriet Corvol, M.D., Ph.D., ${ }^{4,6}$, Pierre-Yves Boelle ${ }^{6,7}$, Pierre-François \\ Busson $^{7}$, Peter Durie ${ }^{8,9}$ and Lisa J. Strug, Ph.D. ${ }^{10,3}$ \\ 1. Program in Genetics \& Genome Biology, The Hospital for Sick Children, Toronto, ON, Canada; 2. Dept. of \\ Molecular Genetics, Univ. of Toronto, Toronto, ON, Canada; 3. Biostatistics Div., Dalla Lana School of Public \\ Health, Univ. of Toronto, Toronto, ON, Canada; 4. Dept. of Statistics, Univ. of Toronto, Toronto, ON, Canada; 5. \\ Dept. of Statistics, Univ. of Toronto, Toronto, ON, Canada; 6. Univ. Pierre et Marie Curie, Paris, France; 7. Dept. of \\ Biostatistics, AP-HP Hôpital St Antoine, Paris, France; 8. Program in Physiology \& Experimental Medicine, The \\ Hospital for Sick Children, Toronto, ON, Canada; 9. Dept. of Pediatrics, Univ. of Toronto, Toronto, ON, Canada; 10. \\ Program in Child Health Evaluative Science, The Hospital for Sick Children, Toronto, ON, Canada
}

Meconium ileus (MI) presents in $15 \%$ of CF patients, and is a highly heritable $(\mathrm{H}>0.88)$ and early complication. With the resources of the North American CF Gene Modifier Consortium (1), we sought to identify genes that contribute to MI susceptibility. Using the traditional genome-wide association study (GWAS) approach, two highly significant genome regions were identified with five SNPs with $\mathrm{p}$ values $<5 \times 10^{-8}$. The regions correspond to the SLC6A14 and SLC26A9 genes, and their SNP associations to MI have been replicated in additional North American and French CF patient sets. However, they explain only $5 \%$ of the MI phenotypic variability. To identify additional modifier genes that contribute to MI, we developed the hypothesis-driven GWAS (GWASHD) to reprioritize genotyping results considering current molecular understanding of CF. A major aspect of CF pathophysiology is impaired fluid and electrolyte flux at the epithelial interfaces of a number of CF-affected organs including the airway and intestine. The single cell epithelial layer is unique in that it forms a highly selective and tight barrier between body organ and surface or ductal interfaces. Epithelial function is achieved by cell polarization whereby many determinants and reg- ulators of fluid, solute and ion transport, including the CFTR ion channel reside specifically at the apical membrane. With these considerations, we proposed our "apical hypothesis" and used data from the Gene Ontology Consortium (2) to derive two lists of genes to test for relationship to MI susceptibility, one with gene products that localize to the apical membrane (155 genes), the second, with gene products that localize to the nuclear envelope (224 genes). The nuclear gene group showed no association to MI as anticipated, however, the "apical gene" group showed significant association, $\mathrm{p}$-value = 0.0002 . Together, the apical genes account for $17 \%$ of phenotypic variability in MI. Efforts to understand the biology of these contributing apical genes, and their relation to MI susceptibility and other CF phenotypes will be discussed.

References: 1. Wright FA, Strug LJ, Doshi VK, et al. Genome-wide association and linkage identify modifier loci of lung disease severity in cystic fibrosis at $11 \mathrm{p} 13$ and 20q13.2. Nat Genet. 2011;43:539-46.

2. Ashburner M, Ball CA, Blake JA, et al. Gene ontology: Tool for the unification of biology. The Gene Ontology Consortium. Nat Genet. 2000;25:25-9. 


\title{
S8.3
}

\section{GENETIC SUSCEPTIBILITY FOR CF-RELATED DIABETES IDENTIFIED BY GENOME-WIDE ASSOCIATION: THE INTERNATIONAL GENE MODIFIER CONSORTIUM}

\author{
Scott M. Blackman, M.D., Ph.D. \\ Pediatric Endocrinology, The Johns Hopkins Univ. School of Medicine, Baltimore, MD, USA
}

Diabetes develops frequently in people with cystic fibrosis over time, occurring in $25-50 \%$ of adolescents and adults with CF. People who fall into this subset of CF patients tend to have worse lung function, poorer nutritional status, and increased mortality. Treatment of diabetes (a.k.a. CF-related diabetes or CFRD) has been shown to correlate with improvement in lung function and body mass index.

CFRD develops as the capacity of the pancreatic beta cells to secrete insulin declines over time. In general, insulin resistance is not thought to play a substantial role, although CF patients can exhibit transient and significant drops in insulin sensitivity when ill or having a pulmonary exacerbation. At first, hyperglycemia may be transient, occurring only when ill or taking systemic glucocorticoid medications. Over time, insulin secretion diminishes, and daily exogenous insulin may become necessary. There is some evidence that insulin may benefit $\mathrm{CF}$ patients prior to meeting diagnostic criteria for CFRD.

The mechanisms underlying the development of diabetes in some CF patients are not well known. Diabetes occurs almost exclusively in CF patients with severe exocrine pancreatic insufficiency, suggesting that pancreatic autodigestion of islet cells may play a role. In support of this hypothesis, CF patients have demonstrated decreased glucagon and pancreatic polypeptide (made in the alpha and delta cells of the islets)(1). However, other lines of evidence suggest other factors are involved. First, the rate of CFRD varies widely even among CF patients with the same severe CFTR mutations (e.g., homozygous F508del). Second, epidemiologic data suggest that other factors (CF liver disease, transplant medications, ABPA) play a role, at least in a subset of people with CFRD(2). Third, studies of pancreatic tissue from diabetic and non-diabetic CF patients failed to detect correlation in CFRD status with islet cell mass or number. In general, pancreatic islets are preserved as the exocrine tissue becomes fibrotic. Interestingly, these studies found that a specific marker of CFRD was the presence of islet amyloid, a marker for beta cell dysfunction also specific to type 2 diabetes (T2D)(3). Finally, knock-out mouse studies have suggested there may be an intrinsic defect in CFTR-deficient pancreatic beta cells(4).

The identification of islet amyloid in CFRD islets suggested similarity to type 2 diabetes (T2D). T2D is caused at least in part by genes (40-60\% heritability) and bears some phenotypic and genetic similarity to CFRD. Patients who develop T2D have decreased sensitivity to insulin in the target tissues (e.g., skeletal muscle, liver, and fat cells). Initially, beta cell proliferation increases the insulin secretory capacity. Over time, however, beta cell function diminishes, resulting in hyperglycemia, and eventually, treatment with insulin. At diagnosis, patients with T2D may have lost $80 \%$ of insulin secretory capacity.

In the CF twin and sibling study, having a strong family history of T2D was correlated with a 3-fold increased risk of CFRD (5), suggesting that there may be genetic risk factors shared between T2D and CFRD. The degree to which variation in genes beyond CFTR (a.k.a. modifier genes, or genetic modifiers) affects CFRD, was tested in the CF Twin and Sibling study. Two phenotypes were considered: presence (vs. absence) of CFRD, and onset of CFRD by Cox regression (referred to as CFRDage). Comparison of concordance rates for CFRD and CFRDage between identical twins, non-identical twins and siblings with CF, showed that a high proportion (40-100\%) of the variation in CFRD risk was due to genetic modifiers(6).

Common variants in $\sim 40$ genetic loci have been associated with risk of T2D and with other glucose-related metabolic traits in people without CF. Initially using data from the CF Twin and Sibling study (TSS; Johns Hopkins University) and the Gene Modifiers Study (GMS; UNC Chapel Hill/Case Western), we found that a susceptibility gene for T2D, TCF7L2, also confers risk for CFRD(5). Those studies have since been replicated in a set of $\mathrm{CF}$ patients in the Canadian Consortium for $\mathrm{CF}$ genetic studies (CGS; University of Toronto), strengthening the overall evidence for this gene's involvement in CFRD (meta-analysis using the CFRDage trait, $n=4483$ patients, rs7903146 SNP, hazard ratio $=1.34$ per allele, $\mathrm{P}$ $=4.4 \times 10^{-7}$ ).

In addition, variants (single nucleotide polymorphisms or SNPs) in 6 additional risk loci for T2D were also tested for association with CFRD onset (CFRDage). These loci were selected based on effect size in T2D and estimated power for detection using $\sim 483 \mathrm{CF}$ patients. Study-wide significant association with CFRD was found for SNPs in CDKAL1 (rs7754840, $\mathrm{P}=1.7 \times 10^{-3}$; rs7756992, $\mathrm{P}=2.1 \times 10^{-5}$ ) and CDKN2A/B (rs1412829, $\mathrm{P}=1.2 \times 10^{-5}$ ). As was the case with the TCF7L2 SNP, the high-risk allele for T2D was the same as the high-risk allele for CFRD. The 4 remaining loci did not show statistically significant evidence for association with CFRD, (study-wide $\mathrm{P}<0.1$ in IGF2BP2; $\mathrm{P}>0.1$ in $\mathrm{KCNJ} 11$, HHEX, SLC30A8). Relatively weak associations with CFRD could have been missed due to limitations in study power. Susceptibility genes affecting both T2D and 
CFRD suggest that some pathways of disease pathogenesis are shared.

Using the families in the Twin and Sibling Study, we are also conducting a genome-wide linkage study for onset of CFRD. Linkage studies can be sensitive to both common and rare variants which affect the risk of disease. A genome-wide linkage map of 20,066 SNPs was typed for the TSS and met all quality-control measures. Nonparametric linkage (variance components) methods were used to detect linkage to the onset of CFRD using data from 122 phenotyped sibling pairs with $\mathrm{CF}$ and severe exocrine pancreatic insufficiency. The quantitative trait used for linkage was the Martingale residual for CFRD onset after Cox regression (CFRDresidual). This CFRD phenotype has identical heritability $(\sim 1.0)$ and correlation with pertinent covariates (e.g. sex, lung function, or T2D SNPs) as CFRDage. Empiric LOD scores were calculated by simulation. Genome-wide significant evidence for linkage was found at chromosome $2 \mathrm{q} 35$ 2q36.1 ( $\mathrm{LOD}=3.8$, approximately equivalent to studywide $\mathrm{P}=0.035)$. Genome-wide suggestive evidence for linkage was found at chromosome 12 p12 (LOD = 2.24). These studies identify a region of chromosome 2 which may harbor novel genetic modifiers of CFRD.

We are also conducting a genome-wide association study for CFRD onset using data from the International CF Gene Modifier Consortium. This type of study will be sensitive to common variants ( $>5 \%$ allele frequency in the population) of moderate effect size, similar to those already identified based on overlap with T2D susceptibility loci. We hypothesize that, in addition to loci shared with T2D which act on common diabetes pathways, there may be loci unique to CFRD which act on CFTR-specific or CFRD-specific pathways. Modifier loci in either category will present opportunities for future studies of diabetes pathogenesis and development of new therapies, both in CFRD and in T2D. Also, identification of a genetically at-risk subset of CF patients may have clinically relevant implications for CFRD screening strategies.
These studies show that the risk of CFRD is heavily influenced by genes other than CFTR. Three genetic modifiers of CFRD have been identified which are also susceptibility genes for type 2 diabetes, indicating that some pathways are shared between these two forms of diabetes. In general, these variants have associated with decreased insulin production and beta cell function in studies of non-CF patients, suggesting that beta cell function may be important in CFRD pathogenesis. Studies of CF patients, who are sensitized to develop diabetes at a young age, can inform on the pathophysiology of other forms of diabetes such as type 2 diabetes. Novel genetic modifiers of CFRD, such as may be found at chromosome 2q35-2q36.1, can identify molecular pathways of importance to either CFRD or T2D pathogenesis. Presented on behalf of the North American CF Gene Modifier Consortium. Supported by NIDDK, CFF, PES.

References: 1. Lanng S, Thorsteinsson B, Roder ME, et al. Pancreas and gut hormone responses to oral glucose and intravenous glucagon in cystic fibrosis patients with normal, impaired, and diabetic glucose tolerance. Acta Endocrinol (Copenh). 1993;128:207-14.

2. Marshall BC, Butler SM, Stoddard M, et al. Epidemiology of cystic fibrosis-related diabetes. J Pediatr. 2005;146:681-7.

3. Couce M, O'Brien TD, Moran A, et al. Diabetes mellitus in cystic fibrosis is characterized by islet amyloidosis. J Clin Endocrinol Metab. 1996;81:1267-72.

4. Stalvey MS, Muller C, Schatz DA, et al. Cystic fibrosis transmembrane conductance regulator deficiency exacerbates islet cell dysfunction after beta-cell injury. Diabetes. 2006;55:1939-45.

5. Blackman SM, Hsu S, Ritter SE, et al. A susceptibility gene for type 2 diabetes confers substantial risk for diabetes complicating cystic fibrosis. Diabetologia. 2009;52:1858-65.

6. Blackman SM, Hsu S, Vanscoy LL, et al. Genetic modifiers play a substantial role in diabetes complicating cystic fibrosis. J Clin Endocrinol Metab. 2009;94:1302-9.

\title{
S8.4 \\ MOUSE MODELS OF CF MODIFIERS
}

\author{
Mitchell L. Drumm, Ph.D. ${ }^{1}$, Lindsay Henderson ${ }^{2}$, Craig A. Hodges, Ph.D. ${ }^{1}$, Rebecca Darrah, Ph.D. ${ }^{1}$, Scott M. \\ Blackman, M.D., Ph.D. ${ }^{2}$, Peter Durie, M.D. ${ }^{4}$, Michael R. Knowles, M.D. ${ }^{3}$ and Garry R. Cutting, M.D. ${ }^{2}$ \\ 1. Depts. of Pediatrics \& Genetics, Case Western Reserve Univ., Cleveland, OH, USA; 2. Johns Hopkins Univ., \\ Baltimore, MD, USA; 3. UNC at Chapel Hill, Chapel Hill, NC, USA; 4. The Hospital for Sick Children, Toronto, \\ ON, Canada
}

Advances in genotyping technology have made it technically and financially feasible to carry out highdensity genotyping of polymorphisms simultaneously. Such advances provide the possibility to detect much more quickly than ever before genomic variants that influence, or cause, a phenotype. While these technolo- gies make it possible to identify disease-causing locations in the genome, narrowing the list of possible genes involved from approximately 25,000 to only a handful, the identity of the genes involved and how they impart their effects may not be immediately apparent. Functional assessment is critical and thus cellular and animal 
models are needed to confirm any suspected gene's effects.

For these types of studies, the mouse is ideally suited as it is possible to manipulate any suspected gene and place it in a CF context. In fact, there are hundreds of lines of mice with mutations in specific genes and hundreds of stem cells for the creation of additional lines, if desired. Thus, we have begun surveying putative $\mathrm{CF}$ modifying genes in mice by placing mutant forms of the modifiers on CF backgrounds.

One of the prominent features of all CF animal models (mouse, ferret and pig) is the propensity for intestinal obstruction in the distal ileum. Thus, modifiers of obstruction would be ideal for testing this concept. Blackman and colleagues used a family-based approach (linkage analysis) and found that several regions of the genome, including one on chromosome 8, significantly associate with meconium ileus in CF patients (1). Further analysis of the chromosome 8 region suggested that the gene involved is MSRA (Henderson, unpublished), encoding methionine sulfoxide reductase A, an enzyme that catalyzes the reduction of methionine sulfoxide to methionine. We introduced a null allele of MsrA into a CF line (Cftrtm1Unc) and compared the incidence of intestinal obstruction in CF mice with the three different MsrA genotypes. At 40 days, only $17 \%$ of CF mice $(n=30)$ with wildtype MsrA survived, while $42 \%$ of those herterozygous for the MsrA mutant allele $(n=33)$ and
$61 \%$ homozygous for the mutant allele $(n=46)$ survived. These results confirm the modifying effects of MSRA, but an enzyme that alters the state of methionine as a modifier opens a new avenue in our thinking about the pathophysiology of gastrointestinal obstruction in CF.

We have more recently reported a high density genotyping survey identifying two regions of the genome, one on chromosome 11 and one on 20, that associate significantly with CF lung disease (2). Neither of these regions neatly identifies a single gene and therefore we are in the process of examining several of the genes in those regions. To date, the most proximate genes to the chromosome 20 region are MC3R (melanocortin 3 receptor) and CBLN4 (cerebellin-like 4). We have obtained mice with mutant alleles of each of these genes and are currently crossing them with various $\mathrm{CF}$ lines to generate $\mathrm{CF}$ mice with different levels of modifier gene function. These mice are then put through a panel of assays to determine if altering putative modifier gene function has discernible effects on any CF-related phenotype.

References: 1. Blackman SM, Deering-Brose R, McWilliams R, et al. Relative contribution of genetic and non-genetic modifiers to intestinal obstruction in cystic fibrosis. Gastroenterology. 2006;131:1030-9.

2. Wright FA, Strug LJ, Doshi VK, et al. Genomewide association and linkage identify modifier loci of lung disease severity in cystic fibrosis at $11 \mathrm{p} 13$ and 20q13.2. Nat Genet. 2011;43:539-46.

\title{
S9.1 \\ QUALITY CONTROL OF NASCENT CFTR IN THE ENDOPLASMIC RETICULUM
}

\author{
Douglas M. Cyr, Ph.D. \\ Cell Biology, University of North Carolina, Chapel Hill, NC, USA
}

The fatal lung disease cystic fibrosis (CF) is a loss of protein function disorder caused by misfolding and premature degradation of the cystic fibrosis transmembrane conductance regulator (CFTR). CFTR is a $\mathrm{Cl}^{-}$channel that controls hydration of epithelial cell surfaces in airways and glands (1). Most CF patients inherit the CFTR $\Delta$ F508 mutant allele whose protein product exhibits subtle folding defects that lead almost all nascent forms to be degraded (2). Patients that exhibit partial CFTR function have mild CF symptoms, so restoration of CFTR $\Delta$ F508 activity to modest levels is a therapeutic goal.

F508 is located in NBD1 and is not essential for $\mathrm{Cl}^{-}$ conductance (1), but its deletion leads pools of nascent CFTR $\Delta$ F508 to accumulate in a foldable, but kinetically trapped conformation (3). CFTR $\Delta$ F508 misfolding appears to involve subtle defects in NBD1 folding that cause co-translational misassembly of an intermediate that is degraded rapidly and may not accumulate (4). Folding of the small pool of nascent CFTR $\Delta$ F508 that is spared initial degradation is arrested because of defective contact formation between NBD1 and regions that include intracellular loops exposed by MSD2 and misfolding of NBD2. Consequently 99\% of CFTR $\Delta$ F508 is degraded prematurely by the ubiquitin-proteasome system.

CFTR folding is assisted by several different molecular chaperones. The ER-associated Hsp40 Hdj-2 (DNAJA1) helps attract cytosolic Hsc70 to the ER membrane surface to facilitate co-translational folding and assembly of NBD1 (5). The ER luminal chaperone calnexin appears to act after $\mathrm{Hdj}-2$ to facilitate association of regions within CFTRs membrane-spanning and cytosolic domains (6). Terminal steps in folding of full-length CFTR are facilitated by Hsp90 and its associated co-factors (7).

Paradoxically, the selection of nascent forms of CFTR and CFTR $\Delta$ F508 for proteasomal degradation is also facilitated by molecular chaperones. The cytosolic E3 ubiquitin ligase CHIP interacts with Hsc70 and/or Hsp70 
to form a quality control machine that utilizes the polypeptide binding activity of Hsc/Hsp70 to target misfolded CFTR for proteasomal degradation (8). In addition, the ER-associated E3 RMA1/RNF5 acts in association with Derlin-1 and the E2 Ubc6e to ubiquitinate CFTR (4). How selection of CFTR for degradation by the RMA1 E3 machinery and CHIP E3 complex are synergized is not entirely clear. However, the RMA1 E3 complex may act coincident with translation to recognize folding defects in CFTR that involve the misfolding and defective assembly of NBD1 into a complex with the Rdomain $(4,6)$. In contrast, the CHIP E3 may act posttranslationally to recognize misfolded regions of CFTR that include NBD2 (4).

Deletion of F508 renders CFTR highly sensitive to changes in RMA1 activity, which suggests that the RMA1 E3 plays a critical role in selection of CFTR 4 F508 for premature degradation (4). Yet, it is unclear how the RMA1 complex distinguishes between on- and off-pathway forms of nascent CFTR. RMA1 interacts with the transmembrane quality control factor Derlin-1 and siRNA knockdown of Derlin-1 enhances cell surface expression of CFTR (4). Derlin-1 can form complexes with MSD1 of CFTR and Derlin-1 overexpression promotes ER retention and proteasomal degradation of CFTR (4). So, it is possible that Derlin-1 is part of a complex that acts as a membrane chaperone to scan the assembly status of CFTR's membrane regions and targets misassembled forms to RMA1 for ubiquitination. This seems logical, except the region in CFTR that is ubiquitinated by RMA1 is cytosolic. Therefore, quality control factors in addition to Derlin-1 may assist RMA1 in the selection of misfolded CFTR for ubiquitination. Clues to the identity of such a factor come from the analysis of the Hsp40/DnaJ family of Hsc70 co-chaperones. Hsp40 proteins utilize a conserved J-domain to regulate Hsc70 ATPase activity and specify client proteins of Hsc70. One mechanism for specification of Hsc70 function is for a specialized Hsp40 to attract Hsc70 to function in a discrete cellular location. Studies in yeast identify a unique Hsp40 sub-type that is integrated into the ER membrane and exposes its J-domain to the cytosol (9). This sub-family is represented in yeast by HLJ1 (high copy lethal DnaJ 1) and deletion analysis suggests that HLJ1 functions with cytosolic Hsc70 in ER quality control (10). RMA1 is not expressed in yeast, so we pondered whether or not an HLJ1-like protein might act with RMA1 in human cells to assist in the selection of CFTR for degradation. Indeed, HLJ1-like Hsp40s are conserved in the human genome and this talk will describe the characterization of human DNAJB12 (JB12). JB12 is a Type II Hsp40 that contains a J-domain and $\mathrm{G} / \mathrm{F}$-like region and is similar to HLJ1 in that it is localized to the ER membrane and exposes its J-domain to the cytosol. Yet, JB12 differs from HLJ1 in that it contains $\mathrm{N}$ - and C-terminal extensions that were added during the course of evolution. Data presented will demonstrate that JB12 acts with Hsc70 and RMA1 to facilitate proteasomal degradation of CFTR and CFTR $\Delta$ F508. JB12 forms complexes that contain RMA1 and Derlin-1 and increasing JB12 levels dramatically increases association of Hsc70 with ER localized forms of CFTR and RMA1. Depletion of endogenous JB12 results in a significant 3-fold increase in the folding efficiency of CFTR and permits a pool of CFTR $\Delta F 508$ to escape the ER. JB12 appears to direct Hsc70 to function with the E3 RMA1 in degradation of nascent CFTR $\Delta$ F508 and CFTR. Since fluctuations in its activity impact on the fate of nascent CFTR, JB12 is capable of exerting control over the folding efficiency of nascent CFTR and CFTR $\triangle F 508$ and JB12. Inactivation of JB12 increases the potency of small molecule folding correctors and JB12 appears to be a therapeutic target for treatment of $\mathrm{CF}$.

References: 1. Rowe SM, Miller S, Sorscher EJ. Cystic fibrosis. N Engl J Med. 2005;352:1992-2001.

2. Cyr DM. Arrest of CFTRDeltaF508 folding. Nat Struct Mol Biol. 2005;12:2-3.

3. Younger JM, Ren HY, Chen L, et al. A foldable CFTR \{Delta\}F508 biogenic intermediate accumulates upon inhibition of the Hsc70-CHIP E3 ubiquitin ligase. J Cell Biol. 2004;167:1075-85.

4. Younger JM, Chen L, Ren HY, et al. Sequential quality-control checkpoints triage misfolded cystic fibrosis transmembrane conductance regulator. Cell. 2006;126:571-82.

5. Meacham GC, Lu Z, King S, et al. The Hdj2/Hsc70 chaperone pair facilitates early steps in CFTR biogenesis. EMBO J. 1999;18:1492-505.

6. Rosser MF, Grove DE, Chen L, et al. Assembly and misassembly of cystic fibrosis transmembrane conductance regulator: folding defects caused by deletion of F508 occur before and after the calnexin-dependent association of membrane spanning domain (MSD) 1 and MSD2. Mol Biol Cell. 2008;19:4570-9.

7. Loo MA, Jensen TJ, Cui L, et al. Perturbation of Hsp90 interaction with nascent CFTR prevents its maturation and accelerates its degradation by the proteasome. EMBO J. 1998;17:6879-87.

8. Meacham GC, Patterson C, Zhang W, et al. The Hsc70 co-chaperone CHIP targets immature CFTR for proteasomal degradation. Nat Cell Biol. 2001;3:100-5.

9. Walsh P, Bursac D, Law YC, et al. The J-protein family: modulating protein assembly, disassembly and translocation. EMBO Rep. 2004;5:567-71.

10. Youker RT, Walsh P, Beilharz T, et al. Distinct roles for the Hsp40 and Hsp90 molecular chaperones during cystic fibrosis transmembrane conductance regulator degradation in yeast. Mol Biol Cell. 2004;15:4787-97. 


\title{
S9.2 \\ BRUSH BORDER MYOSINS AND CFTR IN THE INTESTINE: MOTORS AND TETHERS?
}

\author{
Nadia A. Ameen, MBBS \\ Pediatrics, Yale University School of Medicine, New Haven, CT, USA
}

Cystic fibrosis transmembrane conductance regulator (CFTR) chloride channels are in the apical domain of both crypt and villus enterocytes where they play a critical role in anion and fluid secretion. Genetic mutations that lead to absence of functional CFTR on the apical plasma membrane of enterocytes result in intestinal obstruction and cystic fibrosis. On the other hand, enterotoxins elaborated by Vibrio cholerae and Escherichia coli utilize CFTR as their apical exit pathway to elicit massive fluid secretion and secretory diarrhea following cAMP and cGMP signaling $(1,2)$. In native epithelium and cultured polarized intestinal cells, both the number of CFTR channels and anion transport on the cell surface are regulated by protein kinase A, protein kinase Gphosphorylation and endocytic recycling and exocytosis of CFTR-containing vesicles by membrane trafficking $(3,4)$ (Fig. 1). But the mechanisms that control the intracellular trafficking itinerary of CFTR in intestinal cells are poorly understood. Polarized intestinal epithelial cells possess an apical brush border (BB) with tightly packed microvilli or membrane protrusions on their apical surface. Microvilli increase the apical surface area of enterocytes and are supported by bundled arrays of core actin filaments $(\sim 20)$ and cross-linking proteins that provide the structural support for each microvillus. The microvillus core rootlets descend into the apical cytoplasm of the enterocyte at the terminal web (TW), a region rich in cross-linking filaments composed of myosin and spectrin (5) (Fig.2). Members of the unconventional myosin family of actin binding proteins are abundant in intestinal epithelial cells where they play major roles as cargo transporters and as mediators of membrane-cytoskeleton adhesion. The subcellular localization, known functions and diseases associated with intestinal myosins are listed in Table 1. The distribution of CFTR along the microvillar membranes, in clathrincoated pits and vesicles, apical recycling and exocytic vesicles in the native enterocytes, suggest a role for myosins in regulating intracellular traffic (6). Myosin 6 is the only myosin family member that has been shown to associate with CFTR in the intestine. This minus end directed motor is unique among actin-based motors because it moves away from the plasma membrane. In cultured cells, myosin 6 associates with clathrin coated pits and coated vesicles and interacts with clathrin, the endocytic adaptors AP-2 and the C terminus of disabled 2 (Dab2) to regulate clathrin-mediated endocytosis(7). In the intestine, myosin 6 is present in both crypt and villus enterocytes and localizes to the lower third of microvilli, the terminal web and intermicrovillar domain, the sites of clathrin-coated pit formation and initiation of apical endocytosis. Absence of myosin 6 leads to decreased negative membrane tension in the enterocyte $\mathrm{BB}$ microvillus and intermicrovillar membrane in myosin 6 mutant mice (Myo6 sv/sv) (Fig. 2, 3), markedly delayed apical endocytosis of CFTR, redistribution of the endocytic adaptor Dab2 in the terminal web region, and exaggerated fluid secretion following treatment with $E$. coli enterotoxin due to accumulation of CFTR on the surface of crypt and villus enterocytes in the small intestine $(8,9)$. Myosin 6 was suggested as the candidate motor that would move CFTR down the microvillar membrane and sequester channels into clathrin coated pits for endocytosis, but its distribution in the enterocyte brush border does not support such a role. How CFTR migrates down the microvillus to the intermicrovillar membrane of enterocytes in preparation for endocytosis remains unknown. Myosin 6 distribution in the enterocyte BB contrasts to rat kidney proximal tubules, where it localizes along the entire length of microvilli, and plays a role in redistribution of NHE3 $\mathrm{Na}^{+} / \mathrm{H}^{+}$exchanger along the microvilli during acute hypertension(10).

Myosin 1a (Myo1a) is the major plus end actin motor and counterpart to myosin 6 in the enterocyte BB. In contrast to myosin 6 that is present in both crypt and villus enterocytes, Myola is essentially confined to the $\mathrm{BB}$ of mature villus enterocytes in small intestine where it bridges the actin core to the microvillar membrane (Fig.3). Myosin 1a is in lipid rafts, binds to and plays a critical role in tethering and retention of the $\mathrm{BB}$ hydrolase sucrase isomaltase (SI) in the microvillar membrane(11). Lack of this plus end motor in Myola KO mice leads to bnormalities in microvillar membrane tension, blebbing and abnormal membrane protrusion in BB of villus enterocytes in the small intestine (Fig.3). Preliminary studies of CFTR localization and anion transport in Myola $\mathrm{KO}$ mice indicate that CFTR accumulates in the terminal web region, is absent from the $\mathrm{BB}$ and redistributes to the basolateral domains of villus enterocytes in the small intestine. Analysis of CFTR anion transport (Ussing chambers) in intestinal tissues from Myola $\mathrm{KO}$ mice reveal a marked $(>50 \%)$ reduction in CFTR current (Isc) compared to WT controls but only in intestinal segments that possess villi. Ongoing studies will seek to determine the specific role that Myola plays in regulating CFTR localization and function in the enterocyte BB. 
References: 1. Field M, Semrad CE. Annu Rev Physiol. 1993;55:631-55.

2. Ameen N, Alexis J, Salas P. Histochem Cell Biol. 2000;114:69-75.

3. Golin-Bisello F, Bradbury N, Ameen N. Am J Physiol Cell Physiol. 2005;289:C708-16.

4. Silvis MR, Bertrand CA, Ameen N, et al. Mol Biol Cell. 2009;20:2337-50.

5. Mooseker MS. Annu Rev Cell Biol. 1985;1:209-41.

6. Ameen NA, van Donselaar E, Posthuma G, et al. Histochem Cell Biol. 2000;114:219-28.

7. Buss F, Kendrick-Jones J. Biochem Biophys Res Commun. 2008;369:165-75.

8. Ameen N, Apodaca G. Traffic. 2007;8:998-1006.

9. Collaco A, Jakab R, Hegan P, et al. J Biol Chem. 2010;285:17177-87.

10. Yang LE, Maunsbach AB, Leong PK, et al. J Am Soc Nephrol. 2005;16:2890-6.

11. Tyska MJ, Nambiar R. Commun Integr Biol. 2010;3:64-6.

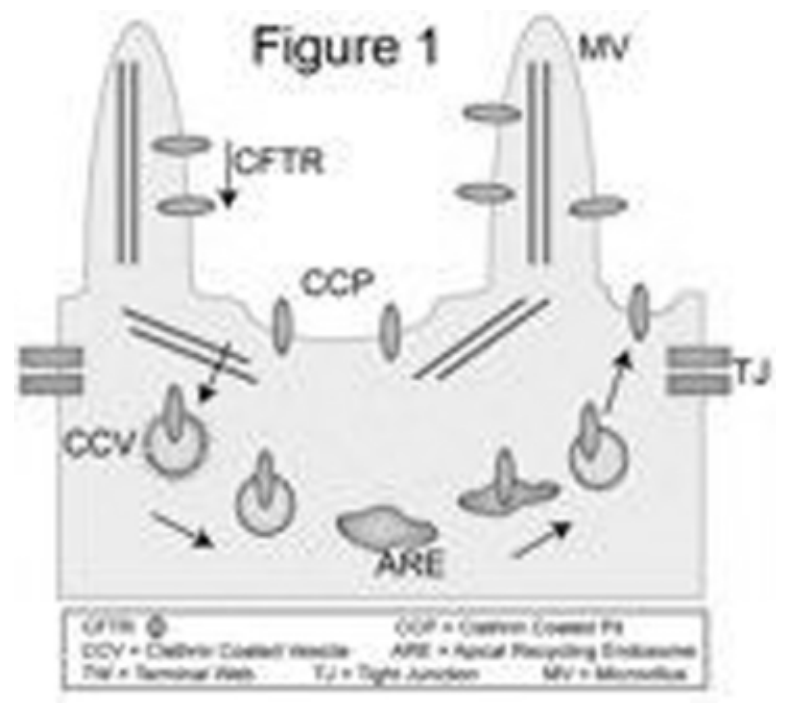

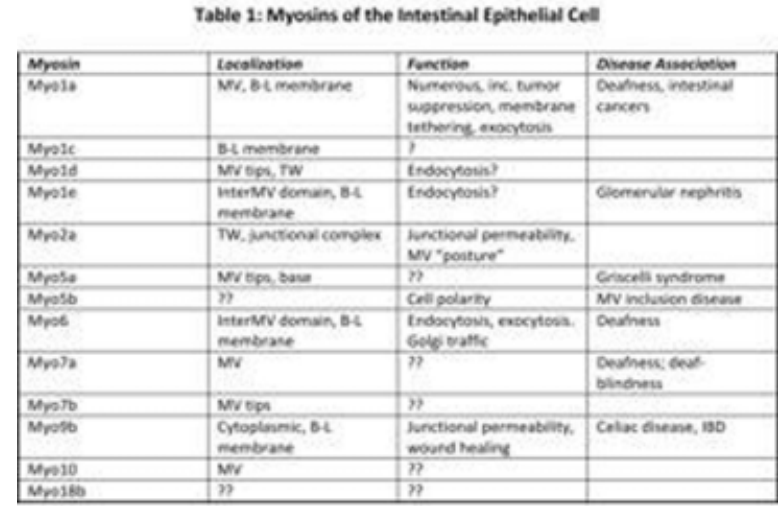
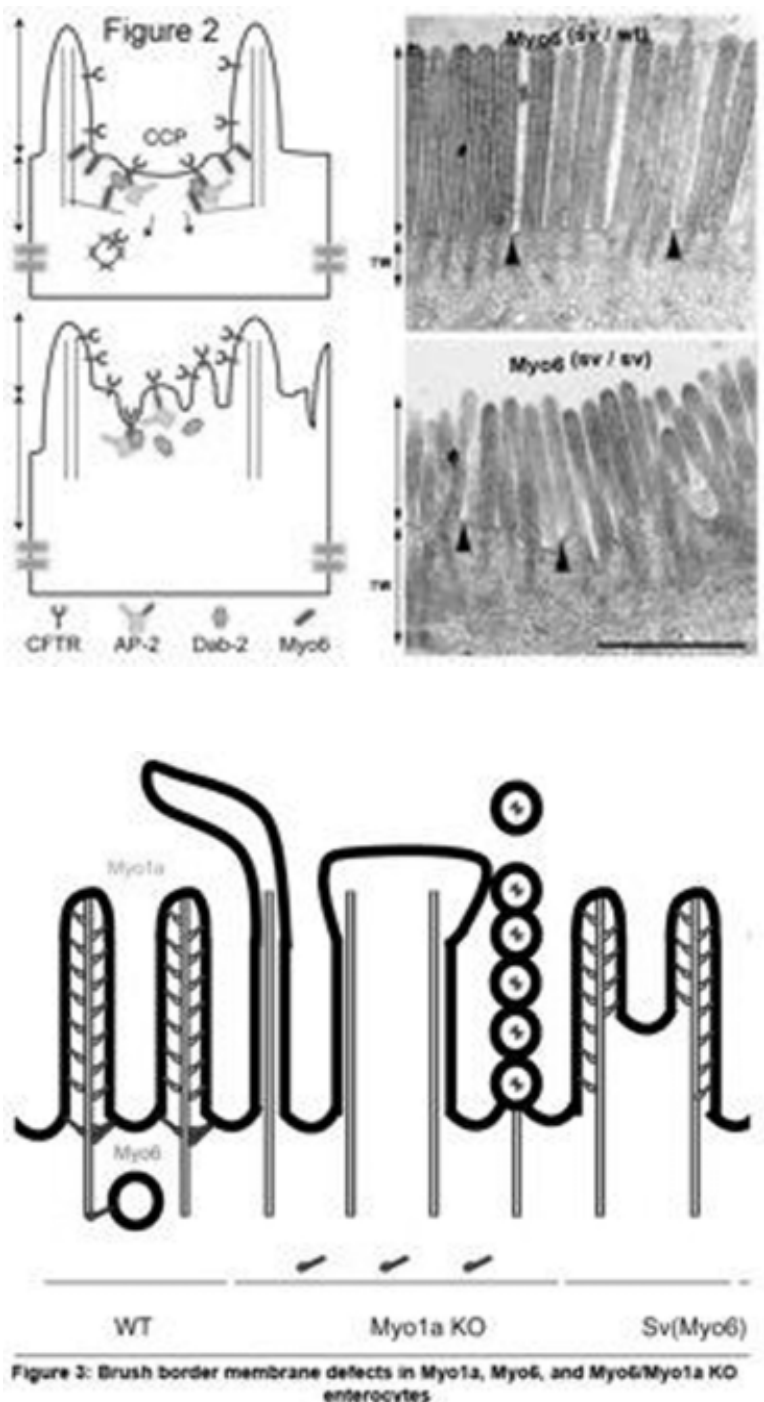


\title{
S9.3 ROLE OF ADAPTOR PROTEINS IN CFTR DOWN-REGULATION
}

\author{
Lianwu Fu, Ph.D., Andras Rab, Zsuzsa Bebok, M.D. and James F. Collawn, Ph.D. \\ Dept. of Cell Biology, Univ. of Alabama, Birmingham, AL, USA
}

Cell surface proteins often contain short (4-6 amino acid) cytoplasmic tail signals that allow for their efficient entry into clathrin-coated pits and clearance from the cell surface (reviewed in (1)). The two most common types of internalization signals consist of a tyrosine-based signal (NPXY or YXX $\Phi$ ) and a di-leucine-based signal (D/EXXXLL/I) where $X$ is any amino acid and $\Phi$ is a large hydrophobic residue. For the cystic fibrosis transmembrane conductance regulator (CFTR), two such signals have been identified in the C-terminal tail, YDSI and DSIQKLL $(2,3)$. There is evidence that the YDSI and DSIQKLL signals are recognized by the adaptor protein2 (AP-2) complex at the cell surface (3-5). Interestingly, both tyrosine-based and di-leucine-based signals have been shown to act as lysosomal targeting signals (6). Furthermore, earlier studies indicate that ubiquitination can serve as an internalization signal as well as a signal for lysosomal targeting $(6,7)$, making it difficult to differentiate between internalization and lysosomal targeting signals.

The AP-2 complex is composed of alpha-2, beta-2, mu-2 and sigma- 2 subunits. AP-2 provides two functions: (1) it recognizes and binds to the internalization signal; and (2) it binds to and promotes clathrin assembly. The beta- 2 subunit binds to clathrin, the mu- 2 binds to the internalization signal of the cell surface protein, and the alpha- 2 subunit recruits accessory proteins that are important for coated-pit formation and scission (reviewed in (6)). For CFTR, there is evidence that the tyrosine-based signal interacts with AP-2. Weixel and Bradbury demonstrated that a photoactivatable peptide containing the YDSI sequence could be UV cross-linked to the $50 \mathrm{kDa}$ mu-2 subunit of the AP-2 complex. Furthermore, they demonstrated that overexpression of a dominant negative mu-2 subunit inhibited CFTR internalization in HeLa cells (4). More recent studies in HEK cells by Collaco and Ameen demonstrated that the alpha2 subunit was important for CFTR internalization and importantly, GST pull-down assays indicated that the alpha-2 subunit is a binding partner for CFTR (5). Although it is unclear if both subunits of the AP-2 complex interact directly with the cytoplasmic tail of CFTR, both studies support the idea that the AP-2 directly interacts with CFTR and this interaction is important for the clearance of CFTR from the cell surface.

In our studies, we investigated the relationship between CFTR endocytosis and CFTR protein half-life. In that regard, we examined two different types of mutations in CFTR, one in which we ablated the tyrosinebased signal in CFTR, and another which included 2 nat- urally occurring CFTR mutations, R31L and R31C. In the first, we mutated the YDSI sequence to ADSA and found that CFTR internalization was reduced by more than $75 \%$, yet this change had no effect on CFTR protein half-life (8). In the second, we noticed that both R31 mutants appeared to create potential internalization signals, e.g., YRQR31 -> YRQC31 or YRQL31. Analysis of these CFTR mutants in endocytosis assays revealed that both mutants were internalized at double the rate of the wild type protein. More importantly, the mutations had no effect on the protein half-life (9). And finally, studies by Bradbury demonstrated that another naturally occurring mutation, N287Y, generated a tyrosine-based signal in CFTR's cytoplasmic loop 2. The appearance of this second internalization signal almost doubled the rate of CFTR internalization and again, this mutation had no effect on CFTR half-life (10). These studies indicated that CFTR mutations that introduced a tyrosine-based signal dramatically affected internalization, but had no effect on CFTR half-life, indicating that the two processes are not necessarily coupled.

Based on the above studies, it appeared that AP-2 interactions were not critical for CFTR down-regulation. Furthermore, it is clear that CFTR endocytosis is affected by a number of proteins besides AP-2. Details regarding the role of these accessory proteins in CFTR endocytosis are now beginning to be appreciated. Swiatecka-Urban, Stanton and colleagues demonstrated that when CFTR was immunoprecipitated from polarized Calu-3 cells, a number of proteins were brought down in a complex that includes myosin VI, Dab2, and clathrin (11). They also showed that myosin VI, a minus-end directed actin motor, was important for CFTR internalization by demonstrating that a dominant-negative form of myosin VI interfered with CFTR internalization in HEK293 cells. Further support for a myosin VI role in endocytosis came from studies by Ameen and Apodaca in intestinal cells from myosin VI-deficient mice (12). Coimmunoprecipitation studies performed in polarized intestinal $\mathrm{CaCo}-2$ cells illustrated that endogenous CFTR brought down a complex that included Dab2, alpha-AP-2, myosin VI, and clathrin (5). Furthermore, Collaco and Ameen demonstrated that in the intestine, CFTR interacts indirectly with Dab2 through the AP-2 complex (alpha-2 subunit) (5).

Dab2 (Disabled 2) is particularly interesting because like AP-2 it can act as an adaptor protein. Maurer and Cooper demonstrated that Dab2 functions as an adaptor independently from AP-2 in LDL receptor internalization (13). Furthermore, they showed that Dab2 is important in megalin internalization in visceral endoderm cells 
(14). Both of these receptors have an NPXY type tyrosine-based signal. Interestingly, apical CFTR is increased in the intestines of Dab2 knockout mice, suggesting that it is important in CFTR internalization and/or down-regulation (5). In this talk, we will present a model to explain the roles of AP-2 and Dab2 in CFTR endocytosis and lysosomal targeting in polarized human airway epithelial cells. We will also discuss how these two adaptors differ with regard to their effects on CFTR down-regulation.

References: 1. Trowbridge IS, Collawn JF, Hopkins CR. Signal-dependent membrane protein trafficking in the endocytic pathway. Annu Rev Cell Biol. 1993;9:129-61.

2. Prince LS, Peter K, Hatton SR, et al. Efficient endocytosis of the CFTR requires a tyrosine-based signal. J Biol Chem. 1999;274:3602-9.

3. Hu W, Howard M, Lukacs GL. Multiple endocytic signals in the C-terminal tail of the CFTR. Biochem J. 2001;35:561-72.

4. Weixel KM, Bradbury NA. Mu 2 binding directs the CFTR to the clathrin-mediated endocytic pathway. J Biol Chem. 2001;276:46251-9.

5. Collaco A, Jakab R, Hegan P, et al. Alpha-AP-2 directs myosin VI-dependent endocytosis of CFTR chloride channels in the intestine. J Biol Chem. 2010;285:17177-87.

6. Bonifacino JS, Traub LM. Signals for sorting of transmembrane proteins to endosomes and lysosomes. Annu Rev Biochem. 2003;72:395-447.
7. Sharma M, Pampinella F, Nemes C, et al. Misfolding diverts CFTR from recycling to degradation: quality control at early endosomes. J Cell Biol. 2004;164:923-33.

8. Peter K, Varga K, Bebok Z, et al. Ablation of internalization signals in the carboxyl-terminal tail of the CFTR enhances cell surface expression. J Biol Chem. 2002;277:49952-7.

9. Jurkuvenaite A, Varga K, Nowotarski K, et al. Mutations in the amino terminus of the CFTR enhance endocytosis. J Biol Chem. 2006;281:3329-34.

10. Silvis MR, Picciano JA, Bertrand C, et al. A mutation in the CFTR generates a novel internalization sequence and enhances endocytic rates. J Biol Chem. 2003;278:11554-60.

11. Swiatecka-Urban A, Boyd C, Coutermarsh B, et al. Myosin VI regulates endocytosis of the CFTR. J Biol Chem. 2004;279:38025-31.

12. Ameen N, Apodaca G. Defective CFTR apical endocytosis and enterocyte brush border in myosin VIdeficient mice. Traffic. 2007;8:998-1006.

13. Maurer ME, Cooper JA. The adaptor protein Dab2 sorts LDL receptors into coated pits independently of AP-2 and ARH. J Cell Sci. 2006;119:4235-46.

14. Maurer ME, Cooper JA. Endocytosis of megalin by visceral endoderm cells requires the Dab2 adaptor protein. J Cell Sci. 2005;118:5345-55.

\title{
S9.4 \\ REPROGRAMMING PROTEOSTASIS AND CFTR FOLDING TO MANAGE CYSTIC FIBROSIS
}

\author{
William E. Balch, Ph.D. \\ Cell Biology/Chemical Physiology, The Scripps Research Institute, La Jolla, CA, USA
}

The cell exploits the environment sensitive (1) and emergent properties of proteostasis (2-4) to generate and maintain proteome balance (3) in diverse cell, tissue and organismal systems during development and in response to aging and disease such as cystic fibrosis (CF). The proteostasis network (PN) is a system of signaling pathways (UPR*, HSR*, ARE* and IR*), and chaperone (Hsp40/70/90, chaperonins, and sHSPs*) and degradative components (UPS*, ALES*) that respond to environmental stress through genetic and epigenetic mechanisms to manage folding and membrane trafficking (5). Physical, pathological and inherited challenges to the energetics of the biological fold (its "landscape") can compromise proteome balance (2). Our goal is to understand how the inherited misfolding prone protein $\triangle F 508$ CFTR is managed by proteostasis.

By use of systems level proteomic, genomic and chemical tools we are building a dynamic, multi-layered view of the healthy CFTR biological protein fold and the changes that occur in response to energetically compromised folding stress such as is observed in CF and $\triangle \mathrm{F} 508$. We provide evidence that chemical biology man- agement of the PN can alter the composition of the local proteostasis program to restore function. The discovery of tools that redirect the $\triangle \mathrm{F} 508$ fold to function highlights the potential value of the emergent properties of the proteostasis network to therapeutically rebalance the epithelial cell proteome to potentially benefit CF patient health span.

References: 1. Powers ET, Balch WE. Protein folding: Protection from the outside Nature. 2011;471:42-3.

2. Balch WE, Morimoto RI, Dillin A, et al. Adapting proteostasis for disease intervention. Science. 2008;319:916-9.

3. Hutt D, Balch WE. Cell Biology. The proteome in balance. Science. 2010;329:766-7.

4. Balch WE, Roth DM, Hutt DM. Emergent properties of proteostasis in managing cystic fibrosis. Cold Spring Harb Perspect Biol. 2011;3(2):pii: a004499.

5. Hutt DM, Herman D, Rodrigues AP, et al. Reduced histone deacetylase 7 activity restores function to misfolded CFTR in cystic fibrosis. Nat Chem. Biol. 2010;6:25-33. 
*UPR (unfolded protein response); HSR (heat shock response); ARE (anti-oxidant response elements); IR (inflammatory response); UPS (ubiquitin-proteasome system); sHSPs (small heat shock proteins); ALES (autophagy-lysosomal-endosomal system).

\title{
S10.1 \\ ANTIBIOTIC SELECTION AND DURATION OF THERAPY FOR PULMONARY EXACERBATIONS IN CF
}

\author{
J. Stuart Elborn, M.D., FRCP \\ Centre for Infection \& Immunity \& Adult CF Ctr., Queen's Univ. of Belfast, Belfast, United Kingdom
}

In people with cystic fibrosis pulmonary exacerbations are associated with a significant reduction in quality of life, more rapid decline in $\mathrm{FEV}_{1}$ and reduced survival (1). There is a good evidence base to help with treatment decisions for chronic treatment, mostly aimed at improving $\mathrm{FEV}_{1}$ and reducing pulmonary exacerbations (2). However, the evidence base for many aspects of the treatment of pulmonary exacerbations is less extensive. The important issues of 1) when to treat; 2) which antibiotics and other therapies to use; 3) how long to treat; and 4) what to do at the end of a pulmonary exacerbation, are largely based on custom and practice. Most published studies on exacerbations are observational with few studies exploring and comparing alternate options.

When to start treatment? Defining pulmonary exacerbations has been a subject of much effort in the past 10 years (3). A number of constructs have been developed to help with a definition that will be useful particularly in clinical trials but there has been less focus on a pragmatic definition for use in clinical decision making. A tool developed in the Akron CF Centre has shown some utility in focusing physicians on the symptoms of pulmonary exacerbation and in one study has resulted in more consistent initiation of antibiotic therapy (4).

Significant variation in decision making to initiate antibiotics was seen in a recent vignette-based study (5). Systemic symptoms and measures of pulmonary function were more strongly related to a decision to treat with IV antibiotics while symptoms of cough and sputum and crackles on exacerbation were less important. It may also be that people with $\mathrm{CF}$ perceive an exacerbation differently to clinicians.

Defining an exacerbation for clinical practice should focus on operationally describing the symptoms that in an individual should be considered an indication to start IV antibiotics. These may be heterogeneous and need to be individualised for each patient. It is likely that a low threshold for initiation of antibiotic therapy is best practice, as better performing centres use more antibiotics (5).

Oral, inhaled or intravenous? The decision to commence antibiotics for an exacerbation is determined by severity of the symptoms and clinical signs and physio- logical measurements associated with the exacerbation and by the organism(s) identified. Most exacerbations in people with CF not chronically infected with Pseudomonas aeruginosa are associated with Staphylococcus aureus or Haemophilus influenzae, though on some occasions no bacteria are isolated by conventional methods (6). These organisms can be effectively treated with an appropriate dose of oral antibiotics directed against these organisms. This is usually higher than in non-CF situations. Treatment should be started empirically and then adjusted accordingly when the organism has been identified and anti-microbial susceptibility testing undertaken. Methicillin resistant Staphylococcus aureus (MRSA) associated exacerbations can be treated with oral antibiotics but when severe may require intravenous treatment.

For patients with $P$. aeruginosa infection and a mild exacerbation, when there is no change in lung function and only a modest increase in cough and sputum, treatment with an oral agent such as ciprofloxacin is a rational approach. For exacerbations with severe symptoms or a significant reduction in $\mathrm{FEV}_{1}$, intravenous antibiotics are indicated. There are no data to support the use of inhaled antibiotics for pulmonary exacerbations, though a number of centres use inhaled tobramycin instead of an intravenous aminoglycoside in patients who have impaired renal function. Treatment of exacerbations associated with other Gram negative infections or nontuberculous mycobacterial infection is challenging and may require the use of oral, intravenous and inhaled therapies (7).

Choice of intravenous antibiotic. Most CF guidelines recommend combining an antipseudomonal extended-action penicillin, cephalosporin, monobactam or carbapenem and aminoglycoside for the treatment of a pulmonary exacerbation (6). The rationale for this combination is largely to prevent the development of resistance. Again, doses are usually higher than those normally used (6). There is no good evidence that single agent therapy is better or worse than dual agent therapy but the resistance concerns remain with single agent therapy. The role of anti-microbial sensitivity testing to direct antibiotic choice is an area of some controversy. The accuracy of sensitivity testing has been questioned and in 
one study there was no impact on effectiveness of treatment whether antibiotics were used to which the $P$. aeruginosa was sensitive compared to resistant (8). Synergy testing has been explored in multi-resistant $P$. aeruginosa and Burkholderia cepacia complex organisms but again appears to offer no advantage over an empirical decision (9). However, recent work from our CF centre has suggested that failure to recover from a pulmonary exacerbation is more likely if the treatment is with antibiotics to which the patient's $P$. aeruginosa is not sensitive. Pragmatically, antibiotic sensitivity testing may be considered a guide to antibiotic choice but other considerations such as allergy to antibiotics, previous good response and patient preference should also be considered.

Length of time for IV antibiotics. A few studies have addressed this particular issue. Most of the improvement in $\mathrm{FEV}_{1}$ and other metabolic and inflammatory markers improves after 7 days of IV antibiotics (10). This was confirmed in a large study of $\mathrm{FEV}_{1}$ recovery which demonstrated almost all of the recovery was achieved within 14 days (11). This is difficult to study as convention has been to treat for 14 days. However, this study suggests that it is reasonable in practice for IV antibiotic courses to be for 10-14 days depending on response. If there is full resolution of symptoms, inflammatory markers (white cell count and CRP) and full recovery of $\mathrm{FEV}_{1}$, then a 10-day course is a reasonable choice. For exacerbations which are not resolving there is very little data to help from studies for decision making. At some centres the IV course is continued for 3 or 4 weeks while an alternative approach is to change the IV combination for a further 2 weeks and monitor progress. Management of exacerbations should be complemented by increased airway clearance, consideration of nutritional loss, active management of blood glucose, and correction of hypoxia and hypercapnia, if required. There is no evidence to support the routine additional use of oral corticosteroids.

What to do at the end of a pulmonary exacerbation? All patients should have their chronic therapy reviewed at the end of an exacerbation. Consideration of factors that may have contributed to the episode should include adherence to regular therapies, undertreatment and new complications such as diabetes. The importance of prevention of exacerbations should be discussed and strategies implemented to reduce further episodes and if they do develop, early initiation of therapy.

Microbial diversity and exacerbations. A number of recent studies have demonstrated that there is a large number of different bacterial genera in the sputum from people with CF (12). These include a range of anaerobic bacteria, Streptococci species, as well as viruses and fungi. The relevance of these observations to clinical care is currently unclear. Molecular information from nonculture based methods of identification may become increasingly available and may prove to be a useful tool in determining the most appropriate antibiotic therapy for exacerbations. Several current ongoing studies may help to clarify this.

Conclusions. Treatment of pulmonary exacerbations remains a major part of cystic fibrosis care, yet is an area in which we do not have sufficient studies to allow us to optimise care. It is clear that exacerbations are associated with poorer outcomes and studies focused on how to maximise the treatment of exacerbations will be important.

References: 1. de Boer K, Vandemheen KL, Tullis E, et al. Thorax. 2011;66:680-5.

2. Flume PA, O'Sullivan BP, Robinson KA, et al. Am J Respir Crit Care Med. 2007;176:957-69.

3. Bilton D, Canny G, Conway S, et al. J Cyst Fibros. 2011;10(S2):S79-81.

4. Kraynack NC, McBride JT. Semin Respir Crit Care Med. 2009;30:547-58.

5. Kraynack NC, Gothard MD, Falletta LM, et al. Pediatr Pulmonol. 2011 Apr 4. doi: 10.1002/ppul.21442.

6. Flume PA, Mogayzel PJ Jr, Robinson KA, et al. Am J Respir Crit Care Med. 2009;180:802-8.

7. Parkins MD, Elborn JS. J Antimicrob Chemother. 2010;65:1853-61.

8. Foweraker JE, Laughton CR, Brown DF, et al. J Antimicrob Chemother. 2005;55:921-7.

9. Aaron SD, Vandemheen KL, Ferris W, et al. Lancet. 2005;366:463-71.

10. Bell SC, Bowerman AM, Nixon LE, et al. Eur J Clin Invest. 2000;30:553-9.

11. VanDevanter D, O'Riordan MA, Blumer JL, et al. Respir Res. 2010;11:137.

12. Tunney MM, Klem ER, Fodor AA, et al. Thorax. 2011;66:579-84. 


\title{
S10.2 \\ MAXIMIZING BENEFIT/MINIMIZING RISK: PK-PD CONSIDERATIONS OF ANTIPSEUDOMONAL ANTIBIOTICS
}

\author{
David C. Young, Pharm.D. \\ 1. Intermountain CF Adult Ctr., Univ. of Utah Hospitals \& Clinics, Salt Lake City, UT, USA; 2. Pharmacotherapy, \\ Univ. of Utah College of Pharmacy, Salt Lake City, UT, USA
}

Beta-lactam antibiotics exhibit time-dependent bactericidal activity (1). More importantly, beta-lactam antibiotics exert maximum bactericidal activity when their concentrations are 3-4 times the minimum inhibitory concentration (MIC) (1). In addition, beta-lactam concentrations must remain above the $\mathrm{MIC}$ for $>70 \%$ of the dosing interval in order to achieve maximal bactericidal activity (2). Vogelman et al. reported that the efficacy of ticarcillin against $P$. aeruginosa reached its maximal benefit as the time above MIC approached $100 \%$ (3). This is of particular concern because patients with cystic fibrosis (CF) exhibit increased renal clearance of beta-lactam antibiotics, such as ticarcillin, by as much as $30 \%$ compared to healthy volunteers (4). Our preliminary data demonstrated beta-lactam clearance in $\mathrm{CF}$ patients increases by as much as 23 to $56 \%$ during a 14-day hospital stay (5). Intermittent administration of ticarcillin/clavulanate in patients with $\mathrm{CF}$ resulted in T>MIC against $P$. aeruginos $a$ at a susceptible MIC of 64 $\mu \mathrm{g} / \mathrm{ml}$ of merely $17 \%$ (in press). As a result, patients with $\mathrm{CF}$ require higher doses or more frequent administration of beta-lactam antibiotics (4).

In contrast, continuous infusion (CI) of beta-lactam antibiotics maintains serum levels above MIC at concentrations that maximize bactericidal activity (1). In patients with $\mathrm{CF}$, Rappaz et al. reported that the ceftazidime concentrations delivered via CI exceeded an MIC of $4 \mu \mathrm{g} / \mathrm{ml}$ against $P$. aeruginosa $100 \%$ of the time (6). Hann et al. concluded that CI of cefepime in patients with CF effectively achieves and maintains concentrations above MIC and reduces the total daily dose by $20 \%$ (7). Kuti et al., demonstrated that meropenem delivered via $\mathrm{CI}$ was able to maintain serum concentrations $>4$ $\mu \mathrm{g} / \mathrm{ml}$ for $100 \%$ of the dosing interval (8). While there is sufficient evidence to conclude that $\mathrm{CI}$ is more effective at increasing $\mathrm{T}>\mathrm{MIC}$ for any infecting organism, there is limited data regarding the efficacy of continuous versus intermittent delivery of beta-lactam antibiotics. In the largest published trial to date, Hubert et al. compared ceftazidime delivered via CI versus thrice daily infusion (9). The authors concluded that the regimens were equivalent. However, the mean change in FEV1\% predicted was significantly better in patients with resistant bacteria when ceftazidime was delivered via CI vs. intermittent infusion $(p<0.05)(9)$. Also, the mean difference in time to next intravenous antibiotic course was 0.4 months longer with continuous versus short infusion ceftazidime $(\mathrm{p}=0.04)$. Beta-lactam antibiotics delivered via CI are well tolerated. No difference in laboratory values (i.e. serum creatinine or liver function tests) was seen between ceftazidime delivered via CI versus intermittent infusion $(6,9,10)$. In addition, cefepime and meropenem administered via CI are well tolerated $(8,11)$.

Aminoglycosides, such as tobramycin, exhibit concentration-dependent bactericidal activity (12). As a result, the bacterial killing effect is correlated with peak serum concentrations. Maximal bactericidal activity has been demonstrated when the aminoglycoside peak serum concentration/minimum inhibitory concentration (MIC) ratio is greater than 10 (13). Once-daily dosing can achieve higher peak serum concentrations and peak/MIC ratios and minimize the development of adaptive resistance $(1,14)$. Once daily dosing of aminoglycosides may also reduce the risk of nephrotoxicity $(15,16)$. This has led the CFF to recommend once-daily dosing of aminoglycosides to be the preferred method of administration in all CF patients with acute pulmonary disease (17). However, limitations exist with the use of once-daily dosing of aminoglycosides in CF. The optimal peak serum concentration and peak/MIC ratios have not been established for acute exacerbations of $\mathrm{CF}$ (17). The potential for nephrotoxicity, ototoxicity, and vestibular toxicity necessitate appropriate therapeutic drug level monitoring $(15,18)$. Finally, data regarding efficacy of once versus twice/three times-daily dosing of aminoglycosides is limited. The largest study published to date regarding the optimal aminoglycoside dosing strategy in CF patients (pediatric and adult) was the TOPIC trial (16). The authors concluded that oncedaily administration of tobramycin was equivalent to thrice-daily dosing and resulted in less nephrotoxicity in children.

References: 1. Burgess DS. Use of pharmacokinetics and pharmacodynamics to optimize antimicrobial treatment of Pseudomonas aeruginosa infections. Clin Infect Dis. 2005;40(Suppl 2):S99-104.

2. Burgess DS, Hastings RW, Hardin TC. Pharmacokinetics and pharmacodynamics of cefepime administered by intermittent and continuous infusion. Clin Ther. 2000;22:66-75.

3. Vogelman B, Gudmundsson S, Leggett J, et al. Correlation of antimicrobial pharmacokinetic parameters with therapeutic efficacy in an animal model. J Infect Dis. 1988;158:831-47.

4. Rey E, Treluyer JM, Pons G. Drug disposition in cystic fibrosis. Clin Pharmacokinet. 1998;35:313-29. 
5. Alexander DP. Enhanced beta-lactam elimination during treatment of an acute exacerbation in cystic fibrosis (CF). Pediatr Pulmonol. 2005;Suppl 28:288.

6. Rappaz I, Decosterd LA, Bille J, et al. Continuous infusion of ceftazidime with a portable pump is as effective as thrice-a-day bolus in cystic fibrosis children. Eur J Pediatr. 2000;159:919-25.

7. Han EE, Beringer PM, Falck P, et al. Pilot study of continuous infusion cefepime in adult patients with cystic fibrosis. J Antimicrob Chemother. 2006;57:1017-9.

8. Kuti JL, Nightingale CH, Knauft RF, et al. Pharmacokinetic properties and stability of continuous-infusion meropenem in adults with cystic fibrosis. Clin Ther. 2004;26:493-501.

9. Hubert D, Le Roux E, Lavrut T, et al. Continuous versus intermittent infusions of ceftazidime for treating exacerbation of cystic fibrosis. Antimicrob Agents Chemother. 2009;53:3650-6.

10. Riethmueller J, Junge S, Schroeter TW, et al. Continuous vs thrice-daily ceftazidime for elective intravenous antipseudomonal therapy in cystic fibrosis. Infection. 2009;37:418-23.

11. Bernard E, Breilh D, Bru JP, et al. Is there a rationale for the continuous infusion of cefepime? A multidisciplinary approach. Clin Microbiol Infect. 2003;9:339-48.
12. Edson RS, Terrell CL. The aminoglycosides. Mayo Clin Proc. 1999;74:519-28.

13. Moore RD, Smith CR, Lietman PS. Association of aminoglycoside plasma levels with therapeutic outcome in gram-negative pneumonia. Am J Med. 1984;77:657-62.

14. Barclay ML, Begg EJ, Chambers ST, et al. Adaptive resistance to tobramycin in Pseudomonas aeruginosa lung infection in cystic fibrosis. J Antimicrob Chemother. 1996;37:1155-64.

15. Prayle A, Smyth AR. Aminoglycoside use in cystic fibrosis: therapeutic strategies and toxicity. Curr Opin Pulm Med. 2010;16:604-10.

16. Smyth A, Tan KH, Hyman-Taylor P, et al. Once versus three-times daily regimens of tobramycin treatment for pulmonary exacerbations of cystic fibrosis- the TOPIC study: a randomised controlled trial. Lancet. 2005;365:573-8.

17. Flume PA, Mogayzel PJ, Jr., Robinson KA, et al. Cystic fibrosis pulmonary guidelines: treatment of pulmonary exacerbations. Am J Respir Crit Care Med. 2009; 180:802-8.

18. Begg EJ, Barclay ML, Duffull SB. A suggested approach to once-daily aminoglycoside dosing. Br J Clin Pharmacol. 1995;39:605-9.

\title{
S10.3 \\ OPTIMIZING ANTIBIOTIC DELIVERY: HOME VS. HOSPITAL AND ROUTE OF DELIVERY
}

\author{
Peter J. Mogayzel, Jr., M.D., Ph.D. \\ Dept. of Pediatrics, The Johns Hopkins Hospital, Baltimore, MD, USA
}

Pulmonary exacerbations are a common complication of cystic fibrosis (CF) lung disease, which lead to significant morbidity and accelerate lung function decline $(1,2)$. Despite aggressive therapy, many patients do not return to their pre-exacerbation lung function after treatment (3-5). Therefore, developing the best possible approach to therapy is of vital importance. Unfortunately, systematic review of exacerbation therapy has not provided answers to several important questions regarding the optimal approach to therapy (6). Treatment with antibiotics is the mainstay of exacerbation therapy. Oral antibiotics can be effective in treating the majority of exacerbations (7). However, patients with more severe exacerbations or recurrent symptoms require intravenous (IV) antibiotic therapy.

There is controversy regarding the optimal method for delivery of IV antibiotics. Traditionally, admission to the hospital has been the preferred approach for the administration of IV antibiotics. However, advances in the percutaneuos placement of central venous catheters allow for the delivery of IV antibiotics in an outpatient setting. Systematic review suggests that the use of a longer central line may provide benefit over shorter IV catheters for antibiotic therapy (8). Peripheral insertion of central catheters has gained widespread use throughout the world for the delivery of antibiotics to patients with CF. However, there are reports of venous thrombosis associated with the use of these catheters (9-12). Although totally implantable vascular access devices are frequently used in patients with CF there are no randomized studies about the value of these catheters (13). However, their widespread use suggests that they are safe and effective $(14,15)$. Factors that influence the decision to place an implantable catheter include: frequency of exacerbations, difficulty of placement of a peripherally inserted central catheter and patient preferences. Ultimately, the decision about the type of venous access used should be made on an individual patient basis.

The ability to provide stable access for the delivery of IV antibiotics has allowed patients to receive antibiotic therapy in an outpatient setting. However, there is concern among many care providers that therapy delivered at home will not 
result in comparable outcomes to treatment in the hospital. It is important to remember that the delivery of antibiotics is only one component of the overall therapy of pulmonary exacerbations. Only one study has compared home and hospital treatment of pulmonary exacerbations in a prospective fashion $(6,16)$. Wolter et al $(17)$ found similar outcomes between those patients that completed most of their therapy at home compared to those that remained in the hospital for their entire treatment course. However, several retrospective studies have found that patients treated in a hospitalized setting do in fact have better outcomes $(18,19)$. More recently, Collaco et al (3) examined the outcomes of 1,278 exacerbations experienced by 479 subjects in the US twin and sibling study. These authors found that the outcome of patients treated entirely in the hospital or entirely at home or in some combination of both locales had similar outcomes with respect to lung function and time to the next exacerbation. Studies have suggested that there can be benefits to different aspects of quality of life measures with either hospitalization or home therapy $(17,20)$. Therefore, consideration of the differing burdens of hospitalization or home IV administration must be taken into account when making treatment decisions. The disruption of home and family life must be weighed against the certainty of increased airway clearance, appropriate rest and adherence to medication delivery schedules. For home IV therapy to be successful, the patient must have the time, resources and family support to administer the antibiotics and provide intensive airway clearance and inhalational therapy.

Many considerations must be taken into account when making the decision on how to treat a patient experiencing a pulmonary exacerbation. Unfortunately, there are few studies to help guide these decisions. The type of venous access and the locale of therapy have the potential to greatly influence the outcome of therapy. Therefore, careful consideration should be given when making these decisions.

References: 1. de Boer K, Vandemheen KL, Tullis E, et al. Exacerbation frequency and clinical outcomes in adult patients with cystic fibrosis. Thorax. 2011 Jun 15. [Epub ahead of print]

2. Sanders DB, Bittner RC, Rosenfeld M, et al. Pulmonary exacerbations are associated with subsequent FEV1 decline in both adults and children with cystic fibrosis. Pediatr Pulmonol. 2011;46:393-400.

3. Collaco JM, Green DM, Cutting GR, et al. Location and duration of treatment of cystic fibrosis respiratory exacerbations do not affect outcomes. Am J Respir Crit Care Med. 2011;182:1137-43.

4. Sanders DB, Bittner RC, Rosenfeld M, et al. Failure to recover to baseline pulmonary function after cystic fibrosis pulmonary exacerbation. Am J Respir Crit Care Med. 2010;182:627-32.

5. Sanders DB, Hoffman LR, Emerson J, et al. Return of FEV1 after pulmonary exacerbation in children with cystic fibrosis. Pediatr Pulmonol. 2010;45:127-34.
6. Flume PA, Mogayzel PJ Jr, Robinson KA, et al. Cystic fibrosis pulmonary guidelines: treatment of pulmonary exacerbations. Am J Respir Crit Care Med. 2009; 180:802-8.

7. Briggs EC, Nguyen T, Wall MC, et al. Oral antimicrobial use in outpatient cystic fibrosis pulmonary exacerbation management; a single center experience. Clin Respir J. 2011 May 19. doi: 10.1111/j.1752699X.2011.00246.x. [Epub ahead of print]

8. Prayle AP, Hurley MN, Smyth AR. Percutaneous lines for delivering intravenous antibiotics in people with CF. Cochrane database of systematic reviews (Online) (11):CD008243.

9. Barker M, Thoenes D, Dohmen H, et al. Prevalence of thrombophilia and catheter-related thrombosis in cystic fibrosis. Pediatr Pulmonol. 2005;39:156-61.

10. Bui S, Babre F, Hauchecorne S, et al. Intravenous peripherally-inserted central catheters for antibiotic therapy in children with cystic fibrosis. J Cyst Fibros. 2009;8:326-31.

11. Nash EF, Helm EJ, Stephenson A, et al. Incidence of deep vein thrombosis associated with peripherally inserted central catheters in adults with cystic fibrosis. J Vasc Interv Radiol. 2009;20:347-51.

12. Tolomeo C, Mackey W. Peripherally inserted central catheters (PICCs) in the CF population: one center's experience. Pediatr Nurs. 2003;29:355-9.

13. A-Rahman A, Spencer D. Totally implantable vascular access devices for cystic fibrosis. Cochrane database of systematic reviews (Online) 2003(3):CD004111.

14. Royle TJ, Davies RE, Gannon MX. Totally implantable venous access devices - 20 years' experience of implantation in cystic fibrosis patients. Ann R Coll Surg Engl. 2008;90:679-84.

15. Dal Molin A, Gatta C, Festini F. Management of totally implantable vascular access devices in patients with cystic fibrosis. Minerva Pediatr. 2009;61:549-55.

16. Balaguer A, Gonzalez de Dios J. Home intravenous antibiotics for cystic fibrosis. Cochrane database of systematic reviews (Online) 2008(3):CD001917.

17. Wolter JM, Bowler SD, Nolan PJ, et al. Home intravenous therapy in cystic fibrosis: a prospective randomized trial examining clinical, quality of life and cost aspects. Eur Respir J. 1997;10:896-900.

18. Nazer D, Abdulhamid I, Thomas R, et al. Home versus hospital intravenous antibiotic therapy for acute pulmonary exacerbations in children with cystic fibrosis. Pediatr Pulmonol. 2006;41:744-9.

19. Termoz A, Touzet S, Bourdy S, et al. Effectiveness of home treatment for patients with cystic fibrosis: the intravenous administration of antibiotics to treat respiratory infections. Pediatr Pulmonol. 2008;43:908-15.

20. Yi MS, Tsevat J, Wilmott RW, et al. The impact of treatment of pulmonary exacerbations on the health-related quality of life of patients with cystic fibrosis: does hospitalization make a difference? J Pediatr. 2004;144:711-8. 


\title{
S10.4 \\ TREATING PULMONARY EXACERBATIONS UNDER SPECIAL CIRCUMSTANCES
}

\author{
Elizabeth Tullis, M.D., FRCP(C) \\ Adult CF Program, St. Michael's Hospital, Toronto, ON, Canada
}

Studies of management of patients with CF are limited by the relatively small patient population and heterogeneity of the patient population. Although there is evidence from Phase 3 trials and consensus statements to guide chronic therapies in CF (1-4), the management of pulmonary exacerbations is not as well studied. Consensus statement for management of pulmonary exacerbations was hampered by lack of data, poor quality or conflicting data (5). CF physicians also face the challenge of managing individual patients where specific clinical issues complicate therapy; such as pregnancy, drug allergies or where unusual or multiple bacteria are present. In these situations, where the literature is not very helpful, one usually contacts CF colleagues to ask for advice. This session aims to review an approach to these problems.

With improving survival and increasing numbers of adults with $\mathrm{CF}$, care centres are managing more and more pregnancies. Large registry (6) and single centre studies $(7,8)$ have demonstrated that pregnancy itself does not have an impact on survival. However, clinicians need to be able to manage pulmonary exacerbations in pregnant women with CF. Pregnancy is an exclusion criteria for drug trials and animal teratogenicity studies are difficult to extrapolate, so product monographs are usually not helpful. The usual statement that use in pregnancy is not recommended unless the potential benefits to the mother outweigh the potential risks to the fetus may be the case when treating a woman with a CF pulmonary exacerbation.

There are some drugs that should be avoided in pregnancy including aminoglycosides, tetracyclines and chloramphenicol. Aminoglycosides cross the placenta and there are case reports of fetal ototoxicity including deafness. Clinical stability is an important goal during pregnancy and inhaled tobramycin reduces the number of pulmonary exacerbations. Median levels of tobramycin one hour after receiving $300 \mathrm{mg}$ of tobramycin solution for inhalation are $0.94 \mu \mathrm{g} / \mathrm{ml}$ (range of 0.18 - 03.62) (9). Serum levels after lower doses of inhaled tobramycin can be measured. Tetracyclines may be used to treat $B$ cepacia complex. Tetracyclines cross the placenta and cause yellow or grey staining of deciduous teeth in children born to mothers who received tetracycline in the $2^{\text {nd }}$ and $3^{\text {rd }}$ trimester. No increased incidence of malformations was seen in 2 cohort studies of 274 infants whose mothers used tetracycline in the $1^{\text {st }}$ trimester $(10,11)$. Tetracyclines deposit in the skeleton where they inhibit bone elongation and calcium uptake.
This appears to be a temporary inhibition of bone growth. Trimethoprim-sulfamethoxazole may be used to treat Stenotrophomonas and B cepacia complex. Sulfonamides should not be used after 32 weeks of pregnancy as they displace bilirubin from binding to albumin and can cause kernicterus in the newborn. Trimethoprim should not be used in the first trimester as it is a folate antagonist and may result in increased risk of neural tube defects. Cartilage toxicity has been demonstrated in animal studies of quinolones. However, a controlled study prospectively enrolling and following 200 women exposed to fluoroquinolones during pregnancy did not show increased risk of major congenital malformations in the group exposured in the first trimester and no clinically significant musculoskeletal dysfunctions in the children (12). A series of observational cohort studies suggest that azithromycin does not cause an increased risk of congenital abnormalities when used in pregnancy $(10,11,13)$. There is no data available on the use of aztreonam or meropenem in pregnancy. Cephalosporins and penicillins are generally considered safe in pregnancy. Another common challenge is in the CF patient with unusual or multiple bacteria present in sputum cultures. $\mathrm{CF}$ clinicians are just becoming familiar with treating Stenotrophomonas and Achromobacter, and now we have to decide if we should treat other gram negative bacteria such as Ralstonia, Pandoraea and Inquilinus (14-16). These bacteria are closely related to Burkholderia species but it is not clear if they are pathogens although there are case reports of significant illness in CF patients infected with these bacteria (17). In a similar way, we see nontuberculous mycobacteria (NTM) in combination with the usual $\mathrm{CF}$ pathogens and have to decide if they are bystanders or causing disease. One general approach is to treat the other bacterial pathogens and see if there is a clinical response. If no clinical response occurs, then determining persistence of the NTM with serial cultures and "burden" of infection with smears and using a combination of clinical symptoms and radiographic information (HRCT scan) may help with this decision. A trial of therapy, with frequent reevaluation, may be the best way of dealing with this uncertainty.

When approaching the challenge of making decisions and managing patients in the absence of evidence based guidelines, it is helpful to collect all the information you have and utilize resources. In our centre, the CF physicians get together on a regular basis to discuss the patients with challenging medical problems and put 
together a plan for care. Pulling together a case presentation with a literature review to present to your colleagues followed by a thoughtful discussion and involvement of other experts such as pharmacy is extremely helpful. Of course, email communication with $\mathrm{CF}$ experts around the world identified through internet and a literature search is not only helpful but can be reassuring to know that you are not alone in handling these challenges.

References: 1. Fuchs HJ, Borowitz DS, Christiansen $\mathrm{DH}$, et al. Effect of aerosolized recombinant human DNase on exacerbations of respiratory symptoms and on pulmonary function in patients with cystic fibrosis. The Pulmozyme Study Group. N Engl J Med. 1994;8;331:637-42.

2. Ramsey BW, Pepe MS, Quan JM, et al. Intermittent administration of inhaled tobramycin in patients with cystic fibrosis. Cystic Fibrosis Inhaled Tobramycin Study Group. N Engl J Med. 1999;340:23-30.

3. Elkins MR, Robinson M, Rose BR, et al. A controlled trial of long-term inhaled hypertonic saline in patients with cystic fibrosis. $N$ Engl J Med. 2006;354:229-40.

4. Saiman L, Marshall BC, Mayer-Hamblett N, et al. Azithromycin in patients with cystic fibrosis chronically infected with $P$. aeruginosa: a randomized controlled trial. JAMA. 2003;290:1749-56.

5. Flume PA, Mogayzel PJ Jr, Robinson KA, et al. Cystic fibrosis pulmonary guidelines: treatment of pulmonary exacerbations. Am J Resp Crit Care Med. 2009;180:802-8.

6. Goss CH, Rubenfeld GD, Otto K, et al. The effect of pregnancy on survival in women with cystic fibrosis. Chest. 2003;124:1460-8.
7. Gilljam M, Antoniou M, Shin J, et al. Pregnancy in cystic fibrosis. Fetal and maternal outcome. Chest. 2000;118:85-91.

8. Cheng EY, Goss CH, McKone EF, et al. Aggressive prenatal care results in successful fetal outcomes in $\mathrm{CF}$ women. J Cyst Fibros. 2006;5:85-91.

9. TOBI monograph.

10. Aselton P, Jick H, Milunsky A, et al. Firsttrimester drug use and congenital disorders. Obstet Gynecol. 1985;65:451-5.

11. Jick H, Holmes LB, Hunter JR, et al. First-trimester drug use and congenital disorders. JAMA. 1981;246:343-6.

12. Loebstein R, Addis A, Ho E, et al. Pregnancy outcome following gestational exposure to fluoroquinolones: a multicenter prospective controlled study. Antimicrob Agents Chemother. 1998;42:1336-9.

13. McCormack WM, Rosner B, Lee YH, et al. Effect on birth weight of erythromycin treatment of pregnant women. Obstet Gynecol. 1987;69:202-7.

14. Coenye T, Vandamme P, LiPuma JJ. Infection by Ralstonia species in cystic fibrosis patients: identification of R. pickettii and R. mannitolilytica by polymerase chain reaction. Emerg Infect Dis. 2002;8:692-6.

15. Coenye T, Vandamme P, LiPuma JJ. Ralstonia respiraculi sp. nov., isolated from the respiratory tract of cystic fibrosis patients. Int J Syst Evol Microbiol. 2003;53:1339-42.

16. Cooke RP, O'Neill WA, Xu J, et al. Inquilinus limosus isolated from a cystic fibrosis patient: first UK report. Br J Biomed Sci. 2007;64:127-9.

17. Jorgensen IM, Johansen HK, Frederiksen B, et al. Epidemic spread of Pandoraea apista, a new pathogen causing severe lung disease in cystic fibrosis patients. Pediatr Pulmonol. 2003;36:439-46.

\title{
S11.2 ASSESSMENT OF NEUROPSYCHOLOGICAL FUNCTIONING
}

\author{
Cara I. Kimberg, Ph.D. \\ St. Jude Children's Research Hospital, Memphis, TN, USA
}

It is generally accepted that cystic fibrosis (CF) does not impact cognitive function; however, this is substantiated primarily through clinical observations and there are few empirical studies to support this claim. It is important that the cognitive skills of individuals with CF are better understood on a population level, as even subtle deficits may impact disease management. Based on the similarities between CF and other disease groups, there is reason to believe that individuals with $\mathrm{CF}$ may be at-risk for attentional deficits or executive dysfunction (i.e., impairments in planning, organization, shifting, initiating, and emotional control). For example, individuals with sleep apnea, a disorder characterized by pauses in breathing or shallow breaths, are found to demonstrate executive dysfunction and behaviors consistent with attention deficit hyperactivity disorder (ADHD)(1). Of note, when sleep apnea is treated, these neurobehavioral deficits remit. Similarly, patients with chronic obstructive pulmonary disease (COPD) performed worse on tests of attention, executive function (EF) and processing speed compared to controls. The degree of impairment was predicted by low levels of forced expiratory volume (FEV) and forced vital capacity $(\mathrm{FVC})(2,3)$. Using the negative cognitive effects of chronic or intermittent hypoxia demonstrated by patients with sleep apnea and COPD as a model, it is plausible that, due to compromised pulmonary functioning, individuals with $\mathrm{CF}$ may demonstrate a similar pattern of deficits. This may be especially true for pediatric patients, as oxygen deprivation could 
have more adverse consequences on the developing brain.

To date, limited research has been conducted on the neurocognitive function of individuals with CF. A study by Zinman and colleagues (4) explored the differential impact of oxygen versus room air at night on cognitive function and found no group differences in processing speed, memory or achievement. Koscik and colleagues (5) determined that children with vitamin E deficiency at traditional diagnosis had poorer cognitive performance on a task of general intellectual ability several years later compared to children diagnosed through newborn screening who did not demonstrate this nutritional deficit. Lastly, Dobbin and colleagues (6) evaluated the impact of pulmonary exacerbations on objective neurobehavioral performance. Pulmonary exacerbations were found to negatively impact neurocognitive performance, as well as a driving simulation task. Importantly, treatment of the exacerbation led to improved cognitive function.

The value of understanding the cognitive skills, particularly in the domains of attention and EF, of individuals with $\mathrm{CF}$ is highlighted through intervention research. For example, in the context of an adherence intervention, it is possible that responders and nonresponders differ based on EF skills. An intervention that utilizes text messages to remind patients to take their medication may be successful for adolescents with executive dysfunction, as the text message directly targets their weakness (i.e., poor organization and planning). In contrast, this intervention may have no effect on adolescents with good EF, as the text message does not address the underlying reason for poor adherence (e.g., depressive symptoms). Thus, the success of an intervention may be influenced by the cognitive profile of the participants. It is important for researchers to be mindful of cognitive function as a potentially confounding factor when designing and implementing interventions. Clinically, it is essential for multidisciplinary CF team members to be aware of individual cognitive impairments. According to the Diagnostic and Statistical Manual of Mental Disorders (DSM-IV-TR, ref 7), 3\%-7\% of school-age children are diagnosed with ADHD; therefore, it would be expected that a subset of children with $\mathrm{CF}$ also meet criteria for this diagnosis. In fact, a retrospective study by Georgiopoulos and Hua (8) determined that $9.6 \%$ of children and adolescents with CF, who were seen by the Massachusetts General Hospital CF Program, were referred to a psychiatrist and subsequently received a diagnosis of ADHD. The behavioral profile of ADHD, which includes symptoms of impulsivity, inattention, hyperactivity and executive dysfunction, has the potential to significantly complicate disease management if not properly treated. For example, an impulsive child may eat without taking enzymes or an adult with poor working memory skills may forget the order in which to complete his/her treatments. These instances of poor disease management are consistent with the Georgiopoulos and Hua study (8), as many of the patients ultimately diagnosed with ADHD presented with poor treatment adherence.

To evaluate attention and EF, standardized measures including performance-based tests and questionnaires can be administered. Such measures are given as part of a clinical or research neuropsychological assessment. Neuropsychological evaluations focus on the link between brain structures/pathways and cognitive skills. Test measures for the assessment are selected based on the referral or research question and evaluate general intellectual function, academic achievement, language, visual-spatial and visual-motor abilities, attention, learning and memory, EF and behavioral/emotional functioning. A performance profile, based on the determined pattern of strengths and weaknesses across the multiple domains, is used to guide recommendations. In sum, there are identified factors that may place individuals with $\mathrm{CF}$ at risk for attentional and EF impairment. Further research, using neuropsychological assessment, is needed to delineate the cognitive profile of these patients, as cognitive function may impact disease management and research findings.

References: 1. Beebe DW, Gozal D. Obstructive sleep apnea and the prefrontal cortex: toward a comprehensive model linking nocturnal upper airway obstruction to daytime cognitive and behavioral deficits. J Sleep Res. 2002;11:1-16.

2. Areza-Fegyveres R, Kairalla RA, Carvalho CRR, et al. Cognition and chronic hypoxia in pulmonary diseases. Dementia Neuropsychol. 2010;4:14-22.

3. Bass JL, Corwin M, Gozal D, et al. The effect of chronic or intermittent hypoxia on cognition in childhood: A review of the evidence. Pediatrics. 2004;114:805-16.

4. Zinman R, Corey M, Coates AL, et al. Nocturnal home oxygen in the treatment of hypoxemia cystic fibrosis patients. J. Pediatr. 1989;114:368-77.

5. Koscik RL, Farrell PM, Kosorok MR, et al. Cognitive function of children with cystic fibrosis: Deleterious effect of early malnutrition. Pediatrics. 2004;113:154958.

6. Dobbin CJ, Bartlett D, Melehan K, et al. The effect of infective exacerbations on sleep and neurobehavioral function in cystic fibrosis. Am J Resp Crit Care Med. 2005;172:99-104.

7. American Psychiatric Association: Diagnostic and Statistical Manual of Mental Disorders, Fourth Edition, Text Revision. Washington, DC: American Psychiatric Association, 2000.

8. Georgiopoulos AM, Hua LL. The diagnosis and treatment of attention deficit-hyperactivity disorder in children and adolescents with cystic fibrosis: A retrospective study. Psychosomatics. 2011;52:160-6. 


\title{
S11.3 \\ PREVALENCE, IMPACT AND TREATMENT OF ADHD IN CYSTIC FIBROSIS
}

\author{
Anna M. Georgiopoulos, M.D. \\ Dept. of Child \& Adolescent Psychiatry, Massachusetts General Hospital/Harvard Med. School, Boston, MA, USA
}

Prevalence of ADHD in pediatric medical illness and CF. Attention Deficit-Hyperactivity Disorder (ADHD) occurs in $10 \%$ of children (1) and $4-5 \%$ of adults (2) in the general population. Chronic medical illness increases risk for psychiatric illness (3). A study of chronically ill children revealed ADHD in $9.2 \%$ vs. $4.3 \%$ in controls (4). While ADHD is strongly familial (5), medical stressors such as intrauterine growth retardation (6), perinatal hypoxia (7), and malnutrition (8) could encourage phenotypic expression in genetically predisposed patients. The risk of ADHD is higher in males with congenital adrenal hyperplasia (18.2\%) and familial male precocious puberty (44.4\%) (9). ADHD rates of $38 \%-$ $50 \%+$ occur in fetal alcohol syndromes (10), congenital heart disease (11), chromosome 18 abnormalities (12), and velocardiofacial syndrome (13). In a cohort of 188 pediatric CF outpatients, ADHD was diagnosed by a child psychiatrist in $9.6 \%$; $59 \%$ had a family history of ADHD (14). These data may underestimate the prevalence of $\mathrm{ADHD}$ in $\mathrm{CF}$, as they exclude those declining evaluation or diagnosed elsewhere. In another study of $\mathrm{CF}$ children, $21 \%$ met criteria for a disruptive behavior disorder (15). By comparison, the rate of ADHD in diabetic children $(2.1 \%)$ was lower than in complicated epilepsy (12.0\%) (16).

Prevalence of ADHD in adults with $\mathrm{CF}$ and pediatric patient caregivers. The Adult ADHD Scale Self Report Symptom Checklist v1.1 (ASRS) was administered to adult $\mathrm{CF}$ outpatients and caregivers of pediatric CF patients as part of an ongoing study of psychopathology in CF. Mean age of adult CF patients (49\% male) was $34.6 \pm 11.3$, with mean FEV1 of $60.3 \pm 22.4$ and BMI of $23.9 \pm 3.8$. The mean ASRS 18-item total was $25.4 \pm 10.1$ in adult CF patients and $29.4 \pm 8.0$ in caregivers. Prevalence data is shown in the Table below.

Effects on treatment adherence and medical outcomes. ADHD is associated with functional impairment including in self care $(2,17)$ and substance abuse (18). Longitudinal data links impulsivity with early mortality (19). Task avoidance, inattention and difficulty following directions may interfere with health regimens. Oppositionality, forgetting, and trouble managing the time required for care routines are commonly reported in CF families $(20,21)$. White et al. (15) identified a trend between externalizing disorders and lower CF adherence.

In our study of pediatric $\mathrm{CF}$ patients with ADHD (14), 61\% (11 of 18) presented with CF non-adherence. The 3 who refused ADHD treatment and the 2 who had medication trials resulting in minimal or no improve- ment had no change in adherence. One had much improved ADHD symptoms after treatment, but minimal improvement in adherence. The remaining 5 demonstrated improvement in adherence after successful ADHD treatment.

In our adult $\mathrm{CF}$ pilot sample described above, total score on the CF Questionnaire-Revised (CFQ-R) was higher $(\mathrm{p}=0.035)$ in patients meeting criteria for probable ADHD $(47.7 \pm 9.1)$ vs. those without ADHD

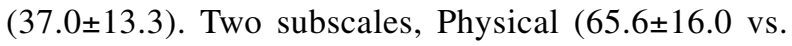
$45.0 \pm 26.7 ; \mathrm{p}=0.04)$ and Vitality $(58.3 \pm 19.1$ vs. $44.8 \pm 20.9 ; \mathrm{p}=0.09$ ) accounted for this difference. FEV1 and BMI did not differ significantly in the ADHD vs. non-ADHD groups. There was significantly less self-reported adherence to exercise, nasal irrigation and diabetes care $(\mathrm{p}<0.05)$ in $\mathrm{CF}$ patients with any psychopathology (depression, anxiety and/or ADHD) than with no psychopathology. However, there were no significant differences in self-reported adherence between CF adults with ADHD vs. no ADHD. Several limitations complicate this finding, including sample size; the high prevalence of depression/anxiety in the non-ADHD group; sample bias toward CF-ADHD patients who do attend clinic and are more likely to adhere to care; and lack of insight/decreased accuracy in self-reported adherence in patients with ADHD.

Treatment of ADHD in CF. In our pediatric cohort (14), ADHD medication treatment was attempted in 13 cases, achieving an improvement rating of much or very much improved in 8 cases. In 3 cases, the Best Regimen consisted of stimulant monotherapy (mixed amphetamine salts; methylphenidate); 2 consisted of non-stimulant monotherapy (atomoxetine; nortriptyline); 2 used a combination of 2 non-stimulants (atomoxetine or nortriptyline + guanfacine); and 1 used a stimulant plus a non-stimulant (buproprion XL+ Orosmethylphenidate). The decision about whether and how to treat ADHD in CF requires careful consideration of psychiatric and medical status. In our study, stimulants were more effective but more often associated with weight loss than non-stimulants. The 2 patients treated with nortriptyline demonstrated increases in BMI percentile (from $22 \%$ to $63 \%$ and from $34 \%$ to $60 \%$ ) along with improvement in ADHD. Tricyclic antidepressants are considered third-line ADHD agents, after stimulants and FDA-approved nonstimulants. However, the risk/benefit ratio may differ in $\mathrm{CF}$, where low weight and comorbid anxiety 
and depression are common. If stimulants are used, boosting caloric intake, adding cyproheptadine or other appetite stimulators and treating constipation may help maintain weight.

Conclusion. ADHD is common and treatable in patients with CF. Oppositional behaviors, distractibility, forgetfulness or extreme motor activity interfering with $\mathrm{CF}$ care should trigger consideration of ADHD. Stimulants, nonstimulants and combination therapies are viable treatment options. Further research on the prevalence and treatment of ADHD in CF and its impact on medical adherence and outcomes is warranted.

References: 1. Faraone SV, et al. The worldwide prevalence of ADHD: is it an American condition? World Psychiatry. 2003;2:104-13.

2. Kessler RC, et al. The prevalence and correlates of adult ADHD in the U.S.: results from the National Comorbidity Survey Replication. Am J Psychiatry. 2006;163:716-23.

3. Wells KB, et al. Psychiatric disorder in a sample of the general population with and without chronic medical conditions. Am J Psychiatry. 1988;145:976-81.

4. Cadman D, et al. Chronic illness, disability and mental and social well-being: Findings of the Ontario Child Health Study. Pediatrics. 1987;79:805-13.

5 . Faraone SV. Genetics of adult attention-deficit/hyperactivity disorder. Psychiatr Clin North Am. 2004;27:303-21.

6 . Heinonen $\mathrm{K}$, et al. Behavioural symptoms of attention deficit/hyperactivity disorder in preterm and term children born small and appropriate for gestational age: a longitudinal study. BMC Pediatr. 2010;10:91.

7. Boksa P, et al. Birth insult interacts with stress at adulthood to alter dopaminergic function in animal models: possible implications for schizophrenia and other disorders. Neurosci Biobehav Rev. 2003;27:91-101.

8. Galler JR, et al. A follow-up study of the influence of early malnutrition on development: behavior at home and at school. J Am Acad Child Adolesc Psychiatry. 1989;28:254-61.

9. Mueller SC, et al. Psychiatric characterization of children with genetic causes of hyperandrogenism. Eur J Endocrinol. 2010;163:801-10.

10. Peadon E, et al. Distinguishing between attentiondeficit hyperactivity and fetal alcohol spectrum disorders in children: clinical guidelines. Neuropsychiatr Dis Treat. 2010;6:509-15.

11. Vetter VL, et al. Cardiovascular monitoring of children and adolescents with heart disease receiving medications for attention deficit/hyperactivity disorder [corrected]: a scientific statement from the American Heart Association Council on Cardiovascular Disease in the Young Congenital Cardiac Defects Committee and the Council on Cardiovascular Nursing. Circulation. 2008;117:2407-23.

12. Zavala J, et al. Psychiatric syndromes in individuals with chromosome 18 abnormalities. Am J Med Genet B Neuropsychiatr Genet. 2010;153B:83745.

13. Jolin EM, et al. Affective disorders and other psychiatric diagnoses in children and adolescents with $22 \mathrm{q} 11.2$ Deletion Syndrome. J Affect Disord. 2009;119:177-80.

14. Georgiopoulos AM, et al. The diagnosis and treatment of attention deficit-hyperactivity disorder in children and adolescents with CF: a retrospective study. Psychosomatics. 2011;52:160-6.

15. White T, et al. Adherence and psychopathology in children and adolescents with CF. Eur Child Adolesc Psychiatry. 2009;18:96-104.

16. Davies $S$, et al. A population survey of mental health problems in children with epilepsy. Dev Med Child Neurol. 2003;45:292-5.

17. Biederman J, et al. Functional impairments in adults with self-reports of diagnosed ADHD: A controlled study of 1001 adults in the community. J Clin Psychiatry. 2006;67:524-40.

18. Biederman J, et al. Psychoactive substance use disorders in adults with attention deficit hyperactivity disorder: effects of ADHD and psychiatric comorbidity. Am J Psychiatry. 1995;152:1652-8.

19. Friedman HS, et al. Psychosocial and behavioral predictors of longevity. The aging and death of the "Termites". Am J Psychol. 1995;50:69-78.

20. Modi AC, et al. Barriers to treatment adherence for children with $\mathrm{CF}$ and asthma: what gets in the way? J Pediatr Psychol. 2006;31:846-58.

21. Dziuban EJ, et al. Identifying barriers to treatment adherence and related attitudinal patterns in adolescents with CF. Pediatr Pulmonol. 2010;45:450-8.

Prevalence of ADHD, Depression, and Anxiety in CF

\begin{tabular}{lcc}
\hline & Adult Patients N=54 & CF Care-givers N=8 \\
& $\mathrm{N}(\%)$ & $5(\%)$ \\
\hline Any psychiatric comorbidity & $27(50)$ & $3(38)$ \\
ADHD (probable ADHD on 6-item ASRS) & $8(15)$ & $3(38)$ \\
Depression (HADS 11+/CESD 16+) & $19(35)$ & $0(0)$ \\
Anxiety (HADS 11+) & $13(25)$ & \\
\hline
\end{tabular}




\title{
S11.4 \\ COGNITIVE BEHAVIORAL THERAPY TO PROMOTE ADHERENCE IN ADHD AND MEDICAL ILLNESS
}

\author{
Bruce J. Masek, Ph.D. \\ Dept. of Child \& Adolescent Psychiatry, Massachusetts General Hospital, Boston, MA, USA
}

\begin{abstract}
Nonadherence with medical treatment regimens is prevalent in an estimated one out of two children with chronic medical illness. The model of adherence risks described by Ickovics and Meisler (1) provides a useful framework for the development of interventions to improve adherence to medical therapy in children with chronic illness. In this model five factors are considered, which include: 1) child's developmental stage; 2) disease symptoms; 3 ) complexity of treatment regimen; 4) child-parent relationship; and 5) clinical setting. Behaviorally-based interventions that address one or more of these factors are generally effective for increasing treatment regimen adherence for childhood illnesses (2). The CF patient with ADHD may present a special challenge to health care providers in terms of nonadherence due to behavioral noncompliance, which is so often a feature of the disorder. CBT approaches
\end{abstract}

focused on skills-based interventions to treat ADHD symptoms in adults have recently gained empirical support (3). The aim of this talk will be to describe CBT interventions to promote medical adherence in children and adult CF patients with ADHD that can be delivered by a member of the multi-disciplinary health care team.

References: 1. Ickovics JR, Meisler AW. Adherence in AIDS clinical trials: A framework for clinical research and clinical care. $\mathrm{J}$ Clin Epidemiol. 1997;50:385-91.

2. Graves MM, Roberts MC, Rapoff M, et al. The efficacy of adherence interventions for chronically ill children: A meta-analytic review. J Ped Psychol. 2010;35:368-82.

3. Knouse LE, Safren SA. Current status of cognitive behavioral therapy for adult attention-deficit hyperactivity disorder. Psychiatr Clin N Am. 2010;33:497-509.

\section{S12.1 \\ IS THERE A PRIMARY NEUTROPHIL DEFECT IN CF?}

\author{
Gregory P. Downey, M.D. \\ 1. Medicine, National Jewish Health, Univ. of Colorado, Denver, CO, USA; 2. Immunology, Univ. of Colorado \\ Denver, Denver, CO, USA
}

Cystic fibrosis $(\mathrm{CF})$ is an autosomal recessive disorder caused by mutations in the cystic fibrosis transmembrane conductance regulator (CFTR), a cAMP regulated $\mathrm{Cl}^{-}$channel $(1,2)$. While $\mathrm{CF}$ is a multi-organ disease, the clinical course of affected individuals is dominated by recurrent respiratory tract infections leading eventually to respiratory failure $(3,4)$. Although the life expectancy of affected individuals has increased remarkably in recent years, the vast majority of $\mathrm{CF}$ patients will die from pulmonary disease consequent to progressive inflammatory damage (4-6). One of the characteristic features of CF is persistent and neutrophil-predominant inflammation involving the lower respiratory tract, initially centered in the airways. In fact, pulmonary inflammation is observed very early in life, even in the absence of detectable infection, suggesting the possibility of a primary abnormality of regulation of inflammation (7). Paradoxically, despite the presence of increased numbers of neutrophils, bacteria survive and often thrive in $\mathrm{CF}$ lungs, suggesting a defect in antimicrobial functions of phagocytes in the CF airway (7-14). Whether this reflects an intrinsic neutrophil defect or is a consequence of the abnormal milieu of the $\mathrm{CF}$ lung remains unre- solved (15). We will review selected aspects of abnormal neutrophil function that have been reported and consider genetic versus inflammatory neutrophil reprogramming in $\mathrm{CF}$.

One possibility is that there is an intrinsic defect in phagocyte function. Indeed, there are reports of defects in CF neutrophils, including increased oxidant production (16), decreased shedding of L-selectin (17), differential expression of TLRs (18), and alterations in $\mathrm{Cl}^{-}$flux and intracellular $\mathrm{pH}$ regulation (19) that may hamper antimicrobial functions and predispose to inflammatory tissue injury. However, because expression of CFTR mRNA and protein is normally very low in professional phagocytes such as neutrophils and macrophages (20), it is possible that these reported defects are secondary to extrinsic factors, such as abnormalities in the airway surface liquid (ASL) or to the systemic effects of pulmonary inflammation consequent to infection. However, some of these abnormalities in neutrophil function have been detected in asymptomatic CF carriers who have no apparent indication of systemic or pulmonary inflammation, compatible with the notion of an intrinsic phagocyte defect. 
As noted above, another possibility is that phagocyte dysfunction is secondary to defective epithelial function consequent to CFTR mutations leading to dehydration and increased viscosity of the airway mucous (21), to increased concentrations of proteases such as elastase in ASL leading to cleavage and inactivation of membrane receptors on the phagocytes or degradation of anti-microbial factors in the ASL $(22,23)$, or to abnormal function of epithelial derived antimicrobial factors such as $\beta$ defensins in the ASL (24). An additional possibility is that there is failure to clear effete neutrophils from the $\mathrm{CF}$ lung, either as a consequence of delayed apoptosis of the neutrophils or defective clearance of the apoptotic neutrophils by the airway epithelial cells (25).

From the above discussion it is apparent that defective neutrophil function in CF is likely multifactorial with contributions from primary defects in neutrophil function, from the abnormal composition of the ASL, and from abnormal clearance of apoptotic neutrophils leading to a prolonged and augmented inflammatory response in the CF lung.

References: 1. Lommatzsch ST, Aris R. Genetics of cystic fibrosis. Semin Respir Crit Care Med. 2009;30:531-8.

2. O'Sullivan BP, Freedman SD. Cystic fibrosis. Lancet. 2009;373:1891-904.

3. Davis PB. Cystic fibrosis. Pediatr Rev. 2001;22:257-64.

4. Ratjen FA. Cystic fibrosis: pathogenesis and future treatment strategies. Respir Care. 2009;54:595-605.

5. Corey M, Farewell V. Determinants of mortality from cystic fibrosis in Canada, 1970-1989. Am J Epidemiol. 1996;143:1007-17.

6. Mogayzel PJ, Jr., Flume PA. Update in cystic fibrosis 2009. Am J Respir Crit Care Med. 2010;181:539-44.

7. Khan TZ, Wagener JS, Bost T, et al. Early pulmonary inflammation in infants with cystic fibrosis. Am J Respir Crit Care Med. 1995;151:1075-82.

8. Konstan MW, Hilliard KA, Norvell TM, et al. Bronchoalveolar lavage findings in cystic fibrosis patients with stable, clinically mild lung disease suggest ongoing infection and inflammation. Am J Respir Crit Care Med. 1994;150:448-54.

9. Balough K, McCubbin M, Weinberger M, et al. The relationship between infection and inflammation in the early stages of lung disease from cystic fibrosis. Pediatr Pulmonol. 1995;20:63-70.

10. Noah TL, Black HR, Cheng PW, et al. Nasal and bronchoalveolar lavage fluid cytokines in early cystic fibrosis. J Infect Dis. 1997;175:638-47.

11. Kirchner KK, Wagener JS, Khan TZ, et al. Increased DNA levels in bronchoalveolar lavage fluid obtained from infants with cystic fibrosis. Am J Respir Crit Care Med. 1996;154:1426-9.

12. Armstrong DS, Grimwood K, Carlin JB, et al. Lower airway inflammation in infants and young chil- dren with cystic fibrosis. Am J Respir Crit Care Med. 1997; 156:1197-204.

13. Cantin A. Cystic fibrosis lung inflammation: early, sustained, and severe. Am J Respir Crit Care Med. 1995;151:939-41.

14. Rosenfeld M, Gibson RL, McNamara S, et al. Early pulmonary infection, inflammation, and clinical outcomes in infants with cystic fibrosis. Pediatr Pulmonol. 2001;32:356-66.

15. Moraes TJ, Plumb J, Martin R, et al. Abnormalities in the pulmonary innate immune system in cystic fibrosis. Am J Respir Cell Mol Biol. 2006;34:364-74. PMCID: 2644201.

16. Witko-Sarsat V, Allen RC, Paulais M, et al. Disturbed myeloperoxidase-dependent activity of neutrophils in cystic fibrosis homozygotes and heterozygotes, and its correction by amiloride. J Immunol. 1996;157:2728-35.

17. Russell KJ, McRedmond J, Mukherji N, et al. Neutrophil adhesion molecule surface expression and responsiveness in cystic fibrosis. Am J Respir Crit Care Med. 1998;157:756-61.

18. Petit-Bertron AF, Tabary O, Corvol H, et al. Circulating and airway neutrophils in cystic fibrosis display different TLR expression and responsiveness to interleukin-10. Cytokine. 2008;41:54-60.

19. Coakley RJ, Taggart C, Canny G, et al. Altered intracellular $\mathrm{pH}$ regulation in neutrophils from patients with cystic fibrosis. Am J Physiol Lung Cell Mol Physiol. 2000;279:L66-74.

20. Yoshimura K, Nakamura H, Trapnell BC, et al. Expression of the CFTR gene in cells of non-epithelial origin. Nucleic Acids Res. 1991;19:5417-23.

21. Matsui H, Verghese MW, Kesimer M, et al. Reduced three-dimensional motility in dehydrated airway mucus prevents neutrophil capture and killing bacteria on airway epithelial surfaces. J Immunol. 2005;175:1090-9.

22. Alexis NE, Muhlebach MS, Peden DB, et al. Attenuation of host defense function of lung phagocytes in young cystic fibrosis patients. J Cyst Fibros. 2006;5:17-25. PMCID: 1764441.

23. Vandivier RW, Fadok VA, Hoffmann PR, et al. Elastase-mediated phosphatidylserine receptor cleavage impairs apoptotic cell clearance in cystic fibrosis and bronchiectasis. J Clin Invest. 2002;109:661-70. PMCID: 150889 .

24. Goldman MJ, Anderson GM, Stolzenberg ED, et al. Human beta-defensin-1 is a salt-sensitive antibiotic in lung that is inactivated in cystic fibrosis. Cell. 1997;88:553-60.

25. Vandivier RW, Richens TR, Horstmann SA, et al. Dysfunctional CFTR inhibits phagocytosis of apoptotic cells with proinflammatory consequences. Am J Physiol Lung Cell Mol Physiol. 2009;297:L677-86. PMCID: 2770781. 


\title{
S12.2 \\ ANTIMICROBIALS IN THE CF AIRWAYS - HOW DISEASE ALTERS INNATE IMMUNITY
}

\author{
Paul B. McCray, M.D. \\ Department of Pediatrics, Univ. of Iowa Hospitals \& Clinics, Iowa City, IA, USA
}

Loss of CFTR function has a profound impact on host defense in the respiratory tract, resulting in chronic infection and inflammation. The sequence of events leading from loss of CFTR anion channel function to lung disease pathology is complex, likely multifactorial, and a subject of intense investigation. Thus, no single hypothesis or pathologic scheme may suffice to explain the progressive lung disease associated with CF. Here are briefly summarized some facets of the antimicrobial defenses of the airways and how they may be altered in CF and contribute to lung disease.

Secreted host defense molecules of the airways. The surface and submucosal gland epithelia elaborate a multilayered, constitutive and inducible array of antimicrobials (1). This antimicrobial shield includes a number of peptide, protein, and lipid components. Some examples include the alpha- and beta-defensin peptides, the human cathelicidin peptide LL-37, lysozyme, lactoferrin, SLPI, surfactant proteins A and D, elafin, lipocalin-2, CCL20, S100 family members calgranulin A and B, and PLUNC. In addition to these polypeptides, secreted lipids including cholesteryl esters contribute directly to broad spectrum antimicrobial activity $(2,3)$. A lactoperoxidase based oxidative system also contributes to host defense through the production of the potent antimicrobial hypothiocyanite (4-6). The combined effects of these molecules are to inactivate inhaled or aspirated microbes without stimulating inflammation. Their mechanisms of action are diverse and include nutrient sequestration, opsonization, cell signaling, surfactant/dispersant (7), bacteriocidal, and bacteriostatic activities. Synergy and additivity contribute to the success of this redundant system. In concert, this polyfunctional system is remarkably effective, yet in CF this arm of innate mucosal immunity breaks down.

Loss of CFTR function impairs airway antimicrobial defenses by several mechanisms. CFTR is an anion channel. In addition to playing an important role in regulating the airway surface liquid (ASL) volume and composition through $\mathrm{Cl}^{-}$transport, CFTR also conducts other anions including $\mathrm{HCO}_{3}^{-}, \mathrm{SCN}^{-}$, and glutathione. Therefore impaired CFTR channel activity can have several effects on the ASL environment. Impaired bicarbonate secretion may alter ASL pH (8) and characteristics of secreted mucins $(9,10)$. The combined effect of subtle changes in ASL composition and volume create an unfavorable environment for antimicrobials. Furthermore, changes in mucin composition and viscosity (11) may further hamper their effects by altering mucin charge properties and by reducing mucociliary clearance.
Secretion of antimicrobials by surface and submucosal gland epithelia is both constitutive and responsive to neurohumoral stimuli. CFTR-dependent secretion is defective in $\mathrm{CF}$ and therefore the net abundance of antimicrobials may be reduced $(12,13)$. Early evidence in a porcine model indicates that tracheal submucosal gland tissue mass is reduced in $\mathrm{CF}$, a finding that if confirmed in humans, may provide an additional anatomical contribution to the host defense defect early in life. Loss of CFTR may alter the transcriptional program of cells, resulting in changes in the abundant gene products that contribute to host defense. For example, Erzurum and coworkers noted a reduction in the functional expression of interferon inducible gene products in primary $\mathrm{CF}$ airway epithelia, perhaps contributing to a reduction in antiviral defenses $(14,15)$. Such a change in antiviral immunity could also contribute to inflammation and secondary bacterial colonization (16).

The impact of disease on antimicrobials. In addition to the many direct links between loss of CFTR function and antimicrobial defenses, as the disease progresses and inflammation and infection persist additional secondary effects of disease further compromise airway innate immunity. A number of proteases including neutrophil elastase, cathepsins $\mathrm{S}$ and $\mathrm{G}$, and bacterial products, can directly cleave host defense molecules and further reduce or destroy their activities. Chronic infection and inflammation also trigger extensive remodeling of the epithelium, changing the proportions of cell types present, causing goblet cell metaplasia, and further changing the secretory profile of the cells.

Lessons from new animal models. While mouse models contributed greatly to disease understanding, they fail to develop lung disease similar to humans with CF. Recently, new models of CF were developed using gene targeting in pigs $(17)$ and ferrets $(18,19)$. These new models reproduce several features of $\mathrm{CF}$ disease $(18,20,21)$. At birth, the airways of CFTR targeted pigs are free of inflammation but manifest a host defense defect without the secondary consequences of infection $(17,21)$. Within the first months of life CF pigs spontaneously develop lung disease manifested by infection with bacteria, airway remodeling and mucus hypersecretion. Moreover, after intrapulmonary bacterial challenge with $S$. aureus, CF pigs fail to eradicate bacteria as effectively as wild-type pigs (17). These results suggest that impaired bacterial elimination is the pathogenic event initiating a cascade of inflammation and pathology. CF pigs also exhibit altered tracheal cartilage and luminal narrowing at birth suggesting that the prenatal development 
of the lung may be perturbed (22). Such anatomic abnormalities could further predispose to airway infection by influencing airflow and particle deposition patterns. Adult CF ferrets also develop a lung disease phenotype with similarities to human $\mathrm{CF}$, including bacterial colonization (personal communication, John Engelhardt).

References: 1. Bartlett JA, Fischer AJ, McCray PB Jr. Innate immune functions of the airway epithelium. Contrib Microbiol. 2008;15:147-63.

2. Do TQ, Moshkani S, Castillo P, et al. Lipids including cholesteryl linoleate and cholesteryl arachidonate contribute to the inherent antibacterial activity of human nasal fluid. J Immunol. 2008;181:4177-87.

3. Lee JT, Jansen M, Yilma AN, et al. Antimicrobial lipids: novel innate defense molecules are elevated in sinus secretions of patients with chronic rhinosinusitis. Am J Rhinol Allergy. 2010;24:99-104.

4. Moskwa P, Lorentzen D, Excoffon KJ, et al. A novel host defense system of airways is defective in cystic fibrosis. Am J Respir Crit Care Med. 2007;175:174-83.

5. Conner GE, Wijkstrom-Frei C, Randell SH, et al. The lactoperoxidase system links anion transport to host defense in cystic fibrosis. FEBS Lett. 2007;581:271-8.

6. Pedemonte N, Caci E, Sondo E, et al. Thiocyanate transport in resting and IL-4-stimulated human bronchial epithelial cells: role of pendrin and anion channels. J Immunol. 2007;178:5144-53.

7. Gakhar L, Bartlett JA, Penterman J, et al. PLUNC is a novel airway surfactant protein with anti-biofilm activity. PLoS One. 2010;5:e9098.

8. Song Y, Salinas D, Nielson DW, et al. Hyperacidity of secreted fluid from submucosal glands in early cystic fibrosis. Am J Physiol Cell Physiol. 2006;290:C741-9.

9. Quinton PM. Role of epithelial HCO3 transport in mucin secretion: lessons from cystic fibrosis. Am J Physiol Cell Physiol. 2010;299:C1222-33.

10. Quinton PM. Cystic fibrosis: impaired bicarbonate secretion and mucoviscidosis. Lancet. 2008;372:415-7.

11. Derichs N, Jin BJ, Song Y, et al. Hyperviscous airway periciliary and mucous liquid layers in cystic fibro- sis measured by confocal fluorescence photobleaching. FASEB J. 2011;25:2325-32.

12. Joo NS, Cho HJ, Khansaheb M, et al. Hyposecretion of fluid from tracheal submucosal glands of CFTRdeficient pigs. J Clin Invest. 2010;120:3161-6.

13. Choi JY, Khansaheb M, Joo NS, et al. Substance P stimulates human airway submucosal gland secretion mainly via a CFTR-dependent process. J Clin Invest. 2009; 119:1189-200.

14. Xu W, Zheng S, Goggans TM, et al. Cystic fibrosis and normal human airway epithelial cell response to influenza a viral infection. J Interferon Cytokine Res. 2006;26:609-27.

15. Zheng S, De BP, Choudhary S, et al. Impaired innate host defense causes susceptibility to respiratory virus infections in cystic fibrosis. Immunity. 2003;18:619-30.

16. Sutanto EN, Kicic A, Foo CJ, et al. Innate inflammatory responses of pediatric cystic fibrosis airway epithelial cells: effects of nonviral and viral stimulation. Am J Respir Cell Molec Biol. 2011;44:761-7.

17. Stoltz DA, Meyerholz DK, Pezzulo AA, et al. Cystic fibrosis pigs develop lung disease and exhibit defective bacterial eradication at birth. Sci Transl Med. 2010;2:29ra31.

18. Sun X, Sui H, Fisher JT, et al. Disease phenotype of a ferret CFTR-knockout model of cystic fibrosis. J Clin Invest. 2010;120:3149-60.

19. Sun X, Yan Z, Yi Y, et al. Adeno-associated virustargeted disruption of the CFTR gene in cloned ferrets. J Clin Invest. 2008;118:1578-83.

20. Rogers CS, Stoltz DA, Meyerholz DK, et al. Disruption of the CFTR gene produces a model of cystic fibrosis in newborn pigs. Science. 2008;321:1837-41.

21. Ostedgaard LS, Meyerholz DK, Chen JH, et al. The $\Delta$ F508 mutation causes CFTR misprocessing and cystic fibrosis-like disease in pigs. Sci Transl Med. 2011;3:74ra24.

22. Meyerholz DK, Stoltz DA, Namati E, et al. Loss of cystic fibrosis transmembrane conductance regulator function produces abnormalities in tracheal development in neonatal pigs and young children. Am J Respir Crit Care Med. 2010;182:1251-61.

\title{
S12.4 \\ THE ROLE OF MUCIN-INTERACTOME IN THE INNATE DEFENSE OF THE LUNG
}

\author{
Mehmet Kesimer, Ph.D. ${ }^{1,2}$, Rui Cao, Ph.D. ${ }^{1,2}$, Tiffany Wang ${ }^{1,2}$ and John K. Sheehan ${ }^{1,2}$ \\ 1. Biochemistry \& Biophysics, UNC at Chapel Hill, Chapel Hill, NC, USA; 2. CF \& Pulmonary Research \& \\ Treatment Ctr., UNC, Chapel Hill, NC, USA
}

Chronic lung diseases such as cystic fibrosis (CF), chronic bronchitis (CB) and asthma are characterized by a hypersecretion of mucus, which often exacerbates morbidity and hastens mortality. A characteristic of these diseases is that they are often accompanied by mucus stasis and enhanced susceptibility to infection and inflamma- tion. The molecular basis for the optimum mucus properties that ensure effective flow remain unexplained.

We have previously shown that there are at least 135 different proteins readily identifiable in respiratory mucus. Our observations revealed that mucus is comprised of an array of biomolecules ranging in molecular 
weight from around $6 \mathrm{kD}$ to $100 \mathrm{MD}$. These may be split into two distinct groups, the first and major group being globular type proteins of MW between $6 \mathrm{kD}$ to $200 \mathrm{kD}$ with a diverse array of proposed functions. The second group is the mucins, which are large space-filling glycoconjugates typically of MW $200 \mathrm{kD}$ to $100 \mathrm{MD}$, most of this mass being carbohydrate in origin. Our studies have yielded evidence that at least 30 of these proteins are involved in distinct, very high molecular weight, multiprotein complexes centered around the gel forming mucins MUC5B and MUC5AC. These complexes constitute a discrete secretory entity we call the "mucin interactome." Based on these studies, we hypothesize that the key rheological functions of mucus do not emanate from the biophysical properties of the mucins themselves but from the dynamic interactions between mucins and globular proteins to form the functioning mucus gel.

To determine the composition of the mucin interactome we have undertaken a combination of biochemical methods such as $\mathrm{S} 1000$ gel chromatography, $\mathrm{CsCl}$ density gradient centrifugation, co-sedimentation and coimmunoprecipitation experiments combined with LCMS/MS mass spectrometry. Proteomics analysis of these steps revealed that the gel forming mucins MUC5B and MUC5AC and membrane tethered mucins MUC1, 4 and 16 are predominant but associated with at least 30 other proteins. The number of the mucin interacting proteins identified was three times lower after using chaotropic agents and/or detergents, indicating that these complexes are affected by such agents. Our rheological experiments indicated an extreme effect of Triton and different dilutions of $\mathrm{GuHCl}$ on the reduction of viscosity and abolishing the network viscoelasticity. Our data revealed a number of protein candidates that resist dissociation from the mucin-interactome of HTBE secretions after such treatments such as LPLUNC1, glutathione S-trans, DMBT1, defensin alpha, surfactant-associated protein B, trefoil factor, complement C3, PLUNC, polymeric-IG receptor, gelsolin, WAP four-disulfide protein2. While the range of specific protein functionalities can be viewed as surprisingly large, e.g. antimicrobial, anti-protease, anti-oxidant and wound healing, we argue that they truly fall into just one general category, i.e., innate host defense. In other words, the mucus gel is part and parcel of innate immunity.

These binding partners of mucins may have a function in modulating mucin/mucus conformation and interactions. On the other hand, just because protein components are attached to the mucins does not necessarily mean that they will modify the rheology in a significant way. In many instances, we might intuit that the mucins carry protective proteins that do not interfere directly in the mechanical properties of the mucus. Therefore, we surmise that the mucin interactome in the airways can be functionally separated into two main roles: 1. Mucin/mucus structure and organization: Interacting proteins may play a role in the mucin structure and organization for effective mucociliary clearance and increased stability of the barrier function. 2 . Enhanced action for innate immune host defense: Highly glycosylated mucins possibly protect the interacting proteins from proteolytic attacks, concentrate them in the region of action, and extend their lifetimes to exert their important biological functions in the respiratory epithelia.

This project is funded by NIH/NHLBI (1R01HL103940).

References: 1 . Kesimer M, Kirkham S, Pickles R, et al. Tracheobronchial air-liquid interface cell culture: a model for innate mucosal defense of the upper airways? Am J Physiol Lung Cell Mol Physiol. 2009;296:L92-L100.

2. Sheehan JK, Kesimer M, Pickles, RJ. Innate immunity and mucus structure and function. In: Innate immunity to pulmonary infection. Wiley, Chichester (Novartis Foundation 279); 2006. p 155-169.

3. Kesimer M, Scull M, Brighton B, et al. Characterization of exosome-like vesicles released from human tracheobronchial ciliated epithelium: a possible role in innate defense. FASEB J. 2009;23:1858-68.

\title{
S13.1 BONE HEALTH: APPROACH TO DIAGNOSIS AND TREATMENT
}

\author{
Andrea Kelly, M.D., MSCE and Jill L. Brodsky, M.D. \\ Pediatrics, Children's Hospital of Philadelphia, Univ. of Pennsylvania, Philadelphia, PA, USA
}

Increased fracture rates in adults with $\mathrm{CF}$ are wellrecognized(1). Vertebral and rib fractures are particularly concerning in $\mathrm{CF}$ as these fractures can compromise pulmonary and nutritional status and impact quality of life. While reported fracture rates in children are not consistently increased(2-3), increased fracture risk in adults may find its roots in childhood. Thus, preserving bone health and identifying individuals with increased fracture risk are important elements in the care of children and adults with CF.
Bone mineral density (BMD), measured by dual energy X-ray absorptiometry (DXA), is an established method of osteoporosis assessment in adults(4) although interpretation of DXA is less well-established in children. Moreover, in CF, the relationship between DXA measures and fracture risk are not established although suspected. In considering DXA, a review of the International Society for Clinical Densitometry Position Statement is worthwhile(5). In children, osteoporosis requires the presence of both low BMD-Z<-2 (low bone mineral 
content/density for chronologic age) and clinically significant fracture (vertebral, lower extremity long bone, or 2 upper extremity long bone fractures), as in premenopausal women and men<age 50y. For the child/adolescent with diseases that threaten skeletal and nutritional health, spine and whole body DXA is recommended and measures of lean body and fat mass should be considered. Assessing bone mineral content (BMC) relative to muscle mass (partly influenced by height) may improve our understanding of bone health(6). Because bone size highly influences DXA, BMC/BMD should be adjusted for height or height age in children with short stature or delayed puberty. How one implements this recommendation, however, remains a challenge.

Both normal and compromised BMC have been reported in the pediatric and adult CF populations(7-11). Bone mineral accrual during puberty appears compromised in CF. Short stature and delayed puberty, including delayed and decreased peak height velocity, are common in CF(12-13), and DXA-BMD may over-estimate bone mineral deficits. CFF guidelines recommend obtaining DXA in adults and in children age $>8 y$ if $<90 \%$ ideal body weight, FEV1<50\% predicted, prolonged/frequent systemic glucocorticoid use, delayed puberty, or history of fracture(14). Studies examining how often to obtain DXA are lacking, but obtaining repeat studies 6-12 months after initiation of a bone related therapy may be appropriate.

Vertebral compression fractures are frequently asymptomatic. In other cases, acute pain can develop and compromise respiratory status. Compression fractures increase the risk of additional compression fractures. Progressive kyphosis can limit vital capacity and compromise abdominal size, leading to decreased appetite. Thus, lateral spine films may be useful for identifying asymptomatic vertebral compression fractures in individuals with decreased BMD-Z.

Because DXA provides an "areal" measure of BMD, other tools directed at measuring bone qualities may be useful in CF. Peripheral quantitative computed tomography provides a three-dimensional measure of bone density and provides data on not only cortical and trabecular bone but bone geometry and strength. Reference data for children are not yet available(15).

Numerous factors threaten bone health in CF. Laboratory evaluation should be directed at identifying potential mediators upon which to intervene: $25 \mathrm{OHD}$, iPTH, phosphorus, zinc, PIVKA, urine calcium/phosphorus/creatinine (hypercalciuria and tubular phosphate reabsorption). To screen for exogenously induced adrenal insufficiency measure AM ACTH and cortisol. In males consider measuring testosterone; females with irregular menses/amenorrhea: iLH, FSH, prolactin. Urine ntelopeptides may help guide therapy choice.

Two categories of therapeutic agents are available for the treatment of osteoporosis: antiresorptive and anabol- ic. Bisphosphonates have primarily been used in CF (1617), but newer agents will also be reviewed.

Bisphosphonates are antiresorptive agents that improve bone mineral density through their affinity for bone tissue where they concentrate at sites of resorption. Bisphosphonates bind directly to bone mineral, are engulfed by osteoclasts, impair osteoclast resorptive function, and ultimately suppress bone turnover markers. Because each agent varies by affinity for bone binding and degree of osteoclast inhibition, no two agents share the same properties. Bisphosphonates are available in oral and intravenous formulations and, depending upon the agent, are prescribed daily, weekly, monthly, quarterly or yearly. Bisphosphonates are not FDA approved for use in children, but a growing body of literature supports their use for pediatric metabolic bone disorders.

Potential side effects may limit their use in CF. In individuals with gastro-esophageal reflux, esophageal erosions have been described with use of oral bisphosphonates. To decrease this risk, oral bisphosphonates are taken with a full glass of water and the patient upright for at least $30 \mathrm{~min}$. Additionally, oral bisphosphonates should be taken on an empty stomach to increase absorption. Hypocalcemia is more commonly described in patients using intravenous formulations of bisphosphonates and may occur up to several days after the completion of the infusion. Prior to using intravenous bisphosphonates, 25OHD and calcium status should be assessed and deficiencies corrected through supplementation. After the first intravenous infusion, patients may experience flu-like symptoms, bone, joint, and muscle pain; non-steroidal anti-inflammatory agents may provide relief. Symptoms tend to decrease with subsequent infusions.

The incidence of osteonecrosis of the jaw, defined as exposed bone in the maxillofacial area with delayed healing for more than eight weeks despite appropriate care(18), is increased in the setting of bisphosphonate therapy (estimated incidence $0.7 / 100,000$ patient treatment years(18). Active malignancy, concurrent treatment with glucocorticoids, extensive and invasive dental work done before treatment, and severe dental and gingival disease increase the risk.

In 2005, a number of case reports describing unusual, low-energy, subtrochanteric femoral fractures in patients taking the oral bisphosphonate alendronate for 5-10 years(19) raised the concern that long-term bisphosphonate therapy may compromise bone integrity. Currently, conclusive evidence directly linking long-term bisphosphonate use to these fractures is lacking and insufficient to warrant discontinuing these drugs in osteoporotic patients(20). However, individuals appear to derive diminished benefit from bisphosphonate use for more than 5 years(21), and ongoing dialogue discussing risks and benefits of continued therapy is important. 
Teriparatide is the only anabolic agent FDAapproved for treatment of postmenopausal women, men with primary or hypogonadal osteoporosis, and osteoporosis associated with sustained systemic glucocorticoid therapy, at high risk for fracture. While chronically elevated levels of PTH lead to osteoclast activation and reduced bone mineral density, intermittent PTH dosing enhances bone modeling via induction of bone lining cells to differentiate into mature osteoblasts and a direct interaction with osteoblasts that leads to decreased cell death. It is administered as a daily subcutaneous injection and is approved for a 2 year treatment period. Potential side effects include transient hypercalcemia, hypercalciuria, and hyperuricemia. In animal studies, concern for osteosarcoma development in individuals with open epiphyses has been raised, and this drug currently has a FDA black box warning against use in children.

Denosumab was FDA approved in 2010 for treatment of postmenopausal women with osteoporosis at high risk for fracture and patients who have failed or are intolerant to other osteoporosis therapy. It is a human monoclonal antibody to RANK ligand and inhibits osteoclast activation. It is administered via subcutaneous injection every 6 months for a period of 3 years. Prior to initiation of denosumab, vitamin $\mathrm{D}$ and calcium repletion are recommended. Side effects include hypocalcemia, skin irritation/infection at injection site, and pancreatitis. Increases in BMD during treatment are lost within 12 months of therapy discontinuation.

Compromised bone health in CF is common, but the best strategies for prevention, screening, and management remain to be defined.
References: 1. Aris R, et al. Ann Intern Med. 1998;128:186-93.

2. Henderson RC, et al. J Pediatr. 1994;125:208-12.

3. Rovner AJ, et al. J Pediatr. 2005;147:327-31.

4. WHO. World Health Organ Tech Rep Ser. 1994;843:1-129.

5. Gordon CM, et al. J Clin Densitom. 2008;11:43-58.

6. Schoenau E. Horm Res. 2006;65(Suppl 2):2-10.

7. King SJ, et al. Eur Respir J. 2005;25:54-61.

8. Buntain HM, et al. J Paediatr Child Health. 2005;41:317-22.

9. Sermet-Gaudelus I, et al. Am J Respir Crit Care Med. 2007;175:951-7.

10. Rossini M, et al. Bone. 2004;35:771-6.

11. Buntain HM, et al. Thorax. 2004;59:149-55.

12. Landon C, et al. Pediatrician. 1987;14:253-60.

13. Landon C, et al. Am J Dis Child. 1984;138:388-91.

14. Aris RM, et al. J Clin Endocrinol Metab. 2005;90:1888-96.

15. Zemel B, et al. J Clin Densitom. 2008;11:59-74.

16. Aris RM, et al. Am J Respir Crit Care Med. 2004;169:77-82.

17. Aris RM, et al. Am J Respir Crit Care Med. 2000;162:941-6.

18. Khosla S, et al. J Bone Miner Res. 2007;22:1479-91.

19. American College of Rheumatology Position Statement on Atypical Femoral Fractures with LongTerm Bisphosphonate Use. 2010.

20. FDA Drug Safety Communication: Ongoing safety review of oral bisphosphonates and atypical subtrochanteric femur fractures. 2010.

21. Black DM, et al. JAMA. 2006;296:2927-38.

\title{
S13.2 \\ BUILDING BONE WITH BETTER NUTRITION: WHO TO TARGET, WHEN AND HOW?
}

\author{
Kristina L. Penniston, Ph.D., R.D., C.D. \\ 1. Urology, Univ. of Wisconsin School of Medicine \& Public Health, Madison, WI, USA; 2. Clinical Nutrition, Univ. \\ of Wisconsin Hospital \& Clinics, Madison, WI, USA
}

As the survival of patients with cystic fibrosis (CF) improves, the incidence of delayed complications, such as bone disease, increases. Although first described decades ago, bone disease in patients with $\mathrm{CF}$ has become more prevalent as patients age. Estimates of osteopenia and osteoporosis in young adults with CF range from $20-40 \%$. Fracture incidence and rates of kyphosis are higher than in the non-CF population and tend to occur at a younger age. The pathophysiology of bone disease in $\mathrm{CF}$ is multifactorial, and nutritional status alone may not correctly predict fracture risk. Low bone mineral density is correlated with severity of lung disease, yet it is not uncommon in adult CF patients with normal lung function. Peak bone mass is often not achieved in patients with $\mathrm{CF}$, and this can be attributed to multiple factors, including nutrition. Moreover, studies show higher rates of bone loss in adults with CF as compared with age-matched controls. These observations combine to place individuals with $\mathrm{CF}$ at high risk for low bone mineral density.

Bone health is important as it is an important contributor to mobility and therefore a necessary component of a healthy and active life. Bone health is an effector of quality of life, with bone disease associated with multiple decrements, including those related to self-esteem, activity levels, and depression. As a growing number of adult 
patients with $\mathrm{CF}$ are considered for lung transplantation, a life-saving treatment for many, severe bone disease is an exclusion factor.

Proficiency in identifying and managing CF-related bone disease and its sequelae is required of today's CF health care team member, including non-dietitians. In addition to vitamin D deficiency, major diet- or nutrientrelated effectors of CF-related bone disease include: (i) malnutrition, general or protein-calorie; (ii) deficiency of micronutrients important for bone, e.g., vitamin K, magnesium, potassium; (iii) negative calcium balance from malabsorption, corticosteroids or both; (iv) caffeine and alcohol; and (v) dysbiosis secondary to malabsorption, chronic antibiotic use, or both. In addition, inflammation secondary to recurrent infection and sarcopenia secondary to malnutrition, both of which are mediated by diet, are linked with bone disease.

Early prevention strategies and interventions to correct risk factors are important, and all members of the $\mathrm{CF}$ health care team should be involved. Targeted food- and supplement-based therapies, in combination with pharmacologic therapy if needed, can successfully alter the natural course of CF-related bone disease.

References: 1. Matel JL, Milla CE. Nutrition in cystic fibrosis. Semin Respir Crit Care Med. 2009;30:579-86.

2. Morton AM. Symposium 6: Young people, artificial nutrition and transitional care. The nutritional challenges of the young adult with cystic fibrosis: Transition. Proc Nutr Soc. 2009;68:430-40.

3. Sermet-Gaudelus I, Bianchi ML, Garabedian M, et al. European cystic fibrosis bone mineralisation guidelines. J Cyst Fibros. 2011;10:S16-S23.

4. Javier R-M, Jacquot J. Bone disease in cystic fibrosis: What's new? Joint Bone Spine. 2011;Jan 12 [Epub ahead of print].

5. Gore AP, Kwon SH, Stenbit AE. A roadmap to the brittle bones of cystic fibrosis. J Osteoporos. 2010 Dec 16;2011:926045.

6. Pappa H, Thayu M, Sylvester F, et al. Skeletal health of children and adolescents with inflammatory bowel disease. J Pediatr Gastroenterol Nutr. 2011;53:11-25.

7. Lacativa PG, Farias ML. Osteoporosis and inflammation. Arg Bras Endocrinol Metabol. 2010;54:123-32.

8. Elborn JS. How can we prevent multisystem complications of cystic fibrosis? Semin Respir Crit Care Med. 2007;28: 303-11.

9. Bruzzese E, Raia V, Gaudiello G, et al. Intestinal inflammation is a frequent feature of cystic fibrosis and is reduced by probiotic administration. Aliment Pharmacol Ther. 2004;20:813-9.

10. Gilman J, Cashman KD. The effect of probiotic bacteria on transepithelial calcium transport and calcium uptake in human intestinal-like Caco-2 cells. Curr Issues Intest Microbiol. 2006;7:1-5.

\title{
S13.3 \\ EXERCISE FOR BONE HEALTH ACROSS THE AGES AND STAGES OF CF
}

\author{
Anne K. Swisher, Ph.D., P.T., C.C.S. \\ Division of Physical Therapy, West Virginia Univ., Morgantown, WV, USA
}

Numerous studies have shown that people with cystic fibrosis (CF) have low bone density compared to their peers. This is true both in childhood bone accrual and adult bone maintenance. This low bone density is associated with many factors, including chronic inflammation, low body weight, low levels of physical activity and CF-related diabetes (CFRD). Lack of healthy bone is concerning, as risk of fragility fractures increase in the $\mathrm{CF}$ population. Fractures can lead to pain and impaired posture, particularly fractures of the vertebral spine. This impaired posture, in turn, can compromise breathing. Fractures and their sequellae can lead to limitations in ability to participate in important life functions and declines in quality of life for these patients.

Exercise and physical activity are important ways to improve and maintain bone health in people with $\mathrm{CF}$ at any age. Exercise is thought to work through mechanical forces of adequate ground reaction force, combined with appro- priate rest intervals, and of increasing stimulus over time in order to cause a positive balance in bone deposition and/or resist resorption. Recent studies regarding chronic inflammation in CF also suggest that the anti-inflammatory effects of regular physical activity and exercise may help fight bone loss through other mechanisms than mechanical forces. Recently, it has been shown that both CFTR and vitamin D receptors are present in skeletal muscle; therefore, muscle contraction may affect, or be affected by these proteins.

Bone-building exercise is typically considered to involve high-velocity impact, which often involves activities such as jumping. In addition, resistance training imparts a strain to bone through tendinous attachment which stimulates a site-specific response. Postural training, particularly of the thoracic spine and ribcage, are especially important in $\mathrm{CF}$, as this is a site of risk for fragility fracture. Aerobic conditioning, typically using weight-bearing activity, can also stimulate bone retention. Recently, peak aerobic capacity was shown to be the 
strongest predictor of bone mineral density in patients with CF. Thus, improving overall exercise capacity may additionally benefit bone density.

However, the art of exercise prescription for bone health comes into play when determining the most appropriate exercise for a specific patient in his or her specific circumstances. This presentation will discuss principles of bone health exercise for patients of different ages (infancy, pre-pubertal, adolescence, adulthood, and aging with $\mathrm{CF}$ ) as well as adapting for severity of lung disease and other situations such as the pregnant patient with $\mathrm{CF}$, or the patient who already has osteoporosis. In the end, the exercise must be the "best" exercise that the patient is willing and likely to perform.

References: 1. Swisher AK. Not just a lung disease: Peripheral muscle abnormalities in cystic fibrosis and the role of exercise to address them. Cardiopulm Phys Ther J. 2006;17:8-12.

2. Sermet-Gauldelus I, Bianchi ML, Garabedian J, et al. European cystic fibrosis bone mineralization guidelines. J Cyst Fibros. 2011;10(Suppl 2):S16-S23.
3. Petersen AMW, Pedersen BK. The anti-inflammatory effect of exercise. J Appl Physiol. 2005;98:1154-62.

4. Dodd JD, Barry SC, Barry RB, et al. Bone mineral density in cystic fibrosis: benefit of exercise capacity.

J Clin Densitom. 2008;11:537-42.

5. Garcia ST, Giraldez-Sanchez MA, Ramos PC, et al. Bone health, daily physical activity and exercise tolerance in cystic fibrosis patients. Chest. 2011; Feb 3 (epub ahead of print).

6. Hind K, Burrows M. Weight-bearing exercise and bone mineral accrual in children and adolescents: A review of controlled trials. Bone. 2007;40:14-27.

7. Hind K, Truscott JG, Conway SP. Exercise during childhood and adolescence: A prophylaxis against cystic fibrosis-related low bone mineral density? J Cyst Fibros. 2008;7:270-6.

8. Orr R. "Exercise and CF-related osteoporosis, diabetes and low fat free mass". Presented at Australasian Cystic Fibrosis Conference. Sydney, Australia, 2007.

\title{
S13.4 \\ THE BARE BONES OF CF BONE HEALTH CARE EDUCATION
}

\author{
Melenie A. Meyers, R.N. \\ Adult CF Center, University of Cincinnati, Cincinnati, $\mathrm{OH}$, USA
}

Cystic fibrosis (CF) affects 30,000 children and adults in the United States (70,000 worldwide). More than $45 \%$ are adults with a median age in the mid 30 s due to a progressive increase in life expectancy over the past three decades.

CF bone disease, i.e., osteopenia, osteoporosis and increased risk of bone fractures, is a common problem occurring in $50-75 \%$ of adult $\mathrm{CF}$ patients that can readily be assessed by measuring bone density with dual energy x-ray absorptiometry (DEXA).

Based on CF Foundation Registry data, osteoporosis occurs in $2.6 \%$ of adults and $0.2 \%$ of children with $\mathrm{CF}$, and osteopenia occurs in $3.3 \%$ of adults and $0.2 \%$ of children with CF. These estimates are likely low since screening DEXA scans are not done routinely in all patients. Thus, while CF bone disease is clearly a problem in adults, it is also important in pediatric CF patients.

The importance of CF bone disease should not be underestimated since it is thought to increase mortality by $5-10 \%$. The clinical consequences of CF bone disease include pain due to cough-induced rib fractures, which can lead to impaired respiratory function from reduced ventilation and cough, reluctance to perform airway clearance and reduced exercise. Others include kyphosis, delayed bone healing, digestive problems, transplant viability, and psychosocial issues.

Education is a cornerstone of preventing CF-related bone disease but requires addressing the myriad risk fac- tors affecting bone health. Education is especially important in pediatric programs since bone health in later life is established at an early age. Puberty is critical for bone development and bone mass peaks by about age 30 and serves as a "bone reservoir" for the remainder of life. Improved comprehension of fracture prevention, monitoring practices, testing algorithms, and features of $\mathrm{CF}$ predisposition to low bone mineral density are all important to education programs.

Learning to fold into our practice, monitoring, screening and education of the multitude of CF complications is a challenge. As health care providers, we have the responsibility of educating ourselves, staff, patients and families about $\mathrm{CF}$ and its many complications.

Education, prevention, early recognition and treatment are the most effective strategies for sustaining bone health to help maintain the quality of life of many individuals with $\mathrm{CF}$.

Utilizing "best practices" methods, screening and educational tools that deliver a consistent message that can be shared across the many CF Centers will help to improve $\mathrm{CF}$ care and outcomes.

References: 1. Cystic Fibrosis Foundation, Patient Registry 2006 Annual Report. Bethesda, MD: Cystic Fibrosis Foundation.

2. Aris RM, Merkel PA, Bachrach LK, et al. Guide to Bone Health and Disease in Cystic Fibrosis. J Clin Endocrinol Metab. 2005;90:1888-96. 


\title{
S14.1 \\ PREDICTION OF CLINICAL DRUG EFFECT ON ION TRANSPORT BY TRANSLATIONAL HUMAN EX VIVO ASSAYS
}

\author{
Nico Derichs, M.D. \\ CFTR Biomarker Centre, Charité University Berlin, Berlin, Germany
}

Defective CFTR-mediated chloride transport is a prominent feature in cystic fibrosis (CF). The CFTR basic defect in native human $\mathrm{CF}$ tissues manifests itself as absent or reduced function of the CFTR chloride channel. Modulation of ion transport is an attractive strategy for treatment of $\mathrm{CF}$. The rationale is that modifying the ion transport properties of CF epithelial cells towards normal would be of clinical benefit. Having good pre-clinical bio-assays to prioritize ion channelmodulating $\mathrm{CF}$ drugs is important in streamlining the $\mathrm{CF}$ drug pipeline in selecting the very best drug candidates to move forward in clinical trials. At present the primary endpoint in pre-clinical evaluation of candidate drugs is electrophysiological responses in well-differentiated primary cultures of human bronchial epithelial cells from CF subjects. There are caveats in the use of this model, however, as e.g. it requires cell growth in culture for at least 3 weeks, where phenotype changes can occur and the model might not be representative of the in vivo condition. Moreover, prediction of clinical drug effect by in vitro data has been shown to be difficult for some CFTR modulating compounds in clinical trials. This leads to the development and optimization of alternative pre-clinical surrogate assays of epithelial cell function in the airways and intestine, two important and accessible CF tissues.

Intestinal current measurement (ICM) is a relatively simple ex vivo test which involves native human rectal biopsies. ICM has been used for genotype-phenotype studies (1-3) and for difficult CF diagnosis (4-7). In recent years, it was further developed for preclinical drug development in CF. Similar studies can be performed with ex vivo native bronchial tissue from human lung explants (8). There are at present no data from clinical trials on changes in ICM induced by disease modifying drugs, however substantial experience has been gained in preclinical human ex vivo corrector studies (9). ICM may be useful in phase IIa and b clinical trials in adult $\mathrm{CF}$ patients, CF children and infants. Preliminary data on intra-individual variability confirm the promising role of ICM as outcome parameter in CF therapeutic studies (Derichs N, unpublished), although more information on repeatability is needed. Another limitation of the ICM is the very low number of centers with expertise. In addition, the short viability of the rectal biopsies impairs transport to a central laboratory for analysis. ICM has at present most application in preclinical drug testing of potentiators and correctors, since the translation of these CFTR modulators from in vitro efficacy to in vivo clini- cal trials can be optimized by ex vivo incubation studies on native human tissues. Under appropriate incubation conditions intestinal biopsies will remain viable and functional for at least $24 \mathrm{~h}$, making the testing of slowacting CFTR correctors feasible (8). In preclinical ICM corrector studies on F508del homozygous CF patients, defective CFTR-mediated chloride secretion could be corrected to levels of $25 \%$ of healthy control after 24 hour ex vivo incubation with corrector-29 (10). However, as is true for NPD, the minimal clinically relevant improvement in ICM is still unknown.

In summary, recent experiences support the utility of ex vivo assays like ICM in native rectal tissue for $\mathrm{CF}$ drug development. Preclinical testing of candidate $\mathrm{CF}$ drugs can help to prioritize and optimize compounds for translation into clinical trials.

References: 1. Veeze HJ, Halley DJ, Bijman J, et al. Determinants of mild clinical symptoms in cystic fibrosis patients. Residual chloride secretion measured in rectal biopsies in relation to the genotype. J Clin Invest. 1994;93:461-6.

2. Bronsveld I, Mekus F, Bijman J, et al. The European CF Twin and Sibling Study Consortium. Residual chloride secretion in intestinal tissue of deltaF508 homozygous twins and siblings with cystic fibrosis. Gastroenterology. 2000;119:32-40.

3. Hirtz S, Gonska T, Seydewitz $\mathrm{HH}$, et al. CFTR $\mathrm{Cl}^{-}$ channel function in native human colon correlates with the genotype and phenotype in cystic fibrosis. Gastroenterology. 2004;127:1085-95.

4. De Jonge HR, Ballmann M, Veeze H, et al. Ex vivo CF diagnosis by intestinal current measurements (ICM) in small aperture, circulating Ussing chambers. J Cyst Fibros. 2004;3(S2):159-63.

5. Derichs N, Sanz J, von Kaenel T, et al. Intestinal current measurement for diagnostic classification of patients with questionable cystic fibrosis: validation and reference data. Thorax. 2010;65:594-9.

6. De Boeck K, Derichs N, Fajac I, et al. ECFS Diagnostic Network Working Group. New clinical diagnostic procedures for cystic fibrosis in Europe. J Cyst Fibros. 2011;10(S2):S53-66.

7. Hug MJ, Derichs N, Bronsveld I, et al. Measurement of ion transport function in rectal biopsies. Methods Mol Biol. 2011;741:87-107.

8. Derichs N, Zander I, Hyde R, et al. Human lung explant tissue as a new ex vivo model for preclinical priorisation of CFTR pharmacotherapy. Pediatr Pulmonol. 2009;4(S32): 103. 
9. Derichs N, Knoll J, Hyde R, et al. Preclinical evaluation of CFTR modulators in ex vivo human rectal tissue. Pediatr Pulmonol. 2009;44(S32):292.
10. Derichs N, Tran D, Namkung W, et al. Correction of F508del-CFTR in human airway epithelia and ex vivo rectal biopsies by s-cis-locked bithiazole corrector-29. (submitted)

\title{
S14.2 MEASURING CFTR ACTIVITY IN CLINICAL TRIALS: IN VIVO ASSAYS
}

\author{
Steven M. Rowe, M.D., MSPH \\ CF Research Center, UAB at Birmingham, Birmingham, AL, USA
}

$\mathrm{CF}$ is caused by mutations in the gene encoding the $\mathrm{CF}$ transmembrane conductance regulator (CFTR), an epithelial anion channel involved in chloride and bicarbonate transport. Absent or defective CFTR results in abnormal ion transport in the airways, sinuses, sweat duct, gastrointestinal tract, hepatobiliary tract, and vas deferens. In the lung, mucus stasis ensues, resulting in chronic infection and end organ dysfunction, and is a common cause of morbidity and early mortality. Although there are over 1500 disease-causing mutations in CFTR, they can be categorized into several major classes based on the molecular pathogenesis induced by each mutation (1). CFTR mutations can also be generally grouped by severity of the anion transport abnormality conferred by the mutation. While numerous factors alter the genotype-phenotype correlation in $\mathrm{CF}$, including environmental factors and genetic modifiers to CFTR activity, the severity of the ion transport defect is also correlated with long-term outcome (2). As such, measurement of CFTR activity in CF subjects can confer diagnostic and prognostic information, suggesting the importance of accurately measuring CFTR activity. As restoring CFTR function through small molecule pharmaceuticals is one therapeutic approach that has shown considerable promise for the treatment of CF (3), accurate and reproducible measurements of CFTR activity is also essential to evaluating the efficacy of these CFTR modulators in development.

The traditional measurement of CFTR activity involves measurement of sweat chloride concentration following stimulation of sweating with the cholinergic agonist pilocarpine, and has long been used as a diagnostic marker in CF. Standardization of the method using the Macroduct collection system has facilitated use of sweat chloride analysis in the context of clinical trials, and also allows analysis in a central laboratory blinded to treatment assignment (4). Use of sweat chloride analysis for the evaluation of the efficacy of the CFTR potentiator VX-770 and the CFTR F508del processing corrector VX-809 has been highly informative. As predicted by genotype-phenotype correlations in $\mathrm{CF}$, changes in sweat chloride following modulation of CFTR have been highly sensitive to small changes in CFTR activity, and have allowed relative efficacy to be determined with relatively simple study designs and small numbers of study subjects (3). Emerging methods of sweat testing, including sweat rate analysis by evaporimetry or quantitation of the sweat output from individual glands, can also be used to quantify the function of sweat glands (as opposed to the sweat duct), and may provide additive diagnostic information regarding the impact of CFTR modulation on glandular epithelium. Whether this method confers additional information in the context of CFTR modulation remains to be determined.

Unlike sweat chloride analysis, nasal potential difference (NPD) measurements allow the isolation of sodium and chloride transport, providing a means to independently analyze CFTR activity, $\mathrm{ENaC}$ function, and ion transport induced by alternative chloride channels. The technique is performed in vivo, and can also be used to evaluate proof-of-concept studies involving local (e.g., nasal) administration of biologic agents, including pharmaceuticals and gene therapy vectors. NPD has been sensitive to the detection of improved chloride transport induced by various CFTR modulators, including premature termination codon suppressors, CFTR potentiators, and gene therapy agents, and also has been sensitive to agents that inhibit ENaC activity (4). Although the measurement requires specialized equipment and training to conduct appropriately, recent advances in methodology have improved the quality of tracings, provided a means for central interpretation and analysis, and greatly facilitated ease of use, providing a means to use NPD in clinical trials involving a large number of operators and participants (5). The technique can also be adapted to measure CFTR activity in the lung, however potential difference measurements do require a greater number of subjects to demonstrate a detectable difference in CFTR activity compared to sweat chloride analysis, particularly when evaluating an agent with marginal activity. Similar to NPD, intestinal current measurements (ICM) provide a means to isolate sodium and chloride transport. An added utility of the measure is that it provides a means to test the activity of ion transport modulators ex vivo on full-thickness human tissues. Recent standardization of ICM measurement technique may provide a means to use the method in multicenter trials.

As data regarding the modulation of CFTR activity involving a number of different treatment strategies continue to emerge from long-term trials that also measure clinical outcome, use of CFTR activity measures may become useful as a surrogate outcome measure in $\mathrm{CF}$. 
Such an advance may further facilitate the clinical evaluation of CFTR modulators in individuals with rare CFTR mutations or atypical (e.g., nonclassic) manifestations.

References: 1. Rowe SM, Miller S, Sorscher EJ. Cystic fibrosis. N Engl J Med. 2005;352:1992-2001.

2. Wilschanski M, Dupuis A, Ellis L, et al. Mutations in the cystic fibrosis transmembrane regulator gene and in vivo transepithelial potentials. Am J Respir Crit Care Med. 2006;174:787-94.

3. Accurso FJ, Rowe SM, Clancy JP, et al. Effect of $\mathrm{VX}-770$ in persons with cystic fibrosis and the
G551D-CFTR mutation. N Engl J Med. 2010;363:1991-2003.

4. Rowe SM, Accurso F, Clancy JP. Detection of cystic fibrosis transmembrane conductance regulator activity in early-phase clinical trials. Proc Am Thorac Soc. 2007;4:387-98.

5. Solomon GM, Konstan MW, Wilschanski M, et al. An international randomized multicenter comparison of nasal potential difference techniques. Chest. 2010;138:919-28.

\title{
S14.3 \\ PULMONARY BIOMARKERS OF INFLAMMATION IN CF LUNG DISEASE
}

\author{
Scott D. Sagel, M.D. \\ Pediatrics, Children's Hospital Colorado \& Univ. of Colorado School of Medicine, Aurora, CO, USA
}

Cystic fibrosis (CF) lung disease is characterized by a self-perpetuating cycle of airway obstruction, chronic bacterial infection, and vigorous inflammation that results in bronchiectasis, progressive obstructive lung disease, and marked shortening of life expectancy. Since airway inflammation plays a central role in the progression of CF lung disease, inflammatory biomarkers that can be used to monitor disease activity and evaluate response to therapy would be extremely valuable. Many inflammatory cells, mediators, and enzymes are involved in the complex pathophysiology of CF lung disease, so that there are many possible biomarkers to study and there is a high degree of redundancy $(1,2)$. Informative biomarkers may be identified through discovery-based proteomic or metabolomic approaches or through targeted measurement of purported candidates (cytokines, proteases, oxidants, matrix components, tissue breakdown products).

The ideal biomarker would be clinically and biologically relevant, reproducible, sensitive and specific to treatment effects, and feasible (3). At present, no single inflammatory biomarker clearly meets all of these criteria. Individual biomarkers will be appropriate in trials of targeted anti-inflammatory therapeutic agents such as a specific anti-cytokine or anti-protease drugs. However, for clinical trials of anti-inflammatory treatments that target multiple pathways of the inflammatory response or for therapeutic agents aimed at correcting cystic fibrosis transmembrane conductance regulator (CFTR) protein function or restoring airway surface liquid, a combination of biomarkers will likely prove more valuable. This symposium presentation will review the current state of knowledge of biomarkers of inflammation relevant to $\mathrm{CF}$ lung disease, and the tools to measure inflammation. This presentation will critically examine the existing data supporting the validity of pulmonary biomarkers in $\mathrm{CF}$, with an eye towards their application as surrogate endpoints or outcome measures in CF clinical trials.
Due to the localized nature of enhanced inflammation in $\mathrm{CF}$, respiratory secretions from the lower airway are the optimal source of inflammatory cells and soluble mediators, and can be obtained directly or indirectly from the airways. The most common direct method is by performing bronchoscopy with bronchoalveolar lavage (BAL) or bronchial washing. Much of what has been learned about infection and inflammation in the CF lung has been through the use of BAL and much of the experience evaluating the "biologic efficacy" of therapeutics in $\mathrm{CF}$ has been through the use of BAL (reviewed in reference (2)). BAL offers the advantages of being able to be performed in any age group, and for its ability to sample the same area of the lung for studies that require repeat sampling (as is commonly done with intervention studies). Disadvantages include the need for an experienced team to perform the procedure, the need for sedation to perform the procedure, the inability to conveniently perform repeat sampling more than once or twice throughout an intervention study, and the high costs associated with performing the procedure. As a result of these drawbacks, investigators have relied on spontaneously expectorated sputum for many years, but this requires patients to have enough lung involvement and resultant respiratory secretions that allow regular sputum production. Thus, it is not very useful for examining inflammation in early lung disease, and in young children regardless of disease severity, since young children have difficulty expectorating sputum. To overcome the obstacle of obtaining sputum from subjects with milder lung disease, inducing a sputum specimen with hypertonic saline has been used to obtain lower airway secretions, and much effort has been expended in recent years in an attempt to validate the methodology of obtaining, processing, and assaying induced sputum (reviewed in detail in reference (2)). However, sputum induction is difficult to perform in patients age 8 years and younger, and multicenter studies 
have largely limited inclusion to those 10 years of age or greater. Additionally, biomarkers of inflammation in both induced and expectorated sputum show considerable variability, especially in comparison to BAL.

Other ways of indirectly assessing markers of inflammation in the lower airways have been considered, including exhaled gases and breath condensate, but they too have their limitations. Exhaled NO (eNO) is reduced in CF (4); therefore, consideration could be given to measuring eNO in exhaled breath in a study of an anti-inflammatory drug that might impact the pathway leading to the upregulation of NO. While investigators have measured some inflammatory mediators in exhaled breath condensate, the usefulness of these measures as outcomes remains to be defined as well.

A systemic marker of lung inflammation would be ideal. Blood and urine can be obtained from subjects of any age and disease severity, and may reflect the status of inflammation throughout the lung, rather than one segment as is assessed by BAL, or heterogeneous segments as is assessed with sputum. However, a systemic marker may not be sensitive enough to detect a meaningful change in lung disease, given that the inflammatory response to infection in the CF lung is largely confined to the lung.

In $\mathrm{CF}$, we need more information about how pulmonary biomarkers relate to lung disease development, severity, and progression, and how they change in response to treatments. In addition, there is a relative paucity of data of how pulmonary biomarkers may be affected by concurrent therapies, or how they relate to important clinical outcomes in $\mathrm{CF}$, including rate of decline in FEV1, pulmonary exacerbation frequency, and mortality. In the future, pulmonary biomarkers will likely be useful in predicting disease progression, indicating the onset and resolution of a pulmonary exacerbation, and assessing response to current therapies or candidate therapeutics.

Supported by the CF Foundation (SAGEL07A0) and the National Institutes of Health (U54 HL096458-07).

References: 1. Chmiel JF, Berger M, Konstan MW. The role of inflammation in the pathophysiology of $\mathrm{CF}$ lung disease. Clin Rev Allergy Immunol. 2002;23:5-27.

2. Sagel SD, Chmiel JF, Konstan MW. Sputum biomarkers of inflammation in cystic fibrosis lung disease. Proc Am Thorac Soc. 2007;4:406-17.

3. Mayer-Hamblett N, Ramsey BW, Kronmal RA. Advancing outcome measures for the new era of drug development in cystic fibrosis. Proc Am Thorac Soc. 2007;4:370-7.

4. Balfour-Lynn IM, Laverty A, Dinwiddie R. Reduced upper airway nitric oxide in cystic fibrosis. Arch Dis Child. 1996;75:319-22.

\title{
S14.4 \\ NEW INSIGHTS INTO LUNG STRUCTURE, FUNCTION AND MUCOCILIARY CLEARANCE
}

\author{
Christiane De Boeck, M.D., Ph.D. \\ University Hospital Gasthuisberg, Leuven, Belgium
}

In $\mathrm{CF}$ lung disease, the course of FEV1 relates to long-term outcome. However, using FEV1 as the primary outcome parameter in phase 3 trials has become difficult, since the mean year to year change in FEV1 is low. Apart from adolescence, during which it is higher, the year to year change is only in the order of $1 \%$ (1). Even though the mean change is low, the loss of lung function is not homogeneously distributed between patients: 15$20 \%$ of children experience a year to year change of $10 \%$ or more and $30-40 \%$ have a year to year change $>5 \%$. One could focus on subjects at higher risk of loss of lung function but then the study would lose in terms of generalizability: the findings may not be applicable to subjects with a more stable disease course. When a large treatment effect is anticipated, the use of FEV1 as primary outcome remains a good option e.g., such as proven in the evaluation of VX-770.

The use of pulmonary exacerbations as outcome in $\mathrm{CF}$ clinical trials is also not obvious. We do not have a universal definition for this item. Pulmonary exacerbations requiring treatment with intravenous antibiotics are a major driver of lung disease progression, but they are infrequent in young and/or rather healthy subjects with CF (2). Less stringent definitions of pulmonary exacerbations have been used or are under evaluation but it is less certain how these relate to long-term loss of lung function.

The need for new surrogate outcome parameters is thus obvious: the multiple breath inert gas washout test (MBW) and chest CT are prime candidates, especially in children or subjects with mild lung disease.

$\mathrm{CF}$ lung disease starts in the small airways and FEV1 is insensitive to changes in the small airways. MBW is non-invasive and provides information about this "silent" lung zone where disease starts $(3,4)$. The result of MBW, a test of gas mixing efficiency, is usually reported as the lung clearance index (LCI) i.e., the number of "lung turnovers" (FRCs) required to clear the lungs of an inert gas to 1/40th of the starting concentration. LCI is more sensitive than FEV1 (4). LCI has been shown to be responsive to interventions such as treatment with intravenous antibiotics (5), hypertonic saline (6) and rhDNAse (7). Recent data link early LCI abnormalities to longer term lung disease course: an abnormal LCI at preschool age predicted an abnormal FEV1 at school age 
(8). Several issues remain to be resolved: which MBW apparatus is best, which inert gas is most appropriate, are results obtained by different techniques comparable, what is the test repeatability, what constitutes a significant change. Preliminary data are promising: given the increased sensitivity of LCI and depending on the research question, power calculations come up with numbers around 50 children in each study arm (6). This compares favorably to using FEV1 as outcome measure, which will require hundreds of children in each treatment arm (9).

The second outcome measure with the potential to solve the current problem of loss of sensitivity is scoring of chest CT. Several studies have proven this parameter's sensitivity. At a mean age of 4 months, 84\% of infants with $\mathrm{CF}$ detected after newborn screening are asymptomatic but $81 \%$ have an abnormal CT (10). At the age of 5 years, 7 of 11 subjects already have bronchiectasis (11). Bronchiectasis scoring on chest CT correlates well with measurement of LCI $(3,12)$. Chest CT has the disadvantage of a radiation burden but it has the advantage of objectively quantitating structural CF lung disease (13). Again, using this parameter as an outcome will offer the advantage of feasibility in terms of study size. Depending on the hypothesis put forward, the sample size calculations for use of chest CT scoring in longterm studies result in about 50 subjects per group (13).

CFTR dysfunction impedes chloride transport to the airway lumen. Together with an increased sodium reabsorption, caused by the loss of CFTR inhibition on ENaC function, this leads to dehydration of the airway surface lining (ASL) fluid. It follows that the mucociliary clearance is greatly reduced, because the cilia are unable to function properly. This theory is challenged. Measurement of the ASL depth and the ASL and mucociliary layer viscosity is possible with phase contrast X-ray imaging (14) or confocal fluorescence photobleaching (15). Also measuring airway liquid absorption and mucociliary clearance by nuclear medicine techniques are interesting biomarkers close to the fundamental defect of CF lung disease (16). These measurements of mucociliary composition and clearance are however still in the exploratory phase and not yet appropriate for Phase 3 clinical trials.

Last but not least, we are eventually entering the era of disease modifying drugs. These treatments with CFTR potentiators and correctors and nonsense mutation readthrough probably have the highest potential in young children with relatively healthy lungs. Paradoxically showing improvement in this population is extremely difficult. Several gating mutations reported are very rare and cannot be studied individually. Therefore we should dare to think out of the box. Providing proof of efficacy in a Phase 3 trial in a small population is impossible. When studying CFTR correctors and potentiators in (pre)school children with a very slow disease progression or in a very limited population, it is appropriate to use a biomarker closely linked to the causal pathway of the disease as primary outcome. CFTR bio-assays such as sweat chloride and nasal potential difference measurement or intestinal current measurements are good candidates (17). An alternative is to only collect safety data especially if efficacy has already been proven in another age category. Proof of clinical benefit can then follow during pharmacovigilance via Phase 4 trials.

References: 1. Liou TG, Elkin EP, Pasta DJ, et al. Year-to-year changes in lung function in individuals with cystic fibrosis. J Cyst Fibros. 2010;9:250-6.

2. Goss CH, Burns JL. Exacerbations in cystic fibrosis. 1: Epidemiology and pathogenesis. Thorax. 2007;62:360-7.

3. Gustafsson PM, De Jong PA, Tiddens HA, et al. Multiple-breath inert gas washout and spirometry versus structural lung disease in cystic fibrosis. Thorax. 2008;63:129-34.

4. Aurora P, Bush A, Gustafsson P, et al. Multiplebreath washout as a marker of lung disease in preschool children with cystic fibrosis. Am J Respir Crit Care Med. 2005; 171:249-56.

5. Robinson PD, Cooper P, Van Asperen P, et al. Using index of ventilation to assess response to treatment for acute pulmonary exacerbation in children with cystic fibrosis. Pediatr Pulmonol. 2009;44:733-42.

6. Amin R, Subbarao P, Jabar A, et al. Hypertonic saline improves the LCI in paediatric patients with $\mathrm{CF}$ with normal lung function. Thorax. 2010;65:379-83.

7. Amin R, Subbarao P, Lou W, et al. The effect of dornase alfa on ventilation inhomogeneity in patients with cystic fibrosis. Eur Respir J. 2011;37:806-12.

8. Aurora P, Stanojevic S, Wade A, et al. Lung clearance index at 4 years predicts subsequent lung function in children with cystic fibrosis. Am J Respir Crit Care Med. 2011;183:752-8.

9. De Boeck K, Vermeulen F, Wanyama S, et al. Inhaled corticosteroids and lower lung function decline in young children with CF. Eur Respir J. 2011;37:1091-5.

10. Sly PD, Brennan S, Gangell C, et al. Lung disease at diagnosis in infants with $\mathrm{CF}$ detected by newborn screening. Am J Respir Crit Care Med. 2009;180:146-52.

11. Stick SM, Brennan S, Murray C, et al. Bronchiectasis in infants \& preschool children diagnosed with $\mathrm{CF}$ after newborn screening. J Pediatr. 2009;155:623-8. e1.

12. Owens CM, Aurora P, Stanojevic S, et al. Lung Clearance Index and HRCT are complementary markers of lung abnormalities in young children with CF. Thorax. 2011;66:481-8.

13. Sly PD, Ware RS, de Klerk N, et al. Randomised controlled trials in cystic fibrosis: what, when and how? Eur Respir J. 2011;37:991-3.

14. Siu KK, Morgan KS, Paganin DM, et al. Phase contrast X-ray imaging for the non-invasive detection of airway surfaces and lumen characteristics in mouse models of airway disease. Eur J Radiol. 2008;68(3 Suppl):S22-6. 
15. Derichs N, Jin BJ, Song Y, et al. Hyperviscous airway periciliary and mucous liquid layers in CF measured by confocal fluorescence photobleaching. FASEB J. 2011;25:2325-32.

16. Corcoran TE, Thomas KM, Myerburg MM, et al. Absorptive clearance of DTPA as an aerosol-based bio- marker in the cystic fibrosis airway. Eur Respir J. 2010;35:781-6.

17. De Boeck K, Derichs N, Fajac I, et al. New clinical diagnostic procedures for cystic fibrosis in Europe. $\mathrm{J}$ Cyst Fibros. 2011;10(Suppl 2):S53-66.

\title{
S15.1 LESSONS LEARNED FROM THE DENUFOSOL TRIALS
}

\author{
Richard B. Moss, M.D. \\ Pediatrics, Stanford University, Palo Alto, CA, USA
}

On December 17, 2010 the results of the first Phase 3 trial of denufosol (TIGER-1, "Transport of Ions to Generate Epithelial Rehydration") were published (1). The results of this 24 week, 352 patient double-blind placebo-controlled protocol in CF patients of any genotype $\geq 5$ years old with FEV1 $\geq 75 \%$ predicted were positive, in that the pre-specified primary FEV1 endpoint was met, and a 24 week open-label extension seemed to confirm this lung function effect, suggesting, in the authors' heady words, "denufosol has efficacy and safety profiles suitable for early intervention in cystic fibrosis lung disease." The developers of denufosol, Inspire Pharmaceutials, and the CF community looked forward to confirmation in the FDA-required second Phase 3 trial (TIGER-2) followed swiftly by submission of a New Drug Application to the FDA for approval. Instead, less than 3 weeks after publication of the TIGER-1 paper, Inspire announced that the 466 patient, 48 week placebo-controlled TIGER-2 trial did not even come close to meeting any of its endpoints (2). Inspire stock tanked; the company lost $\$ 400 \mathrm{~m}$ in one day (3). It promptly shelved the program, restructured and laid off employees; by mid-May its remaining ophthamologic franchise was acquired by Merck (4). Inspire expired. What happened?

Denufosol is a third-generation nucleotide P2Y2 agonist derived from UTP with increased stability on the respiratory epithelial mucosal surface $\left(\mathrm{t}_{1 / 2} \sim 3 \mathrm{hr}\right)$. Much elegant in vitro work has established that denufosol increases rates of PIP2 hydrolysis, increasing IP3 and release of $\mathrm{Ca}^{2+}$ intracellularly; this increases chloride secretion through calcium-activated chloride channels (CACC), stimulates ciliary beat frequency, increases mucin secretion from goblet cells and surfactant from type II alveolar cells, and inhibits sodium absorption via the epithelial sodium channel, resulting in improved airway hydration and increased mucociliary transport $(5,6)$. Because it is hydrolyzed by endogenous nucleotidases at the epithelial surface and rapidly metabolized after IV infusion there is virtually no systemic exposure and thus little chance for systemic toxicity, as verified in preclinical animal toxicity studies and later in the clinical trials. Denuofosol was formu- lated to be delivered by conventional jet nebulization for clinical trials.

Granting retrospective advantage, it does appear hints of impending disaster can be found in the development program results, but were ignored or misinterpreted by both a commercial enterprise and diverse stakeholder community eager for success for both unique and common reasons. By focusing on these at best ambiguous if not frankly negative harbingers, it is possible that we may learn not to repeat the mistakes of the past.

1. Mechanistic Rationale. The results of TIGER-2 suggest that the preclinical assessment of denufosol's effect on CF respiratory epithelium, clear in so many excellent in vitro experiments, may be flawed. The key finding by researchers at UNC, after the clinical program was well under way, that rate of change-dependent shear stress releases endogenous luminal ATP, thereby activating the CACC to secrete chloride, opens the possibility that this channel may be more physiologically active in CF than previously anticipated, and thus less clinically augmented by pharmacologic stimulation (7). Might exercise, airway clearance regimes or even activities of daily living already be doing the CFTR-bypass work it was supposed denufosol might be needed to accomplish? Now that the molecular identity of at least one CACC has been discovered as TMEM16A it may be possible to evaluate this with more precision (8). The clinical effect of inhaled osmotic agents such as hypertonic saline and mannitol points to the viability of strategies to improve airway surface hydration. But the fate of other investigational drugs activating CACC and/or inhibiting ENaC may be affected by further research in this area.

2. Phase 1. The determination of optimal dose and interval are crucial in clinical drug development. The first trials of denufosol showed single-dose tolerability at up to $200 \mathrm{mg}$ in non-CF adult nonsmokers. In adult and pediatric CF patients Phase 1 highest tested dose was 60 $\mathrm{mg}$ as a single dose or in b.i.d. dosing for 5 days, and since this was well-tolerated this dose was carried forward (9). Although there was somewhat more wheezing at $60 \mathrm{mg}$ vs lower doses, could higher doses have been more effective with acceptable levels of intolerability? If 
acute increases in airway hydration were feared as potential cause of adverse events, strategies to deal with this via dose titration could have been tried. The only pharmacodynamic measure was change in expectorated sputum weight, but this is a fickle outcome that did not show clear dose-response, was only seen after single and not repeat doses, and seems an inappropriate biomarker given the decision to treat only patients with FEV1 $\geq 75 \%$ predicted in Phase 3, as many patients with mild disease do not expectorate.

3. Phase 2. The Phase 2 program for denufosol incorporated several crucial junctures in regime and target population. In the first protocol 89 patients $\geq 8$ years old with FEV $1 \geq 75 \%$ were enrolled in a 28 day controlled trial in randomized parallel groups receiving 20, 40, 60 mg or placebo t.i.d. (10). Patients taking inhaled antibiotics, hypertonic saline, or macrolides were excluded. Tolerability was good. With respect to efficacy, there was no dose-response in FEV1 change from baseline in actively treated patients. While the denufosol-treated pooled group change was significantly better than placebo, the effect in protocol completers was less than intentto-treat, and most importantly, the placebo group $(n=21)$ experienced an unusually sharp decline in FEV1 (adjusted mean decline of $140 \mathrm{~mL}$ or $\sim 2.5 \%$ over 28 days) and other lung function measures, changes ascribed to regression to the mean. In addition there were no differences in CFQ-R, HRCT scores, sputum weight, or exacerbations. Was the calculation that nominal doses $\geq 20 \mathrm{mg}$ achieve initial airway surface concentrations exceeding maximally effective doses as calculated from nasal potential difference measurements, and thus predictive of no doseresponse over the trial dose range, correct (moreover, even if true, is initial concentration enough to count on)? Taken together, the results now seem suspect in indicating any efficacy, given the aberrant placebo decline in lung function, the questionable approach to pharmacodynamic dose-response, and the lack of concurrent changes in multiple clinically relevant outcome measures. Notably, the second Phase 2 trial enrolling 72 patients with FEV1 modified to $60-90 \%$ showed no efficacy and a smaller treatment effect in those with $\geq 75 \%$ FEV1 due to a more typical flat placebo response (11).

4. Phase 3. Based on Phase 2, TIGER-1 extended the approach of selecting patients with FEV $1 \geq 75 \%$ predicted, necessarily reducing magnitude of potential lung function treatment effect. TIGER-1 barely met its primary endpoint of a statistically significant treatment effect on FEV1 (L) at 24 weeks $(45 \mathrm{~mL}, \mathrm{p}=0.047)$. But ominous signs were many: the magnitude was small; the effect of growth on lung volume was not incorporated (nonsignificant trends were seen for FEV1 \% predicted); no secondary endpoints (other spirometric measures, exacerbations, CFQ-R) were attained. The kinetics of response were puzzling for an active drug: both active and placebo FEV1 were down at 28 days and then rose slowly over time. On the other hand, post-hoc analyses of patients on minimal background therapy (0-2 drugs), of adolescents, and of FEV1 loss in patients with exacerbation appeared to suggest efficacy by showing trends to greater denufosol treatment effect than seen in the overall study population $(1,12,13)$. Based on TIGER-1 top line data, TIGER-2 was redesigned after launch to optimize chances for success by extending the placebo-controlled treatment period to 48 weeks, increasing sample size to boost power, and capping entry FEV1 at $110 \%$. Despite all this, TIGER-2 results showed superimposable results for denufosol and placebo in all outcomes.

This sobering story shows that drugs likely to succeed for $\mathrm{CF}$ should undergo continued basic research on mechanism given our substantial ignorance of CF pathogenesis and pathophysiology; that we should confirm optimal dose (preferably by dose-dependent relevant clinical pharmacodynamics), interval and delivery, as well as safety, in Phase 1; that we should obtain robust exploratory efficacy endpoints on several congruent biomarker and/or clinical outcomes, with expected placebo responses as well as safety, in Phase 2; and that we should achieve multiple and clinically meaningful as well as statistically significant efficacy outcomes and safety in Phase 3. Short of this, the stakes are too high and the price too dear.

References: 1. Accurso FJ, et al. Am J Resp Crit Care Med. 2011;183:627-34. Epub 2010 Dec 17.

2. Press release, Inspire Pharmaceuticals, Jan 3, 2011.

3. http://seekingalpha.com/article/245167-inspirepharmaceuticals-looking-for-inspiration-following-denufosol failure. Accessed June 22, 2011.

4. http://www.merck.com/newsroom/news-releasearchive/corporate/2011_0516.html. Accessed June 22, 2011.

5. Kellerman D, et al. Pulm Pharmacol Ther. 2008;21:600-7.

6. Clunes MT, et al. Curr Opin Pharmacol. 2008;8:292-9.

7. Button B, et al. Respir Physiol Neurobiol. 2008;163:189-201.

8. Galietta LJ. Biophys J. 2009;97:3047-53.

9. Deterding R, et al. Pediatr Pulmonol. 2005;39:339-48.

10. Deterding R, et al. Am J Respir Crit Care Med. 2007; 176:362-9.

11. Smiley L, et al. J Cystic Fibros. 2006;5:S11.

12. Moss RB, et al. J Cystic Fibros. 2010;9:S20.

13. Accurso FJ, et al. J Cystic Fibros. 2010;9:S21. 


\title{
S15.2 \\ KEY ADVANCES ALONG THE CF PIPELINE
}

\author{
J. Stuart Elborn, M.D., FRCP \\ Centre for Infection \& Immunity \& Adult CF Ctr., Queen's University, Belfast, United Kingdom
}

In the past five years a significant number of new therapies have been developed for the treatment of cystic fibrosis. A number of new inhaled antibiotics are available or are in Phase III clinical trials that will significantly help in controlling chronic infection and so hopefully maintaining lung function (1). How these therapies will be used together remains to be determined. Antiinflammatory therapies, such as those directed against human neutrophil elastase show some promise but are still in early phase clinical trials (2). More novel approaches aimed at regulating downstream inflammatory pathways in immune or epithelial cells and other aspects of innate immunity are currently being investigated but are also only in early phase studies (3).

Advances in therapies for airways clearance also show considerable promise with inhaled mannitol (Bronchitol) in the final stages of clinical development. These studies indicate that Bronchitol significantly improves FEV1 which is sustained over 12 months and also reduces pulmonary exacerbations (4). Bronchitol is likely to be a useful adjunct to current therapies as it is equally effective in those on Pulmozyme as those not.

The most exciting progress in pipeline therapies is in the development of therapies which affect the basic defect of cystic fibrosis. These drugs include PTC124, VX-770 for G551D patients and VX-809 in combination with VX-770 for F508del mutations, and the Gene Therapy programme using a plasmid liposome construct.

PTC124 is designed to overcome stop mutations by causing readthrough in the translational step and so resulting in the manufacture of full length protein. Proof of concept studies have been undertaken and two of these have shown significant changes in nasal potential difference and some improvement in FEV1 $(5,6)$. These results are encouraging and the pivotal phase 3 study has been completed and the results should be available in the very near future.

VX-770 is a small molecule which potentiates CFTR which is expressed on the cell surface (Class III mutations). This means it is likely to be useful across all Class III mutations but it has been particularly studied in patients with the most common, archetypal class III mutation, G551D. In a proof of concept study, VX-770 significantly reduced sweat chloride concentration and nasal potential difference with improvements in FEV1 (7). A pivotal Phase 3 study has been completed and demonstrates significant and sustained improvements in FEV1 by $10 \%$. This is associated with significant improvements in CFQ-R respiratory domain, a significant reduction in pulmonary exacerbations, a significant weight gain and a reduction in sweat chloride concentrations. This demonstrates for the first time that a treatment that corrects the functional, basic defect in vitro translates through to significant improvement in relevant clinical measurements of lung function, symptoms and systemic measurements of CFTR function. VX-770 has also been studied in F508del homozygous patients and shows no significant clinical efficacy though there is a small improvement in sweat chloride concentrations. The most recent study examining the effect of the combination of VX-770 and VX-809 in F508del homozygous patients suggests both drugs have no major safety issues when used together and has demonstrated a modest, potentially meaningful reduction in sweat chloride concentrations (8).

Gene therapy has the potential to overcome the functional defect in CF in all classes of mutations. The UK Gene Therapy consortium has recently completed a single dose, safety study combining an innovative plasmid that expresses CFTR for around 4 weeks and the liposome GL67. This will enter a multidose one year study in 2012. This will determine if this approach has therapeutic potential.

Current pipeline therapies for CF show considerable promise. The improvements in patients with at least one G551D mutation following VX-770 show that CFTR is a tractable target and improving CFTR function results in significant improvements in clinical status. Further results from the current, ambitious programmes of development of therapies which are aimed at treatment of the functional defect in $\mathrm{CF}$ are eagerly awaited.

References: 1. Dudley MN, Loutit J, Griffith DC. Aerosol antibiotics: considerations in pharmacological and clinical evaluation. Curr Opin Biotechnol. 2008; 19:637-43.

2. Brand P, Schulte M, Wencker M, et al. Lung deposition of inhaled alpha(1)-proteinase inhibitor in cystic fibrosis and alpha(1)-antitrypsin deficiency. Eur Respir J. 2009;34:354-60.

3. Banner KH, De Jonge H, Elborn JS, et al. Highlights of a workshop to discuss targeting inflammation in cystic fibrosis. J Cyst Fibros. 2009;8:1-8.

4. Bilton D, Robinson P, Cooper P, et al. Inhaled dry powder mannitol in cystic fibrosis: an efficacy and safety study. Eur Respir J. 2011 Apr 8. [Epub ahead of print]

5. Kerem E, Hirawat S, Armoni S, et al. Effectiveness of PTC124 treatment of cystic fibrosis caused by nonsense mutations: a prospective phase II trial. Lancet. 2008;372:719-27.

6. Sermet-Gaudelus I, De Boeck K, Casimir GJ, et al. Ataluren (PTC124) induces cystic fibrosis transmembrane conductance regulator protein expression and activity in children with nonsense mMutation cystic fibrosis. Am J Respir Crit Care Med. 2010;182:1262-72. 
7. Accurso FJ, Rowe SM, Clancy JP, et al. Effect of VX-770 in persons with cystic fibrosis and the G551DCFTR mutation. New Eng J Med. 2010;363:1991-2003.
8. http://www.vrtx.com/

\title{
S15.3 \\ COMBINATION OF CORRECTORS AND POTENTIATORS FOR F508DEL CFTR
}

\author{
Steven M. Rowe, M.D., MSPH ${ }^{1}$ and Michael P. Boyle, M.D. ${ }^{2}$ \\ 1. UAB at Birmingham, Birmingham, AL, USA; 2. Johns Hopkins Univ., Baltimore, MD, USA
}

Mutations in the cystic fibrosis transmemebrane conductance regulator (CFTR) gene are the cause of cystic fibrosis (CF). The deletion of phenylalanine at position 508 (F508del) is the most common CF mutation, and at least one copy is found in approximately $90 \%$ of $\mathrm{CF}$ patients. As such, rescue of the F508del CFTR mutation using small molecule approaches remains an important goal stimulating drug discovery and development. Success in this endeavor could have a considerable impact on $\mathrm{CF}$ outcomes. The F508del mutation primarily induces misfolding of CFTR, resulting in premature degradation of the protein prior to reaching the cell surface. The mutation also confers abnormalities in channel gating, thereby severely reducing ion transport activity even when the mutant protein resides at the cell surface. Knowledge of the molecular biology of the F508del defect suggests approaches to "correct" CFTR misprocessing may need to be combined with agents that "potentiate" CFTR gating to confer a meaningful clinical improvement (1).

In collaboration with the Cystic Fibrosis Foundation, Vertex Pharmaceuticals used high-throughput screening to evaluate hundreds of thousands of compounds in a cell-based screening platform designed to identify correctors of F508del processing and potentiators of CFTR activity (2). VX-770, an investigational agent and robust potentiator of CFTR function, increased short-circuit current in G551D/F508del primary human bronchial epithelial cells (HBE) to approximately $50 \%$ of activity observed in non-CF HBE (3). VX-770 also demonstrated in vitro activity in a subset of $\mathrm{HBE}$ cells derived from F508del CFTR homozygous individuals, suggesting that VX-770 can potentiate a small pool of F508del at the cell surface resulting in approximately $15 \%$ of non-CF HBE CFTR activity, prior to correction of F508del processing. VX-770 has completed Phase 2 testing and progressed through Phase 3 testing for the treatment of CF in individuals with the G551D gating mutation (4). Prolonged (48 week) treatment of CF subjects with at least one copy of G551D CFTR exhibited marked improvement in pulmonary function, a reduced chance of experiencing a CF pulmonary exacerbation, and a $\sim 45 \mathrm{mEq}$ improvement in sweat chloride, in addition to demonstrating an acceptable safety profile. In contrast, treatment of F508del homozygous CF patients with VX-770 was safe, but resulted in a small change in sweat chloride $(\sim 3 \mathrm{mEq})$ and did not alter clinical outcome; VX-770 treatment improved sweat chloride by $>10 \mathrm{mEq}$ in only a small subset $(\sim 15 \%)$ of F508del homozygous individuals. These results suggest that while demonstrating detectable bioactivity in some individuals, treatment with VX-770 alone is not sufficient as a single therapy in F508del homozygous subjects, and indicate the importance of correcting F508del processing in combination with a CFTR potentiator as also suggested by in vitro studies.

Using cell-based strategies, Vertex Pharmaceuticals and other laboratories have also discovered agents that correct F508del CFTR processing, resulting in rescued F508del protein at the cell surface and partially restored anion transport $(2,5-7)$. The lead corrector candidate in the Vertex program is the investigational agent VX-809, and in F508del homozygous HBE cells, VX-809 restored CFTR activity to $\sim 16 \%$ of non-CF HBE in vitro and was accompanied by improved band $\mathrm{B}$ to $\mathrm{C}$ formation, indicating the increased presence of CFTR residing at the cell-surface. In CF patients homozygous for F508del, sweat chloride improved $8 \mathrm{mEq}$ over placebo ( $7 \mathrm{mEq}$ overall) at the highest dose tested (200 $\mathrm{mg}$ daily) and demonstrated a reasonable safety profile. Unfortunately, 4 weeks of VX-809 monotherapy was not associated with a detectable clinical improvement in spirometry, suggesting bioactivity of VX-809 is not sufficient to improve clinical outcomes when used alone. When $\mathrm{VX}-770$ is added to VX-809 in vitro, anion transport activity doubles in F508del HBE cells, suggesting an additive effect when a potentiator is combined with a CFTR corrector. Interim results of the first CF clinical trial assessing the benefit of a corrector and potentiator in combination were recently reported. In this trial, two weeks of $200 \mathrm{mg}$ VX-809 treatment was followed by the addition $150 \mathrm{mg}$ or $250 \mathrm{mg}$ of $\mathrm{VX}-770$ twice daily for 7 days. Results demonstrated VX-770 further decreased sweat chloride compared to VX-809 alone (by $9 \mathrm{mEq}$ above VX-809 treatment alone at the VX-770 $250 \mathrm{mg}$ dose group and $13 \mathrm{mEq}$ (total change) following the combination of VX-809 and VX-770). These findings demonstrate the proof of concept that significant improvements in sweat chloride are possible upon combined treatment of F508del homozygous individuals 
with a CFTR potentiator and a CFTR corrector. Based on genotype-phenotype correlations in the disease, 13 $\mathrm{mEq}$ may be sufficient to confer improved clinical outcomes (8). Further testing of combination strategies involving CFTR correctors and potentiators are warranted in $\mathrm{CF}$, and may confer clinical improvement upon long-term testing in the most common mutation found in CF patients.

References: 1. Rowe SM, Miller S, Sorscher EJ. Cystic fibrosis. N Engl J Med. 2005;352:1992-2001.

2. Van Goor F, Straley KS, Cao D, et al. Rescue of DeltaF508-CFTR trafficking and gating in human cystic fibrosis airway primary cultures by small molecules. Am J Physiol Lung Cell Mol Physiol. 2006;290:L1117-30.

3. Van Goor F, Hadida S, Grootenhuis PD, et al. Rescue of CF airway epithelial cell function in vitro by a CFTR potentiator, VX-770. Proc Natl Acad Sci USA. 2009;106:18825-30.
4. Accurso FJ, Rowe SM, Clancy JP, et al. Effect of VX-770 in persons with cystic fibrosis and the G551DCFTR mutation. N Engl J Med. 2010;363:1991-2003.

5. Pedemonte N, Lukacs GL, Du K, et al. Small-molecule correctors of defective DeltaF508-CFTR cellular processing identified by high-throughput screening. J Clin Invest. 2005;115:2564-71.

6. Yang H, Shelat AA, Guy RK, et al. Nanomolar affinity small molecule correctors of defective Delta F508-CFTR chloride channel gating. J Biol Chem. 2003;278:35079-85.

7. Robert R, Carlile GW, Pavel C, et al. Structural ana$\log$ of sildenafil identified as a novel corrector of the F508del-CFTR trafficking defect. Mol Pharmacol. 2008;73:478-89.

8. Wilschanski M, Dupuis A, Ellis L, et al. Mutations in the cystic fibrosis transmembrane regulator gene and in vivo transepithelial potentials. Am J Respir Crit Care Med. 2006;174:787-94.

\title{
S16.1 \\ 28 DAY CHRONIC-INTERMITTENT INHALED ANTIBIOTICS: HOW DID WE GET THERE?
}

\author{
Arnold L. Smith, M.D. and Bonnie W. Ramsey, M.D. \\ Seattle Children's Hospital, University of Washington, Seattle, WA, USA
}

In the late 1970s, intravenous (IV) aminoglycosides, and particularly tobramycin, became an important part of the regimen for the management of CF pulmonary exacerbations. Aminoglycosides had much broader therapeutic indices than the polymyxins, while retaining excellent antipseudomonal activities. While studying the pharmacokinetics of IV tobramycin, we observed that tobramycin clearance in persons with $\mathrm{CF}$ was increased compared to healthy volunteers (1). Although aminoglycosides were known to be cleared from the systemic circulation in the urine, the glomerular filtration rate in persons with $\mathrm{CF}$ dramatically underpredicted their rate of aminoglycoside elimination (1). We speculated that the increased non-renal systemic clearance could be due to expectoration of the antibiotic in sputum. In order to improve aminoglycoside quantitation over the existing bioassay in complex matrices, such as sputum, we had developed radio-enzymatic assays (2) and were surprised to learn that levels of tobramycin in CF sputum during IV treatment were almost 10 times higher when assessed by RAE than by bioassay (3). Our observations suggested that clearance of (bound and inhibited) tobramycin by sputum expectoration could account for the accelerated tobramycin clearance in persons with CF (4). In addition, we were able to determine that sputum inhibition of tobramycin activity was saturable (suggestive of adsorptive bind- ing) and could be overwhelmed by addition of significantly higher levels of tobramycin. Our observation that CF sputum had a saturable inhibitory effect on tobramycin suggested that topical administration of high quantities of tobramycin by aerosol could overcome sputum inhibition, produce an active unbound fraction and result in improved tobramycin efficacy. Initially, our intention was to explore this treatment modality as a therapy to treat patients experiencing a pulmonary exacerbation.

While these studies were being conducted, we also completed a clinical trial of the synthetic penicillin azlocillin for the treatment of CF pulmonary exacerbations $(5,6)$. During that study, we recognized that a subset of our patients could be characterized as exacerbation "repeaters," meaning that they were always at an increased risk for an exacerbation, having many such episodes in a year. At the same time, other CF clinicians, and in particular physician-scientists in Denmark, had reported improved patient outcomes by use of scheduled antibiotic treatments in their patients (7). These observations led us to consider the merit of using scheduled "high dose" inhaled tobramycin as a prophylactic therapy to reduce risk of exacerbation in high risk patients.

When we began these investigations, aerosol generation was an uncharacterized route for chronic delivery of drugs, and particularly of drugs for which the potential for systemic toxicity was substantial. In order to 
better characterize any potential safety associated with prolonged administration, we completed an open-label, single arm 3-month study of inhaled tobramycin in 22 subjects (8). This was primarily a safety study to assess the effects of prolonged aerosol antibiotic administration on renal and 8th cranial nerve function; we meticulously tracked high-frequency audiometry, vestibular function, glomerular filtration rates and proximal renal tubular function in these subjects (8). There were no significant safety concerns with the treatment, and we observed that mean pulmonary function (assessed as the forced expiratory volume in 1 second; FEV1) increased during the first 4 weeks of treatment but then appeared to level off and even decrease during the following 2 months of treatment. In addition, nearly three quarters of patients had at least one P. aeruginosa isolate with an MIC above the tobramycin parenteral breakpoint at the end of 3 months of treatment (8). When respiratory secretions were assessed a month after cessation of treatment, the prevalence of tobramycin-resistant isolates had decreased dramatically (as had FEV1). This "reversion" of resistance phenotype in CF $P$. aeruginosa clinical isolates after removal of antibiotic pressure had been previously observed in studies of aminoglycosides, fluoroquinolones and beta-lactams (9-11), and was interpreted to mean that antibiotic resistance in $P$. aeruginosa could be "transient," and reversible.

In order to confirm the improvement in FEV1 observed in the 3-month inhaled tobramycin safety study, we subsequently conducted a randomized, placebo-controlled, taste-masked, cross-over study in which one group of patients received aerosolized high-dose tobramycin for 1 month followed by 2 months of placebo and the other group received 1 month of placebo followed by 2 months of inhaled tobramycin (12). This study confirmed a statistically significant improvement in FEV1 associated with tobramycin inhalation that (again) was not observed to increase beyond 4 weeks of treatment, and again showed no evidence of renal damage or neurotoxicity. In addition, although the protocol called for administration of inhaled tobramycin tid, review of adherence records and patient reporting indicated that less than $50 \%$ of midday doses had been administered during the study, suggesting that a) the observed benefits of inhaled tobramycin were likely to be retained with a bid dosing schedule and b) a tid dosing schedule was probably unrealistic given the delivery system being employed. By today's standards, it is understandable why the delivery system in use was problematic with respect to adherence: in order to receive a dose, a patient/parent had to dissolve $1.2 \mathrm{~g}$ of tobramycin sulfate in $30 \mathrm{~mL}$ saline, transfer $15 \mathrm{~mL}$ to an Ultraneb 100/99 ultrasonic nebulizer, add another $15 \mathrm{~mL}$ of saline, turn on the aerosol generator and let it run for 2 minutes to assure linear output, and then take 200 breaths (12). After use, the apparatus had to be rinsed and dried.
At this point, the potential benefit to the CF population of availability of a safe, effective, optimized formulation and delivery system for inhaled tobramycin was evident, but the cost of such an undertaking was daunting. The CF Foundation and Seattle Children's Hospital approached a small Seattle biotech company, PathoGenesis Corporation, to take on the task in return for rights to commercialization. We had collected evidence that measurable benefit of continuous treatment with inhaled tobramycin was maximized within 1 month, and that emergence of isolates with reduced tobramycin susceptibility was low 4 weeks after inhaled tobramycin administration had been discontinued, and thus the month-on/month-off schedule of inhaled tobramycin was born. In Phase 3 studies, inhaled tobramycin (now $300 \mathrm{mg}$ delivered bid by PARI $\mathrm{LC}+$ jet nebulizer) again showed an FEV1 benefit and relatively infrequent emergence of tobramycin-resistant isolates (13); the systematic study of $>500$ patients permitted the identification of an increased risk of tinnitus and hoarseness associated with this treatment (13).

In the intervening years since the validation of inhaled tobramycin as an effective chronic therapy, aerosol delivery systems have become faster, more efficient and more convenient. In addition, the expansion of molecular techniques and refinement in the study of $\mathrm{CF}$ microbiology has revealed that our assumptions regarding "transient" resistance and microbial ecology were overly simplistic.

Finally, tobramycin is no longer the only well-characterized inhaled antibiotic for management of persons with CF whose airway secretions yields $P$. aeruginosa, and even more inhaled antibiotics are in clinical development. Although other inhaled antibiotics have been and will be studied independently in placebo-controlled trials, optimal management of our patients will likely be achieved by coordinated use of these therapies. Thus, the next phase of this journey is to consider how best to study their use together.

References: 1. Levy J, Smith AL, Koup JR, et al. J Pediatr. 1984;105:117-24.

2. Weber A, Smith AL, Opheim KE. J Clin Microbiol. 1985;21:419-24.

3. Mendelman PM, Smith AL, Levy J, et al. Am Rev Respir Dis. 1985;132:761-5.

4. de Groot R, Smith AL. Clin Pharmacokinet. 1987;13:228-53.

5. Woolf RA, Koup JR, Smith AL, et al. Clin Pharm. 1985;4:664-9.

6. Smith AL, Doershuk C, Goldmann D, et al. J Pediatr. 1999;134:413-21.

7. Hoiby N, Jacobsen L, Jorgensen BA, et al. Acta Paediatr Scand. 1974;63:843-8.

8. Smith AL, Ramsey BW, Hedges DL, et al. Pediatr Pulmonol. 1989;7:265-71.

9. Karlowsky JA, Saunders MH, Harding GA, et al. Antimicrob Agents Chemother. 1996;40:1387-93. 
10. Giwercman B, Lambert PA, Rosdahl VT, et al. J Antimicrob Chemother. 1990;26:247-59.

11. Chamberland S, Malouin F, Rabin HR, et al. J Antimicrob Chemother. 1990;25:995-1010.
12. Ramsey BW, Dorkin HL, Eisenberg JD, et al. N Engl J Med. 1993;328:1740-6.

13. Ramsey BW, Pepe MS, Quan JM, et al. N Engl J Med. 1999;340:23-30.

\title{
S16.2 \\ IS CHRONIC-INTERMITTENT DOSING OF INHALED ANTIBIOTICS THE OPTIMAL STRATEGY? A EUROPEAN PERSPECTIVE
}

\author{
Steven P. Conway, MBBS, FRCP, FRCPCH \\ Cystic Fibrosis Unit, St. James Hospital, Leeds, United Kingdom
}

Imagine the scenario of the $\mathrm{CF}$ doctor explaining inhaled antibiotic protocols to a non-medical person:

Doctor: So, we administer the newer inhaled antibiotics for four weeks and then stop treatment for 4 weeks. This cycle is repeated ad infinitum.

Other: Only the newer antibiotics?

Doctor: Yes. Although since the 1980s in Europe we have administered other antibiotics every day with no drug free interval.

Other: Does the patient's condition improve during the treatment month?

Doctor: Yes.

Other: Does the patient's condition get worse during the month off treatment?

Doctor: Yes.

Other: So why do you stop the treatment?

Doctor: To encourage adherence, reduce toxicity and prevent the development of bacterial antibiotic resistance.

Other: And....

Doctor: Adherence remains poor. The drugs appear safe. Resistance as defined for drugs given intravenously probably has no relevance to inhaled antibiotics.

Other: Right!

Let us review the efficacy of daily inhaled antibiotic therapy. Hodson showed that daily nebulised antibiotics improved respiratory function and reduced the incidence of hospital admissions(1). Subsequent studies show a slower decline in respiratory function, improved clinical scores and weight, decreased $P$. aeruginosa density, exotoxin A, or elastase(2). These studies are weakened by small patient numbers and the use of varied antibiotics at varied doses administered by varied delivery systems, but meta-analyses confirm positive treatment effects(3). No see-saw clinical responses were observed.

The first evidence based study of inhaled antibiotic treatment used a 4-week alternating "on/off" regimen for Tobramycin Solution for Inhalation (TSI)(4). Because of the vogue for evidence based medicine - "The use of cyclic, suppressive inhaled antibiotic therapy with 28 days of therapy followed by 28 days off therapy has become the standard of care for CF patients $>6$ years of age with chronic $P$. aeruginosa infection"(5) - the on/off TSI regimen has become the comparator for all trials of new medications, despite the fact that in that(4) and every subsequent study, patients show a roller coaster response (up in the "on" months, down in the "off" months), challenging the claim that improvement in lung function was maintained during each "off" period(4).

I suggest there is no rationale for allowing patients to deteriorate every other month and that the reasons promulgated for alternate month therapy are flawed. A challenge to a continuous daily regimen should show at least lack of inferiority. This has not been addressed or proven. Instead we chart the "month off" fall in respiratory function and quality of life (QoL) or, by a variety of interventions, nibble at the on/off approach so that patients may take therapy only once daily but continuously, or take an alternative inhaled antibiotic in the "off" month. The result? None of us knows what constitutes optimal inhaled antibiotic treatment. I suggest that at least in part we have accepted the on/off regimen as a baseline for treatment not so much because of fears of drug toxicity and bacterial resistance, but because halving the annual cost makes the prescription more palatable to those who pay.

Arguments for on/off treatment plead the case for better adherence, less toxicity risk, less probability of developing increased bacterial antibiotic resistance, but where is the evidence? Patients renege on significant parts of treatment regimens, in particular chest physiotherapy and aerosolised antibiotic protocols because these are time consuming and without immediate gratification. We have shown a median adherence of $36 \%$, measured by nebuliser download(6). Rather than argue that an on/off regimen will increase adherence, (hypothetical, not evidence-based), we should argue that with such a protocol and known adherence patterns patients are likely to be in receipt of their prescribed aerosolised antibiotic for less than 2 out of every 8 weeks. Positive results seen during clinical trials probably reflect the stimulus effect of taking part in the study and bias possibly introduced by the patients agreeing to enrol being those most motivated to take their treatment. Even so every study of on/off treatment shows a fall towards 
baseline values in the "off" month. How much more pronounced will this be in the reality non-clinical trial situation of poor adherence? In practice patients probably achieve the equivalent of an on/off regimen when prescribed continuous treatment!

What is the likelihood of drug toxicity? There are no data for long term continuous use of inhaled TSI or aztreonam (AZLI) but safety data available are reassuring. A review of studies from 1965-95(7) and a 1999 Consensus Conference (8) show no evidence of renal or ototoxicity. Extra vigilance is needed for patients with renal insufficiency (9) and with the more efficient mesh based nebulisers, although the risk is low(10). Good safety data are published for tobramycin inhaled powder (TIP)(11) and AZLI(5).

Is bacterial resistance with continuous daily inhaled therapy likely to be a practical issue? There are no longterm data for the newer antibiotics but despite the widespread use of twice daily nebulised colistin in Europe, resistance has not been a problem. Reassuringly, studies of on/off therapy with TSI, TIP, and AZLI have not shown significant increases in resistance to the inhaled preparation, increased resistance to other antibiotics, or increased isolation of naturally resistant bacteria, e.g. B. cepacia complex, Stenotrophomonas, Achromobacter. Moreover, "susceptible thresholds for parenteral administration might not be relevant to inhaled therapy as the drug concentration achieved at the site of infection with inhaled antibiotics, including TSI, can be significantly higher than the systemic concentration"(12), and the clinical response appears to be independent of the MIC(13). Indeed, Moss advises that the argument about increased bacterial resistance is less important than addressing the progressive fall in lung function that accompanies $P$. aeruginosa infection(13).

In conclusion we must ask why we wish to risk compromising patients' immediate well-being and longevity by only treating them half the time if we accept that:

1) inhaled antibiotic therapy results in a significant mean improvement in lung function and QoL, a mean decrease in sputum $P$. aeruginosa density that significantly correlates with increases in FEV1(13), and reduced hospital admissions;

2) patients are likely to suffer an annual loss of lung function of $1-4 \%$ and that TSI may impact on the natural course of the disease by reducing this rate of fall(14);

3) small airways disease starts early, is only detectable by standard spirometry when substantial obstruction has occurred, is exacerbated by $P$. aeruginosa acquisition, and improved by TSI therapy(15);

4) a delay in starting inhaled antibiotic treatment is associated with an impaired response suggesting an irreversible component to lung function decline(13);

5) patients will have recurrent respiratory infective exacerbations resulting in long term decline in FEV1, especially in children so that "preventing exacerbations may ultimately be more important than the approach taken to treat the exacerbation"(16);
6) only treating exacerbations will not arrest the progressive decline in lung function(17); and

7) most patients will not be more than $50 \%$ adherent to inhaled treatment regimens.

The argument for an alternating four week on/off treatment regimen has to be a solecism.

References: 1. Hodson ME, et al. Aerosol carbenicillin and gentamicin treatment of Pseudomonas aeruginosa infection in patients with CF. Lancet. 1981;2:1137-9.

2. Conway SP. Evidence for using nebulised antibiotics. Arch Dis Child. 1999;80:307-9.

3. Mukhopadhyay S, et al. Systematic review of nebulised antibiotics in cystic fibrosis. J R Soc Med. 1998;91(S34):25-9.

4. Ramsey BW, et al. Intermittent administration of inhaled tobramycin in patients with CF. N Engl J Med. 1999;340:23-30.

5. Oermann CM, et al. An 18 month study of the safety and efficacy of repeated courses of inhaled aztreonam lysine. Pediatr Pulmonol. 2010;45:1121-34.

6. Daniels T, et al. Accurate assessment of adherence. Chest. 2011;140:425-32.

7. Touw DJ, et al. Inhalation of antibiotics in CF. Eur Respir J. 1995;8:1594-604.

8. Campbell PW 3rd, et al. Use of aerosolised antibiotics in patients with cystic fibrosis. Chest. 1999;116:775-88.

9. Edson RS, et al. Vestibular toxicity due to inhaled tobramycin in a patient with renal insufficiency. Mayo Clin Proc. 2004;79:1185-91.

10. Guy E, et al. Serum tobramcyin levels following delivery of tobramycin via Eflow advanced nebuliser. J Cyst Fibros. 2010;9:292-5.

11. Konstan MW, et al. Tobramycin inhalation powder for $P$. aeruginosa infection in cystic fibrosis. Pediatr Pulmonol. 2011;46:230-8.

12. Burns JL, et al. Effect of chronic intermittment administration of inhaled tobramycin on respiratory microbial flora. J Infect Dis. 1999;179:1190-6.

13. Moss RB. Long-term benefits of inhaled tobramycin in adolescent patients. Chest. 2002;121:55-63.

14. Van Devanter D, et al. Effect of TOBI® on longterm rates of lung function decline in $P$. aeruginosa infected CF patients with mild to moderate lung disease. Pediatr Pulmonol. 2000;S20:298.

15. Tiddens HA, et al. Cystic fibrosis lung disease starts in the small airways. Pediatr Pulmonol. 2010;45:107-17.

16. Sanders DB, et al. Pulmonary exacerbations are associated with subsequent FEV1 decline. Pediatr Pulmonol. 2011;46:393-400.

17. Quan JM, et al. Treatment for exacerbations only does not arrest progressive lung function decline in $\mathrm{CF}$. In: Heijerman HGM, et al., ed. 23rd European CF Conference. The Hague:Elsevier,1999:S84. 


\title{
S16.3 BEYOND INHALED MONOTHERAPY: HOW DO WE MEASURE SUCCESS?
}

\author{
Donald VanDevanter, Ph.D. \\ Pediatrics, Case Western Reserve School of Medicine, Edgewood, WA, USA
}

We have entered a new age with respect to inhaled antibiotic therapies for the management of patients with $\mathrm{CF}$ and chronic Pseudomonas aeruginosa lung infections. Members of two different antipseudomonal antibiotic classes (aminoglycosides and monobactams) have now been specifically formulated for inhalation and studied extensively in large controlled clinical trials (1-4). The commercial availability of these agents and the likely regulatory approval of other inhaled antibiotics in the near future creates an opportunity for use of these agents "in concert" in the management of individual patients. Although there may be an expectation that use of multiple inhaled antibiotics can produce better outcomes than monotherapy, there is and will be little objective evidence of improved outcomes for combining these therapies derived from registration studies. Rather, it will likely be up to the CF community to determine how to best combine these therapies and whether resulting outcomes are "better."

There are a number of outcomes that have been used to study antibiotic therapies, all of which could potentially support studies of inhaled antibiotic combinations. These include clinical endpoints such as effect on pulmonary exacerbation, quality of life, and weight gain, as well as the surrogate endpoint of pulmonary function assessed by spirometry (5) and "markers" of benefit such as change in high-resolution computerized tomography (HRCT) (6). Other marker endpoints that would lend themselves to studying chronic antibiotics include lung clearance index (LCI) by multiple-breath washout (7), and markers of inflammation in respiratory secretions (8). Emergence of local and systemic toxicities and changes in infection microbiology have been employed to study safety issues (1).

There will be challenges in designing trials to demonstrate an additional benefit of combining inhaled antibiotics, including powering of a superiority endpoint using an active comparator (inhaled monotherapy), maintenance of blinding when each aerosol treatment has a different (and identifiable) formulation and delivery device, differences in patient response that may result from infection history, airway microbial community, and previous antibiotic exposures, and choice(s) of treatment rotation schedule. While these are difficult challenges, they seem tractable and it is likely that a study comparing inhaled antibiotic monotherapy (or monotherapies) to combination therapies could be designed.

More important than design questions is the discrepancy between the likely duration of even the most ambitious randomized, controlled, prospective trial (a few months to a year?) and the length of time that a patient with a chronic lung infection might benefit from chronic suppressive antibiotic therapy (perhaps decades). Funda- mental clinical questions are likely to remain unanswered upon successful completion of a 6 month or even 1 year study comparing inhaled monotherapy to combination therapy. How would clinicians extrapolate risks and benefits identified in such a study to extended use of inhaled antibiotics? How likely is it that the study would have recognized and addressed the underlying motivation for combining inhaled antibiotic therapies in the first place? Is this really about increased amplitude of benefit, or rather increased duration of benefit?

It is difficult to find researchers or caregivers who do not intuit that combination of inhaled antibiotic classes should produce better outcomes than monotherapy over the lifespan of a patient with CF. Why is this the case and what assumptions underlie this belief? One assumption (A) appears to be that there is a finite capacity for any single antibiotic to produce benefit when administered chronically to a patient with a chronic lung infection: at some point, a patient will become refractory to response with that antibiotic. This seems a rational and reasonable assumption. A companion assumption (B) is that this capacity for benefit can be manipulated by variation of antibiotic dose, schedule and regimen. This is the underlying tenet of chronic intermittent inhaled tobramycin: continuous use of inhaled tobramycin for 3 months showed decreasing pulmonary function benefit after 1 month (9), while chronic intermittent inhaled tobramycin use showed continued efficacy after 6 months (1) to two years (10). This construct has never been objectively tested in a single study and these historical studies differ in important ways (e.g., dose, dose schedule, delivery device, sample size). However, this assumption also seems reasonable.

The promise of an additional benefit derived from a second inhaled antibiotic can be found in both assumptions (A) and (B), above. If assumption (A) is true, then access to 2 antibiotics increases the amount of time that a patient can experience the benefit of chronic inhaled antibiotic suppression compared to the situation in which that patient has access to only monotherapy. This relative benefit is unlikely to be demonstrated in a 1-year prospective study, since we already know that the benefits of inhaled antibiotic monotherapy can extend beyond 1 year $(10,11)$. If assumption (B) is true, then there may be a way to "rotate" the 2 inhaled antibiotics such that the effective duration over which each antibiotic provides benefit exceeds the total benefit that would be achieved if each antibiotic was used successively as a monotherapy. This is all very well and good and may even be true. However, this "benefit" of inhaled antibiotic rotation (extending the effective life of each chronic inhaled 
antibiotic) would presumably play out over the course of years or even decades of a patient's life, and is unlikely to be captured by the endpoints we are considering in relatively short (6-12 month) controlled studies.

If the true promise of inhaled antibiotic rotation lies in delaying that time at which a patient becomes refractory to all available inhaled antibiotic therapies, then we have yet to consider a clinical trial endpoint likely to capture that benefit. Shorter term endpoints may justify or encourage combinational antibiotic use by clinicians, but they will not allow us to answer this greater question: have we increased the effective time span that we can treat patients? Not that we are unable to measure refractoriness...it is actually quite simple. When an individual becomes refractory to a chronic therapy that he or she had previously responded to, then treatment cessation should not result in immediate clinical deterioration. Our challenge is that we already know that most patients remain responsive to chronic intermittent inhaled antibiotic monotherapy for more than a year, as evidenced by consistent, reproducible declines in $\mathrm{FEV}_{1}$ and QoL observed during their off-drug periods $(10,11)$. Thus, choosing a "time to refractoriness" endpoint for a study of a year or less duration would be misguided, at best.

In conclusion, although prospective randomized studies comparing the superiority of combining inhaled antibiotics to monotherapy are feasible, they are unlikely to address underlying motivations (and greatest potential benefits) of combining therapies: extension of the effective time during which infections can be suppressed. Rather, it will be the job of providers to consider whether patients under their care continue to benefit from inhaled antibiotics (rotated or not) after months and years of use, to consider careful and deliberate $\mathrm{N}$ of 1 "withdrawal studies" to assess refractoriness, and to record these data in a manner that will allow us to characterize outcomes using retrospective, observational analyses.
References: 1. Ramsey BW, Pepe MS, Quan JM, et al. Intermittent administration of inhaled tobramycin in patients with cystic fibrosis. Cystic Fibrosis Inhaled Tobramycin Study Group. N Engl J Med. 1999;340:23-30.

2. Murphy TD, Anbar RD, Lester LA, et al. Treatment with tobramycin solution for inhalation reduces hospitalizations in young $\mathrm{CF}$ subjects with mild lung disease. Pediatr Pulmonol. 2004;38:314-20.

3. Retsch-Bogart GZ, Quittner AL, Gibson RL, et al. Efficacy and safety of inhaled aztreonam lysine for airway Pseudomonas in CF. Chest. 2009;135:1223-32.

4. McCoy KS, Quittner AL, Oermann CM, et al. Inhaled aztreonam lysine for chronic airway $P$. aeruginosa in cystic fibrosis. Am J Respir Crit Care Med. 2008;178:921-8.

5. Ramsey BW, Boat TF. Outcome measures for clinical trials in CF. Summary of a Cystic Fibrosis Foundation consensus conference. J Pediatr. 1994;124:177-92.

6. Nasr SZ, Sakmar E, Christodoulou E, et al. The use of high resolution computerized tomography of the chest in evaluating the effect of tobramycin solution for inhalation in cystic fibrosis lung disease. Pediatr Pulmonol. 2010;45:440-9.

7. Aebischer CC, Kraemer R. The lung clearance index (LCI) as an estimate of ventilation inequalities in patients with cystic fibrosis. Agents Actions Suppl. 1993;40:73-83.

8. Sagel SD, Chmiel JF, Konstan MW. Sputum biomarkers of inflammation in CF lung disease. Proc Am Thorac Soc. 2007;4:406-17.

9. Smith AL, Ramsey BW, Hedges DL, et al. Safety of aerosol tobramycin administration for 3 months to patients with CF. Pediatr Pulmonol. 1989;7:265-71.

10. Moss RB. Long-term benefits of inhaled tobramycin in adolescent patients with cystic fibrosis. Chest. 2002;121:55-63.

11. Oermann CM, Retsch-Bogart GZ, Quittner AL, et al. An 18-month study of the safety and efficacy of repeated courses of inhaled aztreonam lysine in cystic fibrosis. Pediatr Pulmonol. 2010;45:1121-34.

\title{
S16.4 CHANGING THE PARADIGM: HOW DO WE FIGURE THIS OUT?
}

\author{
Patrick A. Flume, M.D. \\ Medical Univ. of South Carolina, Charleston, SC, USA
}

Today, the standard dosing interval for FDA-approved inhaled antibiotics is 28 days, with an implied 28 day rest period. This regimen was originally conceived based upon interpretation of results from clinical studies with inhaled tobramycin conducted decades ago, but never objectively tested against other possible regimens. The availability of multiple classes of inhaled antibiotics allows the clinician to consider a number of approaches for combining different inhaled antibiotics into a single management plan. How will we go about identifying those approaches that are the most likely to provide improved outcomes for the population?
The strategy of intermittent (e.g., month on-month off) antibiotic dosing has been contested in clinical practice. This has been done primarily because the clinician and patient have decided that there are unacceptable outcomes associated with that regimen, such as loss of lung function or occurrence of pulmonary exacerbations. Indeed, in the original trials of inhaled tobramycin, $40 \%$ of patients on tobramycin required treatment for an exacerbation (1). Some may argue that the reduction in lung function observed in the "off" months demonstrates worsening infection and inflammation and more aggressive intervention 
may prevent further loss of lung function. In these cases, the patient would have been treated by either adding a second drug, usually an off-label intravenous antibiotic (e.g., colistin), or continuous use of the inhaled tobramycin (2).

Today there are two inhaled antibiotic formulations FDA-approved for treatment of persons with cystic fibrosis (CF) and Pseudomonas, and several other agents in latestage clinical trials. If all goes according to plan, clinicians will have the "luxury" of being able to prescribe one or more of several classes of anti-pseudomonals in the near future. Although FDA approval will have required demonstration of efficacy of an inhaled antibiotic compared to a placebo, and there will be some head-to-head data available as well, what clinicians will really need is some indication as to whether management of our patients will benefit from using these drugs in some sort of combination, as opposed to as single agents. One can propose a number of approaches for combining different inhaled antibiotics into a single management plan (Table). Presumably, some approaches are more justifiable from a mechanistic standpoint than others. Similarly, some are likely to be more feasible from a treatment burden, adherence, and/or cost perspective. How will we go about identifying those approaches that are the most likely to provide improved outcomes for the population?

The two most basic questions involve a comparison of intermittent (e.g., month on-month off) against continuous (no time off) therapy (Table-Strategy 1 vs. Strategy 3 ) or a comparison of a combination (or rotation) of antibiotics compared to a single drug (Table-Strategy 2 vs. Strategy 3 ). The addition of more antibiotics increases the complexity even further as we can then consider intermittent therapy using a rotation of antibiotics.

The design of treatment strategies for a trial is not difficult; several are shown in the Table, but the challenge lies in the efficacy endpoints that would help us know which treatment strategy is the most effective.

Whereas previous studies of aerosol antibiotics have demonstrated improvement in $\mathrm{FEV}_{1}$ and quality of life, and a reduction in exacerbations, these were short-term trials powered based upon comparison against placebo. How would those endpoints work in a comparison against another inhaled antibiotic, one of which has been in use for a long period of time?

As noted earlier, the suggestion that a different treatment strategy should be considered is based upon the observation that the current FDA-approved strategy of intermittent therapy is not optimal. So it would seem that important clinical parameters that prompt a change in treatment approach to either continuous therapy or rotation of antibiotics (or both) include loss of lung function (i.e., over time), impaired quality of life, and frequent exacerbations. These, too, would not be difficult endpoints to measure in a clinical trial, but the feasibility of performing such trials is challenging because the studies would likely require longer durations (i.e., it is unlikely that we would see a meaningful difference in the short term) and power analyses suggest that large numbers of patients would be required.

There are additional challenges to the feasibility of performing such trials. The current antibiotic choices use different drug-device combinations and it may be impossible to completely blind patients and investigators to the treatment. A double-blind, double-dummy approach to circumvent this problem may be unrealistic. Thus, an open-label design may be the best approach, even with the potential for bias. What is the impact of previous antibiotic exposure? This could be accounted for by randomization of patients based on prior usage, but the addition of randomization factors results in a greater number of patients required for the study.

Finally, how would such a study be funded? Industry is required to demonstrate the efficacy and safety of their drug being studied; they are not required to demonstrate the optimal treatment strategy, but typically follow established paradigms under FDA guidance. Comparative effectiveness trials using the CFF Patient Registry seems the most likely avenue but we cannot expect third party payors to be interested in paying for the added medications in the absence of existing evidence. It seems a partnership between the CFF, industry, the FDA, and some third party payors would be necessary to perform such a trial.

References: 1. Ramsey BW, Pepe MS, Quan JM, et al. Intermittent administration of inhaled tobramycin in patients with cystic fibrosis. $N$ Engl J Med. 1999;340:23-30.

2. Nichols PL, Gray SL, Flume, PA. Usage of tobramycin solution for inhalation in an adult cystic fibrosis center. Am J Respir Crit Care Med. 2000;161:A72.

Potential Strategies of Aerosol Antibiotic Use

\begin{tabular}{lllllllllllll}
\hline & \multicolumn{10}{c}{ Month } \\
\hline Strategy & 1 & 2 & 3 & 4 & 5 & 6 & 7 & 8 & 9 & 10 & 11 & 12 \\
1 & A & & A & & A & & A & & A & & A & \\
2 & A & B & A & B & A & B & A & B & A & B & A & B \\
3 & A & A & A & A & A & A & A & A & A & A & A & A \\
4 & B & B & B & B & B & B & B & B & B & B & B & B \\
5 & A & & B & & A & & B & & A & & B & \\
\hline
\end{tabular}




\title{
S17.1 \\ DISCUSSING END OF LIFE CARE PLANNING; WHEN AND HOW?
}

\author{
Mark S. Pian, M.D. \\ Rady Children's Hospital \& Health Center, UC San Diego, San Diego, CA, USA
}

This presentation addresses the following objectives:

1. Establish goals of introducing end of life care discussions in the plan of care for $\mathrm{CF}$ patients and families. As a result of recent data suggesting efficacy for treatments attacking basic defects in mutant CFTR function, optimism for finding a cure for cystic fibrosis is at its highest level since the identification of the CFTR gene (and its accompanying promise of gene therapy). However, patients and families still live with the reality of a disease that continues to impose the need for choice from among a wide range of palliative and aggressive treatments as the illness progresses and premature death approaches. Careful integration of end of life discussions, with goals appropriate to the current condition and disease trajectory of individual patients, can help patients and families navigate these choices deliberately, with confidence, and with hope toward the best possible outcomes.

2. Identification of challenges associated with discussing end of life care with CF patients and their families. Traditionally, care teams have adopted strategies that have been successful in helping patients and families to accept aggressive and intensive monitoring and intervention as necessary parts of their lives. Enthusiastic and encouraging emphasis on the benefits to be achieved, and reinforcement of behavior on the part of families that reflects the same enthusiastic optimism is an important part of this approach. Though this approach has been important in translating the introduction of new therapies into better FEV1s, higher BMIs and longer lives, it can also send the unwanted message that fears regarding premature death and progressive illness themselves endanger the best outcomes, and are not appropriate topics to bring up with the team. Conversely, inviting such discussions can be seen by the care team and family as "taking away hope," depriving patients and those caring for them of powerful tools that can address directly such fears and, in many cases, allay them.

3. Identification of "triggers" signaling the need for, and opportunities to introduce, end of life care discussions. Impending death, or refusal to undergo lung transplantation with progressive severe lung dysfunction, certainly signals the need for end of life discussions. However, patients signal their need for such discussions, and their opportunity to benefit from them, earlier in their course. Teams can learn to recognize these signals and how best to respond to them.

4. Examination of differences in end of life discussions at different stages of life and disease progression. Though end of life discussions surrounding impending death are less frequent in children than they are in adults, they still occur. Of equal importance are concerns of children and their families regarding how the threat of shortened life span may affect quality of life, adherence to treatment and normal development in patients who are far from respiratory failure or decisions regarding transplant.

\section{S17.2 \\ THE ROLE OF THE MULTIDISCIPLINARY TEAM IN THE CARE OF THE CF PATIENT AT END OF LIFE}

\author{
Jessica L. Goggin, R.N., B.S.N. ${ }^{1}$ and Jennifer G. Rounds, R.N., B.S.N. ${ }^{2}$ \\ 1. Univ. of California San Diego Medical Ctr., San Diego, CA, USA; 2. Univ. of Nebraska Medical Ctr., Omaha, NE,
} USA

The multidisciplinary team has become the cornerstone in the delivery of cystic fibrosis $(\mathrm{CF})$ care and is well understood by team members, patients, and caregivers. Multidisciplinary team members contribute to the high quality of care that has come to be expected in Cystic Fibrosis Foundation Accredited Care Centers and are essential in the care of the CF patient at end of life. Unfortunately, not all members of the multidisciplinary team fully understand their roles and responsibilities in end of life or palliative care of CF patients. To better assess team members' understanding of roles and educational exposure in caring for CF patients at end of life a survey was developed and distributed via the established list serv's within the CF community. The survey was distributed to 244 nurses, 421 dietitians, 423 social workers and 192 respiratory therapists. Responses were received from 45 (18\%) nurses, 102 (24\%) dietitians, 57 (13\%) social workers, and $51(27 \%)$ respiratory therapists. The results of the survey indicate that although the majority of respondents have 15 or more years of experience within their discipline, most have been working with $\mathrm{CF}$ patients for 5 years or less. The majority of respondents or $44 \%$ reported they "somewhat understand" their role in caring for $\mathrm{CF}$ patients at the end of life, with social workers feeling most confident in their role and dietitians feeling least confident. All disciplines expressed the 
need for additional education related to advanced care planning and care of the CF patient at end of life. This information is presented in an initial effort to assist multidisciplinary team members to recognize and understand their respective roles in end of life care of patients with $\mathrm{CF}$ and will examine the roles of the nurse, social worker, respiratory therapist, and dietitian.

The role of the CF nurse may differ slightly depending upon whether or not care is provided in primarily an inpatient or an outpatient setting. Regardless of the setting the CF nurse works, similar principles should be used in providing end of life care. Four guidelines that can be used by the CF nurse in the care of the patient at the end of life and in advanced care planning are 1) providing the patient and family with support at the end of life or in advanced care planning; 2) supporting informed discussions about care goals and treatment options; 3 ) assisting the patient by facilitating the implementation of the patient's wishes regarding end of life care; and 4) knowing and understanding current legal issues surrounding end of life care and advanced care planning (1). The CF nurse may also need to assure that the patient and family are comfortable with decisions that have been made, and if not, may need to refer them to appropriate sources such as the palliative care team, social work, or psychology as appropriate. As is true in virtually all aspects of nursing, often the nurse spends the most time with the patient and family. This places the nurse in a position to identify needs of the patient and family and to address these needs or communicate them to the appropriate team members. It is through these roles, along with effective communication skills that the CF nurse is able to provide effective care at end of life.

Survey results indicate that social workers are most familiar and comfortable with their discipline's role in caring for patients during end of life. This is not unexpected as social workers are commonly consulted at these difficult times. The role of the CF social worker in the care of the CF patient at the end of life and in advanced care planning is consistent with the role in caring for any patient at the end of life, or in advanced care planning. However, the unique, often long-term relationship of the $\mathrm{CF}$ social worker with the $\mathrm{CF}$ patient may provide a strong foundation of support to the patient and family in dealing with sensitive end of life issues. According to the National Association of Social Workers Standards for social work practice in palliative care and end of life, there are several standards that social workers can use to help them work more effectively in end of life settings. Some of those standards include 1) Ethics and Values; 2) Knowledge; 3) Assessment; 4) Intervention/Treatment Planning; 5) Attitude/Self-Awareness; 6) Empowerment and Advocacy; 7) Interdisciplinary Teamwork; and 8) Cultural Competence (2). Through following and utilizing these standards in everyday practice social workers can be very effective in helping the CF patient and their family at end of life and at the time of advanced care planning.

Respiratory therapists are essential members of the $\mathrm{CF}$ multidisciplinary team across the continuum of care, including care delivered towards the end of life. Although approximately $80 \%$ of respiratory therapists report that they "somewhat" or "fully" understand their role in end of life care of CF patients, $50 \%$ feel minimally or not at all prepared to deliver that care. The role of the respiratory therapist extends across the outpatient and inpatient settings and includes participation in family conferences regarding goals of care, management of invasive and noninvasive ventilation support, and education regarding appropriate use and delivery of airway clearance therapy during end of life. Respiratory therapists may be asked to deliver hands-on chest physiotherapy toward end of life due to increased effectiveness or additional contact provided by the respiratory therapist (3). The respiratory therapist often spends a significant amount of time at the bedside for delivery of nebulized medications and airway clearance therapy and often develops close relationships with patients and families. The value of these close, often long-term relationships should not be underrated and may be a significant asset during discussions about end of life and treatment preferences.

Dietitians are integral members of every CF multidisciplinary team. In the survey, dietitians reported the least understanding of their role in end of life care. This lower level of understanding is not surprising as $99 \%$ of dietitians also responded that they have received less than 10 hours end of life education in CF. Nutrition is an essential component of CF disease management and remains a necessary area of focus during end of life as well. Patients with $\mathrm{CF}$ have long been taught to maintain high calorie, high protein diets due to pancreatic malabsorption and many older patients also have co-morbidities of CF-related diabetes (CFRD). Maintenance of BMI has been a goal of most CF patients (or at least of their dietitians) across their life span and nutritional issues at end of life may be complicated by this lifelong effort.

The multidisciplinary team is essential to the care of $\mathrm{CF}$ patients across the lifespan, including toward the end of life. The established team provides an ideal environment for delivering education to ensure that each clinician fully understands their role and is adequately prepared to care for the CF patient and their family toward end of life.

References: 1. Stilos K, Daines P. Guiding decisions about end-of-life care: Navigating the nursing role. CANNT J. 2010;20:56-7.

2. National Association of Social Workers. Standards for social work practice in palliative and end of life care. 2004.

3. Flume PA. Pulmonary complications of cystic fibrosis. Respir Care. 2009;54:618-27. 


\title{
S17.3 \\ INTEGRATING PALLIATIVE CARE INTO THE CF CARE MODEL
}

\author{
Walter M. Robinson, M.D., M.P.H. \\ Center for Applied Ethics, Education Development Center, Newton, MA, USA
}

A distinctive chronic care model, with an emphasis on prevention, routine clinical monitoring, early intervention, diagnostic standardization, and multi-disciplinary care, was introduced into US CF care in the 1960s and 1970s. Improved survival attributed to this model of care led to its widespread adoption in cities across North America. Accreditation of care centers by the US CFF promoted the model as the standard of care for cystic fibrosis. As the CF population aged, the model was adopted by centers caring for adults, with some important modifications. Monitoring of local outcome data was made routine with the widespread adoption of the CFF Clinical Registry, and was later expanded by the CFF using well-known quality improvement methods. Details of the CF care model are now routinely cited as examples of efficient and effective care that could be applied to other clinical conditions.

A specific model for the care of the dying patient in North America began almost simultaneously. Drawing on a shift in American popular concern about the "medicalization" of dying, and utilizing clinical models developed in the UK and elsewhere, a system of care for those with terminal illness slowly gained strength in the U.S. and Canada. Initially based outside hospitals and using the principles of hospice, this shift in clinical care for the dying gained strength in the 1990s as both clinicians and patients began to recognize the need for better symptom control in life-limiting illness. The increasing use of supportive technologies, including those formerly limited to intensive care units, and the development of new therapies with both high risk and great potential for benefit meant that a greater proportion of deaths in the US occurred after an aggressive but ultimately futile intervention. The palliative care model, distinct in both location and principles from the hospice model, began to emerge in clinical centers across the continent. This model developed unevenly in pediatrics, but grew rapidly in adult medicine. The palliative care model emphasized control of symptoms (even if the cause of the symptoms could not be eliminated), management of both treatment and disease side effects, shared decision-making with patients and families, recognition of the social and spiritual aspects of life-limiting illness (which many perceived to be lost in an increasingly bureaucratized and depersonalized death), and above all, effective communication with patients and families about prognosis and treatment options.

Integration of these two care models - the CF chronic disease model and the palliative care model — can be difficult for a host of reasons, yet there are also factors distinctive to the care of children and adults with $\mathrm{CF}$ that can promote better palliative care.

\section{Barriers to Effective Palliative Care in Cystic} Fibrosis.

1. Lack of CF-specific symptom research. Studies performed over the last decade have amply demonstrated the breadth and depth of the symptom burden in $\mathrm{CF}$, but few studies have examined specific treatments for those with CF.

2. Lack of systematic study of CF care in the last three months of life. There is a scarcity of up-to-date, multicenter, prospective observational studies of the clinical and social characteristics of death due to $\mathrm{CF}$, such as medications used and their efficacy, patterns of care, moral and ethical distress of caregivers, patients and families, and a host of other factors. Such studies are widely performed in oncology, and the methods used in oncology could easily be adapted to gain clinical insights into the relative success and failure of specific approaches to $\mathrm{CF}$ care in the last three months of life.

3. An "either/or" attitude to curative and palliative care among CF clinicians. The CF clinical care model emphasizes close adherence to a difficult regimen; we rightly emphasize that adherence can extend life, often dramatically so. In our zeal to promote adherence, we can often become so optimistic about the remarkable advances made in CF care that we may find it difficult to discuss the negative aspects of living with CF. We may minimize side effects, ignore (or more often, fail to ask about) symptoms we have no easy answer for, and we may be reluctant to discuss declining quality of life or eventual death. We are not unique among clinicians in this regard, but the dramatic advances made in $\mathrm{CF}$, as well as the increasingly available possibility of rescue by lung transplant, may make us particularly reluctant to utilize effective palliative care approaches which could increase quality of life. This difficulty can be amplified by our long-standing and close relationship with many patients and families, as we may avoid discussion of any distressing aspects of life with $\mathrm{CF}$ with those to whom we have become so close.

4. Shift in age at death. Changing demographics of death in CF have meant that skill in caring for the dying child or adolescent with CF has been lost; the death of a child or adolescent is now fortunately rare enough that our pediatric skills may be rusty. Inpatient clinical staff on adult wards are now caring for the majority of deaths from $\mathrm{CF}$, and we need to shift our focus to this clinical setting.

Factors Promoting the Integration of Palliative Care into CF care. 
1. A multi-disciplinary team approach to care. A team approach is a fact of life in the CF care model, and some members of the team may be more skilled at symptom assessment or communication about difficult topics. Yet all members of the team should become familiar with effective palliative care, and all members should learn to promote symptom assessment and treatment as well as open discussion of prognosis and treatment options. Under no circumstances should the burden of communication about prognosis or treatment options be left only to one team member.

2. A pattern of routine and preventive care. The routine nature of the $\mathrm{CF}$ care model gives ample time and opportunity to develop the rapport essential for shared decision-making. This is a luxury many of our colleagues in other clinical settings do not have, and we must make the most of it. Early discussion of advanced treatment options is a realistic goal in the CF model of care, although studies suggest we do not take advantage of the opportunity.

3. Widespread availability of expert palliative care teams. Palliative care services are increasingly available in clinical centers across North America, so that expertise in symptom management is far more accessible than in years past. However, anecdotal evidence and single center studies suggest that palliative care teams are invited onto the CF team only rarely and often at too late a stage to be helpful. This is another effect of the "either/or" thinking regarding palliative and curative therapy in $\mathrm{CF}$, but we can and must overcome this outdated approach.

Further Reading: 1. Koh JL, Harrison D, Palermo TM, et al. Assessment of acute and chronic pain symptoms in children with CF. Pediatr Pulmonol. 2005;40:330-5.

2. Palermo TM, Harrison D, Koh JL. Effect of diseaserelated pain on the health-related quality of life of children and adolescents with CF. Clin J Pain. 2006;22:532-7.

3. Sawicki GS, Sellers DE, Robinson WM. Self-reported physical and psychological symptom burden in adults with CF. J Pain Symptom Manage. 2008;35:372-80.

4. Ravilly S, Robinson W, Suresh S, et al. Chronic pain in cystic fibrosis. Pediatrics. 1996;98:741-7.
5. Lee A, Holdsworth M, Holland A, et al. The immediate effect of musculoskeletal physiotherapy techniques and massage on pain and ease of breathing in adults with CF. J Cyst Fibros. 2009;8:79-81.

6. Sermet-Gaudelus I, De Villartay P, de Dreuzy P, et al. Pain in children and adults with cystic fibrosis: A comparative study. J Pain Symptom Manage. 2009;38:281-90.

7. Stenekes SJ, Hughes A, Gregoire MC, et al. Frequency and self-management of pain, dyspnea, and cough in CF. J Pain Symptom Manage. 2009;38:837-48.

8. Walker LS, Smith CA, Garber J, et al. Testing a model of pain appraisal and coping in children with chronic abdominal pain. Health Psychol. 2005;24:36474.

9. Jones AM, Dodd ME, Webb AK, et al. Acute rib fracture pain in CF. Thorax. 2001;56:819.

10. Festini F, Ballarin S, Codamo T, et al. Prevalence of pain in adults with CF. J Cyst Fibros. 2004;3:51-7.

11. Hubbard PA, Broome ME, Antia LA. Pain, coping, and disability in adolescents and young adults with cystic fibrosis: A web-based study. Pediatr Nurs. 2005;31:82-6.

12. Robinson WM, Ravilly S, Berde C, et al. End-oflife care in cystic fibrosis. Pediatrics. 1997; 100:205-9.

13. Dellon EP, Leigh MW, Yankaskas JR, et al. Effects of lung transplantation on inpatient end of life care in CF. J Cyst Fibros. 2007;6:396-402.

14. Robinson WM. Palliative and end-of-life care in CF: what we know and what we need to know. Curr Opin Pulm Med. 2009 Jul 30 [Epub ahead of print].

15. Mitchell I, Nakielna E, Tullis E, et al. Cystic fibrosis: End stage care in Canada. Chest. 2000;118:80-4.

16. Dellon EP, Shores MD, Nelson KI, et al. Caregiver perspectives on discussions about the use of intensive treatments in CF. J Pain Symptom Manage. 2010;40:821-8.

17. Dellon EP, Shores MD, Nelson KI, et al. Family caregiver perspectives on symptoms and treatments for patients dying from complications of CF. J Pain Symptom Manage. 2010;40:829-37.

18. Dellon EW, Klick J, Robinson WM. Palliative care in children with CF. In: Wolfe J, Hinds P, Sourkes B, editors. Textbook of Interdisciplinary Pediatric Palliative Care. Philadelphia:Elsevier; 2011.

\title{
S17.4 \\ THE IMPACT OF TRANSPLANT ON ADVANCED CARE PLANNING AND END OF LIFE CARE OF THE CF PATIENT
}

\author{
Thomas G. Olbrych, M.D., FCCP ${ }^{1,2}$ \\ 1. Respiratory Institute, Cleveland Clinic, Cleveland, OH, USA; 2. Transplant Center, Cleveland Clinic, Cleveland, \\ $\mathrm{OH}, \mathrm{USA}$
}

Lung transplantation is indicated for patients with chronic end-stage lung disease who are failing maximal medical therapy, or for whom no effective medical therapy exists. The primary goal of lung transplantation is to provide a survival benefit, and several studies demon- strate that the survival of cystic fibrosis patients is improved following lung transplantation.

International Society of Heart and Lung Transplantation (ISHLT) guidelines for referral of a cystic fibrosis patient to a lung transplantation program include: 
1. FEV1 less than $30 \%$ of predicted, or rapid decline in FEV1, especially in young females.

2. An exacerbation of pulmonary disease requiring ICU care.

3. Increasing frequency of exacerbations requiring antibiotic therapy.

4. Recurrent and/or refractory pneumothorax.

5. Recurrent hemoptysis not controlled by embolization.

The ISHLT recommends listing cystic fibrosis patients for transplantation if they develop:

1. Oxygen-dependent respiratory failure.

2. Hypercapnia.

3. Pulmonary hypertension.

Lung transplantation for most patients as a palliative rather than curative treatment, and comparing the expected survival benefit from lung transplantation with improvement in quality of life remains a topic of considerable discussion in the cystic fibrosis and transplant communities.

When CF patients and their families are referred for Palliative Care, the primary goals are relief of symptoms and improvement in quality of life, whether or not such therapies may or may not shorten the duration of survival. Therefore, it is critically important to reconcile the concerns of patients, family, and caregivers during the terminal stages of cystic fibrosis lung disease with the requirements of that patient also listed for lung transplantation. Opiate analgesics may be exceedingly helpful in relieving pain associated with osteoporotic compression fractures, as well as providing relief of dyspnea in patients with any end-stage lung disease. However, osteoporosis will not improve following transplant, and it is important to keep in mind that regular preoperative use of these medications induces tolerance and leads to higher opiate doses needed to manage postoperative pain.
These higher doses may contribute to postoperative delirium and prolonged need for mechanical ventilation, delayed gastric emptying associated with nausea, emesis and aspiration pneumonia. While opiate pain medications are also effective in relieving dyspnea and anxiety associated with the end of life, inadvertent postoperative cessation of these medications may also be associated with delirium and withdrawal.

The use of noninvasive positive pressure ventilation (NIPPV, BiPAP) and/or endotracheal intubation and mechanical ventilation increases the Lung Allocation Score (LAS) and moves patients up the lung transplant waiting list. NIPPV is indicated for patients with chronic hypercarbic respiratory failure, and is particularly valuable as a bridge to transplantation in those cystic fibrosis patients already listed. Its use may provide valuable respiratory muscle rest, allowing patients to maintain ambulation and fitness critically important to successful transplant outcomes. Endotracheal intubation and positive pressure ventilation may keep the already listed patient alive until transplant, but the presence of an artificial airway that needs to be secured substantially limits activity and compromises overall conditioning. The presence of an endotracheal tube severely limits the patient's ability to communicate with family members and staff, critically important in assessing patient comfort needs at the end of life. The use of either of these modes of mechanical ventilatory support in the end-stage cystic fibrosis patient not listed for lung transplantation is of questionable value.

References: Orens JB, Estenne M, Arcasoy S, et al. International Guidelines for the Selection of Lung Transplant Candidates: 2006 Update - A Consensus Report From the Pulmonary Scientific Council of the International Society for Heart and Lung Transplantation. J Heart Lung Transplant. 2006:25:745-55.

\section{S18.1 \\ MOLECULAR BASIS FOR INTRINSIC PROINFLAMMATORY DEFECTS IN CF CELLS}

Harvey B. Pollard, M.D., Ph.D., Roopa Biswas, Ph.D., Meera Srivastava, Ph.D., Clifton L. Dalgard, Ph.D., Qingfeng Yang, M.D., Ph.D., Hung Caohuy, Ofer Eidelman, David M. Jacobowitz, Ph.D. and Greg P. Mueller

Anatomy, Physiology \& Genetics, USUHS School of Medicine, Bethesda, MD, USA

Cystic fibrosis $(\mathrm{CF})$ is an autosomal recessive disease, caused by mutations such as $[\Delta \mathrm{F} 508]$ in the CFTR gene, which prevent trafficking of the CFTR protein to the apical surface of lung epithelial cells. One important consequence is inability to activate the cAMP-activated chloride channel activity, which is intrinsic to CFTR. However, another consequence is the genesis of a profoundly hyper-proinflammatory phenotype in the airway, principally manifested by high levels of IL- 8 and certain other potent cytokines and chemokines. A frequent cause for debate is whether the pathophysiology of the CF airway is due to persistent infection, or due to intrinsic inflammation, or due to a mixture of both? Furthermore, because of operational defects in the innate immune system in the lung, it is possible that inflammation in the $\mathrm{CF}$ airway is potentiated by CFTR-dependent defects in immune cells, including neutrophils, macrophages and lymphocytes.

Is it Persistent Infection? One possibility for CF lung pathophysiology is that for an as yet unknown reason, the epithelial cells in the CF lung are hypersensitive to bacteria and other infectious agents. Consistently, cultured $\mathrm{CF}$ 
lung epithelial cells respond to bacteria and lipopolysaccharide (LPS) by expressing IL-8 at a greater rate than do lung epithelial cells from non-CF, control patients $(1,2)$. It has also been shown that Pseudomonas produces a toxin that suppresses whatever function is left in $[\Delta F 508] C F T R$ (3). Taken together, these pieces of information can be interpreted as consistent with enhanced sensitivity on the part of CF epithelial cells to infectious agents, possibly involving intrinsic activation of NFKB signaling.

Is it Intrinsic Inflammation? With respect to potential intrinsic connections between mutations in CFTR and production of IL-8 protein, the IL-8 mRNA in CF lung epithelial cells has been recently shown to be greatly stabilized relative to IL-8 mRNA in control cells (4). In this case, the mechanism involves low levels of the ARE-binding protein tristetraprolin (TTP), which regulates mRNA stability by interacting directly with ARE domains in the 3' untranslated region (3'UTR) of the IL8 mRNA. In addition, MAPKinases have direct control over IL-8 mRNA as well (5). We have also investigated the relationship between the presence of mutant CFTR and disease-specific microRNAs $(6,7)$. The microRNA miR155 was most elevated in CF cells, where it serves to activate signaling from PI3K to AKT. In turn, activated AKT drives downstream activation of MAPkinases, thereby stabilizing the IL-8 mRNA.

Is it Other Immune Cells in the CF lung? The proinflammatory phenotype of the CF lung has long been associated with objective defects in those neutrophils, macrophages and lymphocytes present in the airway. However, until recently, these defects have been ascribed to damage to otherwise normal immune cells, inflicted by the cytokine storm of epithelial cell dysfunction, infection, and obstruction. Macrophages, either resident in the CF lung, or studied in samples of peripheral circulation, have been found to express functional CFTR, which regulates phagosome acidification in macrophages (8). More recently, it has been shown that disease-causing mutations in CFTR can determine the functional responses of alveolar macrophages (9).

Likewise, neutrophils express CFTR, and apparently use it to translocate chloride into the phagolysosome, where it combines with $\mathrm{H}_{2} \mathrm{O}_{2}$ to produce bacteriocidal $\mathrm{HOCl}(10,11)$. $\mathrm{CF}$ neutrophils are found to be relatively inefficient at bacterial killing, and this defect may therefore also contribute to failure of the CF airway to clear the airway of bacteria. We have recently observed in CF neutrophils from blood that IL8 mRNA is massively elevated, and that genes associated with regulation of ER stress are particularly prominent (12).

Finally, in adaptive immunity experiments, lack of $\mathrm{Cftr}$ in CD3+ lymphocytes leads to an exaggerated $\mathrm{IgE}$ response when transferred into Cftr+ wildtype mice (13). The data clearly identify a Cftr deficiency in lymphocytes rather than the epithelial cells as the source of the dysfunctional signal.

The available data therefore seem to suggest "both," with an emphasis on CFTR-dependent proinflammato- ry signaling in lung epithelial cells. However, the available data also yield insight into why the CF airway cannot resolve infection. Disease-causing CFTR mutations affect the function of all the cells making up the lung's defensive armamentarium, leading to a perfect storm of functional incompetence. This insight may have relevance to the problem of drug development for $\mathrm{CF}$, because candidate therapeutics will have to rescue CFTR function in more than just the $\mathrm{CF}$ epithelial cell.

Acknowledgements: The authors acknowledge support from the NIH (RO1DK53051 [HBP]) and from the Cystic Fibrosis Foundation [HBP, RB].

References: 1. Venkatakrishnan A, Stacenko AA, King G, et al. Exaggerated activation of nuclear factorkappaB and altered iKappaB-beta processing in cystic fibrosis bronchial epithelial cells. Am J Respir Cell Mol Biol. 2000;23:396-403.

2. Muselet-Charlier C, Roque T, Boncoeur E, et al. Enhanced IL-1 beta-induced IL-8 production in CF lung epithelial cells is dependent of both mitogen-activated protein kinases and NF-kappaB signaling. Biochem Biophys Res Commun. 2007;357:402-7.

3. Bomberger JM, Ye S, Maceachran DP, et al. A $P$. aeruginosa toxin that hijacks the host ubiquitin proteolytic system. PLoS Pathog. 2011;7:e1001325.

4. Balakathiresan NS, Bhattacharyya S, Gutti U, et al. Tristetraprolin regulates IL-8 mRNA stability in cystic fibrosis lung epithelial cells. Am J Physiol Lung Cell Mol Physiol. 2009;296:L1012-8.

5. Bhattacharyya S, Gutti U, Mercado J, et al. MAPK signaling pathways regulate IL-8 mRNA stability and IL-8 protein expression in CF lung epithelial cells. Am J Physiol Lung Cell Mol Physiol. 2011;300:L81-7.

6. Biswas R, Bhattacharyya S, Balakathiresan N, et al. Mir155 regulates the proinflammatory phenotype in cystic fibrosis lung epithelial cells. Pediatr Pulmonol. 2010;S33:308.

7. Balakathiresan NS, Bhattacharyya S, Dalgard C, et al. Elevated miR-155 promotes inflammation in CF by driving hyper-expression of interleukin-8. J Biol Chem. 2011;286:11604-15.

8. Di A, Brown ME, Deriy LV, et al. CFTR regulates phagosome acidification in macrophages and alters bactericidal activity. Nat Cell Biol. 2006;8:933-44.

9. Deriy LV, Gomez EA, Zhang G, et al. Diseasecausing mutations in the CFTR determine the functional responses of alveolar macrophages. J. Biol Chem. 2009;284:35926-38.

10. Painter RG, Valentine VG, Lanson NA Jr, et al. CFTR expression in human neutrophils and the phagolysosomal chlorination defect in CF. Biochemistry. 2006;5:10260-9.

11. Painter RG, Marrero L, Lombard GA, et al. CFTR-mediated halide transport in phagosomes of human neutrophils. J Leukoc Biol. 2010;87:933-42. 
12. Dalgard CL, Bhattacharyya S, Biswas R, et al. Genome wide transcriptome profiling of CF blood neutrophils reveals inflammation, gender and CFTR-dependent expression differences. Pediatr Pulmonol 2010;S33:235.
13. Mueller C, Braag SA, Keeler A, et al. Lack of cystic fibrosis transmembrane conductance regulator in CD3+ lymphocytes leads to aberrant cytokine secretion and hyper-inflammatory adaptive immune responses. Am J Respir Cell Mol Biol. 2010;44:922-9.

\title{
S18.2 \\ CONTRIBUTION OF THE INNATE IMMUNE SYSTEM AND TOLL-LIKE RECEPTOR SIGNALLING TO LUNG-DAMAGING INFLAMMATION IN CYSTIC FIBROSIS
}

\author{
Stuart E. Turvey, M.D., Ph.D. and Christoph J. Blohmke \\ Dept. of Pediatrics, Univ. of British Columbia, Child \& Family Research Institute, Vancouver, BC, Canada
}

Lung disease, the major cause of death in cystic fibrosis $(\mathrm{CF})$ is caused by chronic infection and inflammation. Current therapies for CF address airway infection with antibiotics, and airway obstruction using clearance techniques combined with mucolytics. Safe and clinically-acceptable therapies to target airway inflammation are likely to augment current treatments and improve the clinical outcome in CF (1). Clinical trials with oral corticosteroids, high-dose ibuprofen and azithromycin have demonstrated that anti-inflammatory therapy is beneficial for patients with $\mathrm{CF}$, improving important clinical outcomes such as lung function and body weight. Clinical experience with these relatively non-specific antiinflammatory medications provides "proof of concept" evidence that targeting inflammation can be beneficial in CF. Our challenge is now to identify new, specific antiinflammatory targets.

Modulating the function of the innate immune system is a particularly attractive treatment approach, since activation of the innate immune system is central to the inflammatory response occurring in the CF lung (2). Innate immunity relies upon a series of germline-encoded receptors, including Toll-like receptors (TLRs), to sense infectious organisms and to trigger an acute inflammatory response. Work by our group and others suggests that modulation of TLR function, and particularly TLR5 inhibition, has therapeutic potential to improve the inflammatory manifestations of CF $(3,4)$. Emerging evidence indicates that the TLR5-flagellin interaction plays a central role in driving the inflammatory response triggered by $P$. aeruginosa and inhibition of TLR5 normalizes the inflammatory response generated by $\mathrm{CF}$ airway epithelial cells following exposure to $P$. aeruginosa (4).

A powerful translational research approach for identifying and validating new therapeutic targets is through the identification of genetic variants that modify CF clinical phenotypes. The TLR5 gene harbours a polymorphism-TLR5 c. $1174 \mathrm{C}>\mathrm{T}$ (rs5744168) — that encodes a premature stop codon. Since allele T has been shown by our group and others (5) to be associated with significantly impaired flagellin responsiveness, we predicted that $\mathrm{CF}$ patients carrying the $\mathrm{T}$ allele would have improved clinical outcomes due to the endogenous antiinflammatory effects of this TLR5 variant. In a large representative CF cohort, adults with CF carrying the TLR5 premature stop codon (CT or TT genotype) had improved nutritional status, as measured by zBMI, compared with $\mathrm{CF}$ patients homozygous for the common fully-functional allele (CC genotype) (6). Nevertheless, our results must be interpreted with some caution given that the association between the TLR5 c.1174C>T SNP and $z B M I$ in adult $C F$ patients was modest $(\mathrm{P}=0.044)$ and the modifying impact of TLR5 will only be firmly established through replication studies in other CF cohorts.

A major challenge confronting our CF research community and our efforts to successfully control pulmonary inflammation is to understand the molecular mechanism(s) mediating inflammatory immune responses in the CF airway. Despite an increasing appreciation of how $\mathrm{CF}$ pathogens interact with the airway epithelium, it remains unclear whether inflammation in CF is inherently triggered by loss-of-function CFTR mutations alone or due to the failure of the innate immune system to control infection of the airways. Many studies support the concept that excess inflammation is a fundamental component of CF. For example, even in the absence of culturable infections increased pro-inflammatory molecules are found in bronchoalveolar lavage (BAL) of CF patients (7) and immortalized fetal CF cells showed enhanced activation of pro-inflammatory pathways (8). While the lack of CFTR channel function (9) and endoplasmic reticulum (ER) stress-induced inflammatory responses (10) have been suggested as potential causes, the mechanisms underlying the exaggerated inflammatory phenotype in $\mathrm{CF}$ are not clear. In some studies p38 MAPK and ERK signalling is dysregulated in CF with potentially detrimental consequences including increased activation of pro-inflammatory transcription

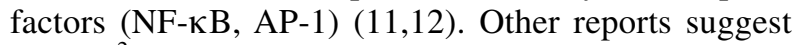
that $\mathrm{Ca}^{2+}$ signalling or an imbalance in the oxidative stress response in $\mathrm{CF}$ potentially mediates the enhanced activation of pro-inflammatory signalling $(10,13)$.

Nevertheless, the evidence supporting a hyperinflammatory phenotype in $\mathrm{CF}$ is far from unanimous with 
some studies indicating that airway inflammation in CF only follows respiratory infection (14-17). Similarly, in pigs with mutated CFTR genes no inflammation is present at birth; but very early in life, CF piglets develop signs of CF lung disease including infection, airway inflammation and mucus accumulation suggesting a defect in host-defence mechanisms (18).

The controversy of whether lung inflammation in $\mathrm{CF}$ can occur in the absence of infection, while important for understanding the basic pathophysiology of $\mathrm{CF}$, is less relevant for the treatment of $\mathrm{CF}$ patients. Airway infection and inflammation are virtually universal in $\mathrm{CF}$ patients (regardless of the order in which they develop) and reducing inflammation is likely to improve CF lung disease. We must now identify the molecular pathways that can be targeted to safely and effectively reduce lungdamaging inflammation in CF.

References: 1. Chmiel JF, Konstan MW. Inflammation and anti-inflammatory therapies for cystic fibrosis. Clin Chest Med. 2007;28:331-46.

2. Doring G, Gulbins E. Cystic fibrosis and innate immunity: How chloride channel mutations provoke lung disease. Cell Microbiol. 2009;11:208-16.

3. Greene CM, Branagan P, McElvaney NG. Toll-like receptors as therapeutic targets in CF. Expert Opin Ther Targets. 2008;12:1481-95.

4. Blohmke CJ, Victor RE, Hirschfeld AF, et al. Innate immunity mediated by TLR5 as a novel antiinflammatory target for CF lung disease. J Immunol. 2008;180:7764-73.

5. Hawn TR, Verbon A, Lettinga KD, et al. A common dominant TLR5 stop codon polymorphism abolishes flagellin signaling and is associated with susceptibility to legionnaires' disease. J Exp Med. 2003;198:1563-72.

6. Blohmke CJ, Park J, Hirschfeld AF, et al. TLR5 as an anti-inflammatory target and modifier gene in CF. J Immunol. 2010;185:7731-8.

7. Khan TZ, Wagener JS, Bost T, et al. Early pulmonary inflammation in infants with cystic fibrosis. Am J Respir Crit Care Med. 1995;151:1075-82.
8. Verhaeghe C, Remouchamps C, Hennuy B, et al. Role of IKK and ERK pathways in intrinsic inflammation of CF airways. Biochem Pharmacol. 2007;73:1982-94.

9. Perez A, Issler AC, Cotton CU, et al. CFTR inhibition mimics the cystic fibrosis inflammatory profile. Am J Physiol Lung Cell Mol Physiol. 2007;292:L383-95.

10. Rottner M, Freyssinet JM, Martinez MC. Mechanisms of the noxious inflammatory cycle in CF. Respir Res. 2009;10:23.

11. Berube J, Roussel L, Nattagh L, et al. Loss of cystic fibrosis transmembrane conductance regulator function enhances activation of p38 and ERK MAPKs, increasing interleukin-6 synthesis in airway epithelial cells exposed to $P$. aeruginosa. J Biol Chem. 2010;285:22299-307.

12. Bhattacharyya S, Gutti U, Mercado J, et al. MAPK signaling pathways regulate IL-8 mRNA stability and IL-8 protein expression in CF lung epithelial cells lines. Am J Physiol Lung Cell Mol Physiol. 2011;300:L81-7.

13. Tabary O, Boncoeur E, de Martin R, et al. Calcium-dependent regulation of NF-(kappa)B activation in CF airway epithelial cells. Cell Signal. 2006;18:652-60.

14. Armstrong DS, Grimwood K, Carlin JB, et al. Lower airway inflammation in infants and young children with CF. Am J Respir Crit Care Med. 1997;156:1197-204.

15. Armstrong DS, Grimwood K, Carzino R, Carlin JB, Olinsky A, Phelan PD. Lower respiratory infection and inflammation in infants with newly diagnosed cystic fibrosis. BMJ. 1995;310:1571-2.

16. Machen TE. Innate immune response in $\mathrm{CF}$ airway epithelia: hyperinflammatory? Am J Physiol Cell Physiol. 2006;291:C218-30.

17. Armstrong DS, Hook SM, Jamsen KM, et al. Lower airway inflammation in infants with $\mathrm{CF}$ detected by newborn screening. Pediatr Pulmonol. 2005;40:500-10.

18. Stoltz DA, Meyerholz DK, Pezzulo AA, et al. Cystic fibrosis pigs develop lung disease and exhibit defective bacterial eradication at birth. Sci Transl Med. 2010;2:29ra31.

\title{
S18.3 \\ CFTR AND PHAGOCYTIC HOST DEFENSE
}

\author{
Guoshun Wang, DVM, Ph.D. \\ Microbiology, Immunology \& Parasitology, Louisiana State Univ. Health Sciences Ctr., New Orleans, LA, USA
}

Innate immunity in CF pathogenesis. Cystic fibrosis $(\mathrm{CF})$ is a multi-system and multi-organ disease. Because CF lung disease claims the majority of morbidity and mortality clinically, current research efforts chiefly focus on the lung and airway epithelia. However, if CF lung disease is solely an epithelial problem, then, lung transplantation by giving the patients new lungs would solve all the issues. Our retrospective clinical data analyses indicate that $\mathrm{CF}$ lung-transplant patients contract Pseudomonas lung infection much earlier and more frequently than the non-CF lung transplants (1). This observation cannot satisfactorily be explained by the theory of bacterial predisposition in CF sinus and upper airways. 
CF and early microbe-host interactions. The most devastating anomaly of CF occurs in the lung characterized by chronic bacterial infection, abnormal airway inflammation, extensive neutrophil infiltration and small airway obstruction (2-3). CF lung infection has a unique pathogen profile which is distinct from other lung infections. Pseudomonas aeruginosa, Staphylococcus aureus, Haemophilus influenzae, Stenotrophomonas maltophilia, Achromobacter xylosoxidans, Burkholderia cepacia are the most prevalent, among which $P$. aeruginosa predominates (4-6). Strikingly, all the CF organisms except S. aureus are opportunistic pathogens, which do not cause infections in normal hosts but in neutropenic patients (6). It is not fully understood why CF patients are particularly susceptible to these organisms and how the organisms manage to escape the host defense at the early infection stages when there is little antibiotic selection and no biofilm formation. It is the early microbehost interactions and the early subversion of the host defense that leads to pathogen colonization and subsequently persistent infection in CF lungs.

Polymorphonuclear neutrophils and anti-bacterial defense. Neutrophils constitute $50-60 \%$ of the circulating leukocyte pool in humans and are the major cell type to combat extracellular bacterial infections (7). Neutrophils ingest bacteria by a process known as phagocytosis (8). After the bacteria are contained in the membrane-bounded phagosomes, neutrophils produce toxic oxidants including hydrogen peroxide $\left(\mathrm{H}_{2} \mathrm{O}_{2}\right)$ and hypochlorous acid $(\mathrm{HOCl})$ to achieve an efficient killing (9-10). $\mathrm{HOCl}$, the active component in common laundry bleach, is synthesized by myeloperoxidase (MPO) predominantly presented in neutrophils. This MPO-catalyzed chemical reaction, $\mathrm{H}_{2} \mathrm{O}_{2}+\mathrm{Cl}^{-}+\mathrm{H}^{+} \rightarrow \mathrm{HOCl}+\mathrm{H}_{2} \mathrm{O}$, requires $\mathrm{Cl}^{-}$as a substrate. Thus, the availability of this anion to neutrophil phagosomes is a rate-limiting factor, which affects the production of $\mathrm{HOCl}$. Such a defect in phagocytic innate immunity may preferentially allow certain bacterial strains to evade the compromised host defense.

Neutrophils express CFTR and mobilize this chloride channel to their phagosomes. Human peripheral blood neutrophils express CFTR as noted by immunofluorescent staining. When neutrophils phagocytose bacteria, CFTR is recruited to the phagosomes (11). PLB985 cells are premyelocytic cell line. We have established a permanent cell line expressing EGFP-wt-CFTR. After induction with 1.25\% DMSO, the cells differentiate into mature neutrophils which internalize opsonized 3-micron Latex beads. CFTR is rapidly mobilized to the nascent phagosomal membrane and persists through phagosomal maturation. Therefore, CFTR recruitment to phagosomal membrane is an early event, which reflects the importance of the CFTR channel in neutrophil functions.
CF neutrophils have chloride transport and bacterial killing defects. To directly measure chloride levels in neutrophil phagosomes, we conjugated chloride probe to zymosan particles. After phagocytosis by normal or $\mathrm{CF}$ neutrophils, fluorescence of the probe was quantitatively measured and the dynamics of the chloride level in phagosomes in response to extracellular chloride level changes were recorded. The data demonstrated that $\mathrm{CF}$ neutrophils are deficient in transporting chloride to the organelle (12). Furthermore, CF neutrophil microbicidal function is impaired, especially in a low chloride environment (13).

Summary. Mounting evidence indicates that CFTR plays an important role in phagocytic host defense, which may contribute to $\mathrm{CF}$ lung pathogenesis.

References: 1. Bonvillain RW, Valentine VG, Lombard G, et al. Post-operative infections in cystic fibrosis and non-cystic fibrosis patients after lung transplantation. J Heart Lung Transplant. 2007;26:890-7.

2. Welsh MJ, Ramsey BW, Accurso F, et al. Cystic Fibrosis. In: Scriver CR, editor. Metabolic and Molecular Basis of Interited Disease. New York: McGraw-Hill, 2001. p. 5121-88.

3. Davis PB, Drumm M, Konstan MW. Cystic fibrosis. Am J Respir Crit Care Med. 1996;154:1229-56.

4. Sadikot RT, Blackwell TS, Christman JW, et al. Pathogen-host interactions in $P$. aeruginosa pneumonia. Am J Respir Crit Care Med. 2005;171:1209-23.

5. Cystic Fibrosis Foundation Patient Registry: 2009 Annual Data Report, p. 15. Available from: http://www.cff.org/research/ClinicalResearch/PatientRegistryReport/.

6. Gibson RL, Burns JL, Ramsey BW. Pathophysiology and management of pulmonary infections in CF. Am J Respir Crit Care Med. 2003;168:918-51.

7. Nauseef WM. How human neutrophils kill and degrade microbes: an integrated view. Immunol Rev. 2007;219:88-102.

8. Flannagan RS, Cosio G, Grinstein S. Antimicrobial mechanisms of phagocytes and bacterial evasion strategies. Nat Rev Microbiol. 2009;7:355-66.

9. Hampton MB, Kettle AJ, Winterbourn CC. Inside the neutrophil phagosome: oxidants, myeloperoxidase, and bacterial killing. Blood. 1998;92:3007-17.

10. Klebanoff SJ. Myeloperoxidase: friend and foe. J Leukoc Biol. 2005;77:598-625.

11. Painter RG, Valentine VG, Lanson NA Jr, et al. CFTR expression in human neutrophils and the phagolysosomal chlorination defect in CF. Biochemistry. 2006;45:10260-9.

12. Painter RG, Marrero L, Lombard GA, et al. CFTR-mediated halide transport in phagosomes of human neutrophils. J Leukoc Biol. 2010;87:933-42.

13. Painter RG, Bonvillain RW, Valentine VG, et al. The role of chloride anion and CFTR in killing of $P$. aeruginosa by normal and CF neutrophils. J Leukoc Biol. 2008;83:1345-53. 


\title{
S18.4 \\ PSEUDOMONAS AERUGINOSA REDUCES THE VIRAL IMMUNE RESPONSE IN THE CF AIRWAY
}

\author{
Jennifer Bomberger, Ph.D. ${ }^{1}$, Roxanna Barnaby ${ }^{2}$, Naveen Bangia ${ }^{3}$ and Bruce Stanton ${ }^{2}$ \\ 1. Microbiology \& Molecular Genetics, Univ. of Pittsburgh, Pittsburgh, PA, USA; 2. Microbiology \& Immunology, \\ Dartmouth Medical School, Hanover, NH, USA; 3. Immunology, Roswell Park Cancer Institute, Buffalo, NY, USA
}

Background. Pseudomonas aeruginosa is an opportunistic pathogen and a leading cause of nosocomial infections, especially in a setting of epithelial cell injury and in immunocompromised patients, including cystic fibrosis (CF) patients (1). Mutations in CFTR reduce or eliminate chloride secretion in the lung and thereby reduce the volume of airway surface liquid, which in turn suppresses mucociliary clearance of $P$. aeruginosa and other pathogens trapped in the mucus $(2,3)$. Accordingly, individuals with $\mathrm{CF}$ are susceptible to chronic infections, primarily by the opportunistic pathogen $P$. aeruginosa. Over time the CF lung evolves into a highly inflamed and purulent environment that is the proximate cause of morbidity and mortality in these patients. By late adolescence, $80 \%$ of CF patients are chronically infected with $P$. aeruginosa, the dominant pathogen in CF airways (46). As is the case with CF, patients with COPD and bronchiectasis are particularly susceptible to bacterial infection, including colonization by $P$. aeruginosa, which is particularly difficult to eradicate due to the high basal levels of antibiotic resistance and the ability of $P$. aeruginosa to form antimicrobial resistant biofilms (3,7-11).

In the CF lung, the virulence factors produced by this microbe and the chronic immune response mounted by the host in a fruitless attempt to clear these infections combine to cause extensive damage to the lung tissue, and frequently, respiratory failure and death of the patient (4-6). Patients with chronic P. aeruginosa colonization often acquire respiratory virus infections, which may trigger acute exacerbations. Synergism between bacteria and viruses in inducing infection in the airway is welldocumented $(12,13)$. CF patients show reduced ability to clear $P$. aeruginosa acquired during respiratory viral infections compared to healthy controls, often requiring anti-pseudomonal treatment (14). Furthermore, $85 \%$ of new pseudomonal colonization of CF patients followed a viral upper respiratory tract infection within 3 weeks (15). Most studies to date characterize an infection where the virus predisposes patients for a secondary bacterial infection, but scientists and clinicians now recognize that the synergism is not unidirectional $(16,17)$. In fact, intrapulmonary influenza titers are also elevated in mice with chronic $P$. aeruginosa infection compared to $P$. aeruginosa free mice (18). And although the prevalence of viral disease is similar in CF and healthy controls, CF patients with viral lung infections have prolonged and exacerbated symptoms and longer hospitalizations $(12,19)$. The abundance of literature documenting respiratory viral and bacterial, often P. aeruginosa in CF, dual infections have not established a mechanism of how these polymicrobial infections arise or how they are maintained during the course of the ensuing respiratory disease. Our long-term goal is to define a mechanism for the prolonged and exacerbated viral infections observed in the clinic in CF patients chronically colonized by P. aeruginosa.

Results. We previously reported that a secreted toxin from $P$. aeruginosa, Cif (PA2934), reduces CFTR-mediated chloride secretion by human airway epithelial cells by enhancing the amount of ubiquitinated CFTR and its degradation via the lysosomal pathway (20-22). The aim of the current study was to determine if Cif regulates other $\mathrm{ABC}$ transporters, specifically the transporter associated with antigen processing (TAP) to alter viral antigen presentation. Patients with TAP deficiency, like CF patients, have recurrent bacterial infections, nasal polyps, and chronic purulent rhinitis in the first 6 years of life, and chronic bacterial infections, bacterial pneumonia, and bronchiectasis later in life. Thus, we hypothesized that Cif facilitates the degradation of the TAP complex, resulting in reduced viral antigen presentation via the major histocompatibility complex (MHC) class I molecules and therefore, diminishes the immune response to viral pathogens. P. aeruginosa toxin Cif reduced TAP1, but not TAP2, protein abundance in polarized human airway epithelial cells (CFBE41o- cells) in a time-dependent manner. The Cif-mediated reduction in TAP1 was more dramatic in CF airway cells (CFBE41o-), as compared to WT-CFTR complemented CFBE41o- cells. Cif inhibited ubiquitin specific protease 10 (USP10), a deubiquitinating enzyme, resulting in increased poly-ubiquitination and proteasomal degradation of TAP1. The inhibition of USP10 activity by Cif was more profound in CF cells (CFBE41o-), as compared to WT-CFTR complemented CFBE41o- cells. USP10 deubiquitinating activity is regulated by protein-protein interactions with G3BP1 (Ras-GAP SH3 domain binding protein), where interaction of the N-terminus of USP10 with G3BP1 inactivates USP10 DUB activity (23). Cif does not alter the protein abundance of USP10, but does enhance the interaction between G3BP1 and USP10 to inhibit USP10 DUB activity. In CF airway cells, G3BP1 protein abundance is increased, leading to a reduced basal level of USP10 activity and thus, greater susceptibility 
to the Cif toxin. Functionally, the Cif-mediated decrease in TAP1 expression reduced peptide uptake into the endoplasmic reticulum and decreased $\mathrm{MHC}$ class I molecule abundance at the plasma membrane, effects that were enhanced in CF cells compared to WT-CFTR complemented CF cells. Furthermore, Cif decreased MHC class I presentation of viral antigen and eliminated CD8 $\mathrm{T}$ cell recognition of influenza Ainfected cells.

Summary. We propose that $P$. aeruginosa, by secreting the Cif toxin, inhibits the ability of CD8 T cells to recognize and eliminate viral infections, severely compromising the immune defenses in the lungs of $\mathrm{CF}$ patients. This is the first bacterial toxin that inhibits the viral immune response of the host, potentially illuminating a mechanism for the clinical observation that viral-bacterial co-infections dramatically increase the morbidity and mortality of CF patients chronically colonized by $P$. aeruginosa. This study also identifies a new therapeutic target for preventing co-infections in the $\mathrm{CF}$ airway.

References: 1. Govan JR, Nelson JW. Microbiology of lung infection in cystic fibrosis. Br Med Bull. 1992;48:912-30.

2. Davis PB. Cystic fibrosis since 1938. Am J Respir Crit Care Med. 2006;173:475-82.

3. Boucher RC. New concepts of the pathogenesis of CF lung disease. Eur Respir J. 2004;23:146-58.

4. Costerton JW. Cystic fibrosis pathogenesis and the role of biofilms in persistent infection. Trends Microbiol. 2001;9:50-2.

5. Lyczak JB, Cannon CL, Pier GB. Lung infections associated with CF. Clin Microbiol Rev. 2002;15:194-222.

6. Heijerman $\mathrm{H}$. Infection and inflammation in CF: a short review. J Cyst Fibros. 2005;4(Suppl 2):3-5.

7. Mah TF, O'Toole GA. Mechanisms of biofilm resistance to antimicrobial agents. Trends Microbiol. 2001;9:34-9.

8. Driscoll JA, Brody SL, Kollef MH. The epidemiology, pathogenesis and treatment of $P$. aeruginosa infections. Drugs. 2007;67:351-68.

9. Moreau-Marquis S, Bomberger JM, Anderson GG, et al. The DeltaF508-CFTR mutation results in increased biofilm formation by $P$. aeruginosa by increasing iron availability. Am J Physiol Lung Cell Mol Physiol. 2008;295:L25-37.
10. Singh PK, Schaefer AL, Parsek MR, et al. Quorum-sensing signals indicate that $\mathrm{CF}$ lungs are infected with bacterial biofilms. Nature. 2000;407:762-4.

11. Worlitzsch D, Tarran R, Ulrich M, et al. Effects of reduced mucus oxygen concentration in airway Pseudomonas infections of CF patients. J Clin Invest. 2002;109:317-25.

12. Van Ewijk BE, Wolfs TF, Aerts PC, et al. RSV mediates $P$. aeruginosa binding to cystic fibrosis and normal epithelial cells. Pediatr Res. 2007;61:398-403.

13. Beadling C, Slifka MK. How do viral infections predispose patients to bacterial infections? Curr Opin Infect Dis. 2004;17:185-91.

14. Van Ewijk BE, Wolfs TF, Fleer A, et al. High $P$. aeruginosa acquisition rate in CF. Thorax. 2006;61:641-2.

15. Collinson J, Nicholson KG, Cancio E, et al. Effects of upper respiratory tract infections in patients with CF. Thorax. 1996;51:1115-22.

16. Tashiro M, Ciborowski P, Reinacher M, et al. Synergistic role of staphylococcal proteases in the induction of influenza virus pathogenicity. Virology. 1987;157:421-30.

17. Tashiro M, Ciborowski P, Klenk HD, et al. Role of Staphylococcus protease in the development of influenza pneumonia. Nature. 1987;325:536-7.

18. Seki M, Higashiyama Y, Tomono K, et al. Acute infection with influenza virus enhances susceptibility to fatal pneumonia following Streptococcus pneumoniae infection in mice with chronic pulmonary colonization with $P$. aeruginosa. Clin Exp Immunol. 2004;137:35-40.

19. Wat D, Doull I. Respiratory virus infections in cystic fibrosis. Paediatr Respir Rev. 2003;4:172-7.

20. Swiatecka-Urban A, Moreau-Marquis S, Maceachran DP, et al. $P$. aeruginosa inhibits endocytic recycling of CFTR in polarized human airway epithelial cells. Am J Physiol Cell Physiol. 2006;290:C862-72.

21. Bomberger JM, Ye S, Maceachran DP, et al. A $P$. aeruginosa toxin that hijacks the host ubiquitin proteolytic system. PLoS Pathog. 2011;7:e1001325.

22. Bomberger JM, Maceachran DP, Coutermarsh BA, et al. Long-distance delivery of bacterial virulence factors by $P$. aeruginosa outer membrane vesicles. PLoS Pathog. 2009;5:e1000382.

23. Soncini C, Berdo I, Draetta G. Ras-GAP SH3 domain binding protein (G3BP) is a modulator of USP10, a novel human ubiquitin specific protease. Oncogene. 2001;20:3869-79.

\title{
S18.5 \\ LACK OF FUNCTIONAL CFTR PRIMES AIRWAY EPITHELIAL CELLS TO HYPER RESPONSIVENESS TO INJURIOUS STIMULI
}

\author{
Trevor Beaudoin, B.Sc, M.Sc, Lucie Roussel, Ph.D., Julie Bérubé, M.Sc. and Simon Rousseau, Ph.D. \\ Department of Medicine, McGill University, Montreal, QC, Canada
}

Defective cystic fibrosis transmembrane conductance regulator (CFTR) function is responsible for cystic fibro- sis (CF) lung disease, the most life-threatening complication of CF. Lung function declines and fails as a con- 
sequence of airway inflammation, chronic bacterial infections and progressive tissue destruction. Given the critical role of lung inflammation in the pathogenesis of CF lung disease, a better understanding of the relationship between infections and inflammation is critical for the development of novel therapies targeting inflammation. An important question within this relationship has been whether inflammation or infection comes first.

A limited and remarkably consistent collection of bacteria colonizes and infects the CF lung in an age dependent sequence (1), suggesting an intrinsic defect that provides a niche for specific infections. Pulmonary infections start early in children with CF, with Staphylococcus aureus common in young infants (1). Other organisms, such as Haemophilus influenzae and Pseudomonas aeruginosa, are believed to become more prevalent in older children and adults. Up to $80 \%$ of the patients are eventually infected with $P$. aeruginosa (2). $P$. aeruginosa can be found as free-swimming, planktonic bacteria or as micro-colonies encased in an exopolymeric matrix, also known as biofilms. It has been suggested that chronic infections of $P$. aeruginosa in CF represent a prototypical biofilm infection, with the formation of large cellular aggregates of the bacteria and distinct production of molecular patterns (3).

The airway epithelium is the first line of defense against foreign particles and pathogens; it not only forms a physical barrier, but also plays an important role in the detection of pathogens and the initiation of inflammatory responses. This is accomplished via an intricate network of signaling pathways, both within and between cells. Activation of this network occurs via pattern-recognition receptors (PRRs), so-called because they recognize molecular patterns from bacteria, viruses and certain selfderived molecules. The two best-characterized families of PRRs are the Toll-Like Receptors (TLRs) and the NODlike Receptors (NLRs). A convergence point of host defense mechanisms against bacterial pathogens activated by many PRRs is the $\mathrm{p} 38 \alpha$ Mitogen-Activated Protein Kinase (MAPK) (4). Interestingly, nematodes deficient for pmk-1, a p38 MAPK ortholog, are susceptible to bacterial killing by $P$. aeruginosa (5). This highlights the ancient role of p38 $\alpha$ MAPK in regulating host defense against this pathogen and its usefulness as a marker of engagement of host innate immune responses to $P$. aeruginosa.

In human airway epithelial cells (AECs), activation of $\mathrm{p} 38 \alpha$ MAPK has been coupled to TLR5 in response to $P$. aeruginosa (6). Interestingly TLR5 has been reported to be an anti-inflammatory target and modifier gene in CF $(7,8)$. However, it is unclear whether $P$. aeruginosa grown as a biofilm activates AECs in the same way as planktonic $P$. aeruginosa. Comparing the activation of p38 $\alpha$ MAPK in response to planktonic or biofilm derived $P$. aeruginosa materials, we discovered that planktonic $P$. aeruginosa activates p38 $\alpha$ MAPK in a TLR-dependent fashion more strongly than biofilm $P$. aeruginosa, which acted in a TLR-independent fashion. This highlights the complex relationship between $P$. aeruginosa and the airway epithelium in $\mathrm{CF}$; airway epithelial cells distinguish different bacterial forms of the same clinical isolate using distinct pathways for their detection.

Moreover, in CF a "hyper-inflammatory" phenotype has been described, resulting in increased synthesis of pro-inflammatory cytokines (9-12). Furthermore, we recently described that CFTRdelF508 AECs are hypersensitive to bacterial stimuli, resulting in increased MAPK activation and IL-6/IL8 synthesis $(13,14)$. We also found that CFTRdelF508 AECs are more sensitive to reactive oxygen species (14). A pro-oxidative shift is associated with enhanced intracellular signaling (15), which could explain increased MAPK activation and pro-inflammatory cytokine secretion. Interestingly, this hyper-responsiveness holds true in response to both planktonic and biofilm derived material from $P$. aeruginosa.

These results suggest that p38 $\alpha$ MAPK reaches a functional threshold of activation required to engage host defense mechanisms earlier in CF. This lower threshold of activation means that AEC from $\mathrm{CF}$ patients may engage pro-inflammatory responses more frequently than their non-CF counterpart, resulting over time in increased tissue damage.

References: 1. Li Z, Kosorok MR, Farrell PM, et al. Longitudinal development of mucoid $P$. aeruginosa infection and lung disease progression in children with cystic fibrosis. JAMA 2005;293:581-8.

2. Lipuma JJ. The changing microbial epidemiology in cystic fibrosis. Clin Microbiol Rev. 2010;23:299-323.

3. Singh PK, Schaefer AL, Parsek MR, et al. Quorumsensing signals indicate that $\mathrm{CF}$ lungs are infected with bacterial biofilms. Nature. 2000;407:762-4.

4. Rousseau S. p38 alpha MAP kinase. UCSD-Nature Molecule Pages. Published online: 15 Mar 2011 doi:10.1038/mp.a001717.01. Available from: http://www.signaling-gateway.org/molecule/query?afcsid=A001717.

5. Kim DH, Feinbaum R, Alloing G, et al. A conserved p38 MAP kinase pathway in Caenorhabditis elegans innate immunity. Science. 2002;297:623-6.

6. Zhang Z, Reenstra W, Weiner DJ, et al. The p38 mitogen-activated protein kinase signaling pathway is coupled to Toll-like receptor 5 to mediate gene regulation in response to $P$. aeruginosa infection in human airway epithelial cells. Infect Immun. 2007;75:5985-92.

7. Blohmke CJ, Park J, Hirschfeld AF, et al. TLR5 as an anti-inflammatory target and modifier gene in CF. J Immunol. 2010;185:7731-8.

8. Blohmke CJ, Victor RE, Hirschfeld AF, et al. Innate immunity mediated by TLR5 as a novel antiinflammatory target for CF lung disease. J Immunol. 2008;180:7764-73. 
9. Stecenko AA, King G, Torii K, et al. Dysregulated cytokine production in human cystic fibrosis bronchial epithelial cells. Inflammation. 2001;25:145-55.

10. Kube D, Sontich U, Fletcher D, et al. Proinflammatory cytokine responses to $P$. aeruginosa infection in human airway epithelial cell lines. Am J Physiol Lung Cell Mol Physiol. 2001;280:L493-502.

11. Becker MN, Sauer MS, Muhlebach MS, et al. Cytokine secretion by CF airway epithelial cells. Am J Respir Crit Care Med. 2004;169:645-53.

12. Bonfield TL, Konstan MW, Berger M. Altered respiratory epithelial cell cytokine production in CF. J Allergy Clin Immunol. 1999;104:72-8.
13. Roussel L, Martel G, Berube J, et al. P. aeruginosa drives CXCL8 synthesis via redundant toll-like receptors and NADPH oxidase in CFTR $\varnothing F 508$ airway epithelial cells. J Cyst Fibros. 2011;10:107-13.

14. Berube J, Roussel L, Nattagh L, et al. Loss of cystic fibrosis transmembrane conductance regulator function enhances activation of p38 and ERK MAPKs, increasing interleukin-6 synthesis in airway epithelial cells exposed to $P$. aeruginosa. J Biol Chem. 2010;285:22299-307.

15. Allen RG, Tresini M. Oxidative stress and gene regulation. Free Radic Biol Med. 2000;28:463-99.

\title{
S19.1 \\ THE GENETIC BASIS OF MECONIUM ILEUS
}

\author{
Lisa J. Strug, Ph.D. ${ }^{1,2}$ and Gene Modifiers for the International Consortium of $\mathrm{CF}^{3,4}$ \\ 1. Child Health Evaluative Sciences, The Hospital for Sick Children, Toronto, ON, Canada; 2. Dalla Lana School of \\ Public Health, Univ. of Toronto, Toronto, ON, Canada; 3. Univ. Pierre et Marie Curie-Paris 6, Paris, France; 4. \\ Johns Hopkins Univ., UNC \& Case Western Reserve Univ., Baltimore, Chapel Hill \& Cleveland, OH, USA
}

Meconium ileus (MI) is a severe intestinal obstruction which presents at birth. It is the earliest clinical manifestation of cystic fibrosis $(\mathrm{CF})$ and occurs in $\sim 15 \%$ of $\mathrm{CF}$ patients. It occurs with equal frequency in males and females, and the proportion of observed variation in $\mathrm{MI}$ that can be attributed to inherited genetic factors, i.e. the heritability of MI, has been estimated to exceed $88 \%$ (1). For this reason, there have been a number of MI gene mapping studies; in human these include Zielenski et al. (2), Blackman et al. (1), and Dorfman et al. (3). However there has been a general lack of consistency in findings across the published studies. Possible explanations include false positive results, or substantial genetic heterogeneity. As part of the International Cystic Fibrosis Gene Modifier Consortium we set out to better understand the genetic basis of MI.

The International Gene Modifier Consortium consists of data ascertained as part of the University of North Carolina/Case Western Reserve extremes of lung phenotype study, the Johns Hopkins University CF Twin and Sibling study, the Canadian consortium for gene modifiers population-based study, and the French CF population-based study. Using the North American data collections we conducted a genome-wide association study (GWAS) of $\mathrm{MI}$ in $\mathrm{CF}$, comprised of 3,763 CF patients with severe CFTR genotypes, genotyped at 543,927 single nucleotide polymorphisms (SNPs) across the genome, to identify variants associated with MI. Five genome-wide significant SNPs $\left(\mathrm{p}<5 \times 10^{-8}\right)$ were identified in two genomic regions that include the genes SLC26A9 (min p $=9.88 \times 10^{-9}$ ) on chromosome 1 and SLC6A14 (min $\mathrm{p}=1.28 \times 10^{-12}$ ) on chromosome $\mathrm{X}$; neither of which had been previously identified in other MI modifier studies. The association with these genes was replicated in an independent collection of North American data from the consortium, and in the French CF cohort $(p=0.001$ and $\mathrm{p}=0.0001$ for SLC6A14 and SLC26A9, respectively). None of the associated SNPs are in the coding regions of these genes, but rather are located just upstream of their respective transcription start sites, suggesting that the variants may affect gene expression.

The associated SNPs, however, account for less than 5\% of the phenotypic variance in MI, suggesting other genetic factors are yet to be identified, and that there is potential for substantial genetic heterogeneity. To try to identify additional genes contributing to the MI variance, we developed the Hypothesis-Driven GWAS, which provides a way to incorporate hypothesized mechanisms for MI based on known biology into the assessment of genetic association. The Hypothesis-driven GWAS is based on the observation that CF disease is caused by impaired fluid and electrolyte flux at the epithelial interfaces of all CF- affected organs including the airway, intestine, pancreas, liver and vas deferens. In all these organs, the single cell epithelial layer is unique in that it forms a highly selective and tight barrier between body organ and ductal interfaces. Epithelial "function" is achieved by cell polarization whereby many determinants and regulators of fluid, solute and ion transport, including the CFTR ion channel reside specifically at the apical membrane, with contributing features from basal and lateral surfaces. We hypothesized that with loss of CFTR, other transporters that result in variation in any residual or adapted epithelial functions contributed by apical membrane constituents could modify CF phenotypes, such as MI.

We tested the apical hypothesis, by assigning SNPs of genes that encode proteins that localize to the apical plasma membrane to a high priority group and comparing their association evidence to those genes that are not 
localized to the apical plasma membrane. Hypothesisdriven GWAS was implemented using two statistical procedures. First, using the prioritized apical membrane list, the stratified false discovery rate control (SFDR) (4) was applied to the data to re-evaluate the initial association evidence for any given SNP at the genome-wide level. Then a permutation-based test was used to determine the statistical significance of the apical hypothesis as a whole, testing all high priority SNPs jointly to assess whether a preponderance of apical constituents contribute to MI susceptibility.

A list of 157 genes was annotated as localized to the apical plasma membrane using GO Consortium data (5). This list included SLC26A9 but not SLC6A14 despite it being localized to the brush border membrane. After applying the GWAS-HD, we observed that the group of constituents from the apical plasma membrane was associated with $\mathrm{MI}$ $(\mathrm{p}=0.0002)$, and this association was replicated in the independent French cohort $(\mathrm{p}=0.022)$. The SFDR indicated that SNPs in two additional genes, SLC9A3 and ATP2B2, also reached genome-wide significance after re-prioritization with the apical hypothesis. The gene-based analysis in the French cohort confirmed the SLC9A3 association. Using multivariate regression modeling we were able to estimate that 36 different genes from the apical plasma membrane gene list, including SLC9A3 and SLC26A9, are associat- ed with MI. These genes along with SLC6A14 can account for $\sim 17 \%$ of the phenotypic variance.

To conclude, the multiple associated genes suggest the genetic basis of MI is quite heterogeneous, which might explain the lack of consensus across MI modifier studies to date. Thus far, we have been able to account for $\sim 17 \%$ of the phenotypic variability, However, the predicted heritability of MI exceeds $88 \%$, suggesting that there are many more modifiers to be found.

References: 1. Blackman SM, Deering-Brose R, McWilliams R, et al. Relative contribution of genetic and nongenetic modifiers to intestinal obstruction in CF. Gastroenterology. 2006;131:1030-9.

2. Zielenski J, Corey M, Rozmahel R, et al. Detection of a cystic fibrosis modifier locus for meconium ileus on human chromosome 19q13. Nat Genet. 1999;22:128-9.

3. Dorfman R, Li W, Sun L, et al. Modifier gene study of meconium ileus in $\mathrm{CF}$ : statistical considerations and gene mapping results. Hum Genet. 2009;126:763-78.

4. Yoo YJ, Bull SB, Paterson AD, et al. Were genomewide linkage studies a waste of time? Exploiting candidate regions within genome-wide association studies. Genet Epidemiol. 2010;34:107-18.

5. Ashburner M, Ball CA, Blake JA, et al. Gene ontology: tool for the unification of biology. The Gene Ontology Consortium. Nat Genet. 2000;25:25-9.

\title{
S19.2 \\ PATHOPHYSIOLOGIC STUDIES OF INTESTINAL DYSFUNCTION
}

\author{
Robert De Lisle, Ph.D. \\ Anatomy \& Cell Biology, Univ. of Kansas School Medicine, Kansas City, KS, USA
}

The intestines are affected early in CF, often with clinical manifestations at birth. Additionally, intestinal dysfunction continues throughout life in CF. CF intestinal issues range from the very specific life threatening problem of intestinal obstruction, to those that primarily affect quality of life, such as bloating and flatulence. The impact of $\mathrm{CF}$ on the intestines contributes to poor nutrition and failure to thrive, which are strongly associated with worse outcomes. While loss of exocrine pancreatic function is the major contributor to impaired digestion in $\mathrm{CF}$, even with optimal pancreatic enzyme replacement therapy, nutritional problems are still common which points to impaired intestinal function.

Loss of CFTR function results in decreased volume of fluid with abnormal electrolyte composition on the epithelial surfaces and in the lumens of affected organs. As in the airways, pathogenesis in the CF intestine appears to follow the same sequelae as a consequence of loss of CFTR activity: mucus accumulation/obstruction, microbial infection, and inflammation.

There have been relatively few investigations of the human CF small intestine, mostly due to its inaccessibility. Much of our current understanding of CF pathogenesis in the intestine is a result of studies using genetically modified mouse models of CF. Use of transgenic mice has contributed greatly to our understanding of normal electrolyte transport mechanisms in the intestine including the roles of CFTR (1). The major phenotype of CF mice is the lethal obstruction of the distal small intestine. There are over a dozen strains of CF mice, including total knockout of the Cftr gene as well as those with specific mutations in CFTR known to cause disease in humans (2). Comparison of these different CF strains reveals $10-15 \%$ of normal CFTR function is sufficient to avoid intestinal obstruction which has important implications for therapies in development such as CFTR small molecule correctors. The most severely affected mouse strain is a conventional knockout which totally lacks $C f t r$ mRNA and protein expression (3). Half of such CF mice die before weaning and most of the survivors die shortly after weaning as they begin to consume solid food. Post-weaning intestinal obstruction can be prevented by feeding the mice a liquid diet (4) or giving them an osmotic laxative solution to drink (5).

The initiating event in CF pathogenesis in the intestine is due to the abnormal fluid produced when CFTR is dysfunctional; secreted epithelial fluid in CF is lower in 
volume and lacking normal bicarbonate ion levels. The importance of these secretions in CF is shown by the fact that improving the hydration state of the gut using liquid food (4), orally administered osmotic laxative (5), or crossing CF mice with transgenic mice that have disruptions in electrolyte absorption pathways (which contribute to reduced fluid volumes in $\mathrm{CF}$ ), can prevent intestinal obstruction $(6,7)$.

Changes in the $\mathrm{CF}$ mouse small intestine largely confirm those reported in human $\mathrm{CF}$, namely: accumulation of mucus, bacterial overgrowth, a mild immune response, altered eicosanoid metabolism, slow intestinal transit, fat malabsorption, and impaired mucosal barrier function. $\mathrm{CF}$ mice, also similar to human $\mathrm{CF}$, are of low body weight indicating maldigestion and malnutrition.

When examined histologically, the CF intestine exhibits excessive mucus which physically blocks the intestinal crypt lumen and accumulates along the intestinal surfaces. Blockage of the crypt lumen has been proposed to prevent access of Paneth cell derived antimicrobials to the intestinal lumen, and CF mice are more susceptible to infection with pathogenic bacteria (8). The mucus that accumulates in the $\mathrm{CF}$ mouse intestinal lumen becomes heavily colonized with colonic type bacteria (9), and bacterial overgrowth happens within 4 days of birth (10). There is also a loss of bacterial diversity in the CF mouse small intestine $(9,10)$. Both the change in composition of bacterial species and their overgrowth are forms of microbial dysbiosis. Microbial dysbiosis has wide-ranging effects not only on intestinal function, but also on the development and behavior of the systemic immune system (11). The implications of gut microbial dysbiosis in CF are not well understood.

Accompanying microbial dysbiosis of the $\mathrm{CF}$ mouse small intestine, there is an activation of innate immune defenses, which includes large infiltrations of neutrophils and mast cells (12). Microarray analysis showed that there are many changes in gene expression in the CF mouse small intestine, a lot of which are components of the innate immune system (12). Among the altered genes are several involved in eicosanoid metabolism. There are increases in some phospholipase A2s, which cleave membrane phospholipids to produce arachidonic acid, the precursor for a variety of eicosanoids. Also, there is decreased expression of the major prostaglandin degrading enzymes, prostaglandin dehydrogenase $(H p g d)$ and prostaglandin reductase (Ptgrl). Coincident with these changes in gene expression there are elevated levels of $\mathrm{PGE}_{2}$ and $\mathrm{PGF}_{2} \mathrm{a}$ (13). An important functional significance of elevated $\mathrm{PGE}_{2}$ is that this eicosanoid has a dominant relaxant effect on intestinal circular smooth muscle, thus providing a mechanistic explanation for decreased small intestinal motility that occurs in CF (14).
Most strains of CF mice have impaired weight gain even though these mice are all pancreatic sufficient. This indicates there are intestinal issues contributing to CF malnutrition. A major nutritional issue in human $\mathrm{CF}$ is poor fat digestion/assimilation. Similar to human $\mathrm{CF}, \mathrm{CF}$ mice have impaired lipolysis as well as impaired post-lipolytic uptake of fatty acids, and the former was improved by inhibition of gastric acid (15). The mechanisms responsible for impaired fat assimilation are only partly known and a complete understanding of fat maldigestion in $\mathrm{CF}$ remains an important goal.

CF mice have been used to test therapeutic approaches to improve intestinal function. Direct eradication of bacterial overgrowth using oral broad spectrum antibiotics reduces inflammation, decreases mucus accumulation, improves barrier function, and improves body weight gain $(9,16)$. Interestingly, antibiotics do not improve intestinal motility in CF mice and, in fact, impair motility in wild type mice which is accompanied by changes in eicosanoid metabolism similar to that of control CF mice (14). These observations illustrate the complex interplay between the microbiota and gut function, and serve as a cautionary tale about potential unintended consequences of manipulating the gut microbiota.

Treatment of CF mice with laxative at weaning sufficiently improves luminal hydration such that there is a significant decrease in mucus accumulation, eradication of bacterial overgrowth, less inflammation, improved motility, improved barrier function, and a small increase in weight gain $(10,17)$. Laxatives are commonly used in the CF clinic to treat GI symptoms, but the mouse data suggest that chronic laxative use may be a good prophylaxis for CF patients. This idea needs further investigation.

We will continue to learn important lessons about intestinal dysfunction in CF using mouse models, as well as from newly developed CF pigs and ferrets. One goal is to discover how to improve intestinal function in $\mathrm{CF}$ which is expected to further contribute to greater longevity and quality of life. Also, as the pathogenesis of CF intestinal disease has many similarities with $\mathrm{CF}$ airway disease, novel therapeutic approaches that benefit the intestine may lead to analogous airway therapies. Because of the central role the normal gut microbiota has for systemic immune system development and function, there is also a real potential that therapies that improve intestinal function in CF may have broader benefits such as reducing the hyperresponsive airway immune responses characteristic of this disease.

References: 1. Seidler U, Singh A, Chen M, et al. Exp Physiol. 2009;94:175-9.

2. Guilbault C, Saeed Z, Downey GP, et al. Am J Respir Cell Mol Biol. 2007;36:1-7. 
3. Snouwaert JN, Brigman KK, Latour AM, et al. Science 1992;257:1083-8.

4. Eckman EA, Cotton CU, Kube DM, et al. Am J Physiol Lung Cell Mol Physiol. 1995;269:L625-30.

5. Clarke LL, Gawenis LR, Franklin CL, et al. Lab Anim Sci. 1996;46:612-8.

6. Walker NM, Simpson JE, Levitt RC, et al. J Pharmacol Exp Ther. 2005;317:275-83.

7. Bradford EM, Sartor MA, Gawenis LR, et al. Am J Physiol Gastrointest Liver Physiol. 2009;296:G886-98.

8. Clarke LL, Gawenis LR, Bradford EM, et al. Am J Physiol Gastrointest Liver Physiol. 2004;286:G1050-8.

9. Norkina O, Burnett TG, De Lisle RC. Infect Immun. 2004;72:6040-9.
10. Canale-Zambrano JC, Auger ML, Haston CK. Am J Physiol Gastrointest Liver Physiol. 2010;299:G381-90.

11. Sekirov I, Russell SL, Antunes LC, et al. Physiol Rev. 2010;90:859-904.

12. Norkina O, Kaur S, Ziemer D, et al. Am J Physiol Gastrointest Liver Physiol. 2004;286:G1032-41.

13. De Lisle RC, Meldi L, Flynn M, et al. J Pediatr Gastroenterol Nutr. 2008;47:406-16.

14. De Lisle RC, Sewell R, Meldi L. Neurogastroenterol Motil. 2010;22:341-e87.

15. Bijvelds MJ, Bronsveld I, Havinga R, et al. Am J Physiol Gastrointest Liver Physiol. 2005;288:G646-53.

16. De Lisle RC, Roach EA, Norkina O. J Pediatr Gastroenterol Nutr. 2006;42:46-52.

17. De Lisle RC, Roach E, Jansson K. Am J Physiol Gastrointest Liver Physiol. 2007;293:G577-84.

\title{
S19.3 ROLE OF BICARBONATE IN INTESTINAL MUCUS SECRETION
}

\author{
Ning Yang ${ }^{1}$ and Paul M. Quinton, Ph.D., ${ }^{1,2}$ \\ 1. Pediatrics, Univ. of California San Diego, La Jolla, CA, USA; 2. Biomedical Sciences, Univ. of California, \\ Riverside, Riverside, CA, USA
}

The mucus layer coating the gastrointestinal tract is one of the first lines of innate host defense. However, in cystic fibrosis (CF), a hallmark of the disease is the accumulation of abnormally thick and sticky mucus in the lung, intestine, and various other exocrine organs. Although the accumulation of thick mucus is thought likely to play a central role in the development of $\mathrm{CF}$, how mutations in the CFTR gene lead to mucus accumulation has not been determined.

Why $\mathrm{HCO}_{3}{ }^{-}$? Several hypotheses are proposed to explain the pathogenesis of abnormally thick mucus in CF. Probably the most prevalent is excessive $\mathrm{Na}^{+}$and fluid absorption that dehydrates mucus due to unregulated epithelial $\mathrm{Na}^{+}$channel $(\mathrm{ENaC})$ activity (1). However, it is very difficult to extend this rationale from the airways to other $\mathrm{CF}$ affected organs, where $\mathrm{ENaC}$ is not expressed, for example, the small intestine. Impaired $\mathrm{HCO}_{3}{ }^{-}$secretion in major $\mathrm{CF}$-affected organs such as pancreas and small intestine has been documented for many years (2-4), but the possible involvement of $\mathrm{HCO}_{3}^{-}$with mucus formation has received little attention. The major constituents of normal mucus are mucin glycoproteins, which are large, heavily glycosylated proteins with a defining feature of tandem repeating sequences of amino acids. Intracellularly, the $\mathrm{Ca}^{2+}$ and $\mathrm{H}^{+}$cations shield the high density of fixed anionic sites on the oligosaccharides that surround the protein cores of the mucin monomers $(5,6)$. We hypothesized that $\mathrm{HCO}_{3}^{-}$as an important biological buffer may play a crucial role in unpackaging and expanding mucins from condensed granules to the final mucus substance by neutralizing $\mathrm{H}^{+}$and /or sequestering $\mathrm{Ca}^{2+}$.

Can $\mathrm{HCO}_{3}^{-}$help mucus release in the small intestine? To validate our hypothesis, we designed experiments to determine the impact of $\mathrm{HCO}_{3}^{-}$on mucus release. We made a simple temperature-controlled mucus perfusion system. Mouse intestinal segments were perfused luminally with glucose-free Ringers solution. The perfusate was collected and assayed by WGA-lectin binding or by PAS staining of the filtrand retained on Immobilon-P film (7). After testing several agonists, we found that serotonin (5HT) and prostaglandin E2 (PEG2) are two potent mucus secreting stimulants. Then, stimulated mucus release was tested in the presence and absence of $\mathrm{HCO}_{3}{ }^{-}$or different inhibitors. After removing $\mathrm{HCO}_{3}{ }^{-}$from the bath solution, released mucus in perfusate was significantly decreased compared to the $\mathrm{HCO}_{3}{ }^{-}$Ringers solution. Further, the $\mathrm{Na}^{+}$$\mathrm{HCO}_{3}{ }^{-}$contransporter (NBC) inhibitor, DIDS, also markedly reduce 5-HT and PGE2-induced mucus release. These results suggest that $\mathrm{HCO}_{3}{ }^{-}$is crucial for normal mucus release. We observed that PGE2 and 5-HT did not induce significant mucus release either in CFTR knock-out mice or in the presence of CFTR inhibitor, glyH-101, indicating that normal mouse intestinal mucus release requires CFTR-dependent $\mathrm{HCO}_{3}{ }^{-}$secretion (7).

Is the $\mathrm{HCO}_{3}^{-}$-dependent mucus release due to $\mathrm{HCO}_{3}^{-}$-dependent fluid secretion? In addition to $\mathrm{HCO}_{3}^{-}$, fluid secretion also appears to be crucial for mucus transport (7-9), which raises a possibility that 
increased mucus release in the presence of $\mathrm{HCO}_{3}{ }^{-}$may be due simply to a $\mathrm{HCO}_{3}^{-}$dependent fluid lavage effect. To determine whether decreased mucus release in the absence of $\mathrm{HCO}_{3}{ }^{-}$is due to decreased $\mathrm{HCO}_{3}{ }^{-}$-dependent fluid secretion, we compared fluid secretion rates from closed intestinal sacs with and without $\mathrm{HCO}_{3}{ }^{-}$in the bath solutions. Increases and decreases in weight were interpreted as fluid secretion and absorption, respectively. 5-HT or PGE2 markedly increased fluid secretion, which was completely blocked by bumetanide $\left(\mathrm{a} \mathrm{Na}^{+}-\right.$ $\mathrm{K}^{+}-2 \mathrm{Cl}^{-}$contransporter inhibitor). Removing $\mathrm{HCO}_{3}^{-}$ from the bath had no detectable effect on basal or stimulated fluid secretion. Likewise, DIDS did not reduce fluid secretion. These findings indicate that the $\mathrm{HCO}_{3}^{-}$dependent increase in stimulated mucus release is unlikely due to $\mathrm{HCO}_{3}{ }^{-}$dependent fluid secretion and lavage effect (7).

How does $\mathrm{HCO}_{3}^{-}$help mucus release? To test our hypothesis that $\mathrm{HCO}_{3}{ }^{-}$enhances mucus release by neutralizing $\mathrm{H}^{+}$(changing $\mathrm{pH}$ ) and/or sequestering $\mathrm{Ca}^{2+}$, porcine stomach mucin was dissolved with $\mathrm{Ca}(\mathrm{OH})_{2}$ and divided into equal aliquots. Equal moles of $\mathrm{NaCl}$ and $\mathrm{NaHCO}_{3}$ were added to each aliquot, respectively. $\mathrm{HCO}_{3}{ }^{-}$decreased both the $\mathrm{pH}$ and $\left[\mathrm{Ca}^{2+}\right]$ dramatically. Subsequently, each mucus solution was filtered through an Immobilon-P membrane. The weight of mucus retained on the filter membrane from the mucus solution treated with $\mathrm{NaCl}$ was significantly higher than that treated with $\mathrm{NaHCO}_{3}$, indicating that $\mathrm{HCO}_{3}{ }^{-}$reduced the size of mucus aggregates in solution. Furthermore, to better define the role of $\mathrm{pH}$ and $\left[\mathrm{Ca}^{2+}\right]$ in $\mathrm{HCO}_{3}^{-}$-induced mucus disaggregation, we replaced the $\mathrm{Ca}^{2+}$ with $\mathrm{NaOH}$ equimolar for $\mathrm{OH}^{-}$in the initial mucus solution, and then treated aliquots of the resulting $\mathrm{NaOH}$ mucus solution with equimolar $\mathrm{NaCl}$ or $\mathrm{NaHCO}_{3}$. The $\mathrm{pH}$ of the mucus solution treated with $\mathrm{NaHCO}_{3}$ decreased significantly, but the amount of mucus retained on the filter membrane was the same for mucus solutions with $\mathrm{NaCl}$ or with $\mathrm{NaHCO}_{3}$. In this experiment, the initial $\mathrm{pH}$ of the aliquots of $\mathrm{NaOH}$ mucus solutions were similar to the mucus solutions with $\mathrm{Ca}(\mathrm{OH})_{2}$, but differed in the concentration of $\left[\mathrm{Ca}^{2+}\right]$. These results indicate that the disaggregating effect of $\mathrm{HCO}_{3}{ }^{-}$is probably due to sequestering the $\left[\mathrm{Ca}^{2+}\right]$ from the mucus (10).

To further prove that $\mathrm{HCO}_{3}{ }^{-}$disaggregates mucus by decreasing $\left[\mathrm{Ca}^{2+}\right]$ in mucus, we collaborated with Prof. Wei-Chun Chin and Dr. Eric Y. T. Chen at the University of California, Merced. Optical emission spectrometry was used to quantify the relative amount of bound $\mathrm{Ca}^{2+}$ displaced from mucus after adding $\mathrm{HCO}_{3}^{-}$. Porcine stomach mucus with $\mathrm{Ca}^{2+}$ was added with $\mathrm{HCO}_{3}{ }^{-}$and assayed at increasing intervals of time. The mucus sample was filtered through an Isopore membrane and redissolved in $1 \% \mathrm{HNO} 3$ solution. $\mathrm{As}^{\mathrm{HCO}_{3}}{ }^{-}$incubation time increased, the amount of mucus-bound $\mathrm{Ca}^{2+}$ decreased, showing that $\mathrm{HCO}_{3}{ }^{-}$readily sequesters bound $\mathrm{Ca}^{2+}$ from mucus.
To show that $\mathrm{HCO}_{3}{ }^{-}$can directly disperse aggregated mucus gels, $\mathrm{Ca}^{2+}$ was added to porcine gastric mucus and equilibrated to form mucus gels. $\mathrm{HCO}_{3}^{-}$dispersed aggregated mucus gel particles, and significantly decreased the mucus gel in size. Addition of EGTA $\left(\mathrm{Ca}^{2+}\right.$ chelator $)$ had a similar effect. Again, these data indicate that $\mathrm{HCO}_{3}{ }^{-}$ likely disperses $\mathrm{Ca}^{2+}$ cross-linked mucus gels by sequestering free as well as $\mathrm{Ca}^{2+}$ bound in mucus networks (10).

Conclusions. Our results suggest that: 1) Normal intestinal mucus release requires CFTR-dependent $\mathrm{HCO}_{3}{ }^{-}$ secretion; and 2) $\mathrm{HCO}_{3}^{-}$-dependent mucus release is probably not due to a lavage effect of $\mathrm{HCO}_{3}{ }^{-}$-dependent fluid secretion, but due to mucus gel dispersion upon removing $\mathrm{Ca}^{2+}$ from the mucus matrix. These findings provide insights for the pathogenesis and perhaps new directions for treatments in CF. Aside from $\mathrm{Na}^{+}$absorption or $\mathrm{Cl}^{-}$ secretion, correcting the defective management of $\mathrm{HCO}_{3}{ }^{-}$ in CF may be necessary for optimal therapies in CF.

Acknowledgments. The authors' work is supported by Elizabeth Nash Memorial Fellowship, CFRI; MCCC-Cystic Fibrosis Foundation; NIH-RO1 HL084042; Nancy Olmsted Endowment. The authors thank Dr. Hui Dong for the use of his $\mathrm{pH}$ stat equipment and his helpful discussions.

References: 1 . Boucher RC. Cystic fibrosis: a disease of vulnerability to airway surface dehydration. Trends Mol Med. 2007;13:231-40.

2. Gaskin KJ, Durie PR, Corey M, et al. Evidence for a primary defect of pancreatic $\mathrm{HCO} 3$-secretion in cystic fibrosis. Pediatr Res. 1982;16:554-7.

3. Seidler U, Blumenstein I, Kretz A, et al. A functional CFTR protein is required for mouse intestinal cAMP-, cGMP- and $\mathrm{Ca}(2+)$-dependent HCO3- secretion. J Physiol. 1997;505:411-23.

4. Clarke LL, Harline MC. Dual role of CFTR in cAMP-stimulated HCO3- secretion across murine duodenum. Am J Physiol. 1998;274:G718-26.

5. Verdugo P, Aitken M, Langley L, et al. Molecular mechanism of product storage and release in mucin secretion. II. The role of extracellular $\mathrm{Ca}++$. Biorheology. 1987;24:625-33.

6. Verdugo P, Deyrup-Olsen I, Aitken M, et al. Molecular mechanism of mucin secretion: I. The role of intragranular charge shielding. J Dent Res. 1987;66:506-8.

7. Garcia MA, Yang N, Quinton PM. Normal mouse intestinal mucus release requires cystic fibrosis transmembrane regulator-dependent bicarbonate secretion. J Clin Invest. 2009;119:2613-22.

8. Yamaya M, Finkbeiner WE, Widdicombe JH. Altered ion transport by tracheal glands in cystic fibrosis. Am J Physiol. 1991;261:L491-4.

9. Joo NS, Cho HJ, Khansaheb M, et al. Hyposecretion of fluid from tracheal submucosal glands of CFTRdeficient pigs. J Clin Invest. 2010;120:3161-6.

10. Chen EY, Yang N, Quinton PM, et al. A new role for bicarbonate in mucus formation. Am J Physiol Lung Cell Mol Physiol. 2010;299:L542-9. 


\title{
S19.4 \\ CLINICAL MANIFESTATIONS OF CF IN THE INTESTINE
}

\author{
Daniel Gelfond, M.D. \\ Pediatric Gastroenterology \& Nutrition, State Univ. of NY at Buffalo School of Medicine \& Biomedical \\ Sciences/Children's Hospital of Buffalo, Buffalo, NY, USA
}

Introduction. CFTR is found throughout the entire gastrointestinal (GI) tract. Its dysfunction contributes to the intestinal problems in patients with $\mathrm{CF}$ as well as pancreatic and hepatobiliary disease. Clinical symptoms related to intestinal manifestations are not exclusive to the CF population, however patients with $\mathrm{CF}$ often have higher prevalence of intestinal complaints.

In CF patients the incidence of GERD is estimated to be 6-8 times higher when compared to the general population. Children with $\mathrm{CF}$ tend to have a higher prevalence of GERD when compared to the adult CF population (1). The principal mechanism causing GERD is transient (inappropriate) relaxation of the lower esophageal sphincter. Esophageal pH recordings in children with $\mathrm{CF}$ have a higher esophageal exposure to gastric acid along with increased symptoms of regurgitation and heartburn when compared to non-CF children (2). Long-term complications of GERD such as esophagitis and esophageal stricture is more common in CF children and adults (3). There is a well recognized relationship between GERD and chronic obstructive pulmonary disease (4). Despite supporting evidence for aggressive GERD therapy to improve pulmonary status, there is poor correlation between severity of GERD and FEV1 or FVC in adult patients (5). Use of proton pump inhibitors has become the first line of therapy in reducing acid production.

Meconium ileus (MI) is an acute condition of newborn infants due to dehydrated thickened meconium that causes mechanical obstruction in the distal ileum along with failure to pass meconium within the first 24-48 hours after birth. Approximately $20 \%$ of infants with CF present with meconium ileus at birth. MI is very suggestive of $\mathrm{CF}$, yet up to half of infants presenting with MI do not carry a diagnosis of CF (6). Non-CF diseases associated with abnormal gut motility, such as Hirschsprung disease and chronic intestinal pseudo-obstruction, have been associated with MI-like disease, suggesting that decreased peristalsis may allow for increased resorption of water, thus favoring the development of MI. Mouse models of CF have shown that severe neonatal bowel obstruction can occur despite normal pancreatic function. These models also highlight that defective $\mathrm{HCO}_{3}^{-}$excretion leads to an acidic and dehydrated luminal environment. Lack of $\mathrm{HCO}_{3}^{-}$likely leads to compacted, dehydrated mucus that contributes to MI. Abnormal intestinal motility may also contribute to the development of MI. Furthermore, there is growing evidence for multiple loci modifier gene involvement in the pathogenesis of MI. Medical management with enema irrigation is successful in up to fifty percent of infants. Some infants with MI

require urgent surgical interventions either secondary to failed medical management or complications of enema therapy (7). Overall outcomes of medical and surgical intervention of infants with MI are encouraging. Understanding of the relationship between MI and genetic predisposition as well as environmental impact on infants with CF clearly deserves further investigations.

Distal intestinal obstruction syndrome (DIOS) occurs in patients of all ages including adults with $\mathrm{CF}$ and is predominantly localized to the ileocaecum. Accumulation of viscid fecal material with strong adhesion to villi and crypts of the mucosa is a hallmark of this syndrome. Clinically it is differentiated from constipation by an acute onset of symptoms suggestive of partial or complete intestinal obstruction. DIOS can also present as intermittent symptoms of abdominal pain and distention likely attributed to a partial breakdown and re-accumulation of mucofeculent material over a strongly adherent mucoid mass. Radiographic images suggestive of right lower quadrant fecal loading along with clinical symptoms and physical findings of a palpable mass is sufficient to establish a diagnosis pending exclusion of other surgical and medical causes of right lower quadrant abdominal pain with clinical signs of obstruction (8). DIOS is seen more frequently with pancreatic insufficient patients as well as those with a prior history of MI or prior episodes of DIOS (8). In European studies, prevalence of DIOS was found to be up to seven times greater in adult patients compared to pediatric patients with $\mathrm{CF}$ (9). Other mechanisms such as intestinal dysmotility, mucosal inflammation, tissue hypertrophy, fat malabsorption and defective chloride and water secretion into gut lumen are likely to contribute to obstructive process and collectively contribute to DIOS (8). Pharmacologic management is centered on luminal rehydration with osmotic stool softeners to mobilize the mucus mass and relieve obstruction. Enemas and nasogastric decompression can be used in cases of severe obstruction. Surgical intervention remains the last resort once medical management fails or there are signs of bowel ischemia (8). Emphasis is also placed on prevention of recurrent episodes of DIOS by adherence with pancreatic enzyme replacement therapy, hydration and routine stool softeners.

Constipation is a frequent complaint in patients with $\mathrm{CF}$ and likely shares some of the underlying mechanism with DIOS such as dysmotility and decreased water secretion secondary to CFTR defect. Similar to DIOS, fat malabsorption and prior history of MI were noted to be independent risk factors in development of constipation in the pediatric CF patients (10). Although overall 
incidence of constipation in patients with $\mathrm{CF}$ did not differ from the general population (32\% vs. $34-37 \%$ respectively), it was noted to be 1.5 times as prevalent in patients with pancreatic insufficiency when compared to the pancreatic sufficient patients (11). Despite these observations, there is no correlation between the dose of pancreatic enzymes and self-reported constipation in patients with pancreatic insufficiency (11). In contrast to the general population where the likely cause of constipation in a pediatric age group is deficiency in dietary fiber and inadequate fluid intake, these factors were not associated with constipation in children with CF (10). Therapeutic options in the $\mathrm{CF}$ patient with constipation are not different from the medical management of constipation in general population. Osmotic stool softeners are usually first line of treatment along with infrequent supplementation with enemas or stimulant laxatives.

In CF patients, thick mucus secretions along with intestinal dysmotility can predispose patients to intestinal stasis facilitating development of small bowel bacterial overgrowth (SBBO). Bacterial overgrowth within the segments of the small intestine produce toxic byproducts and metabolites often leading to enterocyte damage, malabsorption and malnutrition. Our current understanding of the pathophysiology of SBBO in CF has been facilitated by studies using the CF mouse model (12). SBBO can present with symptoms of abdominal pain, distention and diarrhea. There are significant limitations in studying $\mathrm{SBBO}$ in patients. Diagnostic modalities such as imaging, culture of duodenal aspirates and breath hydrogen testing all have limitations. Empiric therapy with enteric antibiotics and probiotics is frequently initiated to suppress and potentially eradicate bacterial overgrowth in the small bowel.

References: 1. Malfroot A, Dab I. New insights on gastro-oesophageal reflux in $\mathrm{CF}$ by longitudinal follow up. Arch Dis Child. 1991;66:1339-45.
2. Scott RB, O’Loughlin EV, Gall DG. Gastroesophageal reflux in patients with CF. J Pediatr. 1985;106:223-7.

3. Bendig DW, Seilheimer DK, Wagner ML, et al. Complications of gastroesophageal reflux in patients with cystic fibrosis. J Pediatr. 1982;100:536-40.

4. Rabinovich RA, MacNee W. Chronic obstructive pulmonary disease and its comorbidities. Br J Hosp Med (Lond). 2011;72:137-45.

5. Sabati AA, Kempainen RR, Milla CE, et al. Characteristics of gastroesophageal reflux in adults with CF. J Cyst Fibros. 2010;9:365-70.

6. Gorter R, Karimi A, Sleeboom C, et al. Clinical and genetic characteristics of meconium ileus in newborns with and without CF. J Pediatr Gastroenterol Nutr. 2010;50:569-72.

7. Karimi A, Gorter RR, Sleeboom C, et al. Issues in the management of simple and complex meconium ileus. Pediatr Surg Int. 2011;Apr 22 [Epub ahead of print].

8. Colombo C, Ellemunter H, Houwen R, et al., Guidelines for the diagnosis and management of distal intestinal obstruction syndrome in cystic fibrosis patients. J Cyst Fibros. 2011;10(S2):S24-8.

9. Andersen HO, Hjelt K, Waever E, et al. The agerelated incidence of meconium ileus equivalent in a $\mathrm{CF}$ population: the impact of high-energy intake. J Pediatr Gastroenterol Nutr. 1990;11:356-60.

10. van der Doef HPJ, Kokke FT, Beek FJ, et al. Constipation in pediatric CF patients: An underestimated medical condition. J Cyst Fibros. 2010;9:5963.

11. Baker SS, Borowitz D, Duffy L, et al. Pancreatic enzyme therapy and clinical outcomes in patients with CF. J Pediatr. 2005;146:189-93.

12. De Lisle RC. Altered transit and bacterial overgrowth in the CF mouse small intestine. Am J Physiol Gastrointest Liver Physiol. 2007;293:G104-11.

\title{
S20.1 \\ WHAT DO NEW ANIMAL MODELS TEACH US ABOUT LUNG INFECTIONS IN CF?
}

\author{
David A. Stoltz, M.D., Ph.D. \\ Internal Medicine, Univ. of Iowa Hospitals \& Clinics, Iowa City, IA, USA
}

Despite significant advances in our understanding of $\mathrm{CF}$ disease pathogenesis and therapeutic interventions, $\mathrm{CF}$ remains a potentially lethal disease. Lung disease causes most of the morbidity and mortality in cystic fibrosis (CF) (1). Understanding the pathogenesis of the lung disease has been hindered, however, by the lack of an animal model with characteristic features of CF. We chose pigs for the development of a new CF model, because they are more similar to humans than are mice in terms of anatomy, physiology, immune system, biochemistry, life span, size, and genetics $(2,3)$. We now know that, within months of birth, CF pigs (both CFTR/- and CFTR $\Delta F 508 / \Delta F 508$ ) spontaneously develop features typical of human $C F$ lung disease, including airway inflammation, infection, remodeling, mucus accumulation, and airway obstruction (4-6). Findings from the CF pig lung have provided insight into $\mathrm{CF}$ disease pathogenesis. First, CF pig lungs contain multiple bacterial 
species, suggesting that the lungs of CF pigs have a host defense defect against a wide spectrum of bacteria. Second, the number of bacteria in the $\mathrm{CF}$ pig lung is variable over time, similar to humans with $\mathrm{CF}$, suggesting that not all host defenses are impaired. Third, the CF pig has provided additional insight regarding the temporal and causal relations between inflammation and infection in the CF lung. Newborn CF pig lungs show no inflammation but are less often sterile than controls. Fourth, after introduction of bacteria into $\mathrm{CF}$ pig lungs, pigs with $\mathrm{CF}$ fail to eradicate bacteria as effectively as wild-type pigs. Finally, ion transport studies in CFTR-/- newborn nasal and tracheal/bronchial epithelia show markedly reduced $\mathrm{Cl}^{-}$and $\mathrm{HCO}_{3}{ }^{-}$transport (7). Yet, lack of CFTR does not increase transepithelial $\mathrm{Na}^{+}$or liquid absorption or reduce periciliary liquid depth. These results suggest that impaired bacterial eradication is the pathogenic event that initiates a cascade of inflammation and pathology in CF lungs. Furthermore, these studies suggest that reduced anion permeability, and not increased $\mathrm{Na}^{+}$transport, initiates CF airway disease.

References: 1. Rowe SM, Miller S, Sorscher EJ. Cystic fibrosis. N Engl J Med. 2005;352:1992-2001.
2. Ibrahim Z, Busch J, Awwad M, et al. Selected physiologic compatibilities and incompatibilities between human and porcine organ systems. Xenotransplantation. 2006;13:488-99.

3. Rogers CS, Abraham WM, Brogden KA, et al. The porcine lung as a potential model for CF. Am J Physiol Lung Cell Mol Physiol. 2008;295:L240-L263.

4. Rogers CS, Stoltz DA, Meyerholz DK, et al. Disruption of the CFTR gene produces a model of cystic fibrosis in newborn piglets. Science. 2008;321:1837-41.

5. Stoltz DA, Meyerholz DK, Pezzulo AA, et al. Cystic fibrosis pigs develop lung disease and exhibit defective bacterial eradication at birth. Sci Trans Med. 2010;2:29ra31.

6. Ostedgaard LO, Meyerholz DK, Chen JH, et al. The $\Delta$ F508 mutation causes CFTR misprocessing and CF-like disease in pigs. Science Transl Med. 2011;3:74ra24.

7. Chen JH, Stoltz DA, Karp PH, et al. Loss of anion transport without increased sodium absorption characterizes newborn porcine cystic fibrosis airway epithelia. Cell. 2010;143:911-23.

\title{
S20.2 \\ THE ROLE OF RESPIRATORY VIRUSES AND VIRAL VACCINATION IN EXACERBATIONS
}

\author{
Pedro A. Piedra, M.D. \\ Dept. of Molecular Virology \& Microbiology, Baylor College of Medicine, Houston, TX, USA
}

Respiratory illnesses are a major cause of morbidity and mortality worldwide and respiratory viruses are a major contributor to the burden of respiratory illnesses. In industrialized countries, influenza outbreaks are associated with peaks in cardio-respiratory related hospitalization and deaths among all age groups; respiratory syncytial virus (RSV) is the major cause of bronchiolitis and hospitalization in infants, and rhinovirus is associated with acute exacerbations of chronic respiratory disease such as asthma, chronic obstructive airway disease and cystic fibrosis. Other respiratory viruses such as adenovirus, human metapneumovirus, parainfluenzaviruses, coronaviruses, enteroviruses, human bucavirus, and human respiratory polyomaviruses contribute to the overall societal impact both in disability-adjusted life year (DALY) and financial cost.

Common features related to most of the human respiratory viral pathogens are 1) infection rates are highest in infants and young children; 2) recurrent infections occur throughout life and are milder except in elderly adults and those with co-morbid conditions; 3) illness burden and disease severity is greatest at the extremes of life the young and the elderly; 4) virus-specific serum neu- tralizing antibody (maternally derived, passively administered, or infection-induced) protects against severe lower respiratory tract illness; and 5) co-morbid conditions such as chronic lung disease, heart disease, and immunodeficiency increase the likelihood of severe respiratory disease.

Cystic fibrosis, an inherited chronic lung disease, is unique among the co-morbid conditions with regard to virus-related respiratory tract morbidity. Persons with cystic fibrosis maintain an increased risk for experiencing severe viral respiratory illness throughout life because of their progressive pulmonary inflammation and disease. Infants and young children with $\mathrm{CF}$, like their healthy counterparts, have comparable high rates of infection with respiratory viruses. They differ, however, in their severity and duration of illness. They are significantly more likely compared to healthy infants and young children to develop 1) a lower respiratory tract illness; 2) have a slower illness resolution; 3) an increased risk of hospitalization; 4) a persistent deterioration in lung function; and 5) an increased risk for bacterial colonization with Pseudomonas aeruginosa, Staphylococcus aureus and Haemophilus influenzae (1-3). Increasing 
age normally reduces the risk for severe respiratory disease related to viral infections. Unfortunately, older children with $\mathrm{CF}$ experience frequent bouts of respiratory exacerbations. These events are often related to an acute respiratory viral infection. Respiratory viruses, in particular, rhinovirus, influenza virus, and parainfluenza virus are frequently detected during acute exacerbations in older children resulting in clinical deterioration, intravenous antibiotic treatment, and lung inflammation (47). Improvement in viral diagnostics with use of new molecular diagnostic platforms such as the real-time polymerase chain reaction (rt PCR) can improve our understanding of the role of respiratory viral infections on progressive lung disease experienced in children with CF.

Currently we have limited capabilities in preventing respiratory viruses. The approved products are vaccines against influenza infection and a humanized monoclonal antibody, palivizumab, administered prophylactically for the prevention of RSV-related hospitalization. Palivizum$\mathrm{ab}$ is recommended for a highly select group of infants and young children with medical conditions that increase the risk of severe RSV disease. One such group is children under two years of age with chronic lung disease. CF children may derive benefit from monthly administration of palivizumab during the RSV season, although few studies are available on the benefit of palivizumab in children with $\mathrm{CF}$.

Influenza vaccine is the only approved vaccine for the prevention of a respiratory viral infection. In the United States, the current influenza vaccine recommendation by the Advisory Committee on Immunization Practices (ACIP) of the Centers for Disease Control and Prevention (CDC) is annual influenza vaccination for all persons 6 months of age and older (8). There are currently two major types of influenza vaccine that are FDA approved; the live attenuated influenza vaccine (LAIV) and the inactivated influenza vaccine (TIV). LAIV is approved for persons 2 to $<50$ years old who are in good health and TIV is approved for all persons 6 months of age and older. It is important to note that there are a number of vaccine manufacturers that produce TIV and most are not approved for children less than 4 years of age. Both LAIV and TIV are safe and efficacious with LAIV having superior efficacy compared to TIV in children. The efficacy of LAIV and TIV vary from year to year and in general ranges from 60 to $90 \%$, in part, dependent on how well the vaccine antigens antigenically match the circulating viruses and on the immunocompetence of the host.

There are at least three major vaccination strategies to reduce influenza infection. Vaccination of the individual has a direct benefit by providing immunologic protection. Influenza vaccination coverage in the $\mathrm{CF}$ population is very high in the U.S. and Europe. The CF Foundation, utilizing their patient registry from 2006-2007, reported influenza vaccination coverage of approximately $90 \%$ in children and adults with CF. Underutilization occurred among Hispanics and persons requiring oxygen therapy. A second strategy is maternal immunization. Vaccination of the pregnant women has both a direct benefit to the pregnant women and an indirect benefit to the newborn infant. Infants born to mothers who are vaccinated during pregnancy have a significantly lower risk of influenza infection during their first six months of life. Recall that the influenza vaccine is not approved for use in infants less than 6 months of age. Maternal vaccination against influenza, unfortunately, has been a neglected practice accounting for the lowest vaccination coverage of all target populations. Another strategy is vaccination of select groups who spread the virus efficiently to others such as school-age children, persons who care for or live with persons at high risk, and healthcare workers. This strategy has a direct benefit to the vaccinated person and an indirect benefit to their contacts. Vaccination of school age children has been associated with indirect protection against influenza related complications in unvaccinated household members and the community while vaccination of healthcare workers has been associated with decreased morbidity and mortality in their patients. Surprisingly healthcare workers have suboptimal coverage. Mandatory influenza vaccination programs, although controversial, appears to be the most effective way to significantly improve influenza vaccination coverage among healthcare workers.

References: 1. Van Ewijk BE, Van der Zalm MM, Wolfs TF, et al. Prevalence and impact of respiratory viral infections in young children with $\mathrm{CF}$ : Prospective cohort study. Pediatrics. 2008;122:1171-6.

2. Abman SH, Ogle JW, Butler-Simon N, et al. Role of respiratory syncytial virus in early hospitalizations for respiratory distress of young infants with CF. J Pediatr. 1988;113:826-30.

3. Hiatt PW, Grace SC, Kozinetz CA, et al. Effects of viral lower respiratory tract infection on lung function in infants with CF. Pediatrics. 1999;103:619-26.

4. Wang EEL, Prober CG, Manson B, et al. Association of respiratory viral infections with pulmonary deterioration in patients with cystic fibrosis. N Engl J Med. 1984;311:1653-8.

5. Prober CG. The impact of respiratory viral infections in patients with CF. Clin Rev Allergy. 1991;9:87102.

6. Wat D, Gelder C, Hibbitts $S$, et al. The role of respiratory viruses in cystic fibrosis. J Cyst Fibros. 2008; 7:320-8.

7. Garcia DF, Hiatt PW, Jewell A, et al. Human metapneumovirus and respiratory syncytial virus infections in older children with CF. Pediatr Pulmonol. 2007;42:66-74.

8. Centers for Disease Control and Prevention. Prevention and Control of Influenza with Vaccines. Recommendations of the Advisory Committee on Immunization Practices (ACIP). MMWR Morb Mortal Wkly Rep. 2010;59:989-92. 


\title{
S20.3 \\ KNOWING OUR ENEMY: STUDIES OF THE BACTERIAL POPULATIONS INFECTING CF AIRWAYS
}

Amanda F. Goddard, M.D. ${ }^{2}$, Benjamin Staudinger, M.D. ${ }^{1}$, Scot Dowd ${ }^{3}$, Amruta Datar ${ }^{1}$, Randy Wolcott ${ }^{3}$, Moira L. Aitken, M.D., F.R.C.P. ${ }^{1}$, Corrine Fligner ${ }^{1}$ and Pradeep K. Singh, M.D. ${ }^{1}$

1. Medicine \& Microbiology, Univ. of Washington School of Medicine, Seattle, WA, USA; 2. Dept. of Pediatrics, Univ. of Washington School of Medicine, Seattle, WA, USA; 3. Research \& Testing Laboratory, Lubbock, TX, USA

Recent work suggests that the airways of cystic fibrosis (CF) patients harbor a vast array of bacteria not previously implicated in $\mathrm{CF}$ infections. These findings have fundamentally altered concepts of CF lung disease. For example, culture-independent analysis of bronchoalveolar lavage (BAL) samples indicated that $25 \%$ of children harbored organisms not typically associated with CF. Studies using newer methods are even more striking. Infecting populations consisting of between 206-1329 bacterial taxa per patient were found in upper airway samples analyzed using DNA microarrays. If correct, these findings would require that we fundamentally change established ideas about CF infections.

An important outstanding question, however, is how to interpret these results given the potential that sputum, throat and BAL samples can be contaminated with bacteria from the upper airway. Contamination is a concern for a number of reasons. First, upper airways contain abundant flora, and throat, sputum, and bronchoscopy samples can be mixed with upper airway secretions. Second, culture-independent methods employ 20-35 cycles of PCR amplification, thus even trace amounts of contaminating DNA can produce a robust signal. Third, like the lung, the nasal and pharyngeal epithelium produce mucus that could encase resident bacteria. This complicates efforts to wash contaminating bacteria away, or differentiate them from lung organisms by microscopy. Finally, clinical labs typically disregard organisms known to be oropharyngeal flora to avoid confounding results. Research applications of culture-independent methods bypass these criteria. These factors raise the possibility that non-culture based detection methods could overestimate lung microbial diversity due to upper airway contamination.

The goals of this study were to answer three key questions. First, what bacterial species are present in the lower airways of CF patients with established chronic infections? Identifying the resident bacteria is critical to understanding the mechanisms producing disease. Second, how does oropharyngeal contamination affect culture-independent measurements performed on upper airway specimens? Addressing this question is vital to interpreting culture-independent measurements of infecting population composition at early disease stages. Third, are there consistent differences in the makeup of bacterial populations in distinct anatomic regions of the lung? Finding such differences might explain the regional variation in disease severity often seen in CF lungs. To answer these questions we devised a sterile dissection technique to isolate secretions from the lobar segments of $\mathrm{CF}$ lungs removed at the time of lung transplantation. We measured the composition of infecting populations in these samples using 16S rDNA amplicon pyrosequencing and compared results to paired throat and sputum samples collected hours earlier.

\section{S20.4 \\ NEW THOUGHTS ABOUT NONTUBERCULOUS MYCOBACTERIA INFECTIONS IN CF}

\author{
Kenneth N. Olivier, M.D., M.P.H. \\ Laboratory of Clinical Infectious Diseases, NIAID/NIH, Bethesda, MD, USA
}

Recent epidemiologic studies suggest that cystic fibrosis patients are particularly vulnerable to nontuberculous mycobacterial infections. Prior to 1990, nontuberculous mycobacteria (NTM) had been described in only a handful of CF patients (1). A large cross sectional study that sampled approximately $10 \%$ of the U.S. CF population in the mid 1990s with 3 sputum specimens prospectively collected over a year found an overall prevalence of NTM in $13 \%$ (2). This prevalence appeared to exponentially increase with age ranging from $10 \%$ in children around age 10 to almost $50 \%$ in CF patients over the age of 40. A single site survey of CF patients over the age of 40 found very notable differences in those patients diagnosed early in life (median age of diagnosis around age 2) and later in life (median diagnosis approximately age 27) with the latter group being mostly female and $75 \%$ having NTM in their lower airway (3). Similarly in the general population, CDC surveys of state mycobacteriology lab isolates reported in the 1980s and mid ' 90 s suggested prevalence of 2-8 per 100,000 $(4,5)$. However, more recent studies have noted an increasing prevalence over time with prevalence and relative female predominance increasing markedly by decade over age 50 (6-8). Single site studies of patients presenting with apparent 
idiopathic bronchiectasis associated with NTM have noted a relatively homogenous phenotype of tall, asthenic, post-menopausal women with CFTR abnormalities noted in approximately $30-50 \%(9,10)$. The reasons for this female predominance are not clear but the potential role of female hormones in airway surface defenses has been investigated. Progesterone receptors have been noted at the base of the airway cilia (11). Progesterone has an inhibitory effect on cilia beat frequency that is blocked by estrogen (12). The relative change in balance between progesterone and estrogen that occurs after menopause or perhaps with an asthenic morphotype might alter airway clearance to allow retention of aerosolized environmental organisms like the NTM. Indeed, patients with primary ciliary dyskinesia also appear to have a marked susceptibility to NTM infections with a similar prevalence that appears to increase with age as in CF (13). Investigators have also suggested a potential role of female hormones in macrophage function and intracellular killing of mycobacteria (14).

While Mycobacterium avium complex has been the predominant species isolated in CF patients, M. abscessus seems to be associated with the most morbidity and mortality. Moreover, there appears to be a biphasic interaction with the host whereby it can be intermittently cultured from the respiratory tract of some patients over a prolonged period with no apparent effect on the clinical course followed by a rapid clinical decline associated with an increase in mycobacterial burden (15). It is unclear whether factors accounting for this change in disease severity are host related, represent changes in virulence factors of the organism, or both. Recent studies in France have suggested a change in colony morphology from smooth to rough may correlate with this change in virulence (16). We have not observed a consistent association with clinical course in examination of colony morphology of serial isolates. However, ongoing studies in our lab have focused on identification of possible virulence factors in serial isolates that may be associated with these periods of rapid decline.

A key factor separating tuberculosis from disease caused by NTM has been the apparent mode of acquisition with TB transmission occurring from a human host reservoir and NTM acquisition presumed to be exclusively from an environmental reservoir. Prior epidemiologic studies that have examined the potential for humanto-human transmission of NTM have not found evidence to support this (2). However, a recent outbreak of lethal disease associated with a clonal strain of $M$. massiliense among CF patients in an adult lung transplant center raises significant concerns for the potential of human-to human transmission among a particularly vulnerable population (17).

In summary, CF represents a particularly susceptible population to NTM infections. It is likely this susceptibility relates to altered airway clearance favoring retention of inhaled organisms from contaminated environmental aerosols on the airway surface. The reasons for the age and gender associations are not entirely clear. While these organisms affect a minority of CF patients, disease associated with the $M$. abscessus group can be particularly devastating. The recently described outbreak of $M$. massiliense among CF patients awaiting transplant might warrant similar infection control considerations for some species as are recommended for other virulent organisms such as Burkholderia cepacia. Identification of specific inducible virulence factors that might allow a more refined approach to infection control and better targeting of aggressive treatment regimens is of key importance.

References: 1. Olivier KN, Yankaskas JR, Knowles MR. Nontuberculous mycobacterial pulmonary disease in CF. Semin Respir Infect. 1996;11:272-84.

2. Olivier KN, Weber DJ, Wallace RJ Jr, et al. Nontuberculous mycobacteria. I: Multicenter prevalence study in cystic fibrosis. Am J Respir Crit Care Med. 2003;167:828-34.

3. Rodman DM, Polis JM, Heltshe SL, et al. Late diagnosis defines a unique population of long-term survivors of CF. Am J Respir Crit Care Med. 2005;171:621-6.

4. O'Brien RJ, Geiter LJ, Snider DE Jr. The epidemiology of nontuberculous mycobacterial diseases in the U.S. Results from a national survey. Am Rev Respir Dis. 1987;135:1007-14.

5. Butler WR, Crawford JT. Nontuberculous mycobacteria reported to the public health laboratory information system by state public health laboratories U.S., 1993-1996. Atlanta, Ga: Centers for Disease Control and Prevention; 1999.

6. Prevots DR, Shaw PA, Strickland D, et al. Nontuberculous mycobacterial lung disease prevalence at four integrated health care delivery systems. Am J Respir Crit Care Med. 2010;182:970-6.

7. Marras TK, Chedore P, Ying AM, et al. Isolation prevalence of pulmonary non-tuberculous mycobacteria in Ontario, 1997-2003. Thorax. 2007;62:661-6.

8. Winthrop KL, McNelley E, Kendall B, et al. Pulmonary nontuberculous mycobacterial disease prevalence and clinical features: An emerging public health disease. Am J Respir Crit Care Med 2010;182:977-82.

9. Kim RD, Greenberg DE, Ehrmantraut ME, et al. Pulmonary nontuberculous mycobacterial disease: Prospective study of a distinct preexisting syndrome. Am J Respir Crit Care Med. 2008;178:1066-74.

10. Ziedalski TM, Kao PN, Henig NR, et al. Prospective analysis of cystic fibrosis transmembrane regulator mutations in adults with bronchiectasis or pulmonary nontuberculous mycobacterial infection. Chest. 2006;130:995-1002.

11. Jia S, Zhang X, He DZ, et al. Expression and function of a novel variant of estrogen receptor-er- $\{$ alpha $\} 36$ in mouse airway. Am J Respir Cell Mol Biol. 2011 Jun 3. [Epub ahead of print]. 
12. Jain R, Ray J, Brody SL. Sex hormone-dependent activation of the progesterone receptor in airway epithelial cells inhibits cilia beat frequency. Am J Respir Crit Care Med. 2011;183:A1226.

13. Noone PG, Leigh MW, Sannuti A, et al. Primary ciliary dyskinesia: Diagnostic and phenotypic features. Am J Respir Crit Care Med. 2004;169:459-67.

14. Tsuyuguchi K, Suzuki K, Matsumoto H, et al. Effect of oestrogen on Mycobacterium avium complex pulmonary infection in mice. Clin Exp Immunol 2001;123:428-34.
15. Cullen AR, Cannon CL, Mark EJ, et al. Mycobacterium abscessus infection in CF. Colonization or infection? Am J Respir Crit Care Med. 2000;161:641-5.

16. Roux AL, Ray A, Pawlik A, et al. Overexpression of proinflammatory tlr-2-signalling lipoproteins in hypervirulent mycobacterial variants. Cell Microbiol. 2011;13:692-704.

17. Aitken ML, Goss CH, Tonelli MR, et al. Mycobacterium abscessus subspecies massiliense in a respiratory outbreak in a lung transplant and CF center. Am J Respir Crit Care Med. 2011 (in press). 